
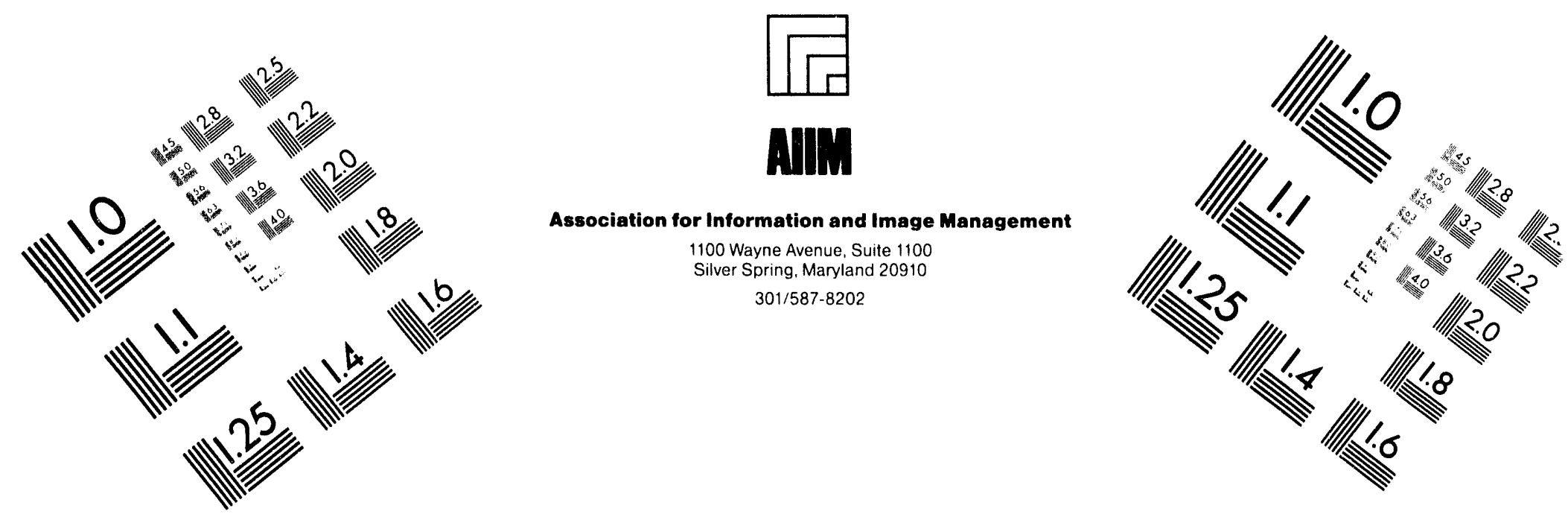

\title{
Centimeter
}

10 Inches
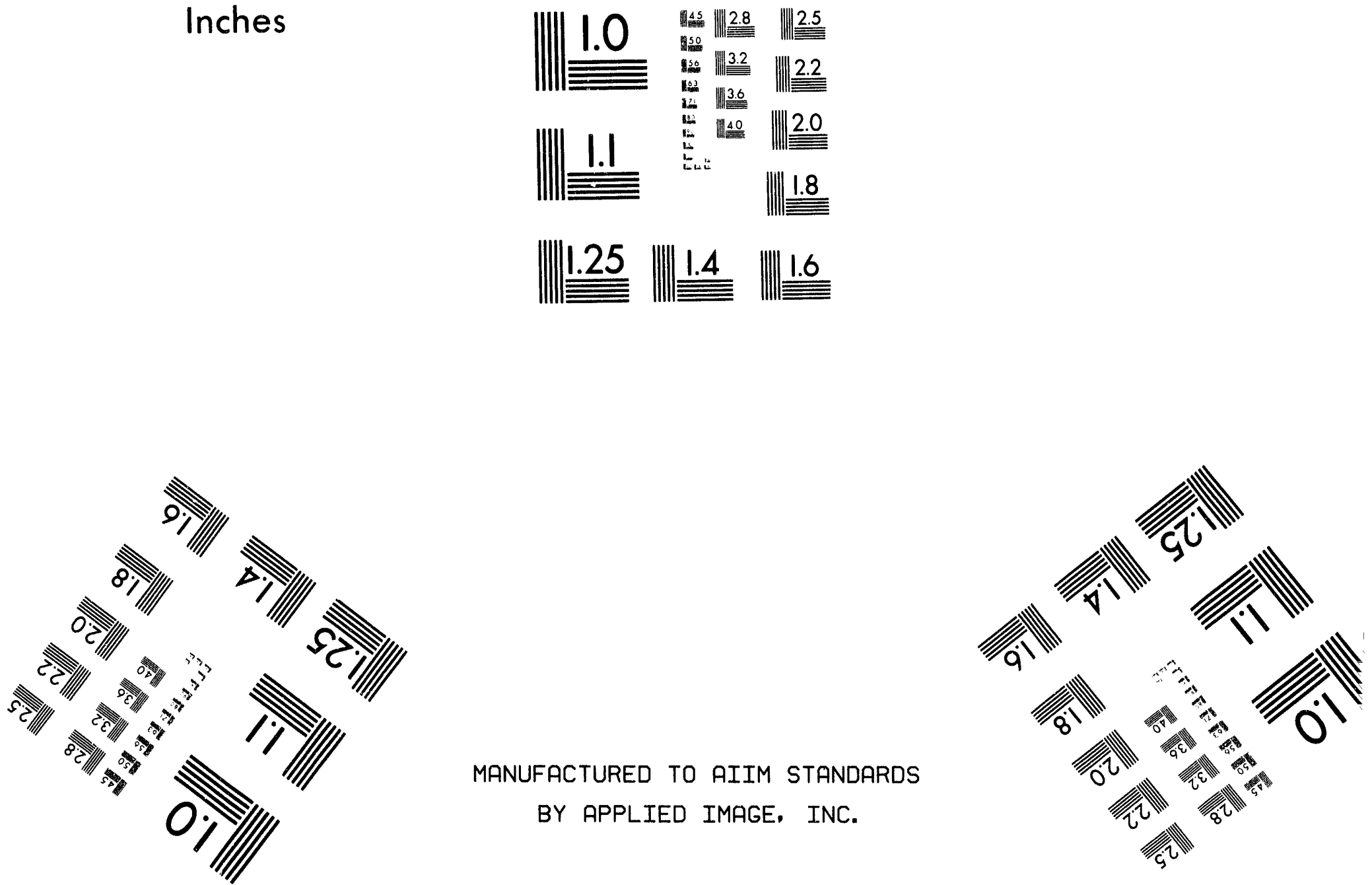

BY APPLIED IMAGE, INC. 

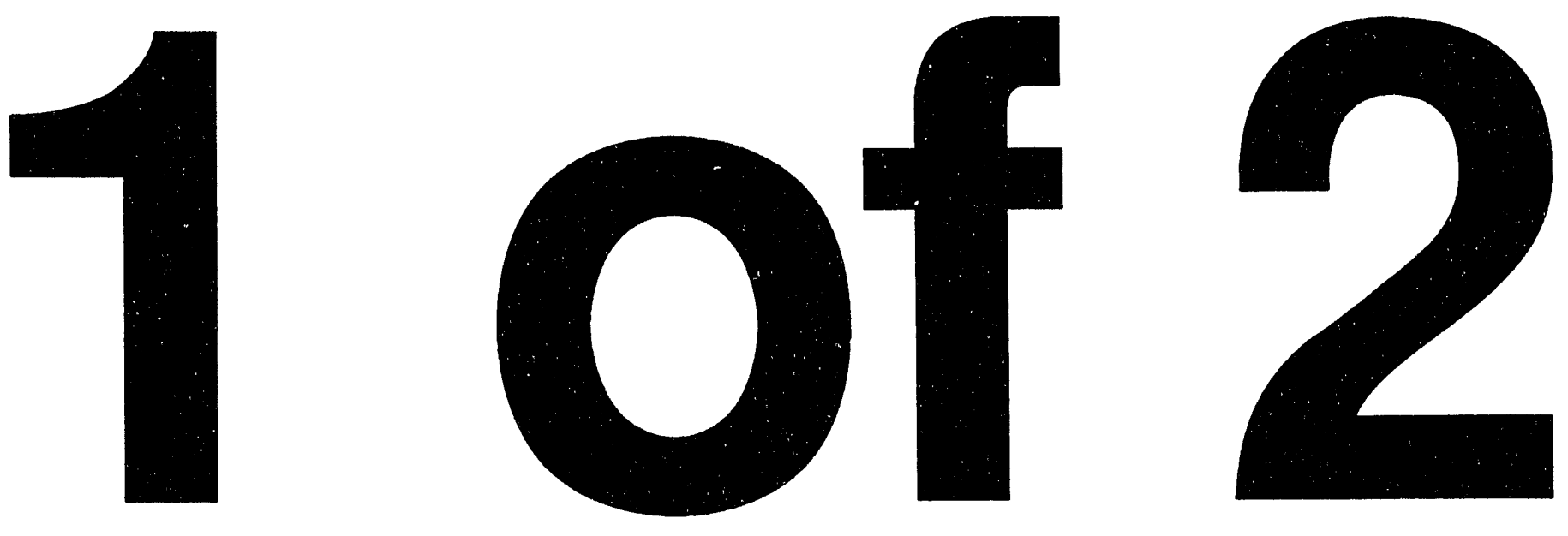


\section{Tank Farm Surveillance and Waste Status Summary Report for May 1994}

B. M. Hanlon

August 1994

Prepared for the U.S. Department of Energy Office of Environmental Restoration and Waste Management

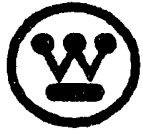




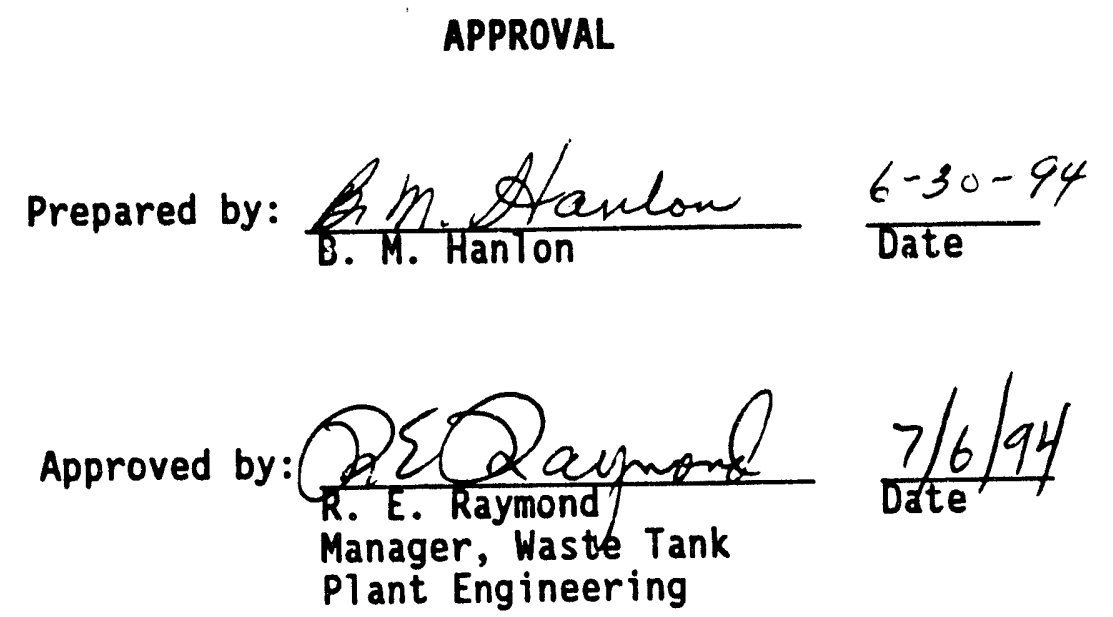




\section{RELEASE AUTHORIZATION}

\section{Document Number: WHC-EP-0182-74}

Document Title: TANK FARMS SURVEILLANCE AND WASTE STATUS SUMMARY REPORT FOR MAY 1994

Release Date: $\quad 8 / 18 / 94$

This document was reviewed following the procedures described in WHC-CM-3-4 and is:

APPROVED FOR PUBLIC RELEASE

WHC Information Release Administration Specialist:

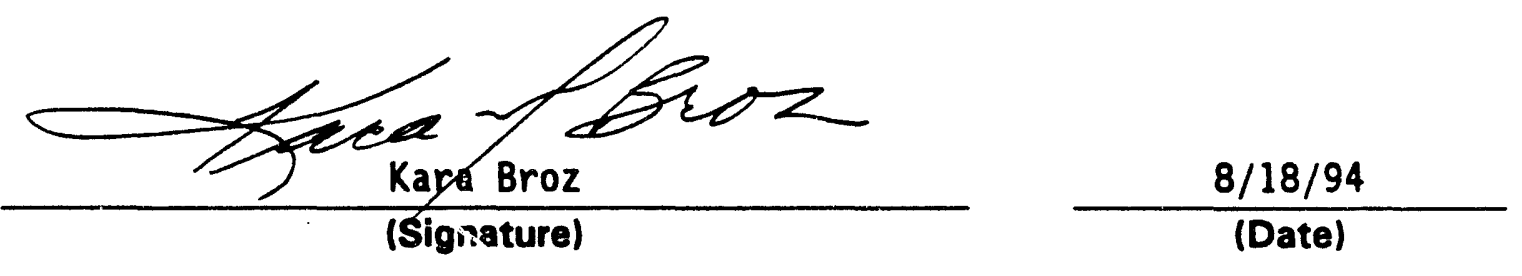




\title{
TANK FARM SURVEILLANCE AND WASTE STATUS
}

SUMMARY REPORT FOR MAY 1994

\author{
B. M. Hanlon
}

\section{ABSTRACT}

This report is the official inventory for radioactive waste stored in underground tanks in the 200 Areas at the Hanford site. Data that depici the status of stored radioactive waste and tank vessel integrity are contained within the report. This report provides data on each of the existing 177 large underground waste storage tanks and 49 smaller catch tanks and special surveillance facilities, and supplemental information regarding tank surveillance anomalies and ongoing investigations. This report is intended to meet the requirement of U. S. Department of Energy-Richland Operations Office Order 58. 0.2A, Chapter I, Section 3.e. (3) (DOE-RL, 1990, Radioactive Waste Management, U. S. Department of Energy-Richland Operation Office, Richland, Washington) requiring the reporting of waste inventories and space utilization for Hanford Tank Farm Tanks. 
WHC-EP-0182-74

This page intentionally left blank. 
WHC-EP-0182-74

CONTENTS

SUMMARY . . .................... 1

I. WASTE TANK STATUS ................... 1

II. WASTE TANK INVESTIGATIONS . . . . . . . .... 2

III. SURVEILLANCE AND WASTE TANK STATUS HIGHLIGHTS . . . . . . . . . . 7

Appendixes:

A. WASTE TANK SURVEILlANCE MONITORING TABLES ........... A-1

Tables:

1 Watch List Tanks . . . . . . . . . . . . . . . A-3

2 Tanks Containing $>1000$ Gram Mole of Ferrocyanide . . . . A-5

3 Tanks Hith Potential for Hydrogen or Flammable Gas

Accumulation above the Flammability Limit . . . . . . A A-7

4 Single-Shell Tanks Containing Concentrations of Organic Salts . A-8

5 Single-Shell Tanks With High Heat Loads $(>40,000 \mathrm{Btu} / \mathrm{h}) \ldots$. . A-10

6 Non-Watch List Low Heat Load Tanks $(<40,000 \mathrm{Btu} / \mathrm{h}) \ldots \ldots-12$

7 Single-Shell Tanks Monitoring Compliance Status . . . . . . A-15

8 Double-Shell Tanks Monitoring Compliance Status . . . . . . A-20

9 Automatic Food Instrument Corporation (FIC) Gauges

Out of Service ................ A-22

Figures:

1 Discrepancy Report Status ............... A-23

2 Discrepancy Report Cumulative Total ............ A-24

3 Discrepancy Report Status by Age . . . . . . . . . . . A-25

B. DOUBLE-SHELL TANK WASTE TYPE AND SPACE ALlOCATION . . . . . . . B-1

Tables:

1 Double-Shell Tank Waste Type and Space Allocation . . . . . B-3

2 Double-Shell Tank Waste Inventory .......... B-10

Fiqures:

1 Total Double-Shell Tank Inventory and Changes. . . . . . B-4

2 Usable Tank Space Inventory and Changes ........... B-5

3 Contributions to Priority Space ........... . . B-6

4 Comparison of Facility Generations to Management Limit . . . . B-7

5 Comparison of Monthly Average Waste Generation to

Management Limit by Facility . . . . . . . . . . B-8

6 Overall Waste Flow - Hanford Tank Waste Disposal . . . . . B-9

c. TANK AND EQUIPMENT CODE AND STATUS DEFINITIONS ......... c-1

1 Tank and Equipment Code/Status Definitions.......... C-3

D. TANK FARM CONFIGURATION, STATUS AND FACILITY CHARTS . . . . . . D-1

Figures:

1 High-Level Waste Tank Configuration ......... D-3

2 Double-Shell Tank Instrumentation Configuration . . . . D-4

3 Single-Shell Tank Instrumentation Configuration ...... D-5

4 Double-Shell Tank Status ............. . . D-7/8

5200 E Single-Shell Tank Status . . . . . . . . . . . . . D-9/10

$6200 \mathrm{~W}$ Single-Shell Tank Status .............. . D-11/12

7 Hanford Tank Farms Facilities Chart: 200-East Area . . . . . D D-13/14

8 Hanford Tank Farms Facilities Chart: 200-West Area . . . . . . D-15/16 
WHC-EP-0182-74

This page intentionally left blank 
E. MONTHLY SUMMARY . . . . . . . . . . . . . . . . E-1 Tables:

1 Monthly Summary . . . . . . . . . . . . . E-3

2 Tank Use Summary . . . . . . . . . . . . . . E-4

3 Inventory Summary by Tank Farm . . . . . . . . . . E-5

4 Inventory and Status by Tank - Double-Shell Tanks . . . . . . E-6

5 Inventory and Status by Tank - Single-Shell Tanks ....... E-9

F. PERfORMANCE SUMMARY .................. F-1 Table:

1 Performance Summary ................ . . . . . .

G. PUMPING RECORD/LIQUID STATUS AND PUMPABLE LIQUID REMAINING IN TANKS . . G-1 Tables:

1 Pumping Record . . . . . . . . . . . . . . . G-3

2 Liquid Status and Pumpable Liquid Remaining in Tanks ......G-4

H. CATCH TANKS AND SPECIAL SURVEILLANCE FACILITIES . . . . . . . . H-1 Tables:

1 East and West Area Catch Tanks and Special

Surveillance Facilities (Active) . . . . . . . . . H-3

2 East Area Catch Tanks and Special Surveillance

Facilities (Inactive) .............. . H-4

3 West Area Catch Tanks and Special Surveillance

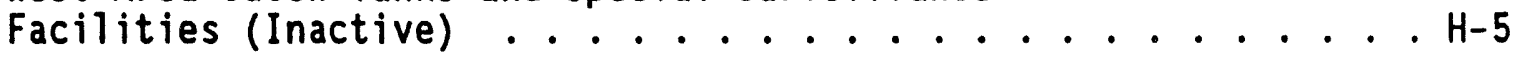

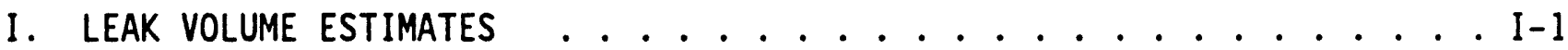

Table:

1 Single-Shell Tank Leak Volume Estimates . . . . . . . . . I-3

J. INTERIM STABILIZATION STATUS ..................... . . . . Table:

1 Single-Shell Tanks Interim Stabilization Status ....... J-3 Figure:

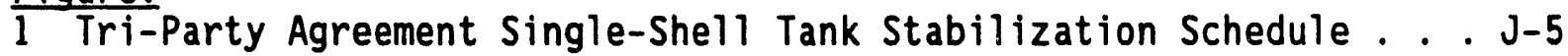

K. TANK FARM OPERATIONS SAMPLING SCHEDULE STATUS ........... . K-1 Table:

1 Tank Farm Operations Sampling Schedule Status ......... K-3 


\begin{tabular}{|l|c|c|}
\hline \multicolumn{3}{|c|}{ METRIC CONVERSION CHART } \\
\hline 1 inch & $=$ & 2.54 centimeters \\
\hline 1 foot & $=$ & 30.48 centimeters \\
\hline 1 gallon & $=$ & 3.80 liters \\
\hline 1 ton & $=$ & 0.90 metric tons \\
\hline \multicolumn{2}{|c}{$\mathrm{F}=\left(\frac{9}{5}{ }^{\circ} \mathrm{C}\right)+32$} \\
\hline \\
$\begin{array}{r}1 \mathrm{Btu} / \mathrm{h}=2.930711 \text { E-01 watts } \\
(\text { International Table })\end{array}$ \\
\hline
\end{tabular}




\section{TANK FARM SURVEILLANCE AND WASTE STATUS SUMMARY REPORT FOR MAY 1994 SUMMARY}

Note: Changes from the previous month are in bold print.

\section{WASTE TANK STATUS}

\begin{tabular}{|c|c|c|}
\hline Categcry & Quantity & Date of Last Change \\
\hline In-Service Tanks ${ }^{c}$ & 28 double-shell & $10 / 86$ \\
\hline Out-of-Service Tanks & 149 single-shell & $07 / 88$ \\
\hline Assumed Leaker Tanks ${ }^{f}$ & 67 single-shell & $7 / 93$ \\
\hline Sound Tanks & $\begin{array}{l}28 \text { double-shell } \\
82 \text { single-shell }\end{array}$ & $\begin{array}{l}1986 \\
7 / 93 \\
\end{array}$ \\
\hline Interim Stabilized Tanks ${ }^{b, d}$ & 106 single-she11 & $04 / 93$ \\
\hline Not Interim Stabilized ${ }^{\dagger}$ & 43 single-shell & $04 / 93$ \\
\hline Intrusion Prevention Completed & 98 single-shell & $09 / 91$ \\
\hline $\begin{array}{l}\text { Watch List Tanks } \\
\text { Total }\end{array}$ & $\begin{array}{l}50 \text { single-shel1 } \\
6 \text { double-shel1 } \\
56 \text { tanks }\end{array}$ & $\begin{array}{l}5 / 94^{h} \\
6 / 93\end{array}$ \\
\hline
\end{tabular}

- Although all 149 single-shell tanks were removed from service (i.e., no longer authorized to receive waste) as of November 21, 1980, the category of "Out-of-service" was not established until July 1988.

b of the 106 tanks classified as interim stabilized, 59 are listed as assumed leakers. The total of 106 interim stabilized tanks includes six tanks that do not meet current establ ished supernatant and interstitial liquid stabilization criteria: B-104, B-110, B-111, T-102, T-112, and U-110. (These six tanks did meet the criteria in existence when they were declared interim atabilized). B-110, B-111, and U-110 are assumed leakers but surveillance data do not show an indication of a continuing leak.

c six double-shell tanks listed as "in service" are currently included on the Hydrogen Watch List and are thus prohibited from receiving waste in accordance with usafety Measures for Waste Tanks at Hanford Nuclear Reservation," Section 3137 of the Mational Defense Authorization Act for Fiscal Year 1991. Novenber 5, 1990, Public Law 101-510.

d of the 50 single-shell tanks on Watch Lists, 19 have been Interim Stabilized.

- of the 50 single-shell tanks on Watch Lists, 28 have completed Intrusion Prevention (this category replaced Interin Isolation). See Appendix C, Tank and Equipment Codes and Status Definitions, for "Intrusion Prevention" definition.

f Eight of the tanks are both assuned leakers and not Interia stabilized. See Appendix I, Leak Volume Estimates, for more details. Tank sx-102 was declared an aseuned leaker in May, and reclassified as sound in July, 1993. See maste Tenk Investigations" section of the July 1993 report for more details.

- See Tables A-1 through A-5 for more information on watch List Tenks. Ten tanks (A-101, S-102, s-111, sX-103, SX-106, TX-118, TY-104, U-103, U-105, and U-107) ore currently on wore than one Watch List.

$h$ Dates for the Watch List tanks are "officially added to the Watch List" dates. See Table A-1, Watch List Tanks, for further information. 


\section{WASTE TANK INVESTIGATIONS}

This section includes all single-shell tanks or catch tanks which are showing surface level or interstitial liquid level (ILL) decreases, or druwell/ lateral radiation level increases in excess of established criteria.

There were no tanks under investigation for ILL decreases or drywe $11 / 1$ ateral radiation level increases which exceeded the criteria in May 1994.

A. Assumed Leakers or Assumed Re-leakers: (See Appendix C for definition of "Re-leaker")

This section includes all single- or double-shell tanks or catch tanks for which an off-normal or unusual occurrence report has been issued for assumed leaks or re-leaks. Tanks/catch tanks will remain on this 1 ist until either a) completion of Interim Stabilization, or b) the updated occurrence report indicates that the tank/catch tank is not an assumed leaker.

Tank 241-BX-111. This tank was declared an assumed re-leaker on April 30, 1993. Pumping of the tank commenced on October 22, 1993. Pumping was completed as of April 30, 1994. Flushing activities were done in May, to confirm the pumping completion. $3.3 \mathrm{Kgals}$ were pumped, $110.4 \mathrm{Kgal}$ pumped as of May 31, 1994. (See Table E-5 footnotes for further information).

Tank 241-T-111. The surface level has shown a steady decrease since the automatic FIC was repaired in August 1993. The surface level measurement after the FIC repair was 161.70 inches and continued to decrease to 161.10 inches by January 31, 1994. This is a 1.00-in decrease from the reference baseline of 162.10 inches. Off-Normal Occurrence Report RL-WHC-TANKFARM-19940009 was issued on February 24, 1994. This tank was declared an assumed releaker on February 25, 1994.

This tank had previously been Partial Interim Stabilized. Tank T-111 was added to the Organics Watch List un February 28, 1994. In-tank photos were taken April 13, 1994. Review of these photos resulted in some changes in the tank's inventory. (See Table E-5 footnotes for April 1994).

Pumping began May 17, 1994, completing a TPA milestone for the start of emergency pumping before May 30, 1994. Total pumped was $3.1 \mathrm{Kgal}$ as of May 30, 1994. (See Table E-5 footnotes for further information).

\section{B. Tanks with increases indicating possible intrusions:}

This section includes all single-shell tanks for which the surveillance data show that the surface level or ILL has met or exceeded the increase criteria, or are still being investigated.

244-AR Tanks ano SUmps: Currently, all ventilation systems at 244-AR are shut down. Based on the weight factors for the sumps and tanks, Tank 001 contains 2300 gallons, Tank 002 contains 8100 gallons (some unknown amount of sludge), Tank 003 contains 2100 gallons, and Tank 004 contains 500 gallons. Sump 003 increased to approximately 250 gallons of intrusion water (rain) over the past three months because of rainfali. Currentiy, Sump 003 contains approximately 450 gallons of water. 
Tank 241-B-202. A steady increase in the surface level measurement has been observed since Dacember 1984. The manual tape pencil plummet is contacting liquid. When th- quarterly reading was obtained on October 6, 1992, the level was recorded as 144.75 inches, thus exceeding the 2.00-inch increase criteria from the established baseline of 142.50 inches. The surface level measurement was rechecked on October 9, 1992, (145.50 inches), verifying the increase and that the criteria had been exceeded. Occurrence Report RL-WHC-TANKFARM-19930024 was issued February 13, 1993. The surface level remained stable at 145.75 ( \pm 0.25 inches) during May, 1994. The monitoring frequency has been increased from quarterly to daily. This tank is Sound, Interim Stabilized, and Intrusion Prevention completed.

Resolution status: A photo package was initiated on May 11, 1993, to investigate the possibility of an intrusion. Review of previous photos was inconclusive. New photos are required to determine the actual supernatant increase, if any. A temporary baseline was established at 145.25 inches, until the new photos are available.

Tank 241-BX-101. On September 2, 1993, the surface level increased from 10.00 to 12.00 inches. The surface level was 11.75 inches on May 31, 1994. In-tank photographs show the manual tape donut plummet contacting liquid in shallow pool. This tank is an Assumed Leaker, Interim Stabilized, and Intrusion Prevention completed.

Resolution Status: Comparison of October 1986 photos with November 1988 photos shows evidence of an ongoing intrusion. A work package was initiated October 14, 1993, to obtain in-tank photographs which will be used to inspect the area under the plummet and investigate the possible intrusion. At current manpower levels, photos in this tank should be available by October 1994.

Iank 241-BX-103. This tank has shown an erratic increase in surface level measurements since January 6,1986 . On January 18, 1993, the surface level measurement in 103-BX exceeded the 0.50 -inch increase criteria from the reference baseline of 19.50 inches, and was verified on January 20,1993 . Discrepancy Report S\&DA 93-522 was issued January 21, 1993. Occurrence Report RL-WHC-TANKFARM-1993-0036 was issued March 25, 1993. The surface level measurement is currently 20.50 inches. The FIC plummet is contacting liquid as indicated by in-tank photographs taken October 31, 1986. This tank is Sound, Interim Stabilized, and Intrusion Prevention completed.

Resolution status: The current level is greater than that prior to stabilization in November 1983. The tank was previously determined to have experienced an intrusion from 1977 to March 1983 (prior to stabilization). Subsequent isolation was expected to halt the intrusion, however, the intrusion is apparently ongoing. A work package was initiated on May 11 , 1993, to obtain in-tank photos. The photos will be used to assess the current stabilization status of the tank. A visual survey of the area was performed to determine possible paths for precipitation to enter the tank. The weather covering on the pits and risers was found in place and undamaged. The existing grade is level and revealed no obvious draining problems. Design/isolation drawing review revealed that nozzles, floor drains and some transfer lines entering the heel pit have been left open. Neich hring tank BX-101 was investigated in 1987 and recommendations were provideu is halt an ongoing intrusion. Similar measures may halt the BX-103 intrusion. In-tank 
photos will provide verification of the intrusion. Determination of the need to provide additional isolation measures will follow. At current manpower levels, photos in this tank should be available by November 1994.

Tank 241-BY-105. Although the surface level and ILL are within the criteria limits, the data indicates unusual behavior trends that merit continued observation. The monitoring frequency has been increased from quarterly to weekly. This tank is on the ferrocyanide Watch List, an Assumed Leaker, and not yet Interim Stabilized.

Resolution Status: The surface level and ILL are displaying behavior simi?ar to $T X-113$ and $T X-115$. The ILL is showing an increase, while the surface level measurement is showing a decrease. This phenomena could be due to either solids dissolution or formation of a depression in the solids beneath the plummet in conjunction with an intrusion. Review of previous photos indicates the liquid volume is increasing, although it cannot be verified that the solids level is decreasing. A photo package was initiated to investigate the possibility of intrusion, or solids dissolution. At current manpower levels, photos in this tank should be available by April 1995.

Tank 241-S-102. The FIC was out of service from November 16, 1993, to January 14, 1994. The surface level reading after the FIC was repaired on January 14 was 207.00 inches. This level exceeded the 3.00 -inch increase criteria from the reference baseline of 202.30 inches. Reference Discrepancy Report S\&DA-904-672. This tank has a history of erratic increases/decreases. In-tank photographs taken March 18, 1988, show the FIC plummet contacting an uneven, dry surface. A verification of the reference elevation was completed February 27, 1994. The FIC counter was found to be out of calibration by +2.60 inches. On May 27, 1994, the baseline was adjusted to 205.80 inches. The surface level reading on May 31, 1994, was 206.00 inches. The LOW ILL was within the 0.10-foot tolerance when last scanned on May 23, 1994. This tank will be removed from the report unless further activities warrant its inclusion.

Resolution status: Photos taken in March 1988, show the surface consists of very uneven solids with scattered liquid pools. Photos taken in 1983 compared with the 1988 photos indicate the waste is shifting, possibly causing a change in surface level under the plummet. Reevaluation of the ILL using the "new method" shows an increasing trend of about 0.4 inches per year. Occurrence Report 77-191 attributed previous intrusions into the tank to drainage from the $S A$ and $S B$ valve pits. Because neither the tank nor the pits are interim isolated, the intrusion is likely ongoing. A photo package was initiated on May 17, 1993, to confirm the ongoing intrusion. This tank is on the hydrogen and organic salts Watch List. A safety basis has been established for performing in-tank photography in tanks of this nature. In-tank photos may be performed provided continuous sampling is done through a different tank riser. At current manpower levels, photos in this tank will be available by December 1996.

Tank 241-TX-111. Although the surface level and ILL measurements do not exceed the criteria, the data indicates unusual behavior trends that merit continued observation. This tank is Sound, Interim Stabilized, and Intrusion Prevention completed. 
Resolution Status: The ILL trend was re-analyzed for this tank using the new "count rate" method. A steady, significant increase of 1.32 inch/year is evident. Surface level data is showing a decrease. Photos show a dry surface. The surface level decrease is expected to be caused by crumbling of the waste beneath the plummet. Overall, the waste level behavior of the tank is similar to that of $T X-113$ and 115. A photo package was initiated on October 13, 1993, to investigate the possibility of an intrusion. At current manpower levels, photos in this tank will be available by November 1995.

Tank 241-TX-113. Although the surface level and ILL measurements do not exceed the criteria, the data indicates unusual behavior trends that merit continued observation. This tank is an Assumed Leaker, Interim Stabilized, and Intrusion Prevention completed.

Resolution status: The technical evaluation of the alert condition in this tank was completed April 14, 1993. The results were inconclusive, with recommendation to accelerate the October 1997 waste characterization of the tank. This characterization is expected to confirm that solids are dissolving, causing an increase in ILL. Acceleration of waste

characterization is not possible. Watch List tanks have first priority for core sampling. A photo package has been written. At current manpower levels, photos in this tank will be available by December 1995. The LOW is scanned weekly.

Tank 241-TX-115. Although the surface level and ILL measurements do not exceed the criteria, the data indicates unusual behavior trends that merit continued observation. This tank is an Assumed Leaker, Interim Stabilized, and Intrusion Prevention completed.

Resolution status: The technical evaluation of the alert condition in this tank was completed April 14, 1993. The results were inconclusive, with recommendation to accelerate the October 1997 waste characterization. Waste characterization is expected to confirm that solids are dissolving, causing an increase in ILL. Acceleration of waste characterization is not possible for non-Watch List tanks. The 1981 photos show evidence of rain intrusion through a central pump pit riser. However, it cannot be concluded from the 1988 photos that the intrusion is ongoing. A photo package has been written. At current manpower levels, photos in this tank will be available by February 1996. The LOW is scanned weekly. This tank will be included in the waste characterization with TX-113.

Tank 241-TY-102. Discrepancy Report S\&DA-92-489 was issued November 9, 1992, when the surface level measurement exceeded the 0.50 -inch increase criteria from the established baseline of 31.40 inches. The tank has a history of intrusions and icicle-shaped mineral buildup on the FIC plummet. The FIC plummet is contacting a shallow pool of liquid. The surface level measurement on Apri1 1, 1993, was 31.90 inches and at the increase criteria. The increase criteria of 0.50 inch, considered to be an extremely tight tolerance for this tank, was revised to 1.00 inch, which is more in line with other tanks that exhibit similar erratic surface level behavior. The surface level measurement remained stable during May and was 31.80 inches on May 31,1994 . This tank is Sound, Interim Stabilized, and Intrusion Prevention completed.

Resolution status: This tank is experiencing an ongoing intrusion as is evident by comparison of November 1984 photos with July 1987 photos and an 
increasing trend in surface level data. A visual survey was conducted to determine possible paths for precipitation to enter the tank. The grade around the tank is level and weather covering is on the pits and intact. The photo package, initiated May 17, 1993, will be required to assess the current stabilization status of the tank, and to assist in determining the possible paths of intrusion. Ongoing design/isolation drawing review revealed that nozzles, floor drains and some transfer lines entering the pump pit have been left open. Photo verification of the intrusion will determine the need to provide additional isolation measures. At current manpower levels, photos in this tank will be available by March 1996.

\section{Catch Tanks:}

241-AX-152 Diverter Station. The surface level measurement exceeded the 30.00-inch action limit and the 48.00-inch maximum operating limit, but did not exceed the Operating Specifications Document (OSD) 1 imit of 77.00 inches. The tank was pumped on May 21, 1994. The surface level dropped from 71.75 inches to 14.75 inches after being pumped. The level was 15.00 inches on May 31, 1994.

Resolution status: Alternative methods to supply water for transfer are underway due to a water line leak in the A-Farm complex. This tank will not appear in future reports unless further activities warrant its inclusion.

241-ER-311 Catch Tank. This catch tank shows increases from precipitation and runoff. The tank currently exceeds the active tank limit of $45 \%$ volume ( 8000 gallons). This tank may contain up to $80 \%$ of volume capacity, $(14,100$ gallons, 80.00 inches) during inactive periods.

Resolution Status: A procedure is being drafted to pump this tank. The procedure is expected to be completed in September 1994.

241-E/W-151 Vent Station Catch Tank. The zip cord surface level reading exceeds the maximum operating limit of 36.00 inches. The manual tape was out. of service from July 7, 1992, to December 16, 1992, when a temporary zip cord was installed. A surface level reading of 68.00 inches was obtained, exceeding the active tank limit of $50 \%$ volume of 40 inches ( 400 gallons). Discrepancy Report S\&DA-92-511 was issued December 24, 1992. Transfers are not permitted until the tank is pumped and the level is within limits. A new calibrated zip cord was installed December 16, 1993, and the surface level reading went from 71.00 to 59.00 inches. Discrepancy Report 93-655 was issued December 17, 1993. The zip cord was replaced with a new manual tape on December 23, 1993. The level reading was 60.50 inches. The surface level reading was 65.55 inches on May $31,1994$.

Resolution status: The catch tank was sampled on December 23, 1993. The waste is to be transferred using existing cross-site lines. The required procedure is in approval stages; other documentation has been compleced. Transferring with this method could be ready by late June.

241-UX-302-A Catch Tank. The surface level measurement exceeds the maximum operating limit of 50.00 inches. Discrepancy report S\&DA-92-465 was issued May 12, 1992. The current surface level reading is 68.60 inches, which exceeds the $50 \%$ volume of 54 inches ( 8840 gallons). The FIC plummet is contacting liquid. 
Resolution status: Work packages for the transfer of waste from UX-302-A and the repair of necessary instrumentation are being prepared, and the transfer of waste is being scheduled as a prestart item for the cross-site transfer. A work package has been prepared to sample/pump this catch tank. Transfer will begin after completing Vent Station transfer. Procedures are being prepared.

\section{SURVEILLANCE AND WASTE TANK STATUS HIGHLIGHTS}

\section{Single-Shell Tanks Saltwell Jet Pumping}

Tank 241-BX-110 - Saltwell jet pumping began December 4, 1993, to pump contents of this tank into 244-BX Double Container Receiver Tank (DCRT). Saltwell pumping was shut down on December 9, 1993, for repair of system air leaks. After repair of the leaks, it was determined other problems existed, resulting in a work order to modify the system to run the pump manually. It is uncertain whether any further waste can actually be pumped. A total of 4.0 $\mathrm{Kgal}$ has been pumped from BX-110.

Tank 241-BX-111 - Saltwel1 jet pumping began on October 22, 1993, to pump tank BX-111 into 244-BX Double Container Receiver Tank. Pumping was considered complete as of April 29, meeting a DOE milestone. In-tank photos were taken on May 19, 1994. After review of photos, the pumping was restarted to remove additional pumpable liquid. $3.3 \mathrm{Kgal}$ were pumped, making a total of $110.4 \mathrm{Kgal}$ pumped as of May 31, 1994. (See Table E-5 footnotes for further information).

Tank 241-BY-102 - Saltwell jet pumping resumed May 30, 1994, after being suspended in 1992 due to an Unreviewed Safety Question (USQ) and other issues. (See item $\$ 5$ below). Restart of the pumping completed a TPA milestone. $2.3 \mathrm{Kgal}$ were pumped from this tank in May, with a total of 125.6 Kgal pumped by May 31, 1994. (See Table E-5 footnotes for further information).

Tank 241-BY-109 - Saltwell jet pumping resumed May 31, 1994, after being suspended in 1992 due to an Unreviewed Safety question (USQ) and other issues. (See item $\# 5$ below). Restart of the pumping completed a TPA milestone. $0.1 \mathrm{Kgal}$ were pumped, making a total of $93.6 \mathrm{Kgal}$ pumped by May 31, 1994.

Tank-241-T-111 - Saltwell jet pumping began on May 17, 1994, completing a TPA milestone for emergency pumping. 3.1 Kgal was pumped by May 31, 1994. In-tank photos were taken May 19, 1994. (See Table E-5 footnotes for further information).

\section{Tank Waste Remediation System Safety Initiatives}

The U. S. Secretary of Energy has directed that six safety initiatives be implemented in the Tank Waste Remediation System Program to accelerate the mitigation/resolution of the higher priority waste tank safety issues at the Hanford Site. Forty-two milestones were established for accomplishing the initiatives.

Safety Initiative "Close Tank C-103 Unreviewed Safety Question" was closed in May 1994. 


\section{Tank Waste Analysis Plan}

The 1995 Fiscal Year Tank Waste Remediation System - Tank Waste Analysis Plan was submitted to DOE-RL for transmission to the U. S. Environmental Protection Agency and the Washington State Department of Ecology. The plan is

a guidance document that directs the sampling and analysis of core, auger, and grab samples through Tank Characterization Plans. Transmission of this pian completes a TPA milestone due May 31, 1994.

\section{Tank Farms Stand Down (Administrative Hold)}

On August 12, 1993, non-essential work activities in the tank farms were put on administrative hold by WHC senior management until operators, supervisors and managers are retrained to perform their duties in a safe and accountable manner. Minimal essential activities required for safety, monitoring and compliance, including the daily "pump-bumping" of the SY-101 mixer pump, will continue. The administrative hold has impacted several programmatic goals for Tank Waste Remediation System (TWRS). A letter from WHC senior management has been sutmitted to DOE-RL addressing these programmatic de?ays. Further corrective actions were addressed in a Tank Waste Remediation System Tank Farm Resumption of Work Plan, dated September 13, 1993. Contained within the Work Plan is the Integrated Schedule for reinitiating individual work activities on an ongoing basis.

Testing of the SY-101 mixer pump continues to be successfully accomplished. Many other tank farm work activities have now been fully resumed. In addition, reorganization is taking place to reduce management layers, and the Integrated Upgrade Plan of Action was issued January 31, 1994.

\section{Criticality Safety Issues}

On April 30, 1992, an Unreviewed Safety Question (USQ) concerning criticality safety issues in the Tank Farms was declared to be a reportable event. Unusual Occurrence report RL-WHC-TANKFARM-1992-0037 was issued. A prohibition was placed on all waste transfers into and between the tank farm facilities which is negatively affecting various Hanford progr ns. On September 1, 1992, the approved Justification For Continued Ope tions (JCO) was received. This establishes the limitations for all tanks re iving transfers and also excludes any interim stabilization of single-shell tanks until further evaluations are completed and approved by DOE-HQ. On December 15, 1992, the approved JCO was issued as WHC-SD-WM-JCO-001, "Justification for Continued Operations of Hanford High Level Waste Tanks Resulting From the Criticality USQ, 492-CRITSAS."

On March 31, 1994, DOE approved the WHC request to close the criticality USQ. This approval does not resolve the outstanding criticality safety issues pending in the tank farms. Resolution of these issues will require WHC to complete actions described in "Upgrade Activities for the Criticality Safety Program of Hanford High-Level Radioactive Waste Tank Farm, " dated September 1992.

Also, the existing JCO will remain in effect until WHC completes the following: 1) Criticality Safety Evaluation Reports for single-shell and double-shel1 tanks; 2) Criticality Prevention Specifications; 3) Criticality 
operating procedures; and 4) Operator training for the revised operating procedures.

6. Ten Single-She11 Tanks Added to Organic Salts Watch List

Ten tanks (A-101, AX-102, C-102, S-111, SX-103, TY-104, U-103, U-105, U-203, and U-204) were added to the Organic Salts Watch List in May 1994, based on a reassessment of the historical records on tank waste contents. Six of these tanks, (A-101, S-111, SX-103,.TY-104, U-103, and U-105) are also on other Watch Lists.

\section{Tank SY-101 Kydrogen Mitigation}

The hydrogen mixer pump installed in 241-SY-101 in July 1993, was operated 13 times during May 1994. The pump continues to maintain the gas inventory at historicaliy low levels as measured by the surface level. The pump operating mode has shifted from testing to Long Tarm Operations (surface level maintenance) where the objective is to maintair, the surface level less than 405 inches.

\section{Occurrence Reports}

RL-WHC-TANKFARM-1994-0002 (OFF-NORMAL) - SOIL LOADING OF WASTE TANK 241SY-101 EXCEEDS OPERATIONS SPECIFICATION DOCUMENT (OSD) OSD-T-151-00007 LIMIT (10-Day Report. [latest]. 5/06/94)

On January 8, 1994, a tank dome survey for SY-101 was performed to determine the amount of soil and gravel on top of the dome. The results of the survey were forwarded to Waste Tank Operations (WTO) personnel on January 10, 1994.

On January 11, the survey results were reviewed and determined to be a violation of OSD-T-151-00007 had occurred in relation to soil loading for this tank. Note: There are no Operational Safety Requirements (OSR) for tank dome loading. There are 3 limits specified in the OSD, when the three are added together, they result in a violation of the first level of control.

Immediate actions were taken to restrict vehicle access to the SY Tank Farm until concurrence from Waste Tank Plant Engineering can be obtained.

In the 10-day report dated February 24, 1994, further investigation was indicated as to the cause of why no further safety review was conducted prior to the gravel addition.

On May 5, 1994, MHC conducted an extensive follow-on review to resolve differing staff opinions as to whether conditions were within the Authorization Basis. It was concluded that these conditions do involve a USQ.

The current excess soil depth does not overload the tanks. Operation of the tanks, including the hydrogen mitigation mixer pump operation, can continue to operate as long as any additional loads do not exceed the remaining load margins. 
Immediate actions include surveying double-shell and single-shell tanks to verify soil cover depths in both East and West areas. Resolution of the USQ was submitted in May to clarify that the limit is the total load on the tank and not on the sources of that load.

9. Changes to the Monthly Report

Figure B-6 "Overall Waste Flow, Hanford Tank Waste Disposal" has been added to Appendix B "Double-Shell Tank Waste Type and Space Allocation" (see page $B-9$ ). 
The Watch Tanks pocket list has been revised to include 10 tanks added to the Organics Watch list in May 1994. Cut out the list and fold it. Tape it closed if you wish.

You are encouraged to urge your fellow employees to replace their obsolete pocket card with the list below. You may duplicate this list or cc:mail the author for additional copies.

The list will be updated and included in this report as changes occur.

\begin{tabular}{|c|c|c|c|c|}
\hline \multirow{3}{*}{ 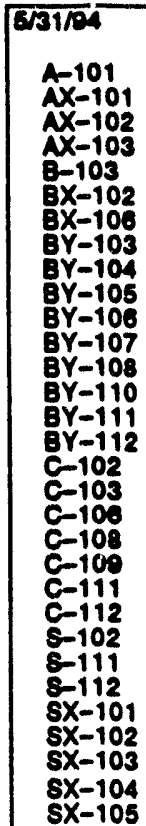 } & \multirow{3}{*}{ 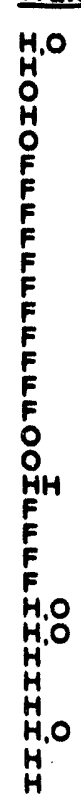 } & \multirow{3}{*}{ 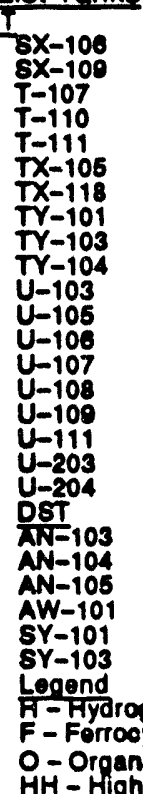 } & \multirow{2}{*}{$\begin{array}{l}H . O \\
H . O \\
F \\
H \\
O \\
O \\
O \\
F, O \\
F \\
F, O \\
F, O \\
H . O \\
H . O \\
O \\
O, H \\
., H \\
H \\
O \\
O \\
O\end{array}$} & 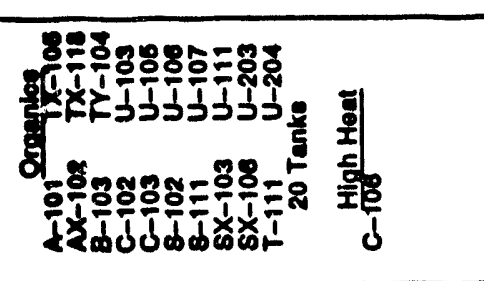 \\
\hline & & & & 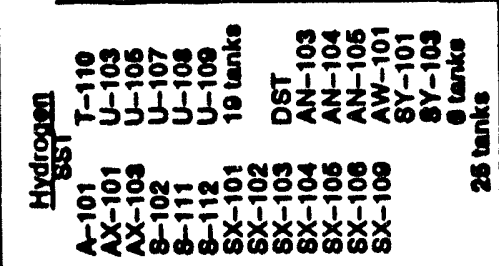 \\
\hline & & & 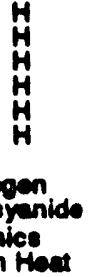 & \\
\hline
\end{tabular}


WHC-EP-0182-74

This page intentionally left blank 
WHC-EP-0182-74

APPENDIX A

WASTE TANK SURVEILLANCE MONITORING TABLES

A-1 


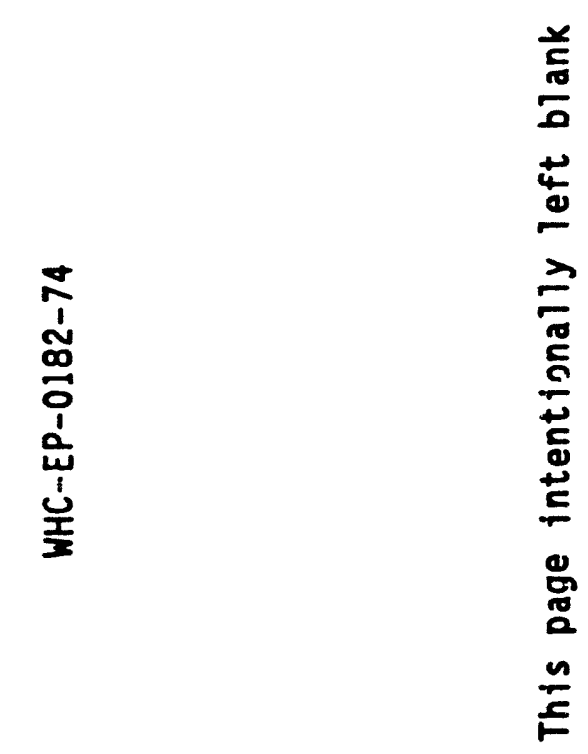




\section{TABLE A-1. WATCH LIST TANKS (Sheet 1 of 2)}

These tanks have been ldentlifed as Wetch Lbat Tanks in accordance with Public Law 101-510, Section 3137, - Safety Measures for Waste Tanks at Hanford Nuclear Peservation," (1990). These tanks have been identifled as the Priorty 1 Hanford Shte Tank Farm Safety lseves: "lssues/stuztlons that contain most necessary conditions that could lead to worker (onste) or oftstte radlation exposure through an uncontrolled release of fisston products, e.e. Tank SY-101."

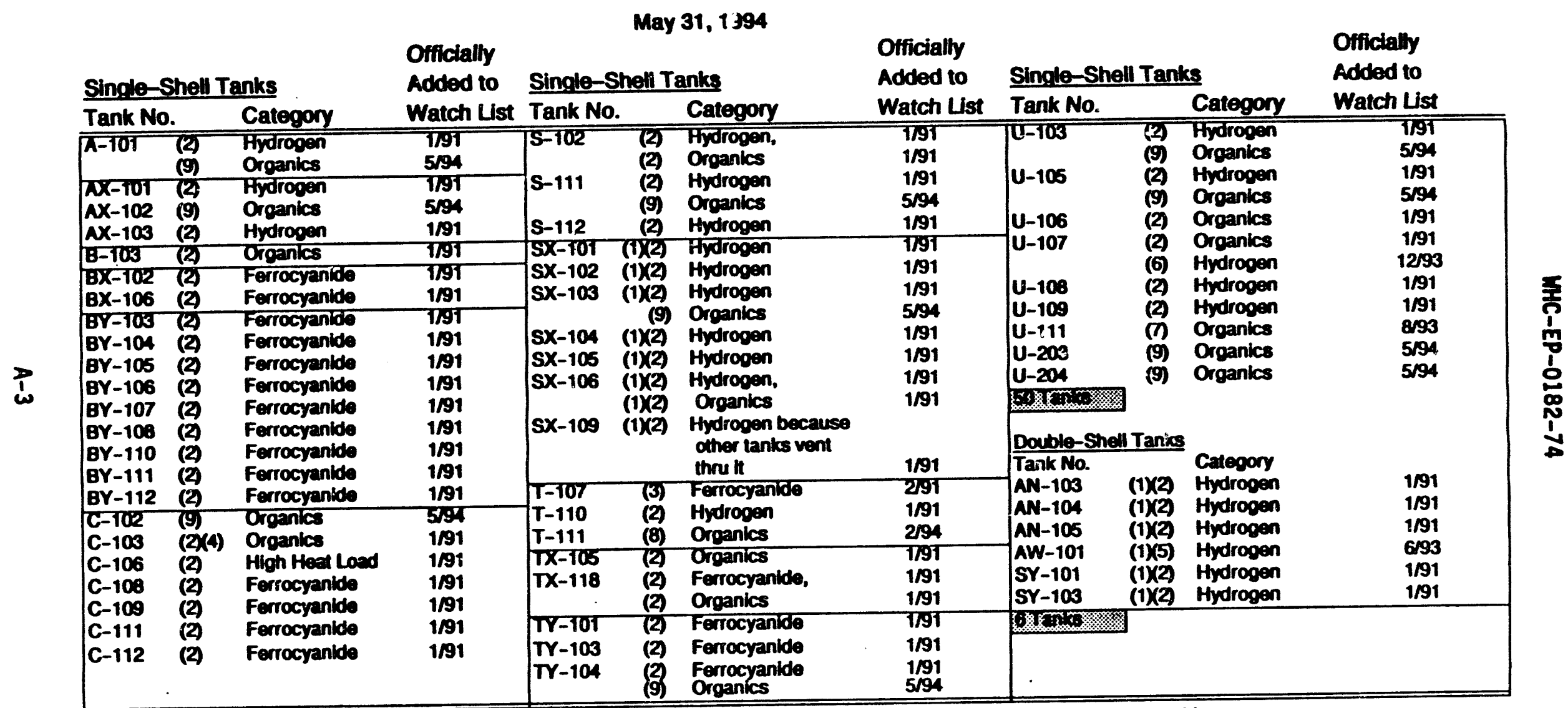

Ten tanks (A-101, S-102, S-111, SX-103, SX-106, TX-118, TY-104, U-103, U-105, and U-107, ) are on more than one Watch List

See footnotes next page 


\section{TABLE A-1 WATCH LIST TANKS (Sheet 2 of 2)}

Footnotes:

(1) These aight single-shell tanks and the six double-shell tanks on the Watch List are actively ventilated.

Atthough on various dates beginning in March 1990, WHC identified tanks containing ferrocyanide, organic salts, etc., which were then added to this report as Watch List tanks, the following official notifications were made to DOE-RL: Letter 9059124, H. D. Harmon, WHC, to R. E. Gerton, DOE-RL, "Safety Measures for Waste Tanks at Hanford Site, Richland, Washington," dated January 8, 1991, identified 23 ferrocyanide tanks, 23 tanks with potential for accumulation of flammable gas, elght organic tanks, and one high heat load tank, as being Watch List tanks. (52 tanks)

The ferrocyanide and hydrogen tanks were declared Unresolved Safety Questions (USQ); the organic tanks and the high heat load tank were within the safety envelope as defined by the safety analysis reports and were not designated as USQs. On March 1. 1994, the ferrocyanide USQ was closed. See Table A-2 footnote (1) for further information.

Letter '5059124.1 (revision to 9059124 above), dated February 8, 1991, added T-107 to the Ferrocyanide Watch List. (53 tanks)

Tank C-103 was dectared a USQ per Unusual Occurrence Report RL-WHC-TANKFARM-1992-0069, issued September 1992, because of an organic layer covering the surface. This USQ was closed May 19, 1994.

Letter 9354700, J. C. Fulton, WHC, to R. E. Gerton, DOE-RL, "Addition of Tank 241-AW-101 to Flammable Gas Watch List," dated June 3, 1993, added this double-shell tank to the Watch List. (54 tanks) recommended this tank be Included on the Organic Tanks Watch List. This tank was added to the Watch List on August $31,1993$.

Tank U-107 was declared a USQ per Occurrence Report RL-WHC-TANKFARM-1993-0115, issued December 1993, because of an increase in slurry growth. This tank is also on the Organics Watch List.

(8) Tank T-111 was added to the Organic Salts Watch List on February 28, 1994, upon recommendation by WHC Waste Tank Safety Program.

Ten tanks (A-101, AX-102, C-102, S-111, SX-103, TY-104, U-103, U-105, U-203, and U-204) were added to the Organic Salts Watch List, upon recommendation by WHC to DOE-RL, (Letter 9453328, M. A. Payne, WHC, to R. E. Gerton, DOE-RL, "Revision of the Organic Tanks Watch List," dated May 15, 1994,) and DOE-RL concurrence (Letter 94-SST-116, R. E. Gerton, DOE-RL, to President, WHC, Same Subject, dated May 25, 1994). Six of these tanks were already on the Watch List. 


\section{TABLE A-2. TANKS CONTAINING >1000 GRAM MOLE OF FERROCYANIDE (Watch List Tanks)(Sheet 1 of 2)}

The Unreviewed Salety Question (USO) assoclated with these tanks was closed March 1, 1994. (1)

Temoeratures in these tanks did not exceed the maximum temperature criteria for May 1994.

All Watch List tanks are reviewed for increasing temperature trends. Tanks conr ected to TMACS are required to be monitored continuousty: those not connected are required to be monitored weekly. Temperatures are taken in the waste unless indicated otherwise.

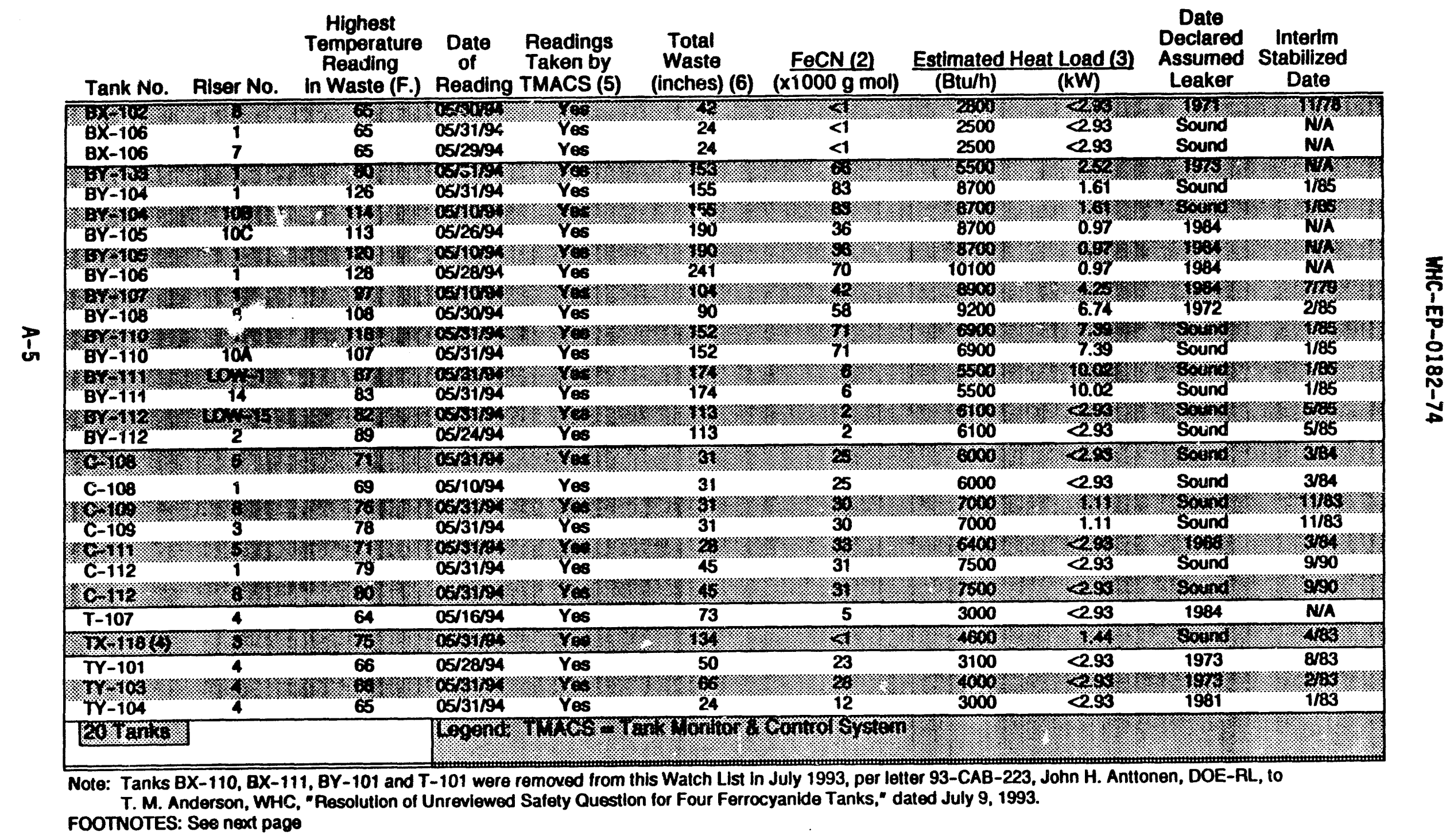


TABLE A-2. TANKS CONTAINING >1000 GRAM MOLE OF FERROCYANIDE (Watch List Tanks) (Sheet 2 of 2)

\section{FOOTNOTES:}

(1) Closure of the Ferrocyanide Unreviewed Safety Question (USQ) was approved in U.S. DOE Memorandum EM-36, Thomas P. Grumbly, to Manager, DOE Richland Operations Office, "Approval of the Request to Close the Ferrocyanide Unreviewed Safety Question at the Hanford High-Level Waste Tank Farms," dated March 1, 1994; and DOE-RL letter 94-SST-052, T. R. Sheridan, to President, WHC, "Closure of the Ferrocyanide Unreviewed Safety Question," dated March 4, 1994.

(2) The amount of FeCN reported in the tanks is based on WHC-SD-WM-ER-133-REV 0, "An Assessment of the Inventorles of the FeCN Watch List Tanks," (Table 3-7), October 1991.

(3) The estimated heat generation rates are from WHC-EP-0709, "Estimatation of Heat Load in Waste Tanks Using Average Vapor Space Temperatures," December 1993. This document analyzed all ferrocyanide tanks.

(4) This tank also contains a high concentration (>3\% wt TOC) of organic salts.

(5) This column indicates which tanks are being monitored by the Tank Monitor \& Control System (TMACS); automatic temperature readings are taken continuously. Temperatures in tanks connected to TMACS but temporarily not being monitored by TMACS are taken manually.

(6) Total waste in Kgal taken from Table E-5, Inventory and Status by Tanks for SSTs. Kgal/inches calculations for the temperature tables are as follows: (waste in inches is an approximation only for these temperature tables)

$$
\frac{\text { Kgal waste }-12.5 \mathrm{Kgal} \mathrm{waste*}}{2.75 \mathrm{Kgal} / \mathrm{inch}}+12 \text { inches* }
$$

- The bottom 12 inches in dish bottom tanks contain $12.5 \mathrm{Kgal}$. All tanks are calculated as dish bottom tanks for the temperature tables, although A and $\mathrm{AX}$ farms have it bottoms. Inches are from centerline tank bottom. 


\section{TABLE A-3. TANKS WITH POTENTIAL FOR HYDROGEN OR FLAMMABLE GAS ACCUMULATION ABOVE THE FLAMMABILITY LIMIT (Watch List Tanks)}

These tanks have en Unreviemed Safety Question (USO) because of the potentlal consequences of a radiological release resulting from a flammable gas burn, an event not analyzed in the SST Safety Analysis Report.

Temperatures in these tanks did not exceed the applicable maximum temperature criteria for the month of May 1994. All Watch List tanks are reviewed for increasing temperature trends. Temperatures are taken in the waste unless indicated otherwise.

\begin{tabular}{|c|c|c|c|c|c|c|c|}
\hline Tank No. & Riser No. & $\begin{array}{c}\text { Highest } \\
\text { Temperature } \\
\text { Reading (F.) } \\
\text { in Waste } \\
\end{array}$ & $\begin{array}{c}\text { Date } \\
\text { of } \\
\text { Reading } \\
\end{array}$ & $\begin{array}{c}\text { Total } \\
\text { Waste (3) } \\
\text { (inches) }\end{array}$ & $\begin{array}{l}\text { Monitoring } \\
\text { Frequency }\end{array}$ & $\begin{array}{c}\text { Assumed } \\
\text { Leaked } \\
\text { Date } \\
\end{array}$ & $\begin{array}{c}\text { Interim } \\
\text { Stabilized } \\
\text { Date }\end{array}$ \\
\hline$\overline{\alpha-101(2)}$ & R-12 & 154 & 0578194 & 354 & Weeky & \$OUND & N/K \\
\hline $1 \% 16 \%$ & $x$ & 䫶 8. & ongos & $2 \%$ & w & Kesty & 縈H \\
\hline$A x-103$ & R-13c & 111 & $05118 / 94$ & 48 & Weokly & SOUNb & $8 / 87$ \\
\hline 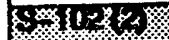 & 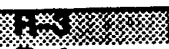 & 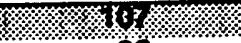 & $4 x^{-1} p^{p}$ & 34 & 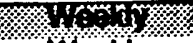 & 㗈 8198 & X' \\
\hline $5-111(2)$ & R-4 & 92 & $05 \sqrt{30 / 94}$ & 224 & Weokly & SOUND & NNA \\
\hline 5 $8 \%$ & 瓷 & $x$ & otross & 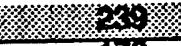 & Hos & $20 \%$ & 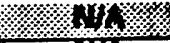 \\
\hline $5 x-101(5)$ & A-15 & 138 & Co5123/s4 & 173 & Weokly & SOUND & W/h \\
\hline or & 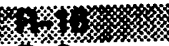 & wo & $800 x$ & 4 & Whys & xysk & Wh \\
\hline $5 x-103(2)$ & $R-2$ & 174 & 05130194 & 245 & Weokly & SOUNb & NA \\
\hline $5 \%$ & 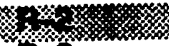 & xyon & $6+3 \times 3$ & $x$ & 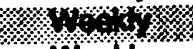 & KKn & Ho \\
\hline $5 x-105$ & $R-2$ & 180 & $05 \sqrt{23 / 93}$ & 256 & Weakly & SOOND & NA \\
\hline $\cos 10 \%$ & $x$ & W & 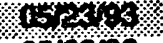 & $<$ & wh & so & 14 \\
\hline $5 x-109(1)$ & $A-10$ & 148 & $0.5 / 23193$ & 98 & Weekly & 1905 & $5 / 81$ \\
\hline xos & 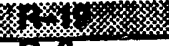 & 皿 & $80 \cos x$ & 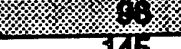 & whs & 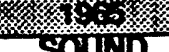 & stig \\
\hline $1-110$ & h-8 & 63 & Cosisorst & 745 & Weokly & SoUND & \\
\hline $36<<$ & 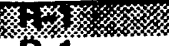 & \% & 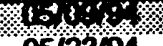 & 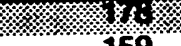 & 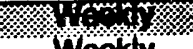 & 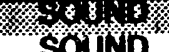 & N/A \\
\hline $0-105(2)$ & R-1 & 89 & $05 / 23 / 94$ & 159 & Woekly & SOUND & $\begin{array}{l}\text { N/n } \\
\text { sery }\end{array}$ \\
\hline Hors & 禁奴 & 6 & $6 \operatorname{sen}^{2}$ & 178 & \%oin & SOUND & NA \\
\hline $0-108$ & $\mathbf{R}-\mathbf{1}$ & 88 & $05102 / 94$ & 178 & Weekly & SOUND & Wh \\
\hline $\begin{array}{l}13 \\
11-103\end{array}$ & 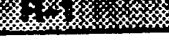 & $\sqrt{13}$ & $\frac{10428}{051894}$ & 80 & Weaky & SOUND & NA \\
\hline $\begin{array}{l}A i-103 \\
4\end{array}$ & & w & 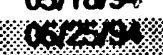 & & wots & xorts & 4 \\
\hline$A N-105$ & & 114 & $05 / 10194$ & & Weekly & SOUND & $\mathbf{N} \mathbf{A}$ \\
\hline 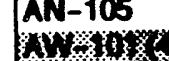 & & 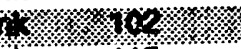 & ros $;$ & & $w \%$ & soy & 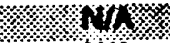 \\
\hline SY-101 & . Wher 170 & 115 & $05131 / 94$ & & Dally & SOUND & Nis \\
\hline $3 x-10 \%$ & 荻 & x & 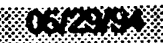 & & $\mathbf{D}$ & xosys & $4 y$ \\
\hline$S Y-103$ & & 97 & $05 / 25 / 94$ & & Weokly & SOUND & NA \\
\hline & & & & & & 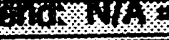 & Cabis: \\
\hline
\end{tabular}

(1) Tank SX-109 has the potential for flammable gas accumulation only because other SX tanks vent through ht.

(1) Tank SX-109 has the potentla for Tam SX-106, U-103, U-105 and U-107 are also on the Organics Watch List

(2) Tanks A-101, S-102, S-111, SX-103, SX-106, U-103, U-105 and

(3) See lootnote (G) In Table A-2 (Ferrocyanide Tanks) for Total Waste/inches calculailons. Wos. in -RL. "Addition of Tank 241-AW-101 to Flammable

(4) Tank AW-101 was added to this list per lot

Gas Watch List," dated June 3, 1993.

(5) Tank $U-10$
growth. 


\section{TABLE A-4. SINGLE-SHELL TANKS CONTAINING CONCENTRATIONS OF ORGANIC SALTS $>3$ WEIGHT \% TOTAL ORGANIC CARBON (TOC) (Watch List Tanks) (Sheet 1 of 2)}

These tenke hove are

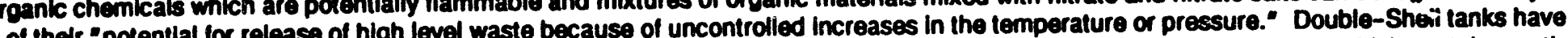
lisied here because of their "potentlal lor release of high level waste because of uncontrolled increases in the temere is no credible organic safety concern for tanks which contaln mostly liquld. $>3$ Weight \% TOC and are not on the Watch List because they contaln mostly liquld and there is no credible organle salety concert and heat up, and "high organic concentrations in the tanks could support an

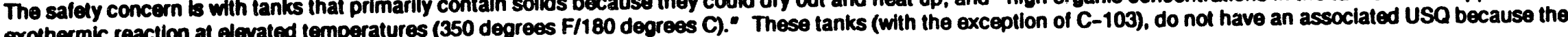
exothermic reaction at elevated temperatures (350 degrees Safety Analysis Report.

Temperatures in these tanks did not exceed the apolicable maximum temperature criteria for the month of Mar 1994. These tanks are monitored woekly. All Watch List tanks are reviewed for increasing temperature trends.

Temperatures are taken in the waste unless indicated othenwise.

NanO 3

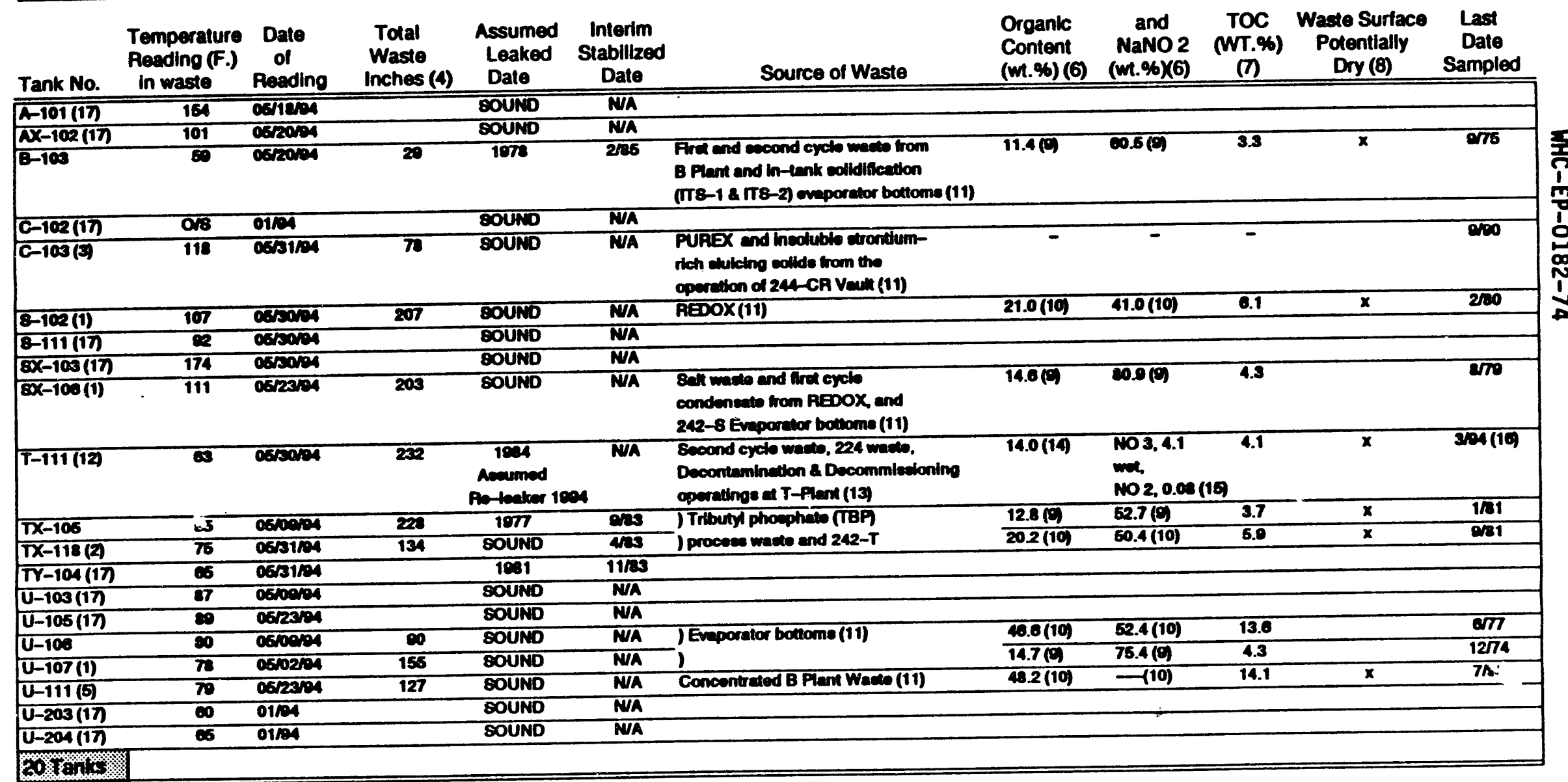

See Footnoles next pages 


\section{TABLE A-4. SINGLE-SHELL TANKS CONTAINING CONCENTRATIONS OF ORGANIC SALTS $>3$ WEIGHT \% TOTAL ORGANIC CARBON (TOC) (Sheet 2 of 2)}

\section{Footnotes:}

(1) These tanks also have the potentlal for hydrogen or flammable gas accumulation.

(2) Tank TX-118 also contalns ferrocyanide.

(3) Tank C-103 was declared a USQ because of an organic layer covering the surface, reference Unusual Occurrence Report RL-WHC-TANKFAPM1992-0069, kssued September 1992.

(4) See footnote (6) in Table A-2 (Ferrocyanide Temperature Table) for Total Wastelnches calculations. Waste inches cakculations are approximations only for temperature tables.

(5) Tank U-111 was added August 31, 1993. See August 1993 Summary Highlights for information and Table A-1. "Watch List Tanks" for applicable reference.

(6) WHC, 1990 "The Kyshtym Explosion and Exploston Hazards with Nitrate-Nitrtte Bearing Wastes with Acetates and Other Organic Salts," WHC-SD-LB-033, Westinghouse Hanford Company, Richland, Washington

(n) Dry w..\% basts. Calculated as w.\% sodium acetate equivalent X.2928.

(8) Dus 10 absence of supernatant liquid.

(9) Calculated from data developed by Track Radioactive Components (TRAC) computer code, 1984.

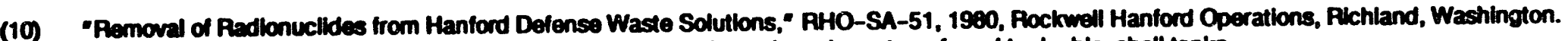
All of part of liquld from which compostion data were derived may have teen transferred to double-shell tanks.

(11) WHC. 1993, "Action Plan for Responses to Abnormal Conditions in Hanford Site Radioactwe Waste Tanks with High Organic Content." WHC-EP-0461, Rev. 1, Westinghouse Hanford, Richland, Washington.

(12) Tank 241-T-111 was added to the Organic Salts Watch List on February 28, 1994, upon recommendation by WHC Waste Tank Safety Program. WHC, 1990, "A Hibtory of the 200 Area Tank Farms," WHC-MR-0132, Westinghouse Hanford, Richland, Washington.

(14) Pacific Northwest Laboratorles analysis on Core 33, Segment 2, dated January 14, 1994.

(15) WHC, i993, "Single-Shell Tank Characterzation, Tank T-111, Cores 31 and 33," WHC-SM-PD-024, Rev OA, Westinghouse Hantord, Richland, Washington.

(16) Data not yot avallable. 
TABLE A-5. SINGLE-SHELL TANKS WITH HIGH HEAT LOADS (40,000 Btu/h)(Sheet 1 of 2)

High heat load tanks have temperature survelliance requirements establtshed by SD-WM-SAR-006 REV 1. "SST Isolation Safety Anatysis Report," dated January 1986, and OSD-T-151-00013 REV D-O, "Operating Specifications for Single-Shell Waste Storage Tanks," dated August 1990. While all of these tanks are considered high heat load tanks per SAR definition, only one (241-C-106) is on the High Heat Watch List.

Temperatures in these tanks did not exceed SAR or OSD requirement limits for the month of May 1994. All high heat load tanks are on active ventilation unless indicated otherwise in the footnotes. These high heat tanks are reviewed for increasing temperature trends.

Temperatures are taken in the waste unless indicated otherwise.

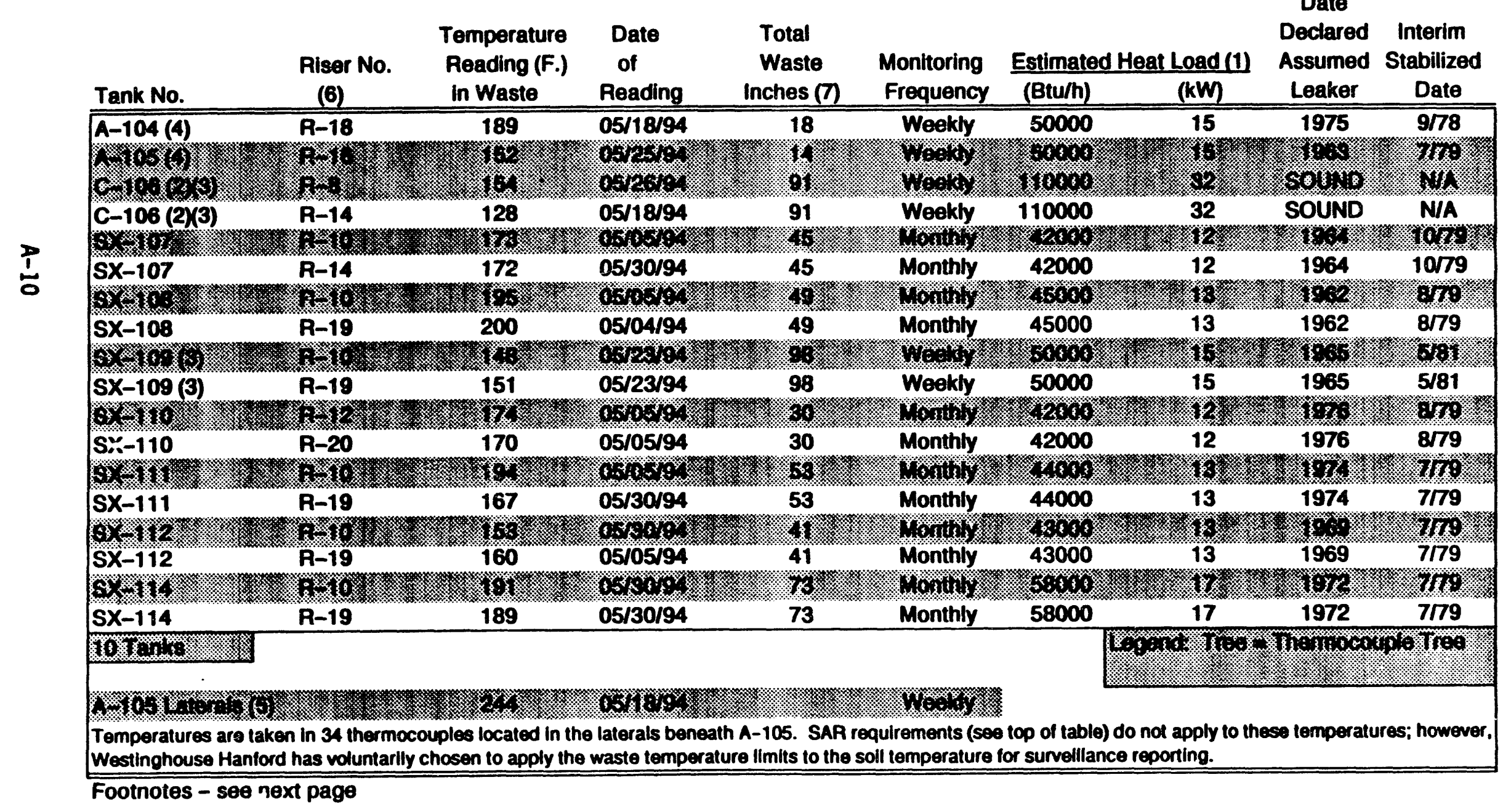




\section{TABLE A-5. SINGLE-SHELL TANKS WITH HIGH HEAT LOADS $(\mathbf{4 0 , 0 0 0}$ Btuth)(Sheet 2 of 2)}

\section{Footnotes:}

(1) Hloh heat and SR 90 (hall life of 28.1 years). Tank C-105 was re-evaluated in WHC-SD-WM-ER-189. "Thermal Analysis of Tank 241-C-105 in Support of Process Test." and SR 90 (half life of 28.1 years). Tank C-105 was re-evaluated in WHC-SO-Wm-ER-189, Thermal A Halys Heat Load to Normal, effecthe July 1. 1993. January 1993. Engineering Change Nolice 196834 , June 24, 1993, Changed the stalus of C-105 Irom Huring the ventllation outage in 1992 . WHC-SD-WMTank C-106 was re-evaluated using a revised thermal history based on the thermai transient behavior during the ventllatlon outage in 110,000 Btu/hr ( $+1-20,000$ Btu/h) tor this tank.

(2) Perlodic water additions are required in C-106 to maintain evaporative cooling and thus prevent overheating. This tank is scheduled for partial retrieval Periodic water addilons are requied in C-10s to malns will be discontinued. Temperatures in riser 8 are consistent at mid-150 degrees, however, starting in 1997, at which time cooling water additons will be discontlisued. Temperalures Starting March 7, 1994, C-106 is underooing a llquid-reduction process test temperatures in riser 14 vary between approxlmately 20 and 135, also consistently. Staning completed in FY-94. Cooling water is not added during the test. C-Farm thermocouple trees were connected to the Tank Monitor and Control System (TMACS) and are monitored continuousty.

(3) Watch List Tanks: C-106 is on the high heat load Watch List because in the event of a leak "without water additlons the tank could exceed structural temperature limits resulting in unacceptable structural damage."Also $5 e 0$ (2) above. SX-109 is on the hydrogen Watch List becuase it has the potential for flammable gas accumulation due to other SX tanks venting through it.

(4) A-104105/106 exheuster has been out of senvice from October 1, 1991, untll August 20, 1992, when Ht was briefly restarted. Problems exist which must be resolved before the exhauster boperational. Temperatures in A-104 and A-105 are monitored weekly.

5) Maximum interal temperatures under A-105 increased 20 degrees F. by January 1992, but then dropped a few degrees and have remained lairty stable at current temperature. These temperatures are monitored weekly.

(6) Tanks A-104 and A-105

Two temperature probes are inetelled in risers in A-104, and six are installed in risers in A-105. These are indluldual probes. In A-104, the probes are in contact with the studoe; in $A-105$, they are in contact with the bottom of the tank (A-105 has a bulged bottom).

Tank C-106

Tank C-106 has stx functioning thermocouples ( $\$ 11$ through $\emptyset$ ) on riser 8, and 12 functioning thermocouples on riser 14 . The trees arel connected to TMACS and are monitored continuously.

Tanks SX-107, 108, 109, 110, 111, 112, and 114

Each of these tanks has elght thermocouple trees, with elght thermocouples on each tree, with the exception of SX-108, which has four operational thermomach

(n) Cakculations for Total Waste Inches: see footnote (๗). Table A-2 (Ferrocyanlde Tanks). Waste in inches is an approximation only for temperature tables.

(8) There are 19 single-shell tanks with active ventllatlon (elght are on the Watch List as indicated by an asterisk):

$\begin{array}{cll}A-104 & S X-101: & S X-107 \\ A-105 & S X-102: & S X-108 \\ A-106 & S X-103: & S X-109 \\ C-104 & S X-104: & S X-110 \\ C-105 & S X-105: & S X-111 \\ C-106 & S X-106 & S X-112 \\ & & S X-114\end{array}$


TABLE A-6. NON-WATCH LIST LOW HEAT LOAD TANKS $(<40,000 \mathrm{Btu} / \mathrm{h})$

(Page 1 of 3)

Temperatures are taken semiannually in January and July, unless otherwise indicated, in the following 91 single-shell tanks. Legend follows table.

\section{Highest Temperatures}

$$
\text { taken in waste Total Waste (1) }
$$

Tank No. Jul. 93 Jan. 94 Kaal Inches

\section{Comments}

\begin{tabular}{|c|c|c|c|c|c|}
\hline$A-102$ & 92 & 87 & 41 & 15 & \\
\hline$A-103$ & 117 & 114 & 370 & 135 & \\
\hline$A-106$ & 137 & 135 & 125 & 50 & \\
\hline AX-104 & 98 & 92 & 7 & 2 & \\
\hline B-101 & 108 & 109 & 113 & 48 & \\
\hline $\mathrm{B}-102$ & 63 & 63 & 32 & 19 & TCli1 O/S, reading in vapor space \\
\hline$B-104$ & 66 & 65 & 371 & 142 & \\
\hline $8-105$ & 66 & 65 & 306 & 50 & \\
\hline 8-106 & 62 & 63 & 117 & 67 & TCH1 \& $20 / S$ \\
\hline B-107 & 62 & 60 & 165 & 41 & TC"1 1 thru 3 O/S, reading in vapor space \\
\hline $\mathrm{B}-108$ & 62 & 64 & 94 & 54 & \\
\hline B-109 & 61 & 63 & 127 & 97 & \\
\hline $8-110$ & 68 & 63 & 246 & 94 & TC"il thru 4 O/S. Reading in vapor space \\
\hline $\mathrm{B}-111$ & 86 & 86 & 237 & 19 & TC"\#1 \& 2 O/S, work order issued, historical readings erratic \\
\hline $\mathrm{B}-112$ & 63 & 64 & 33 & 7 & \\
\hline B-201 & 60 & 60 & 29 & 151 & \\
\hline B-202 & 61 & 60 & 27 & 141 & \\
\hline $8-203$ & 62 & 61 & 51 & 263 & \\
\hline$\overline{B-204}$ & 62 & 61 & 50 & 258 & \\
\hline$B X-101$ & O/s & O/S & 43 & 27 & All TCs O/S, work order lesued, last reading 74 F. in 11/92 \\
\hline BX-103 & $\mathrm{O} / \mathrm{S}$ & O/S & 66 & 31 & All TCs O/S, last reading $77 \mathrm{~F}$. in 10/92 \\
\hline \multirow[t]{2}{*}{$8 \mathrm{~B}-104$} & O/S & O/S & 99 & 43 & (2) No TC tree per Riser Configuration document \\
\hline & & & & & Last reading $87 \mathrm{~F}$. in $10 / 80$ \\
\hline BX-105 & 63 & 66 & 51 & 26 & \\
\hline BX-107 & O/S & O/S & 345 & 133 & All TCs O/S, work order issued, last reading $69 \mathrm{~F}$. in $10 / 82$ \\
\hline$\overline{B X-108}$ & 63 & 65 & 26 & 17 & \\
\hline BX-109 & 77 & O/S & 193 & 78 & Temp reading obtained $7 / 93$ only, 2 attempts made 1/94 \\
\hline & & & & & No historical readings available \\
\hline $\mathrm{BX}-110$ & 68 & 74 & 199 & 80 & Readings taken weekly, pumping began, now on hold \\
\hline $8 x-111$ & 65 & 69 & 211 & 84 & Readings taken weokly, tank beling pumped \\
\hline$B x-112$ & 62 & 65 & 165 & 67 & \\
\hline 30 BY-101 & 75 & 75 & 387 & 148 & Continuous readings taken on TMACS \\
\hline \multirow[t]{2}{*}{31 BY-102 } & Ors & O/S & 341 & 131 & (2) No TC tree per Riser Configuration document. \\
\hline & & & & & Last reading 72 F. $\ln 4 / 79$ \\
\hline BY-109 & O/S & O/s & 423 & 161 & (2) No TC tree per Riser Conflouration document. \\
\hline
\end{tabular}


TABLE A-6. NON-WATCH LIST LOW HEAT LOAD TANKS $(<40,000 \mathrm{Btu} / \mathrm{h})$ (Page 2 of 3)

Temperatures are taken semiannually in January and July, unless otherwise indicated, in the following 95 single-shell tanks. Legend follows table.

\section{Highest Temperatures}

$$
\text { taken in waste Total Waste (1) }
$$

Tank No. Jul. 93 Jan. 94 Kgal Inches

\section{Comments}

\begin{tabular}{|c|c|c|c|c|c|c|}
\hline \multirow{2}{*}{\multicolumn{7}{|c|}{$33 \quad C-101(3) \quad 88$}} \\
\hline & & & 86 & 88 & 39 & \\
\hline 34 & C-104(3) & 87 & 85 & 295 & 115 & TC\#1 thru 5 O/S, reading in vapor space \\
\hline 35 & $\mathrm{C}-105(3)$ & 92 & 80 & 150 & 63 & Monitored weekly, formerly on Hlgh Heat Load list \\
\hline \multirow[t]{2}{*}{36} & $\mathrm{C}-107(3)$ & 125 & 124 & 275 & 107 & Monthly reading requirement per procedure, monitored \\
\hline & & & & & & wookly \\
\hline 37 & C-110(3) & 65 & 66 & 187 & 75 & TCA1 thru 4 O/S, reading in vapor space \\
\hline 38 & $C-201(3)$ & 61 & 56 & 2 & 13 & \\
\hline 39 & $\mathrm{C}-202(3)$ & 61 & 60 & 1 & 8 & \\
\hline 40 & $\mathrm{C}-203(3)$ & 60 & 59 & 5 & 29 & \\
\hline 41 & C-204 & O/S & O/S & 3 & 18 & In-tank photos revealed no tree. Last reading obtained prior ' 91. \\
\hline 42 & S-101 & 115 & 118 & 427 & 162 & TC"1, 3,5, \&6 O/S, work order issued \\
\hline 43 & $s-103$ & 85 & 87 & 248 & 98 & \\
\hline 44 & $S-104$ & 104 & 108 & 294 & 114 & \\
\hline 45 & s-105 & 73 & 78 & 456 & 173 & \\
\hline 46 & s-106 & 78 & 81 & 543 & 205 & \\
\hline 47 & s-107 & 107 & 110 & 368 & 129 & \\
\hline 48 & s-108 & 85 & 89 & 604 & 227 & \\
\hline 49 & $s-109$ & O/S & 68 & 568 & 214 & Readings available on TC"7 only \\
\hline 50 & $\mathrm{~S}-110$ & 116 & 117 & 390 & 149 & \\
\hline 51 & $S x-113$ & 73 & $\pi$ & 26 & 15 & \\
\hline \multirow[t]{2}{*}{52} & $5 x-115$ & O/S & O/S & 12 & 10 & (2) No TC tree, per Riser Configuration document, \\
\hline & & & & & & last reading prior to $12 / 91$ \\
\hline 53 & $T-101$ & 66 & 72 & 102 & 45 & Formerly on ferrocyanide Watch Llst \\
\hline \multirow[t]{2}{*}{54} & $T-102$ & O/S & O/S & 32 & 19 & (2) No TC tree per Riser Configuration document, \\
\hline & & & & & & last reading $68 \mathrm{~F}$. in $2 / 81$ \\
\hline 55 & $T-103$ & 59 & 62 & 27 & 17 & \\
\hline 56 & $T-104$ & O/S & 62 & 445 & 169 & All TCs O/S, work order issued \\
\hline 57 & $T-105$ & O/S & $\mathrm{O} / \mathrm{S}$ & 98 & 43 & (2) No TC tree per Riser Configuration document \\
\hline 58 & $T-106$ & 59 & 60 & 21 & 15 & \\
\hline 59 & $T-108$ & Ors & 57 & 180 & 73 & \\
\hline 60 & $T-109$ & O/s & O/S & 58 & 29 & All TCs O/S, work order issued, last reading 75 F. In $2 / 91$ \\
\hline 61 & $T-112$ & 58 & 60 & 67 & 32 & \\
\hline 62 & $T-201$ & 59 & 60 & 29 & 150 & \\
\hline 63 & $T-202$ & 58 & 62 & 21 & 110 & \\
\hline 64 & $T-203$ & 75 & 64 & 35 & 182 & \\
\hline 65 & $T-2 C_{i}$ & 60 & 63 & 38 & 197 & \\
\hline 66 & TX-101 & O/S & Ors & 87 & 39 & (2) No TC tree per Riser Conflguration document \\
\hline 67 & TX-102 & O/S & O/S & 113 & 49 & Cable cut from tree \\
\hline
\end{tabular}


TABLE A-6. NON-WATCH LIST LOW HEAT LOAD TANKS $(<40,000 \mathrm{Btu} / \mathrm{h})$

(Page 3 of 3)

Temperatures are taken semiannually in January and July, unless otherwise indicated, in the following 95 single-shell tanks. Legend follows table.

\section{Highest Temperatures}

taken in waste Total Waste (1)

Tank No. Jul. 93 Jan. 94 Kgal Inches Comments

\begin{tabular}{|c|c|c|c|c|c|}
\hline$T X-103$ & 55 & 71 & 157 & 54 & Cable cut from tree, readings taken by instrument Techniclan \\
\hline & & & & & Hloh reading taken $1 / 93$ was $71 \mathrm{~F}$. \\
\hline$T X-104$ & 60 & 65 & 65 & 31 & Cable cut from tree, readings taken by Instrument Techniclan \\
\hline $7 x-106$ & 59 & 78 & 453 & 172 & Cable cut from tree, readings taken by Instrument Techniclan \\
\hline$T x-107$ & 60 & 66 & 36 & 21 & Cable cut from tree, readinos taken by Instrument Techniclan \\
\hline $7 x-108$ & 61 & 68 & 134 & 56 & Cable cut from tree, readings taken by Instrument Technician \\
\hline \multirow{2}{*}{$7 x-109$} & 64 & 94 & 384 & 147 & Cable cut from iree, readings taken by Instrument Technician \\
\hline & & & & & High reading taken $1 / 93$ was $97 \mathrm{~F}$. \\
\hline$T x-110$ & O/S & O/S & 462 & 175 & Cable cut from tree, readings taken by Instrument Techniclan \\
\hline \multirow[t]{2}{*}{$T x-111$} & 61 & 79 & 370 & 142 & Cable cut from tree, readings taken by Instrument Technician \\
\hline & & & & & High reading taken $1 / 93$ was $73 \mathrm{~F}$. \\
\hline $\mathrm{TX}-112$ & 72 & 67 & 649 & 243 & Cable cut from tree, readings taken by Instrument Technician \\
\hline$T x-113$ & 65 & 72 & 607 & 228 & Dial pushed inside housing, readings taken by Instr. Tech. \\
\hline $7 x-114$ & O/S & O/S & 535 & 202 & Cable cut from TC tree \\
\hline$T X-115$ & 67 & 70 & 640 & 240 & Diai pushed inside housing, readings taken by Instr. Tech. \\
\hline$\overline{T x}-116$ & O/S & O/S & 631 & 237 & (2) Tree cut off in riser per Aiser Configuration document \\
\hline $\bar{X}-117$ & $\mathbf{O} / \mathbf{S}$ & O/S & 626 & 235 & All TCs O/S, cable cut from tree \\
\hline$T Y-102$ & O/S & 60 & 64 & 31 & \\
\hline$\overline{T Y}-105$ & $\pi$ & 79 & 231 & 91 & \\
\hline$\pi-106$ & 60 & 59 & 17 & 14 & \\
\hline $\mathrm{U}-101$ & 61 & 67 & 25 & 17 & \\
\hline $\mathrm{U}-102$ & 81 & 85 & 374 & 143 & \\
\hline $\mathrm{U}-104$ & O/S & O/S & 122 & 52 & (2) No TC tree per Riser Conflouratlon document \\
\hline $\mathrm{U}-110$ & 72 & 76 & 186 & 75 & \\
\hline U-112 & 61 & 63 & 49 & 25 & \\
\hline U-201 & O/S & 61 & 5 & 29 & \\
\hline$U-202$ & 59 & 61 & 5 & 29 & $\dot{-}$ \\
\hline
\end{tabular}

(1) seo Table A-2 (footnote 6 ) for waste gallons/inches calculations.

(2) Thermocouples in nine tanks (BX-104, BY-102, BY-109, SX-115, T-102, T-105, TX-101, TX-116, and U-104) are out of senvice due to no TC trees in these tanks, or the thermocouples have been cut off, covered over, or are othenwise unable to function, per the Riser Configuration document. (Aiso see comment section above)

(3) All TC trees in C-Farm connected to nMACS on 3/31/94, except for C-204 which has no tree.

(4) T-111 was deleted from this list and added to the Organics Watch Uat in February 1994

(5) AX-102, C-102, U-203 and U-204 were deleted from this list and added to the Organics Watch List in May 1994.

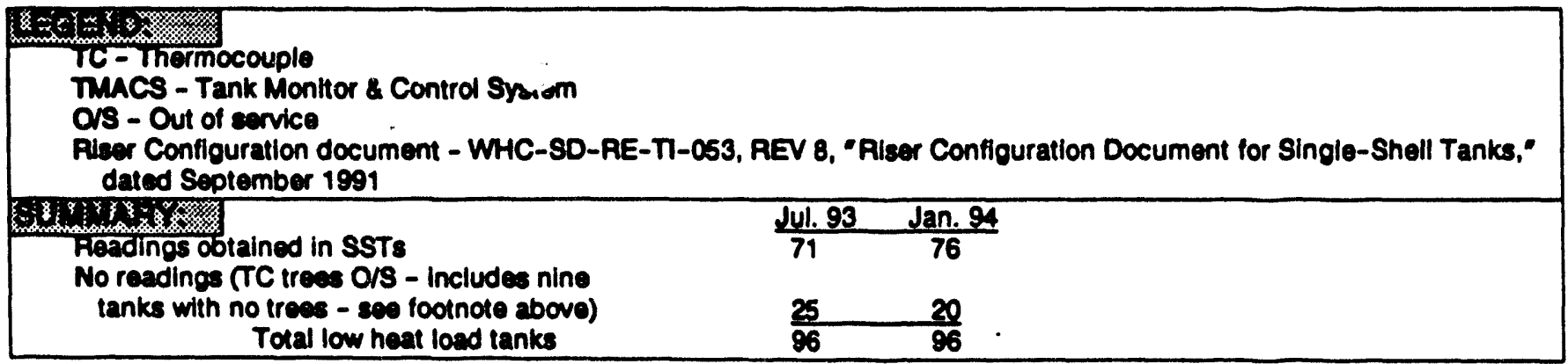




\section{TABLE A-7. SINGLE-SHELL TANKS MONITORING COMPLIANCE STATUS 149 TANKS (Sheet 1 of 5)}

The following table indicates whether Single-Shell tank monitoring was in compliance with the requirements as specified in the applicable documents as of the last day of the applicable month:

NOTE:

NI Watch Liat and High Heat tank temperature monttoring is in compliance. (5)

All Dome Eevation Survey monitoring is in compliance.

All Drywell monthoring is in compliance.

Peychrometrics (2)

In-tank Pholographs (3)

Precoure Montioring ( $(\theta)$

CAMMAMP Monitoring (n)

Vepor Monitoring (8)

Information as of May 31, 1994

\begin{tabular}{|c|c|c|c|c|c|c|c|c|c|}
\hline \multirow{4}{*}{$\begin{array}{l}\text { Tank } \\
\text { Number }\end{array}$} & \multirow{2}{*}{\multicolumn{2}{|c|}{ Category }} & \multirow{4}{*}{$\begin{array}{c}\text { Temperature } \\
\text { Peadings } \\
(5 \times())\end{array}$} & \multirow{3}{*}{\multicolumn{2}{|c|}{$\begin{array}{l}\text { Surface Level } \\
\text { Readings (1) } \\
(-357)\end{array}$}} & \multirow{4}{*}{$\begin{array}{l}\text { LOW } \\
\text { Rocedinge } \\
\text { (-357) } \\
\text { Noutron }\end{array}$} & \multicolumn{2}{|c|}{ Radiation Readings } & \multirow{4}{*}{$\begin{array}{l}\text { Dome } \\
\text { Eevation } \\
\text { Surveys } \\
\text { (OSPSAP) }\end{array}$} \\
\hline & & & & & & & \multirow{3}{*}{$\begin{array}{l}\text { Lateral } \\
\text { Readinges } \\
(-357)\end{array}$} & \multirow{3}{*}{$\begin{array}{l}\text { Drywell } \\
\text { Readings } \\
\text { (OSR/SAR) }\end{array}$} & \\
\hline & \multirow{2}{*}{$\begin{array}{l}\text { Watch } \\
\text { List(6) }\end{array}$} & \multirow{2}{*}{$\begin{array}{l}\text { High } \\
\text { Heat }\end{array}$} & & & & & & & \\
\hline & & & & M.T. & FIC & & & & \\
\hline$A-101$ & $x$ & & & & ons & & & 7 & \\
\hline$A-102$ & & & & WWt & & 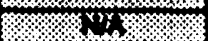 & & & 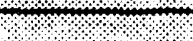 \\
\hline A-105 & & & צ' & $3 n \%$ & & \% & ক & X & $1 \%$ \\
\hline A-104 & & $\bar{x}$ & r & & m & \% & & & \\
\hline A-106 & & $\bar{x}$ & & & 36 & 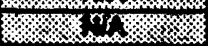 & & & \\
\hline$A-100$ & & & (x) & 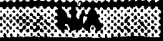 & 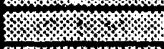 & 78 & r. & ఋ & r. \\
\hline DX-101 & $\bar{x}$ & & & 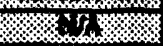 & & & & & \\
\hline$\sqrt{x-102}$ & & & & & . & \% & পে & স & \\
\hline$a x-105$ & $\bar{x}$ & & & $61 \%$ & & (n) & \% & \% \% & \\
\hline $2 x-104$ & & & & & 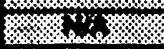 & (7. & থ & খ & $\sqrt{2}$ \\
\hline E-101 & & & & Wh & / & 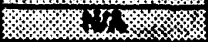 & থ/: & & \\
\hline E-102 & & & & 20 & \% & \%> & প. & স > & \% \\
\hline E-103 & $\bar{x}$ & & & 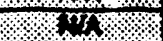 & \% & 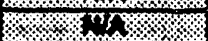 & থ: & স্যে ২: & \\
\hline E-104 & & & r & 16. & 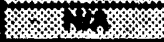 & m & ঋ & ঋ. & \\
\hline E-106 & & & & & 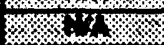 & & ২ে & \% & \\
\hline $0-100$ & & & & w' ' & \% & $2 \pi$ & 媇 & & r \\
\hline E-107 & & & \% & & \%: \%! & $8 \times 1 / 2$ & ক. & ख. & ( \\
\hline E-100 & & & m & $m \%$ & & 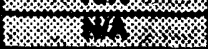 & স & & \\
\hline$E-100$ & & & 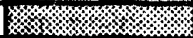 & / & 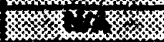 & 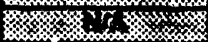 & ( & k & (2) \\
\hline $0-110$ & & & 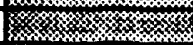 & . & $x_{2}$ & Xnt & 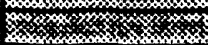 & 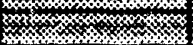 & 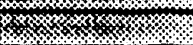 \\
\hline E-111 & & & (1) & 级, $1 \%$ & \% & 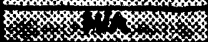 & (1) & \% & \%: \\
\hline E-112 & & & \% & 2.6 & 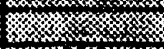 & $\%$ & & স & r. \\
\hline E-201 & & & 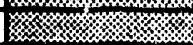 & & \% & ( & 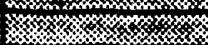 & . & ঋ \\
\hline E-202 & & & 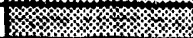 & স্যে- & 1. & ( & 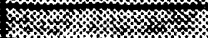 & . & 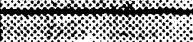 \\
\hline E-203 & & & 级 & ) & 流 & (1) & ( & 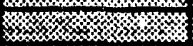 & স \\
\hline E-204 & & & $k$ & 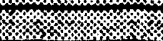 & (1; & L & 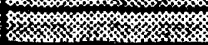 & . & শ. \\
\hline $6 x-101$ & & & oc & 淿 & 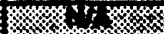 & | & ( & ( & \\
\hline$\overline{x-102}$ & $\bar{x}$ & & & צ' & 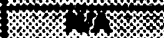 & t & 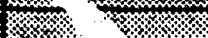 & 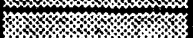 & \\
\hline Ex-103 & & & $\alpha c$ & 8 & / & , & 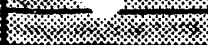 & ' & (1) \\
\hline$E x-104$ & & & कs & $x$ & $y_{1}$ & ty & r & 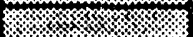 & \\
\hline $8 x-106$ & & & & W & 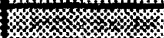 & 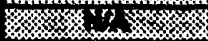 & Y & \% & \\
\hline $8 x-100$ & $\bar{x}$ & & & (2i) & 将 & $f \gamma$ & 4 & , & \\
\hline Bx-107 & & & OC & Wh: & $1 / 1.121 \%$ & F/ & & & \\
\hline
\end{tabular}

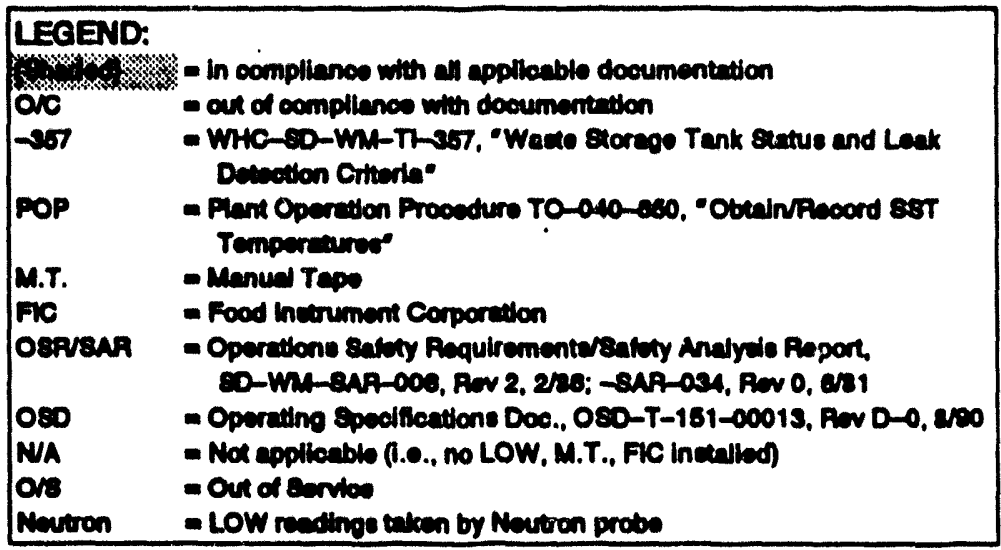


TABLE A-7. SINGLE-SHELL TANKS MONITORING COMPLIANCE STATUS 149 TANKS (Sheet 2 of 5)

Information as of May 31, 1994

\begin{tabular}{|c|c|c|c|c|c|c|c|c|c|}
\hline \multirow{4}{*}{$\begin{array}{l}\text { Tenk } \\
\text { Number }\end{array}$} & \multirow{2}{*}{\multicolumn{2}{|c|}{ Catecory }} & \multirow{4}{*}{$\begin{array}{c}\text { Temperature } \\
\text { Peadings } \\
(5)(6)\end{array}$} & \multirow{3}{*}{\multicolumn{2}{|c|}{$\begin{array}{l}\text { Surface Leval } \\
\text { Raadingos (1) } \\
\text { (-357) }\end{array}$}} & \multirow{4}{*}{$\begin{array}{c}\text { Low } \\
\text { Randinges } \\
(-357)(9) \\
\text { neution }\end{array}$} & \multicolumn{2}{|c|}{ Padlation Rasdings } & \multirow{4}{*}{$\begin{array}{l}\text { Dome } \\
\text { Eevation } \\
\text { survers } \\
\text { (OSPrSAP) }\end{array}$} \\
\hline & & & & & & & Lateral & Drywell & \\
\hline & \multirow{2}{*}{$\begin{array}{l}\text { Watch } \\
\text { Let(6) }\end{array}$} & \multirow{2}{*}{$\begin{array}{l}\text { High } \\
\text { Heat }\end{array}$} & & & & & Raedinges & Raedings & \\
\hline & & & & & FC & & $(-357)$ & (OSPVAR) & \\
\hline $4 x-100$ & & & 16. & & (1) & (1) & WW & & \\
\hline $8 x-100$ & & & orc & $2 \pi$ & & 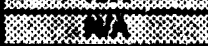 & 5 & & \\
\hline Ex-110(A) & & & 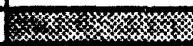 & (1) & X) & (1/ & XY) & \% & \% \\
\hline Ex-111(a) & & & . & & m. & & 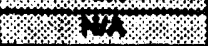 & & \\
\hline $\operatorname{ex-112}$ & & & אי & (2) & & W & SWV & & \\
\hline $8 Y-101(4)$ & & & & OKC & 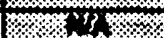 & $\gamma>$ & Wh & & 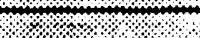 \\
\hline $8 Y-102$ & & & का & m & $6 \%$ & l' & $\mathrm{XSP}_{\mathrm{S}}$ & & \\
\hline $6 Y-103$ & $\bar{x}$ & & 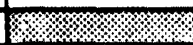 & ?. & ר. & & Wh & & \\
\hline$B Y-104$ & $\bar{x}$ & & ঋে & & \% & & W & & \\
\hline $8 Y-106$ & $\bar{x}$ & & 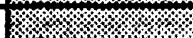 & & 3 & & W & & \\
\hline BY-100 & $\bar{x}$ & & & & 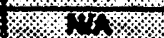 & & 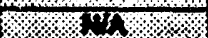 & & \\
\hline BY-107 & $\bar{x}$ & & 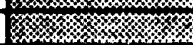 & צx & win & 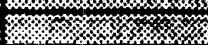 & 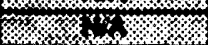 & & \\
\hline $6 Y-100$ & $\bar{x}$ & & 《x & & 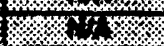 & \% & 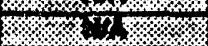 & x & 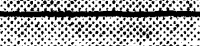 \\
\hline BY-100 & & & Or-ONC & 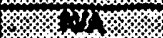 & & & 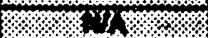 & $\$ \%$ & \\
\hline BY-110 & $\bar{x}$ & & 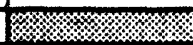 & & \%ors & & का & & \\
\hline $8 Y-111$ & $\bar{x}$ & & ? & & WW & & 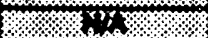 & & \\
\hline$B Y-112$ & $\bar{x}$ & & & & \%.n. & कc & w & $\%$ & \\
\hline $0-101$ & & & সে & & m & \%X? & $2 x$ & & \\
\hline$C-102$ & & & $o c$ & WWS & ?: & ?. "Vh & 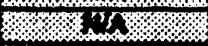 & ?.2M & \\
\hline$C-103$ & $\bar{x}$ & & & 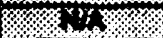 & : $:$ : & ר. & $x-1$ & খ & m \\
\hline$c-104$ & & & & WW: & & \%W & WW & থ: & ঋে: \\
\hline$C-106$ & & & & 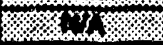 & ? & / 31 & 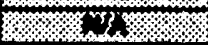 & প & 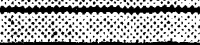 \\
\hline $0-100(A)$ & $\bar{x}$ & $\bar{x}$ & & $m$ & \% & (1) & $23 \%$ & ঝ / & \\
\hline $0-107$ & & & & $x$ & & $r y$ & $x$ & rে & \\
\hline$c-100$ & $\bar{X}$ & & \% & & (1) W & ক & 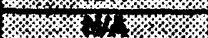 & אে & 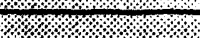 \\
\hline $0-100$ & $\bar{x}$ & & & & \%? & W W & r. $/ Y\}$ & পে & পে. \\
\hline$c-110$ & & & & & . & 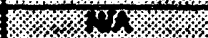 & . & V & \\
\hline$C-111$ & $\bar{x}$ & & & & ry & $7 y^{2}=4$ & 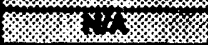 & $\forall>$ & খে \\
\hline C-112 & $\bar{x}$ & & 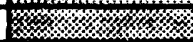 & $\%$ \% & \% $: 2 \%$ & 1. & 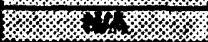 & 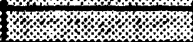 & (13: \\
\hline$C-201$ & & & $x$ & & 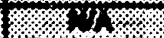 & V & 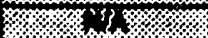 & ר & \% \\
\hline$c_{-202}$ & & & & 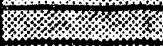 & \% & 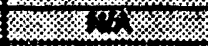 & Y & & ?. \\
\hline$c-203$ & & & 妶 & ঋ & m & r. & $\langle 4$ & rে & \\
\hline C-204 & & & oc & אי & $\%$ ris & \% & 3. & K & \\
\hline $8-101$ & & & & $x \%$ & . & $\eta_{7}$ & 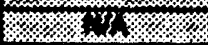 & থ & ? \\
\hline E-102 & $\bar{x}$ & & \% & $\sigma^{\prime}$ & \% & K/: & , & \% & \\
\hline $8-100$ & & & & 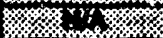 & / & $v_{1}$ & 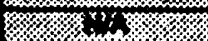 & 俩 & স্যে \\
\hline $2-104$ & & & s. & & 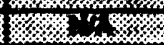 & k & k & א. & ২ে \\
\hline $2-106$ & & & & 43 & $y$ x & 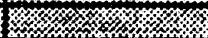 & 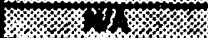 & 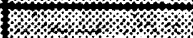 & \\
\hline-100 & & & 级 & 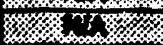 & 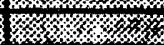 & k & $\gamma / \gamma^{\prime}$ & \% & (x) \\
\hline $8-107$ & & & & x. & $x_{x}^{\prime}$ & 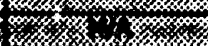 & Th & 将 & 文 \\
\hline$E-100$ & & & & 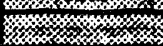 & 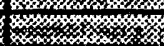 & 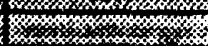 & (3) & (2. & X. \\
\hline $0-100$ & & & 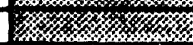 & \% & 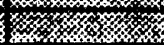 & $\gamma$ & 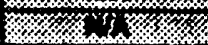 & \% & \\
\hline $8-110$ & & & & \% & 8 K & K & V & সে & 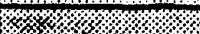 \\
\hline Q -111 & $\bar{X}$ & & 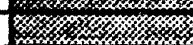 & 接 & 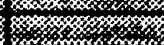 & 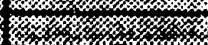 & $x^{\prime}$ & V & 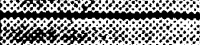 \\
\hline E-112 & $\bar{x}$ & & 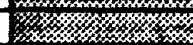 & $x_{2}$ & 8 & 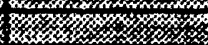 & $x_{2}$ & . & 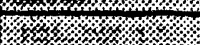 \\
\hline$E x-101$ & $\bar{x}$ & & 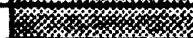 & s & $x_{k}$ & k & 媇 & U & र \\
\hline Ex-102 & $\bar{x}$ & & & $x$ & 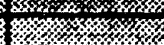 & 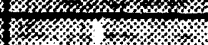 & $\frac{1}{x y 3}$ & & 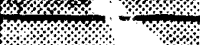 \\
\hline कx-10 & $\bar{x}$ & & & 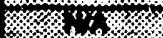 & S: : : : : & $t^{\prime}$ & 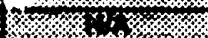 & 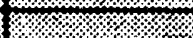 & m \\
\hline$\alpha x-104$ & $\ddot{x}$ & & স্যে & 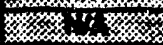 & 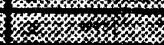 & $0=-0 C$ & 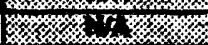 & / $/ 2 \%$ & \\
\hline$\alpha-100$ & $\bar{x}$ & & rা & 泣 & & & oc & $\eta_{2}$ & \\
\hline$\alpha-100$ & $\bar{x}$ & & (x) & 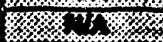 & 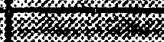 & $x_{1}^{\prime}$ & Thys & ( & V \\
\hline$x-107$ & & $\bar{x}$ & 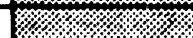 & & $z^{\prime} y, y$ & $\forall \times$ & oc & 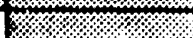 & $x^{\prime}$ \\
\hline $8 x-100$ & & & & & 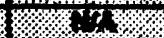 & The & क्ट & & \\
\hline
\end{tabular}


WHC-EP-0182-74

TABLE A-7. SINGLE-SHELL TANKS MONITORING COMPLIANCE STATUS 149 TANKS (Sheet 3 of 5 )

Information as of May 31, 1994

\begin{tabular}{|c|c|c|c|c|c|c|c|c|c|}
\hline \multirow{4}{*}{$\begin{array}{l}\text { Tank } \\
\text { Number }\end{array}$} & \multirow{2}{*}{\multicolumn{2}{|c|}{ Catcooy }} & \multirow{4}{*}{$\begin{array}{c}\text { Temperature } \\
\text { Rendinges } \\
\text { (5)(6) }\end{array}$} & \multirow{3}{*}{\multicolumn{2}{|c|}{$\begin{array}{l}\text { Surface Level } \\
\text { Reading (1) } \\
\text { (-357) }\end{array}$}} & \multirow{4}{*}{$\begin{array}{c}\text { LOW } \\
\text { Randinges } \\
(-357)(9) \\
\text { Neutron }\end{array}$} & \multicolumn{2}{|c|}{ Radiation Readinces } & \multirow{4}{*}{$\begin{array}{l}\text { Dome } \\
\text { Eevation } \\
\text { Surveys } \\
\text { (OSR/SAR) }\end{array}$} \\
\hline & & & & & & & \multirow{3}{*}{$\begin{array}{l}\text { Lateral } \\
\text { Rasdings } \\
(-357)\end{array}$} & \multirow{3}{*}{$\begin{array}{c}\text { Drywell } \\
\text { Resdings } \\
\text { (OSAYSAR) }\end{array}$} & \\
\hline & \multirow{2}{*}{$\begin{array}{l}\text { Watch } \\
\text { Lut(6) }\end{array}$} & \multirow{2}{*}{$\begin{array}{l}\text { High } \\
\text { Hext }\end{array}$} & & & & & & & \\
\hline & & & & M.T. & FIC & & & & \\
\hline $3-100(1)$ & $x$ & $x$ & 1) & & Why & 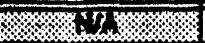 & OK & & \\
\hline$x-110$ & & $\bar{x}$ & 唼 & & YYS & $\mathrm{Y}_{\mathrm{SO}}$ & orc & & \\
\hline$x-111$ & & $\bar{x}$ & $x$ & & 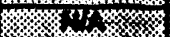 & 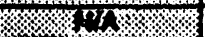 & orc & k & \\
\hline$x-112$ & & $\mathbf{X}$ & kx & \% & 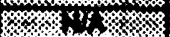 & 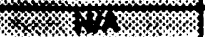 & ore & x & $1 \%$ \\
\hline C-ा13 & & & x & $\% 1 \%$ & $1 \times 1 \times 1 \%$ & 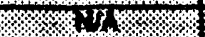 & 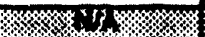 & $6 \%$. & $\%$ \\
\hline$\alpha-114$ & & $\bar{x}$ & x & \% & $\% 13$ & $\%$ W $\%$ & Ore & খ প & \\
\hline K-116 & & & ds-OC & & 81 & 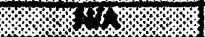 & Ore & & \\
\hline 1-101 (ब) & & & & & & 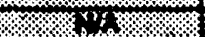 & $x \times$ & \% & \\
\hline$T-102$ & & & & 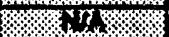 & OrC & (1) & 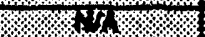 & & \\
\hline $7-103$ & & & m & 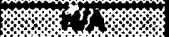 & & $81 \%$ & 43 & & \\
\hline $\mid-104$ & & & $O N$ & & $m$ & & $5 \pi$ & & $1 \%$ \\
\hline $1-100$ & & & OS-OK & 4 & m, & 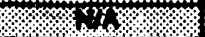 & 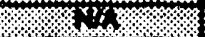 & & \\
\hline $1-100$ & & & & 83 & \% & $87 \%$ & \% 153 & $1 / .1 \%$ & \% \\
\hline T-107 & $\bar{x}$ & & 媇 & 83 & ২প & $\%$ W & ঋ" \%! & দৈ & ঋ/ \\
\hline$T-100$ & & & & & 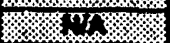 & 358 & 35 & $1 \%$ \% & $1 \%$ \\
\hline $1-100$ & & & oc & 83 & পফ & 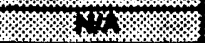 & $5 \%$ & & \\
\hline$T-110$ & $\bar{x}$ & & a & 8 & & & 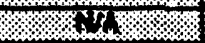 & & \\
\hline T-111 & $\bar{x}$ & & ঋে & WT: & 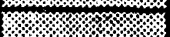 & & $\% ?$ & & \\
\hline F-112 & & & & $3 \pi$ & & $\pi M$ & $\pi /$ & & \\
\hline$T-201$ & & & צ & & $1 \% \%$ & 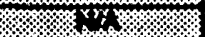 & $\mathrm{x}$ & & \\
\hline$P=202$ & & & & & 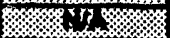 & 811 & 81 & & \\
\hline$T-203$ & & & & & 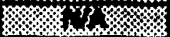 & 8318 & 8 & & \\
\hline$T-204$ & & & & & nst & ors & $\mathrm{BS}$ & & \\
\hline$x-101$ & & & $O B-O N C$ & $x \times 1$ & & $8 m$ & $8 \times 3 / \%$ & & \\
\hline $7 x-102$ & & & ON & & 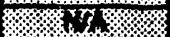 & & W1\% & & \\
\hline $7 x-105$ & & & xy & $\approx \%$ & & 814 & Y I & & \\
\hline ix-104 & & & \% & In: & & 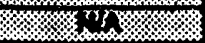 & 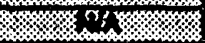 & \% & \\
\hline $7 x-106$ & $\bar{x}$ & & 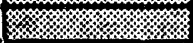 & 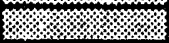 & 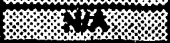 & ore-Orc & $2 \%$ & \% & \% \\
\hline $7 x-100$ & & & 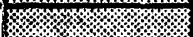 & & $3 \%$ & צ'প & W $Y \%$ & \% & ২ \\
\hline $7 x-107$ & & & ঋ & $\%$ & & $3 \%$ & $\%$ & & \\
\hline $7 x-100$ & & & ₹ & $8 n$ & & থ ' & $n^{n}, k x$ & $\%$ K & K $/ 1 \%$ \\
\hline $7 x-100$ & & & & $8)$ & & & $8 \mathrm{y}$ & পু & \\
\hline $7 x-110$ & & & ON & & $8 x$ & \% & 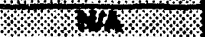 & & \\
\hline$\overline{T x-111}$ & & & ( & & $83 \%$ & & $Y$ & & \\
\hline Tx-112 & & & \% & \% / $/$. & 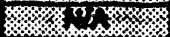 & & $3 \%$ & & \\
\hline $7 x-113$ & & & \% & 桨 & \% u' & xy & 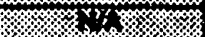 & & \\
\hline Tx-114 & & & OC & 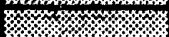 & $x y$ & & $8 \%$ & & \\
\hline $7 x-116$ & & & & & $1 \times 3 \%$ & $8 \%$ - & 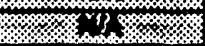 & \% & \% \\
\hline $7 x-110$ & & & $080 x$ & & W & 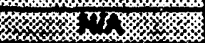 & $\gamma / \gamma^{n} / x$ & & \\
\hline $7 x-117$ & & & OC & & 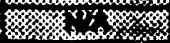 & zx & xym & & \\
\hline Tx-110 & $\bar{X}$ & & 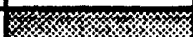 & $w^{\prime}$ & 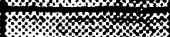 & $x^{\prime}$ & $x$ & $8 x$ & \\
\hline$\sqrt{-101}$ & $\bar{x}$ & & 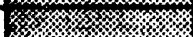 & $3 \%$ & $Y Y x x$ x & k & 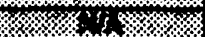 & & 翛 \\
\hline$\pi y-102$ & & & 络 & $1 \%$ & \% & x & KXY & & X \\
\hline$\pi-100$ & $\bar{X}$ & & ly & 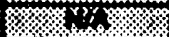 & 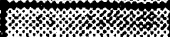 & 吆 & 3' & V. & $y x$ \\
\hline$n y-104$ & $\bar{x}$ & & 1\% & $\% \times 2 \%$ & 12.12 & 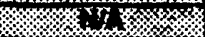 & $1 \times 1 \%$ & k & $\%$ \\
\hline rr-100 & & & W & \% & \% $3 x$ & x & 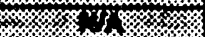 & প & X \\
\hline TY-100 & & & k & W $/ 2$ & 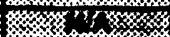 & $x^{\prime} x^{\prime}, x / x$ & 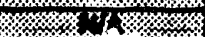 & $x$ & $\%$ \\
\hline U-101 & & & xys & & z & K & Kx & & 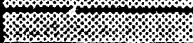 \\
\hline U102 & & & צr & $\left.16 y^{2}\right\}$ & k & 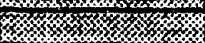 & 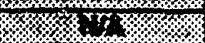 & & \\
\hline U-103 & $\bar{x}$ & & $x$ x & W & 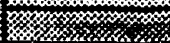 & 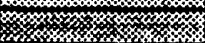 & y & \% & ঋy \\
\hline U-104 & & & $O / 8-O V C$ & 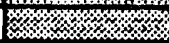 & \% & $x_{x} x_{x}$ & m & k & \\
\hline U-106 & $\bar{x}$ & & y & 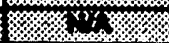 & s. & & $\%$ & & \\
\hline $0-100$ & $\bar{x}$ & & & 46 & 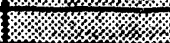 & & 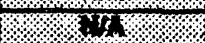 & $\%$ & \\
\hline
\end{tabular}




\section{TABLE A-7. SINGLE-SHELL TANKS MONTTORING COMPLIANCE STATUS 149 TANKS (Sheet 4 of 5)}

Information as of May 31, 1994

\begin{tabular}{|c|c|c|c|c|c|c|c|c|c|}
\hline \multirow{4}{*}{$\begin{array}{l}\text { Tank } \\
\text { Number }\end{array}$} & \multirow{2}{*}{\multicolumn{2}{|c|}{ Cencoory }} & \multirow{4}{*}{$\begin{array}{c}\text { Temperaturo } \\
\text { Resdings } \\
\text { (5)(6) }\end{array}$} & \multirow{3}{*}{\multicolumn{2}{|c|}{$\begin{array}{c}\text { Surface Level } \\
\text { Peading: (1) } \\
\text { (-357) }\end{array}$}} & \multirow{4}{*}{$\begin{array}{l}\text { LOW } \\
\text { Roadings } \\
(-357)(9) \\
\text { Nouron }\end{array}$} & \multicolumn{2}{|c|}{ Rodiation Poadings } & \multirow{4}{*}{$\begin{array}{l}\text { Dome } \\
\text { Eevation } \\
\text { Surveys } \\
\text { (OSPVSAR) }\end{array}$} \\
\hline & & & & & & & \multirow{3}{*}{$\begin{array}{l}\text { Leteral } \\
\text { Raading: } \\
\text { (-357) }\end{array}$} & \multirow{3}{*}{$\begin{array}{l}\text { Drymell } \\
\text { Readinas } \\
\text { (OSA/SAP) }\end{array}$} & \\
\hline & \multirow{2}{*}{$\begin{array}{l}\text { Watch } \\
\text { Let(6) }\end{array}$} & \multirow{2}{*}{$\begin{array}{l}\text { High } \\
\text { Heat }\end{array}$} & & & & & & & \\
\hline & & & & M.T. & FlC & & & & \\
\hline U-107 & $\bar{x}$ & & & XWX & \%" & & 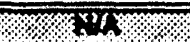 & & \\
\hline प-10 & $\bar{x}$ & & K & $x>$ & \% & \%焠 & $r x$ & & \\
\hline U-10 & $\bar{x}$ & & 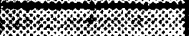 & 13 & K & $x$ ck & $3 \times 18$ & $\%$ & \\
\hline$U-110$ & & & א & $31 \%$ & & $\mathrm{mN}$ & तI & 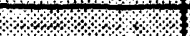 & \\
\hline U-111 & $\bar{x}$ & & צk & $\mathrm{W}$ & & & $x W$ & & \\
\hline U-112 & & & & & ren & $3 \times$ & In & & \\
\hline v-201 & & & & & 4 & Wy & Whe & & \\
\hline U-202 & & & & & IIS & 378 & 83 & & \\
\hline U-603 & & & & & 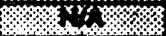 & $\mathrm{KW}$ & 46 & & $x$ \\
\hline U-204 & & & & & का & SYr & 213. & & \\
\hline \multicolumn{10}{|c|}{ Catch Tanks and Special Survellance Faciltiles } \\
\hline A. & NA & Nh & x & $\%: 3\}$ & & 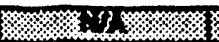 & $8: 3 \%$ & $63 \times$ & Y3. \\
\hline A.02-3 & NA & NA & 87 & & 131 & rots. & N. & 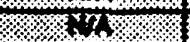 & 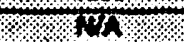 \\
\hline 311-EA & NA & N/A & 810\% & 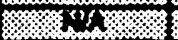 & & 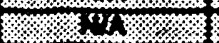 & $\%$ & $8 \times 3$ & 20 \\
\hline $162-\lambda x$ & NA & NA & rist & & Wh: & & & & \\
\hline $161-A 2$ & NA & WA & Wh & WOP & & WT & ary & Wh & nT: \\
\hline $15 \div-x_{2}$ & NA & WA & No & & nos & r & 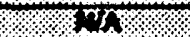 & m & Wh \\
\hline EX-TाJEAP & NA & NA & BTS & & WX & & & & \\
\hline A-TRESMP & WA & WA & $\sin$ & & 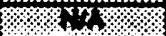 & \% & $8 / \%$ & $\%$ \% & . \\
\hline COARA & NA & W/A & 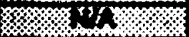 & & & & & & \\
\hline $417-A$ & NA & NA & 25 & & & & & & \\
\hline Vem Ean. & NA & WA & 83 & & 5 & (i) & 106 & 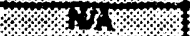 & $\mathrm{KM}$ \\
\hline 8002 & NA & NA & 3 & 5 & & 8 & W & WI & 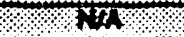 \\
\hline $802-A$ & NA & WA & $21 / 4$ & 31 & & $x y$ & 121 & Yor. & Tins: \\
\hline 820 & NA & WA & $2 y$ & & 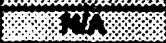 & ons & 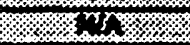 & $8 \%$ & Nh \\
\hline $1 x-02-8$ & NA & W/A & (1) & & $\%$ & 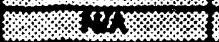 & \% 14 & 212 & 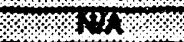 \\
\hline$\overline{x-302-c}$ & WA & WA & 8 & 5 & \% $/ 8$ & $2 \%$ & 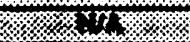 & $85 \%$ & IX \\
\hline U-01-9 & WA & NA & S'S & 5 & & \%? & 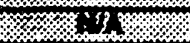 & 21 & 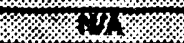 \\
\hline $0 x-502-n$ & NA & WA & Wx & rys & & 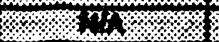 & $1 \%{ }^{n}-1$ & $8 \%$ & \% \\
\hline $141-6$ & NA & WA & 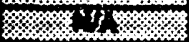 & & $n^{\prime}$, & 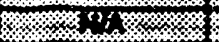 & 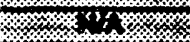 & - $\%$ ? $?$ & 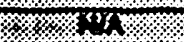 \\
\hline $142-8$ & NA & NA & Wr & & $2 x$ & $2 x$ & $876 \%$ & 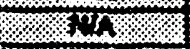 & W. \\
\hline $\begin{array}{l}\text { Totele: } \\
140 \text { tanke }\end{array}$ & $\begin{array}{c}40 \\
\text { Weath } \\
\text { Uit } \\
\text { Tenks } \\
\text { (4) }\end{array}$ & $\begin{array}{l}10 \\
\text { Hidon } \\
\text { Hent } \\
\text { Tents }\end{array}$ & $\begin{array}{l}\text { Orc: } \\
20 \text { tanke - } \\
\text { (momiannual } \\
\text { moniloring } \\
\text { moqueney) (5) }\end{array}$ & $\begin{array}{l}\text { Orc: } \\
1 \text { tank } \\
0 \text { centch teniks }\end{array}$ & $\begin{array}{l}\text { Or: } \\
1 \text { tank } \\
0 \text { cetch untos }\end{array}$ & 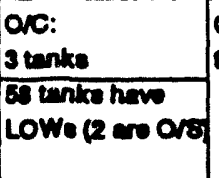 & $\begin{array}{l}\text { OrC: } \\
\text { otanks }\end{array}$ & 0 & 0 \\
\hline
\end{tabular}

\section{See Footnotes on next page}




\section{TABLE A-7. SINGLE-SHELL TANKS MONITORING COMPLIANCE STATUS 149 TANKS (Sheet 5 of 5 )}

\section{Footnotes:}

1. All SST have either manul tepe or FIC, with the exception of S-108 and T-101, which have both. Tenk 101-T lso has zip cord. All ssi FiCs are comected to cass, with the exception of BX-106; houver, the conraction for many tanks is broken. For such cases, manual readings ore taken. Manulal surface level readings include readings taken by manual tare, manulal FIC (not connected to CASS; 8x-106), manual reedings of outomatic FIC (if Cass is printing "0"), or eutconatic FIC. In som cases, the surfece lavel reedings are taken using a zip cord. While less eccurate, such readings are acceptable for meeting the surface level reading requirements.

2. Wigh heat tenks hove ective exhausters; peychrometrics are taken in these tanks (A-104/105, c-105/106 [effective July 1, 1993, C-105 is no langer a high heat land tank], 5X-107, 108, 109, 110, 111, 112, and 114). The exhausters on A-104/105 have been doun since october 1991; no readings are being taken. Psychrometric readings have not been taken in the sx high heat laad tanks since July 1993. The frequency of paychrametric readings in SSTs is deterwined by the Cognizant Engineers for the applicable tank farm on an Mas needed' basis, "with the exception of tanks C-105/106. Hanford Foderal Facility Agreeanent and Consent Order," Mashington State Department of Ecology, U. S. Environmental Protection Agency, and U. S. Department of Fieroy," 1992 (Tri-Party Agreement) requires peychrometric readings to be taken in C-105/106 on a monthly frequency. Peychrometric resdings were taken in May 1994.

3. In-tank photographs ore requested on an was needed" basis. Mo in-tank photographs were taken between Septenber 1990 and March 1993.

4. Two tanks are on both category lists (C-106 and 5X-109). In July 1993, C-105 was reavoved from the Wigh Heat Load list and BX-110, BX-111, BY-101 and T-101 were removed from the ferrocyanide Watch List; these tanks continue to have temperature readings taken meekly al though they are only required to be taken semiarmull ly.

5. Temperature readings my be regulated by OSD, -357 , or POP. Additionally, high heat load tenks are regulated by OSR/SAR. Thermocouples in the nine tonks designated O/S-O/C ore out of service; there ore either no thermocouple trees in these tanks, or trees have been cut off, covered over, or are otherwise unable to function. The OSD does not require reedings or repair of out-of service thernocouples for the 91 low heat losd $(440,000 \mathrm{Btw} / \mathrm{h})$ tanks. However, the POP requires that at tempts ore to be made semi armually in Jenuary and July to obtain reedings for these tanks. Temperatures were taken in January 1994; total of 20 tanks are $0 / C$ because readings could not be obtoinad.

6. "Sefety Measures for Waste Tanks at Henford Muclear Reservation, Section 3137 of the Mational Defense Authorizotion Act for Fiscal Year 1991, "Novenber 5, 1990, Public Law 101-510, (the muyden Amendment(") requires continuous pressure monitoring and temperature monitoring in Watch List tanks. WHC-EP-0422 REV 1, "A Plan to Implement Rewediation of Waste Tank Safety Issues at the Hanford site," December 1991, addresses these monitoring issuss. WHC-EP-0600, "status Report on Resolution of Haste Tenk Sofety Issues at the Henford Site," issued August 1993, describes the resolution strategy for these safety issues.

- All BY-Farm tanks on the ferrocyenide watch list are on the Tank Monitor and Control System (TMACS) which contimously anitors for temperatures. In addition, all of C-Farm was added March 31, 1994. with the exception of C-204, wich has no thermocouple tree.

7. Contimuas Air Monitoring (CAM) complience and Redietion Ares Monitorino Panel (RAP) compliance ore not eddreseed in this table.

8. Double-shell tenk farm sy has the only tenks with continuous vapor/flasmable gas monitoring; not addreased in this table. 


\section{TABLE A-8. DOUBLE-SHELL TANKS MONTTORING COMPLIANCE STATUS 28 TANKS (Sheet 1 of 2 )}

The following table indicates whether Double-Shell tank monitoring was in compliance with the requirements as specified in the applicable documents as of the last day of the applicable month:

NOTE:

Dome Eovition Eurvoys ere not requlred tor DSTs. Poyehrometrice (2)

In-tank Photographs (3)

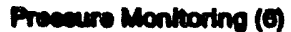

CAMARMAP Monttoring (7)

Vapor Monitoring (8)

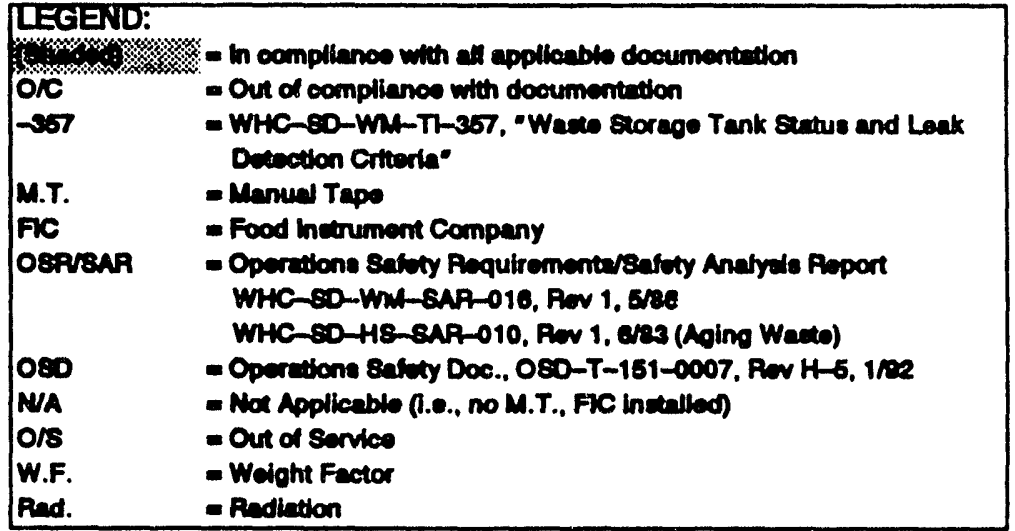

Information as of May 31, 1994

\begin{tabular}{|c|c|c|c|c|c|c|c|}
\hline \multirow{3}{*}{$\begin{array}{l}\text { Tank } \\
\text { Number }\end{array}$} & \multirow[b]{3}{*}{ Watch List } & \multirow{3}{*}{$\begin{array}{c}\text { Temperature } \\
\text { Readings } \\
\text { (4) } \\
\text { (OSD) }\end{array}$} & \multirow{2}{*}{\multicolumn{2}{|c|}{$\begin{array}{l}\text { Surface Level } \\
\text { Readings (1) } \\
\text {-357, OSRYSAP) }\end{array}$}} & \multicolumn{3}{|c|}{ Radiation Readinos } \\
\hline & & & & & \multicolumn{2}{|c|}{$\begin{array}{c}\text { Leak Detection } \\
\text { Ptts (5) } \\
(-357, \text { OSP/SAP) }\end{array}$} & \multirow{2}{*}{$\begin{array}{c}\text { Annulus } \\
(-357)\end{array}$} \\
\hline & & & M.T. & FIC & W.F. & Rad. & \\
\hline AN-101 & & & $\%$ & & 聯 & r. & \\
\hline AN-102 & & & 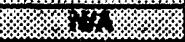 & & & \% & \\
\hline AN-103 & $\bar{x}$ & & $8 \%$ & & & & \\
\hline NN-104 & $\bar{x}$ & & 5 & & & $6 / 2$ & 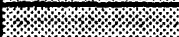 \\
\hline AN-105 & $x$ & & 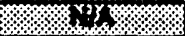 & & & & \\
\hline A. 100 & & & 68 & & & 2 & \\
\hline At-107 & & & 2 & \% & \% & $\%$ & \\
\hline AP-101 & & ك̌ & 得 / ₹ & 㧒䛑 & . & OC & \\
\hline AP-102 & & 媇媇奴 & k & ors & 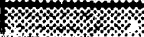 & ore & 㶴 \\
\hline Ap-103 & & 纹 & & 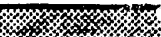 & భ & OX & $\%$ \\
\hline Ap-104 & & 森聯 & Ors & & x & Or & c c c c \\
\hline AP-106 & & & (1.: & ors & $\%$ \% 21 & Ore & xy \\
\hline AP-100 & & & \%k & צxy & x & orc & \\
\hline NP-107 & & 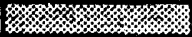 & 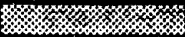 & 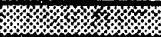 & 2\% & oc & \% \\
\hline N2-108 & & & & & & कx & k \\
\hline$A \sqrt{W-101}$ & $\bar{x}$ & & X䋞 & . & & 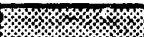 & \\
\hline AW-102 & & & & $\gamma / x$ c & c & x & \\
\hline$A W-103$ & & 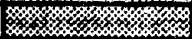 & 䋇 & 好 & 絲 & 짔 & z \\
\hline$A W-104$ & & 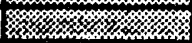 & 经 & 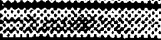 & 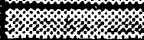 & 媇 & 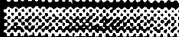 \\
\hline$A W-106$ & & 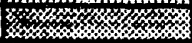 & 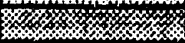 & xk & x & Ore & 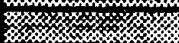 \\
\hline$A W-100$ & & som & sxy & $x$ x s & 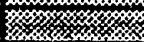 & 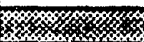 & 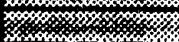 \\
\hline AY-101 & & 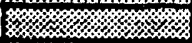 & \% & ov & 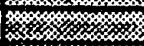 & s. & ONC \\
\hline AY-102 & & 嬨 & x & 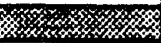 & 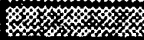 & Oc & कe \\
\hline$A z-101$ & & 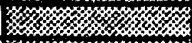 & 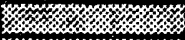 & ors & 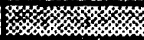 & 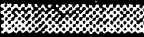 & 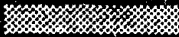 \\
\hline 12-102 & & 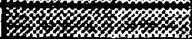 & 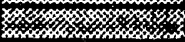 & 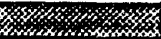 & 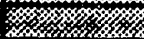 & Ore & 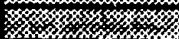 \\
\hline vy-901 & $\bar{x}$ & 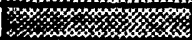 & 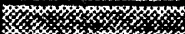 & x & 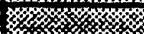 & & 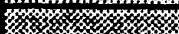 \\
\hline$y-102$ & & 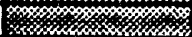 & m & 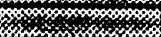 & x & . & 绽 \\
\hline $87-103$ & $\bar{x}$ & 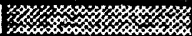 & 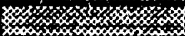 & 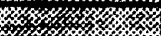 & 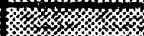 & сй & \\
\hline $\begin{array}{l}\text { Totaler } \\
\text { eas tanks }\end{array}$ & Watch Lin Tenks & or: & Onc: & $\begin{array}{l}\text { Or: } \\
0\end{array}$ & $\begin{array}{l}\text { orc: } \\
0\end{array}$ & $\begin{array}{l}\text { Orc: } \\
11 \text { indere }\end{array}$ & $\begin{array}{l}\text { Orc: } \\
2 \text { tanks }\end{array}$ \\
\hline
\end{tabular}

Sea footnotes next page: 
TABLE A-8. DOUBLE-SHELL TANKS MONITORING COMPLIANCE STATUS 28 TANKS (Sheet 2 of 2)

\section{Footnotes:}

1. All DSTs hove both manual tape and FIC, with the exception of the AN Tank Farm which has only FICs. The eanual tape is wed then the FIC is out of service. O/C will be shown when no readings are abtoined.

2. Peychrometric readings are only taken on tanks with active exhausters; all DSTs have active exhausters. The frequency of peychrometric rasdings in OSTs is determined by the Cognizant Enoinuers for the apolicable tank farmo on en "as needed" basis. Currently, monthly readings are being taken on the sY-101 anmulus exhaust, sy-102 tank and ammilus exhaust, and sY-103 tank and amulus exhaust. SY-101 tank exhaust reedings are not being taken until a port on the tank exhoust heeder becomes ovailable for exhauster readings. No other paychrometric readings are currently being taken monthly.

3. In-tank photographs are requested on an was needed" basie. List in-tank photographs in DSTs were taken in April 1989.

4. OSD specifies DST temperature limits, gradients, etc. Tank SY-101 temperatures are obtained shiftwise with increased readings taken prior to and following gas venting.

5. Failure of both leak detection systems requires repair of at least one system within 5 working days. Failure of one system only, repair must be within 10 workdays per -357 document. If the pair of out-of-service system exceeds these timeframes, all systems are 0/C. Out-of-service systems which have not exceeded these timefranes will be shoin as O/S.

6. " Mafety Measures for Waste Tanks at Hanford Muclear Reservation, Section 3137 of the Mational Defense Authorization Act for Fiscal Year 1991, "November 5, 1990, Publ ic Law 101-510, (the "uyden Amendment") requires continuous pressure monitóring and temperature monitoring in Watch List tanks. UHC-EP-0422 REV 1. WA Plan to Implement Remediation of Waste Tank Safety lssues ot the Henford site." Deceuber 1991, addresses these monitoring issues. A status report on resolution of Haste Tank Safety issues ot the Manford Site has been prepared but has not yet been cleared for public release.

7. Continuous Air Monitoring (CAM) compliance and Radiation Area Monitoring Panel (RAMP) compliance ore not addressed in this table.

8. Double-shell tenk farm SY has the only tanks with continuous vapor/flemable gas monitoring; not codreseed in this table. 
TABLE A-9. AUTOMATIC FOOD INSTRUMENT CORPORATION (FIC) GAUGES OUT OF SERVICE

May 31, 1994

Date of Last

Automatic

Tank No. SST/OST FIC Reading

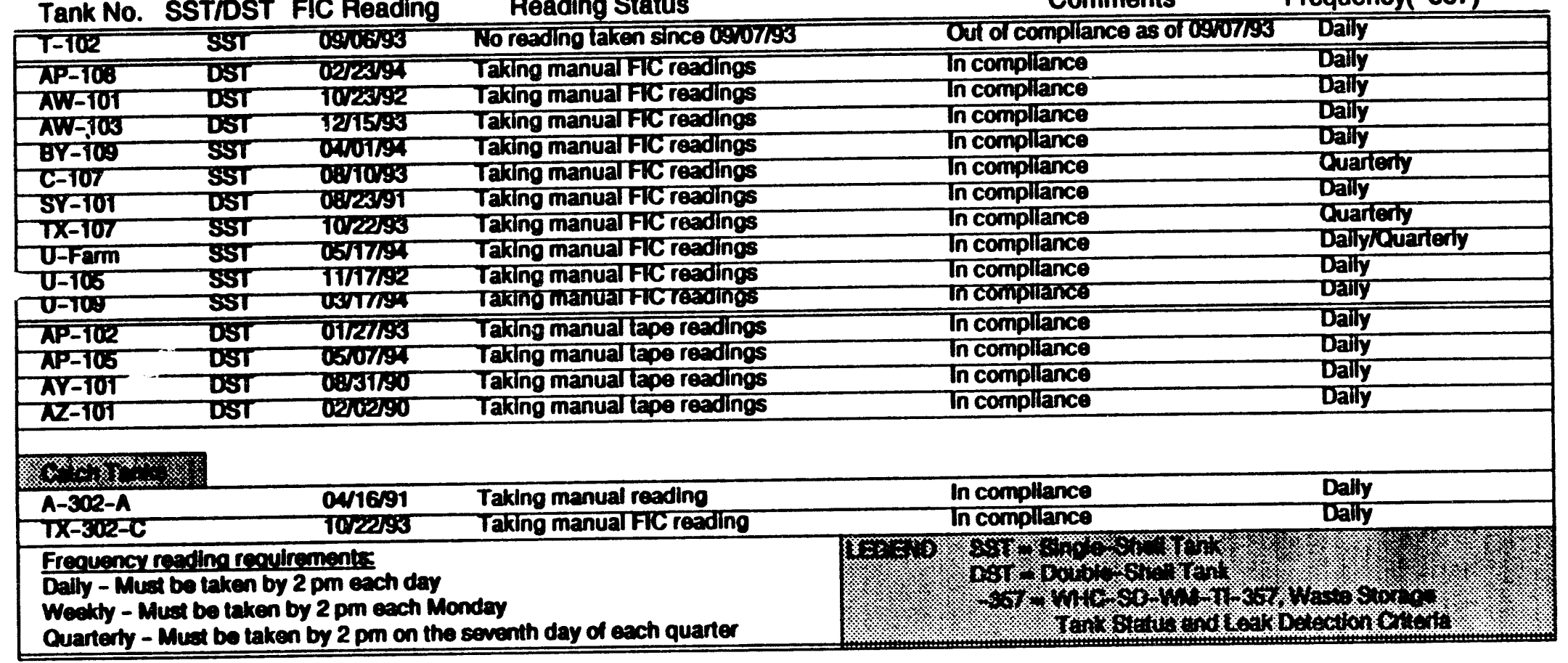




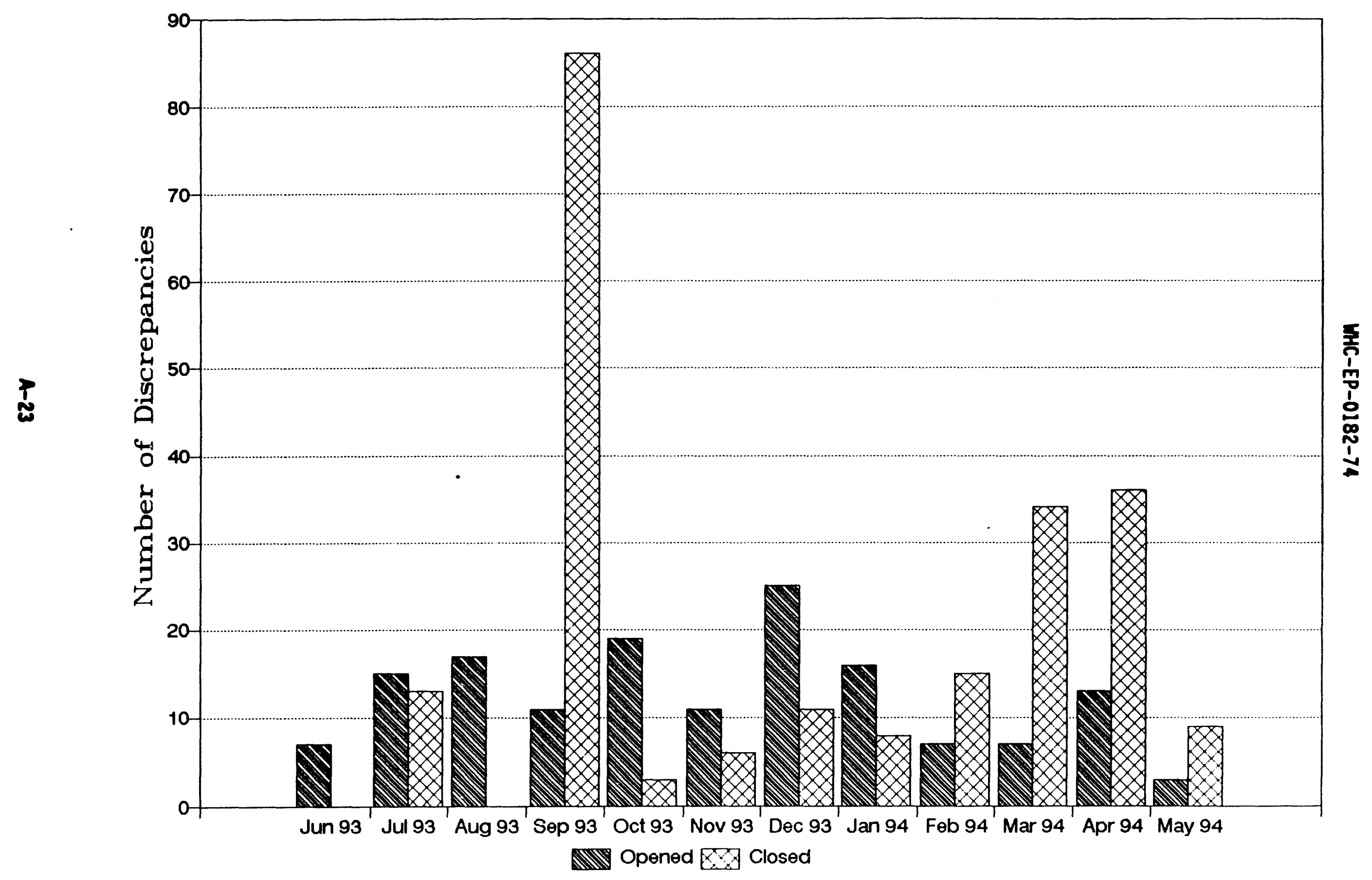

Figure 1. Discrepancy Report Status 


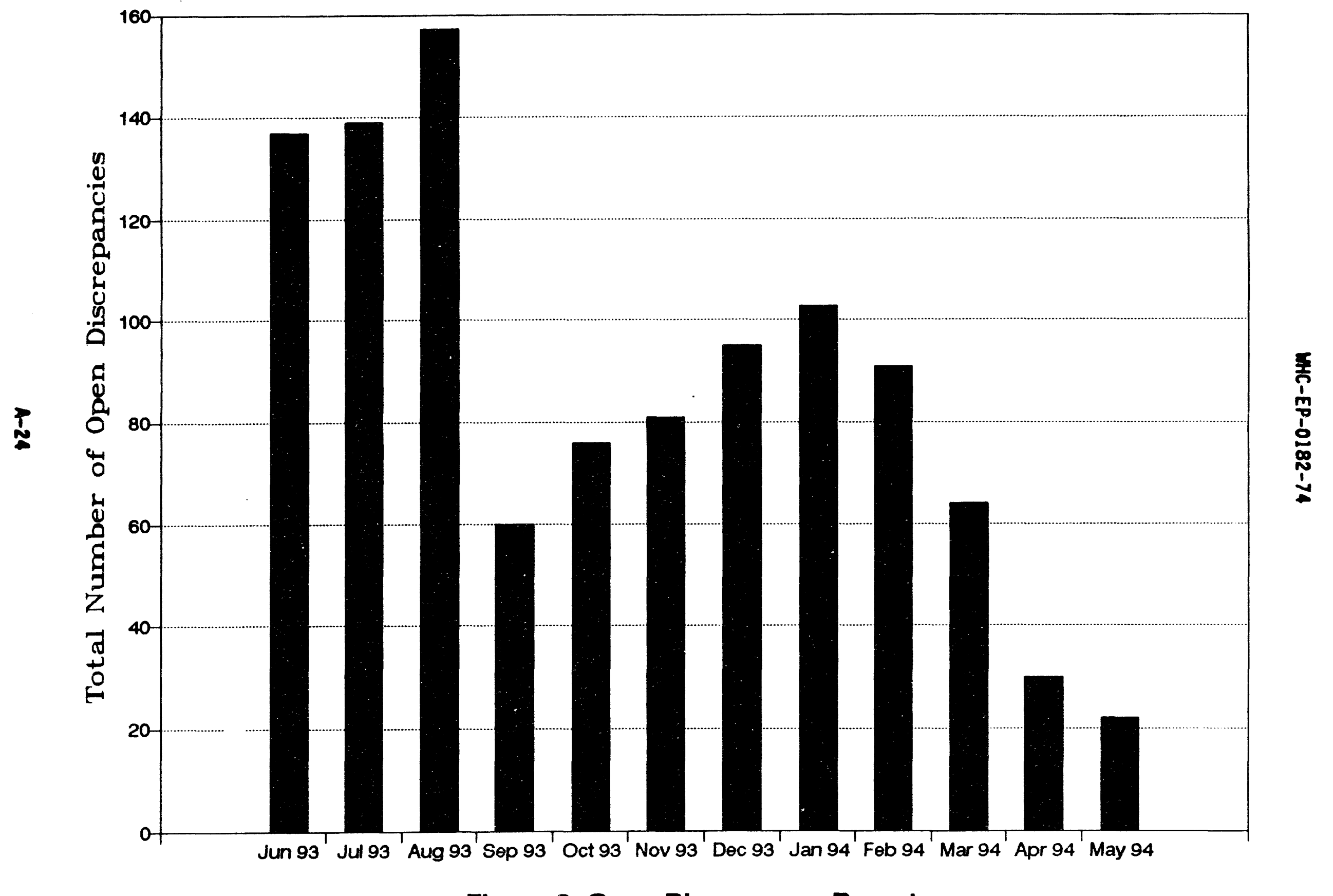

Figure 2. Open Discrepancy Reports 


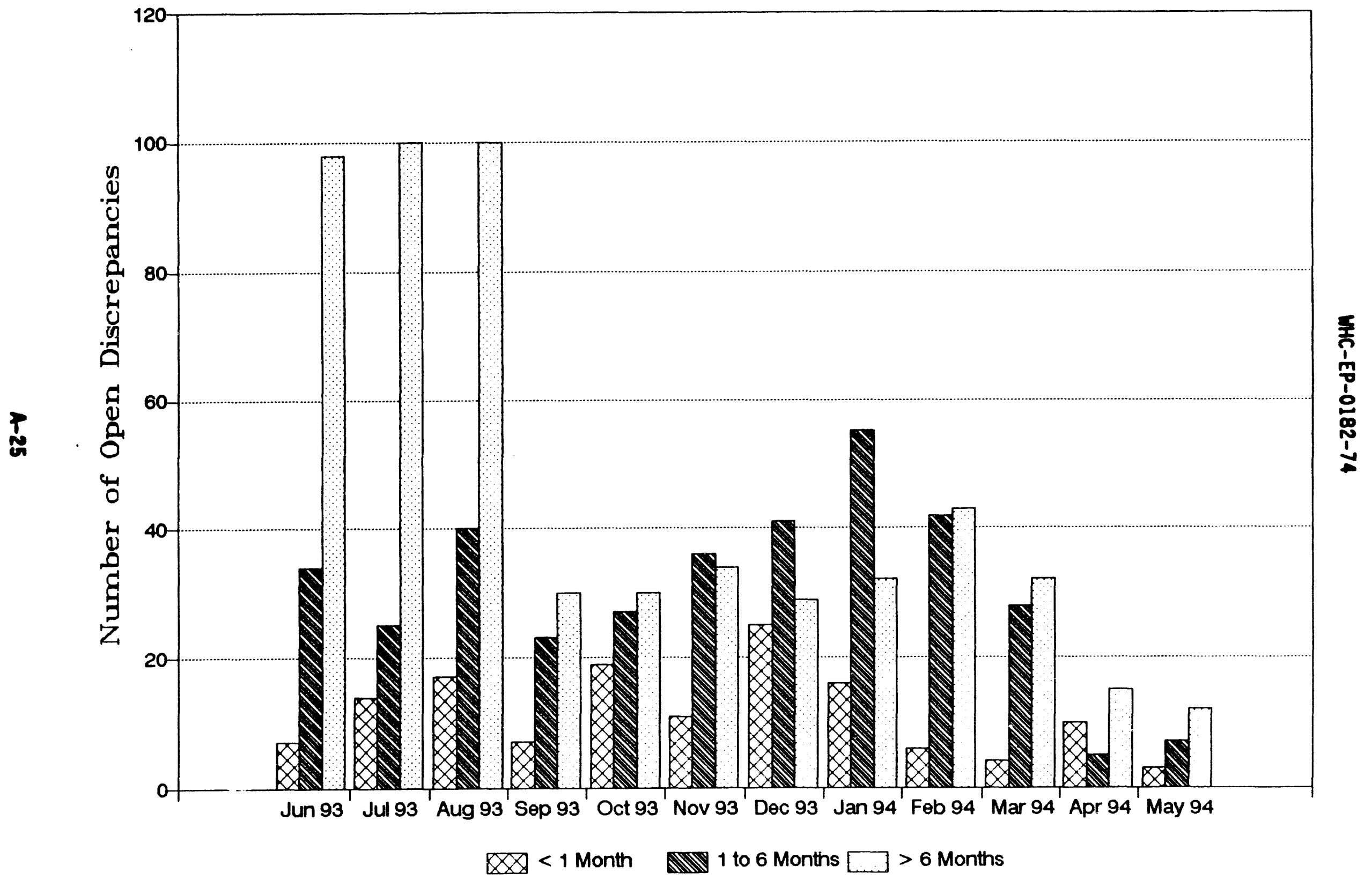

Figure 3. Discrepancy Report Status by Age 
MHC-EP-0182-74

This page intentionally left blank 
WHC-EP-0182-74

\section{APPENDIX B}

\section{DOUBLE SHELL TANK WASTE TYPE} AND SPACE ALLOCATION 
WHC-EP-0182-74

This page intentionally left blank 
TABLE B-1. DOUBLE-SHELL TANK WASTE TYPE AND SPACE ALLOCATION MAY 1994

DOUBLE-SHELL TANK INVENTORY BY WASTE TYPE SPACE DESIGNATED FOR SPECIFIC USE

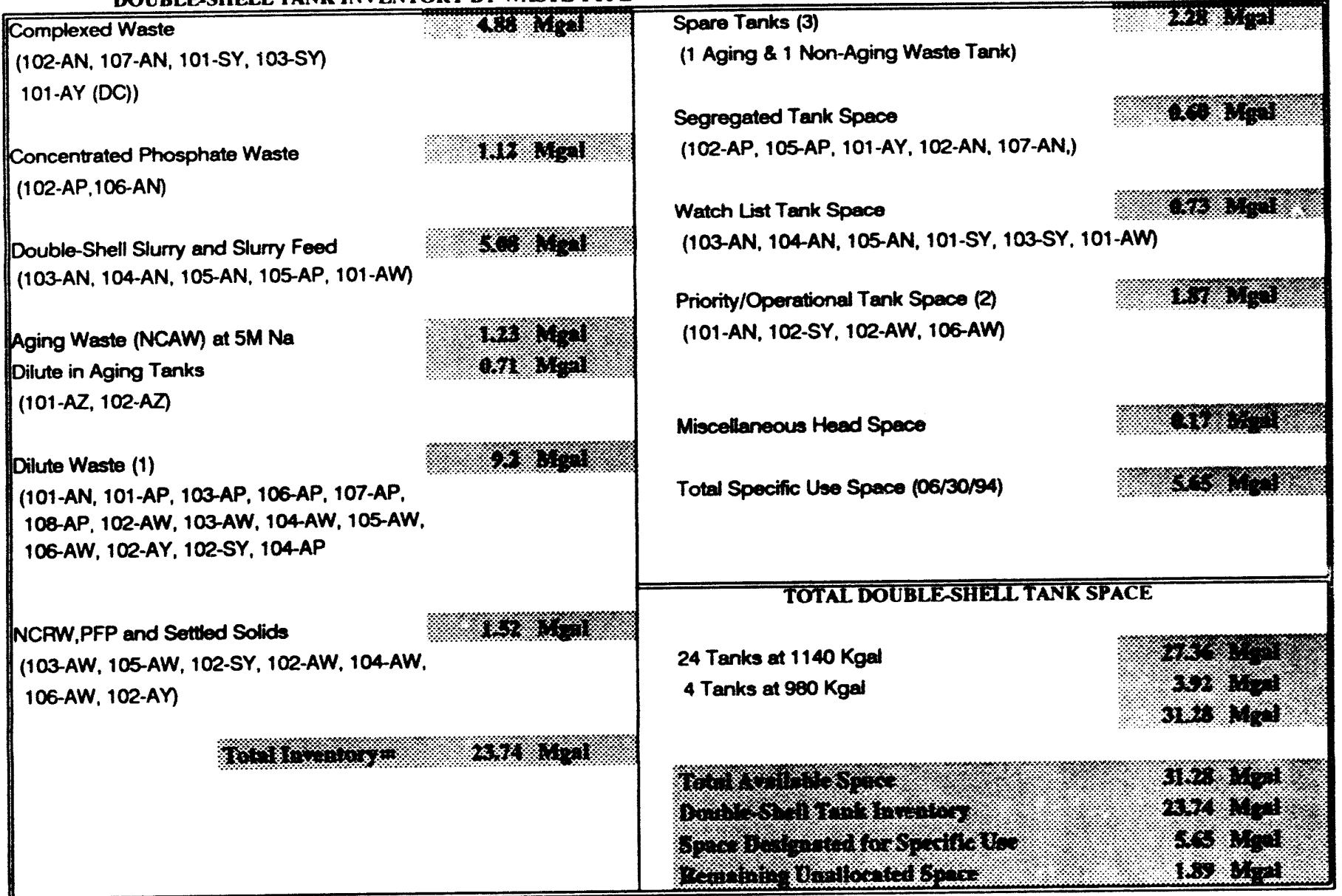

(1) Was reduced in volume by -1.397 Mgal this month (Evaporator Waste Volume Reduction)

(2) Reduced by Saltwell Liquid pumping, and PFP Operations

(3) 241-101-AY: A minumum liquid level is set to provide extra protection against any bottom uplitting of the tank's steel iirer. WHC-SO-WM-Th-357.

Wa

Waste Slorage Tank Status and Leak Detection Criteria," specifies 64 in. as the minimum surface level meesurememt when the ansultion systom is in operation, and 18 in. if the annulus ventilation system is shut down. See also OSD-T-151-0007. "Unclassified Operation Specifications for the 241 AN, 241AP, 241 AW, 241AY and 241SY Tank Farms." Because of space availablitity, waste is stored in 102-AY, the aging waste spare tank. Ih case of a leak, the contents of 102-AY will be distributed to any other DST(s) having available space. Tank 104-AP has been designated as the non-aging spare tank. 


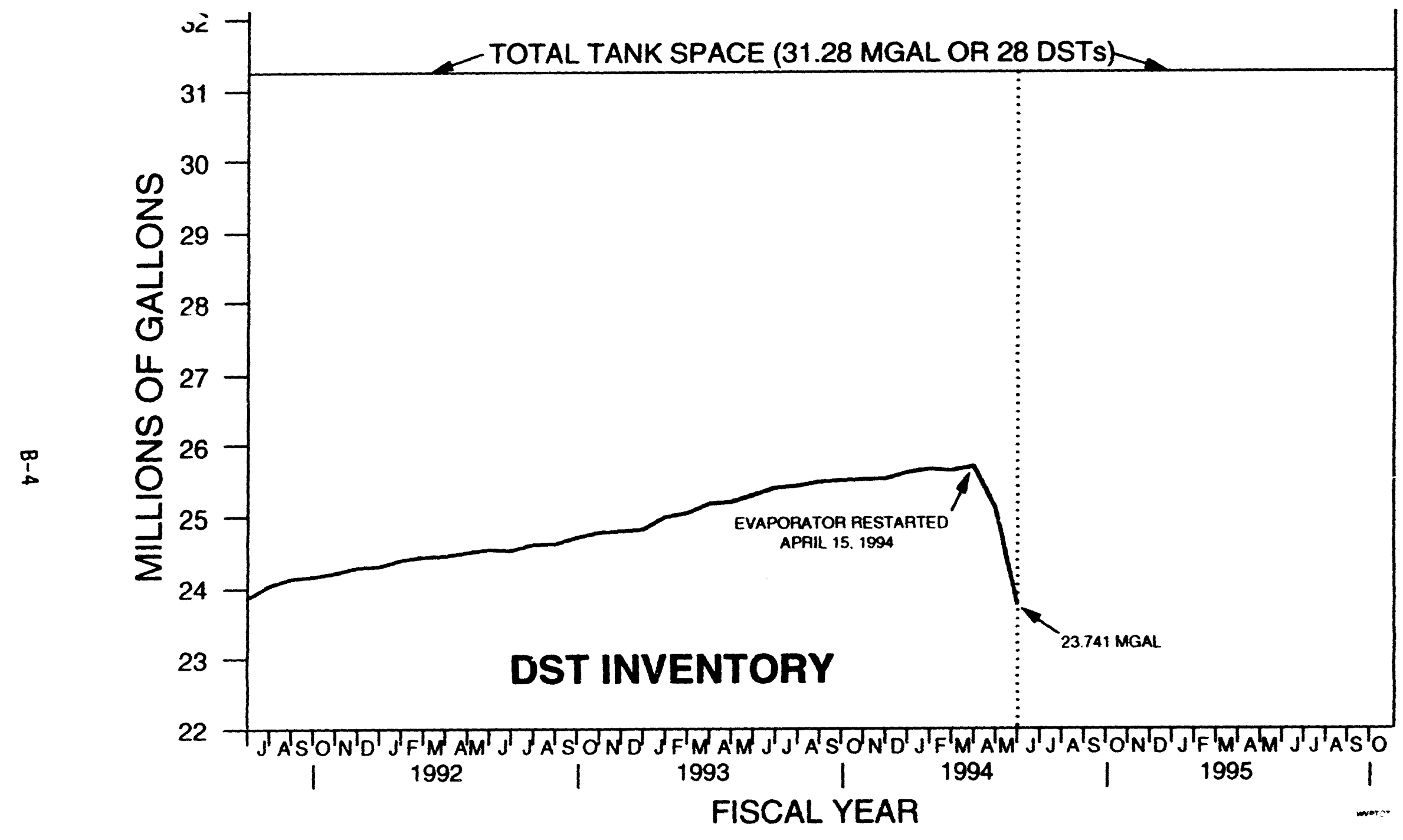

FIGURE B-1. TOTAL DOUBLE-SHELL TANK INVENTORY AND CHANGES 


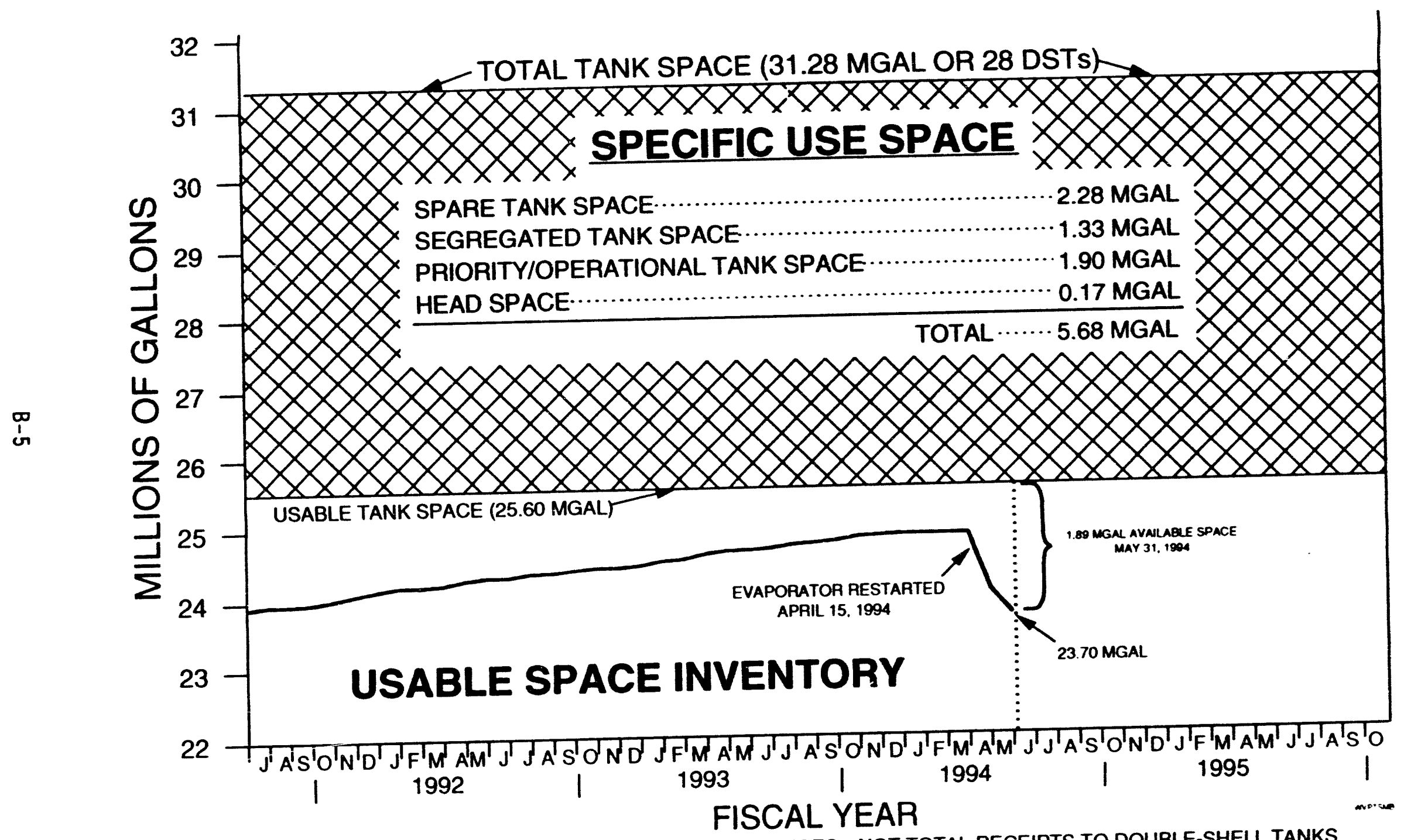




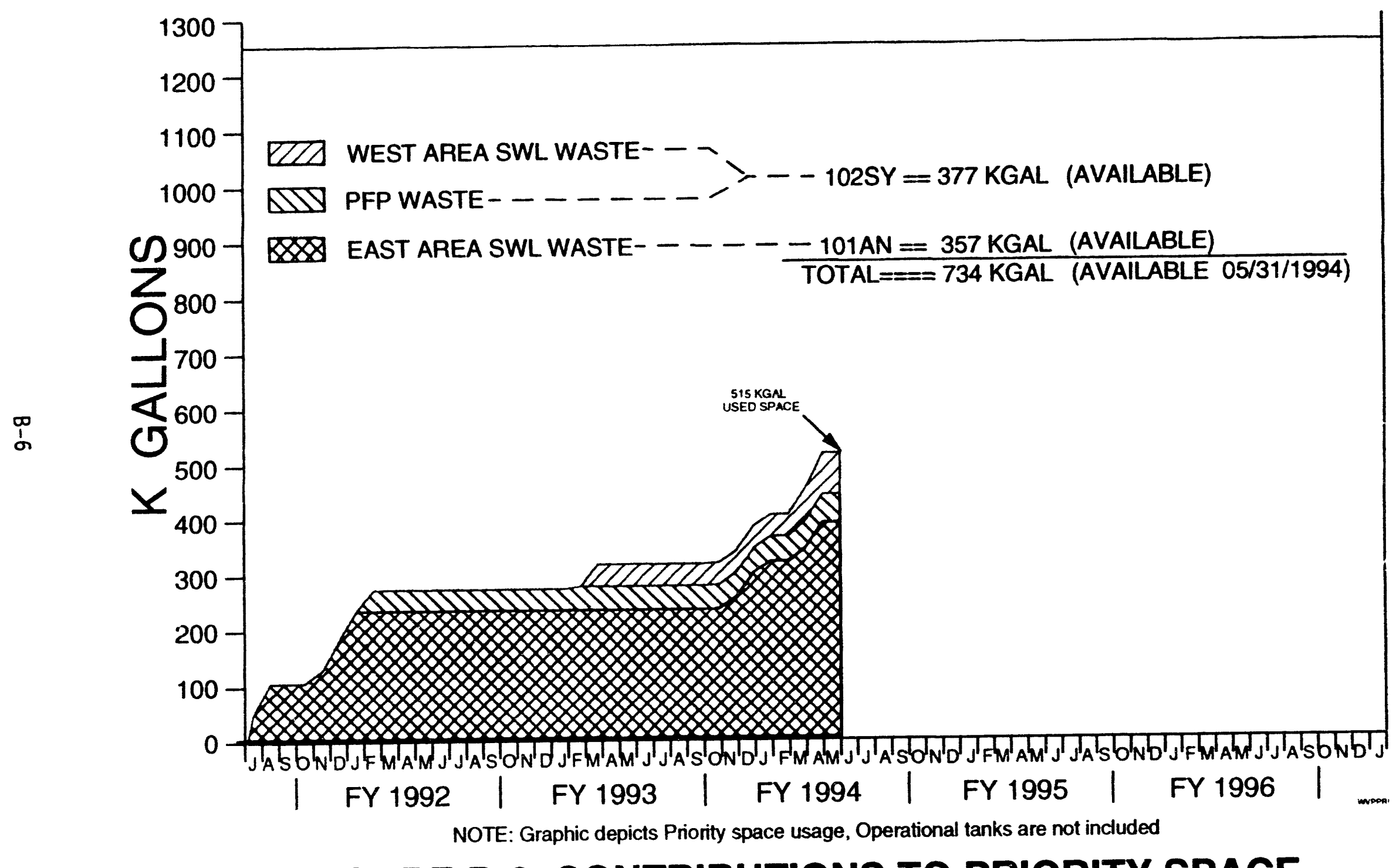

\section{FIGURE B-3. CONTRIBUTIONS TO PRIORITY SPACE}




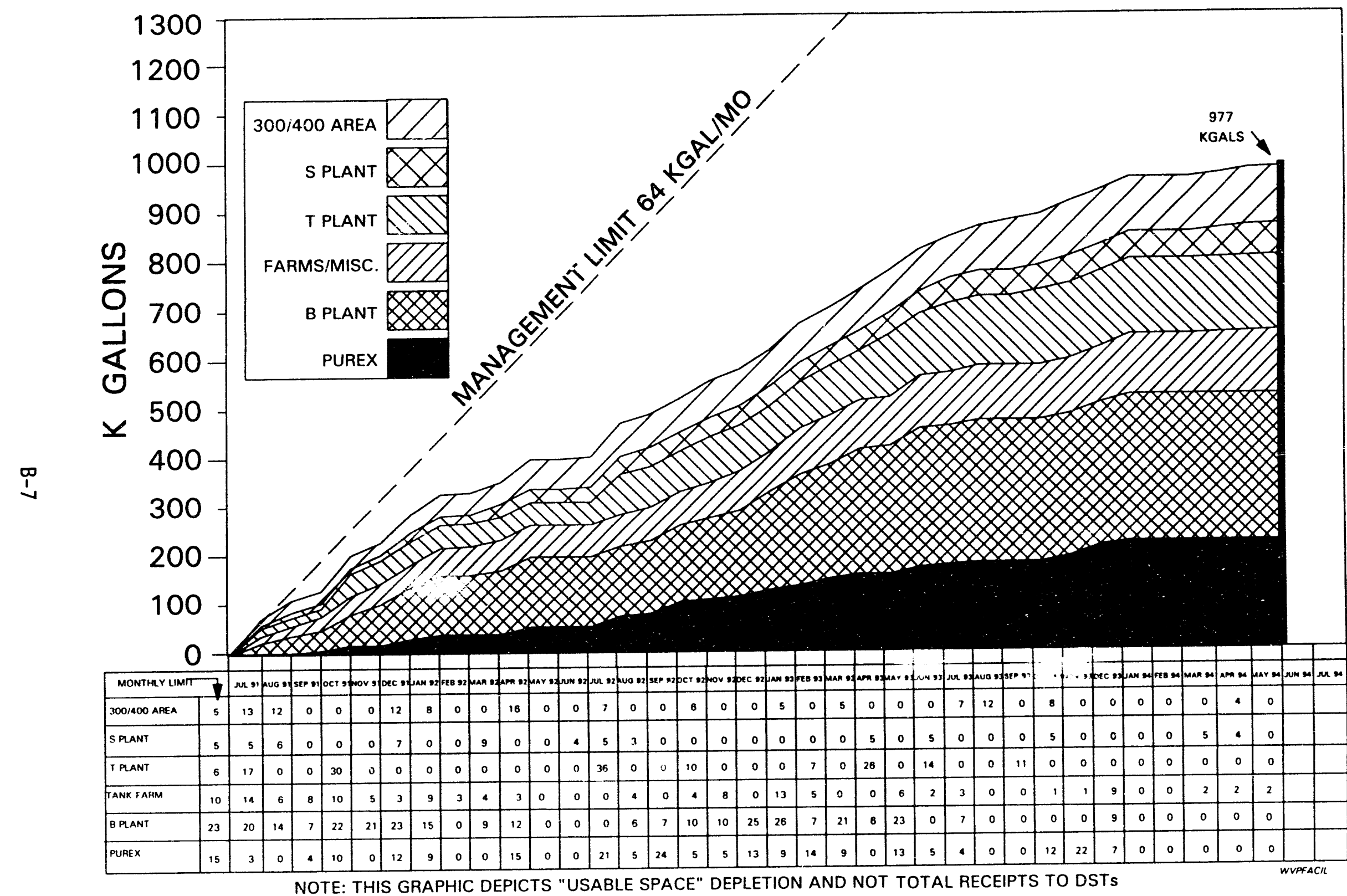

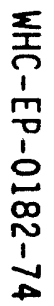
FIGURE B-4. COMPARISON OF FACILITY GENERATATIONS TO MANAGEMENT LIMIT 


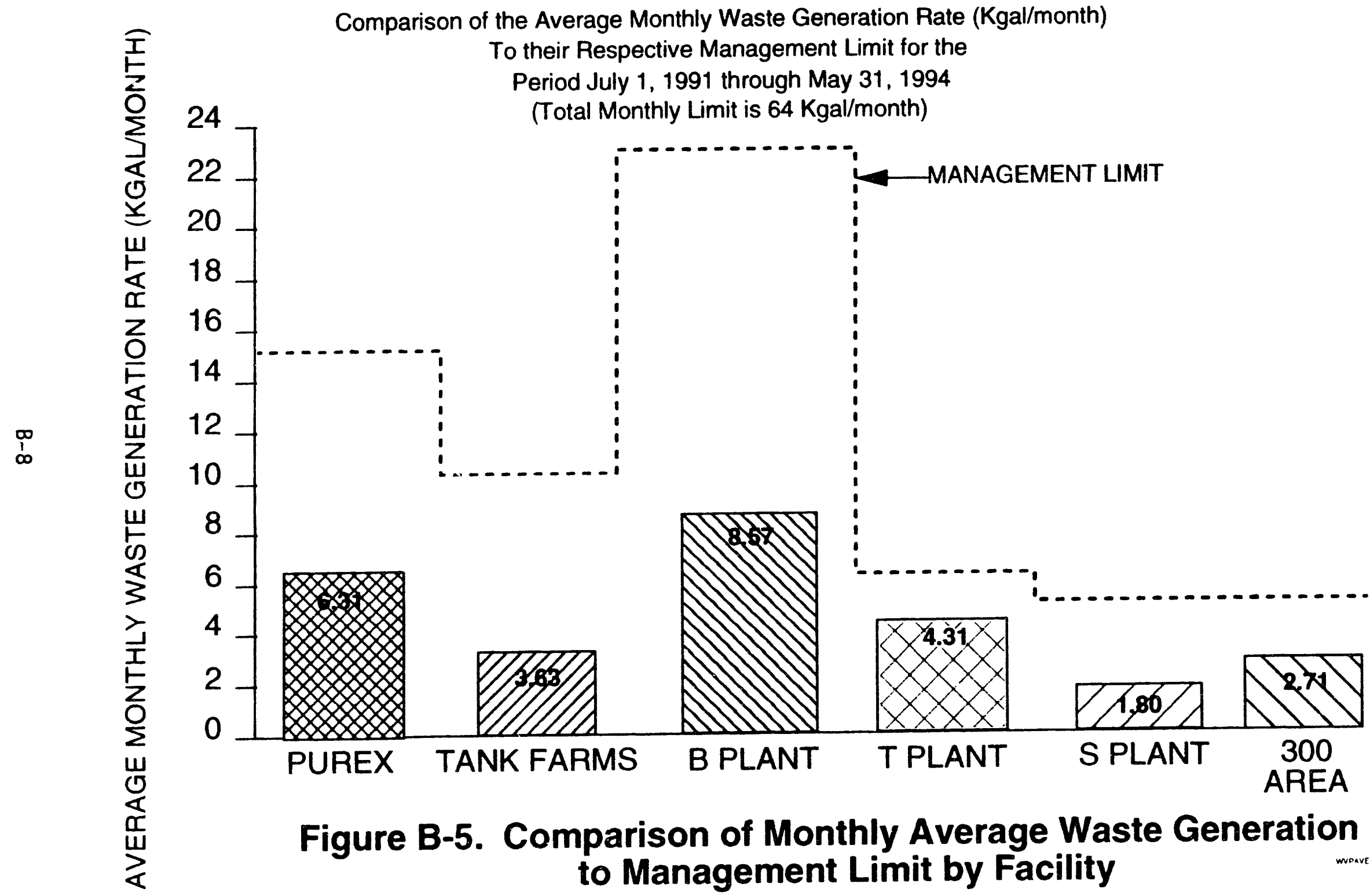




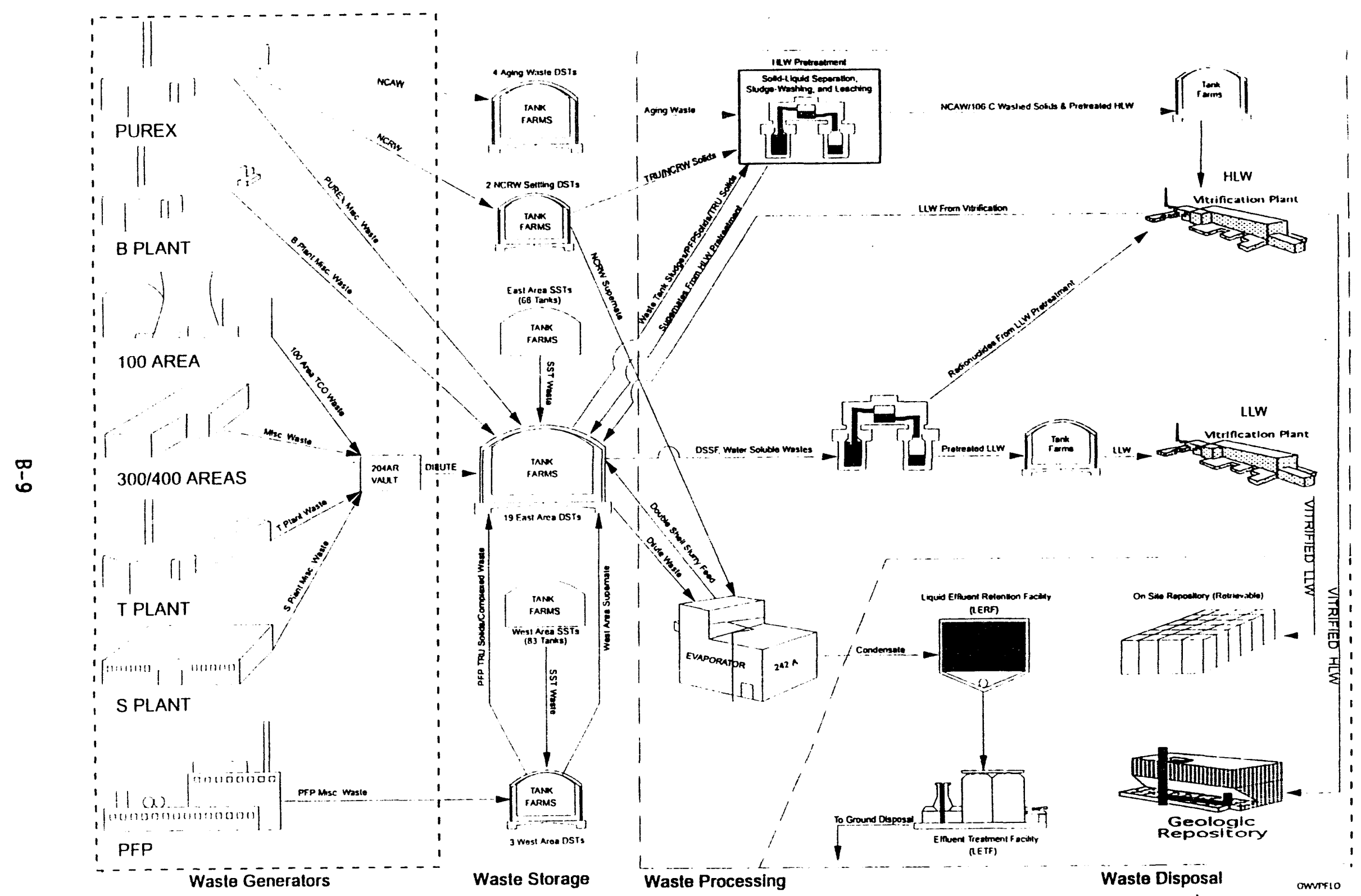

Figure B-6. Overall Waste Flow - Hanford Tank Waste Disposal 
Table B-2. Double Shell Tank Waste Inventory for May 31, 1994

(page 1 of 2)

\begin{tabular}{|c|c|c|c|c|}
\hline TANKS & INVENTORY & SOLIDS & TYPE & LEFT \\
\hline $101 \mathrm{AW}=$ & 1124 & 84 & DSSF & 16 \\
\hline $102 \mathrm{AW}=$ & 215 & 3 & DN & 925 \\
\hline $103 \mathrm{AW}=$ & 647 & 487 & NCRW & 493 \\
\hline $104 \mathrm{AW}=$ & 1123 & 267 & DN & 17 \\
\hline $105 \mathrm{AW}=$ & 1043 & 388 & NCRW & 97 \\
\hline $106 A W=$ & 964 & 211 & DN & 176 \\
\hline $101 \mathrm{AY}=$ & 877 & 83 & DC & 103 \\
\hline $102 A Y=$ & 719 & 32 & DN & 261 \\
\hline ho1AZ= & 967 & 35 & NCAW & 13 \\
\hline $102 A Z=$ & 967 & 95 & NCAW & 13 \\
\hline $101 \mathrm{AN}=$ & 783 & 0 & DN & 357 \\
\hline $102 A N=$ & 1088 & 89 & $\mathrm{CC}$ & 52 \\
\hline $103 \mathrm{AN}=$ & 953 & 373 & DSS & 187 \\
\hline $104 A N=$ & 1057 & 264 & DSSF & 83 \\
\hline $105 \mathrm{AN}=$ & 1124 & 0 & DSSF & 16 \\
\hline $106 \mathrm{AN}=$ & 21 & 17 & $C P$ & 1119 \\
\hline $107 \mathrm{AN}=$ & 1062 & 134 & CC & 78 \\
\hline $1015 Y=$ & 1103 & 560 & CC & 37 \\
\hline hO2SY $=$ & 763 & 133 & PT/DN & 377 \\
\hline $1035 Y=$ & 748 & 4 & CC & 392 \\
\hline IO1AP $=$ & 1060 & 0 & DN & 80 \\
\hline $102 \mathrm{AP}=$ & 1102 & 0 & $C P$ & 38 \\
\hline $103 A P=$ & 28 & 0 & DN & 1112 \\
\hline HOAAP $=$ & 18 & 0 & DN & 1122 \\
\hline HO5AP $=$ & 817 & 0 & DSSF & 323 \\
\hline $106 \mathrm{AP}=$ & 1127 & 0 & DN & 13 \\
\hline 107AP $=$ & 1110 & 0 & DN & 30 \\
\hline HOBAP $=$ & 1131 & 0 & DN & 9 \\
\hline $10 \%$ & $8 \times 14$ & & 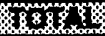 & \% \\
\hline
\end{tabular}

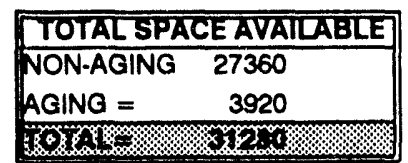

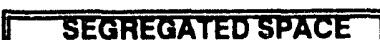

\begin{tabular}{|lr|}
\hline $101 A W=$ & 16 \\
$102 A P=$ & 38 \\
$105 A P=$ & 323 \\
$101 S Y=$ & 37 \\
$1035 Y=$ & 392 \\
$101 A Y=$ & 103 \\
$102 A N=$ & 52 \\
$103 A N=$ & 187 \\
$104 A N=$ & 83 \\
$105 A N=$ & 16 \\
107AN $=$ & 78 \\
\hline WATCHLIST TANKS
\end{tabular}

\begin{tabular}{|ll|}
\hline \multicolumn{3}{|c|}{ MISC. HEADSPACE } \\
\hline 101AP $=$ & 80 \\
106AP $=$ & 13 \\
107AP $=$ & 30 \\
104AW $=$ & 17 \\
101AZ $=$ & 13 \\
102AZ $=$ & 13 \\
\hline OS & \\
\hline
\end{tabular}

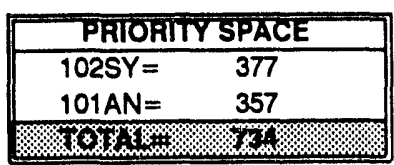

\begin{tabular}{|c|c|}
\hline \multicolumn{2}{|c|}{ TNVENTORY CHANGE } \\
\hline 04/94 TOTAL & 25121 \\
\hline 05/94 TOTAL & 23741 \\
\hline TENTESTS & 101 \\
\hline
\end{tabular}

\begin{tabular}{|c|c|}
\hline \multicolumn{2}{|c|}{ USABLE SPACE } \\
\hline $103 A P=$ & 1112 \\
\hline $104 \mathrm{AP}=$ & 1122 \\
\hline $108 \mathrm{AP}=$ & 9 \\
\hline 103AW = & 493 \\
\hline $105 \mathrm{AW}=$ & 97 \\
\hline $102 A Y=$ & 261 \\
\hline $106 \mathrm{AN}=$ & 1119 \\
\hline $102 A W=$ & 925 \\
\hline $106 \mathrm{AW}=$ & 176 \\
\hline Cors & 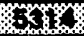 \\
\hline SPARES & -2280 \\
\hline EVAP. OPERATION & -1140 \\
\hline 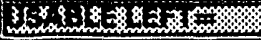 & $8 \&$ \\
\hline
\end{tabular}

USABLE SPACE CHANGE 04/94 TOTAL SPACE 1131 05/94 TOTAL SPACE 1894 L

PRIORIT 04/94 TOTAL SPACE 05/94 TOTAL SPACE Tis
Inventory Calculation by Waste Type:

\begin{tabular}{|c|c|c|}
\hline \multicolumn{3}{|c|}{ COMPLEXED WASTE } \\
\hline $102 A N=$ & 1088 & (CC) \\
\hline $107 \mathrm{AN}=$ & 1062 & (CC) \\
\hline $1015 Y=$ & 1103 & (CC \& DSS) \\
\hline ho3sY= & 748 & (CC, DSS \& SWL) \\
\hline $101 A Y=$ & 877 & (DC) \\
\hline SIX & 20 & \\
\hline \multicolumn{3}{|c|}{ NCAW SOLIDS (PD) } \\
\hline $103 \mathrm{AW}=$ & 487 & \\
\hline $105 \mathrm{AW}=$ & 388 & \\
\hline OXX & $\mathrm{WL}$ & \\
\hline
\end{tabular}

\begin{tabular}{|l|l|}
\hline PFP SOL!DS (PT) \\
\hline TO2SY $=$ & 133 \\
\hline TOSPY
\end{tabular}

\begin{tabular}{|c|c|}
\hline \multicolumn{2}{|c|}{ CONCENTRATED PHOSPHATE (CP) } \\
\hline $106 \mathrm{AN}=$ & 21 \\
\hline HO2AP $=$ & 1102 \\
\hline 18.1\% & 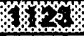 \\
\hline
\end{tabular}

\begin{tabular}{|c|c|}
\hline \multicolumn{2}{|c|}{ DILUTE WASTE (DN) } \\
\hline $101 \mathrm{AP}=$ & 1060 \\
\hline $103 \mathrm{AP}=$ & 28 \\
\hline $106 \mathrm{AP}=$ & 1127 \\
\hline $107 \mathrm{AP}=$ & 1110 \\
\hline $108 \mathrm{AP}=$ & 1131 \\
\hline $101 \mathrm{AN}=$ & 783 \\
\hline $102 A W=$ & 212 \\
\hline $104 A W=$ & 856 \\
\hline $106 \mathrm{AW}=$ & 753 \\
\hline $102 A Y=$ & 687 \\
\hline $104 A P=$ & 18 \\
\hline $103 \mathrm{AW}=$ & 160 \\
\hline $105 \mathrm{AW}=$ & 655 \\
\hline $102 S Y=$ & 630 \\
\hline $60 \%$ s & 240 \\
\hline \multicolumn{2}{|c|}{$\begin{array}{l}\text { NCAW (AGING WASTE } \\
\text { (@ } 5 \mathrm{M} \mathrm{Na)}\end{array}$} \\
\hline $101 \mathrm{AZ}=$ & 791 \\
\hline $102 A Z=$ & 434 \\
\hline $8 \times 3 \%$ & 485 \\
\hline $\mathbf{s u}=1$. & 800 \\
\hline TOKX & oss. \\
\hline
\end{tabular}

B-10

\begin{tabular}{|lr|}
\hline & DSSTDSSF \\
\hline $105 A P=$ & 817 \\
$103 A N=$ & 953 \\
$104 A N=$ & 1057 \\
$105 A N=$ & 1124 \\
$101 \mathrm{AW}=$ & 1124 \\
\hline BST & 504 \\
\hline
\end{tabular}

\begin{tabular}{|lr|}
\hline \multicolumn{2}{|c|}{ GRANDTOTALS } \\
\hline CC $=$ & 4001 \\
DC $=$ & 877 \\
NCRW SOLIDS $=$ & 875 \\
DST SOLIDS $=$ & 513 \\
PFP SOLIDS $=$ & 133 \\
CP $=$ & 1123 \\
NCAW $=$ & 1934 \\
DSS/DSSF $=$ & 5075 \\
DILUTE $=$ & 9210 \\
OS WW \\
\hline
\end{tabular}

NOTE: All Values are in Kilogallons. (*) Watch List Tanks 
WHC-EP-0182-74

Table B-2. Double Shell Tank Waste Inventory for May 31, 1994

(page 2 of 2)

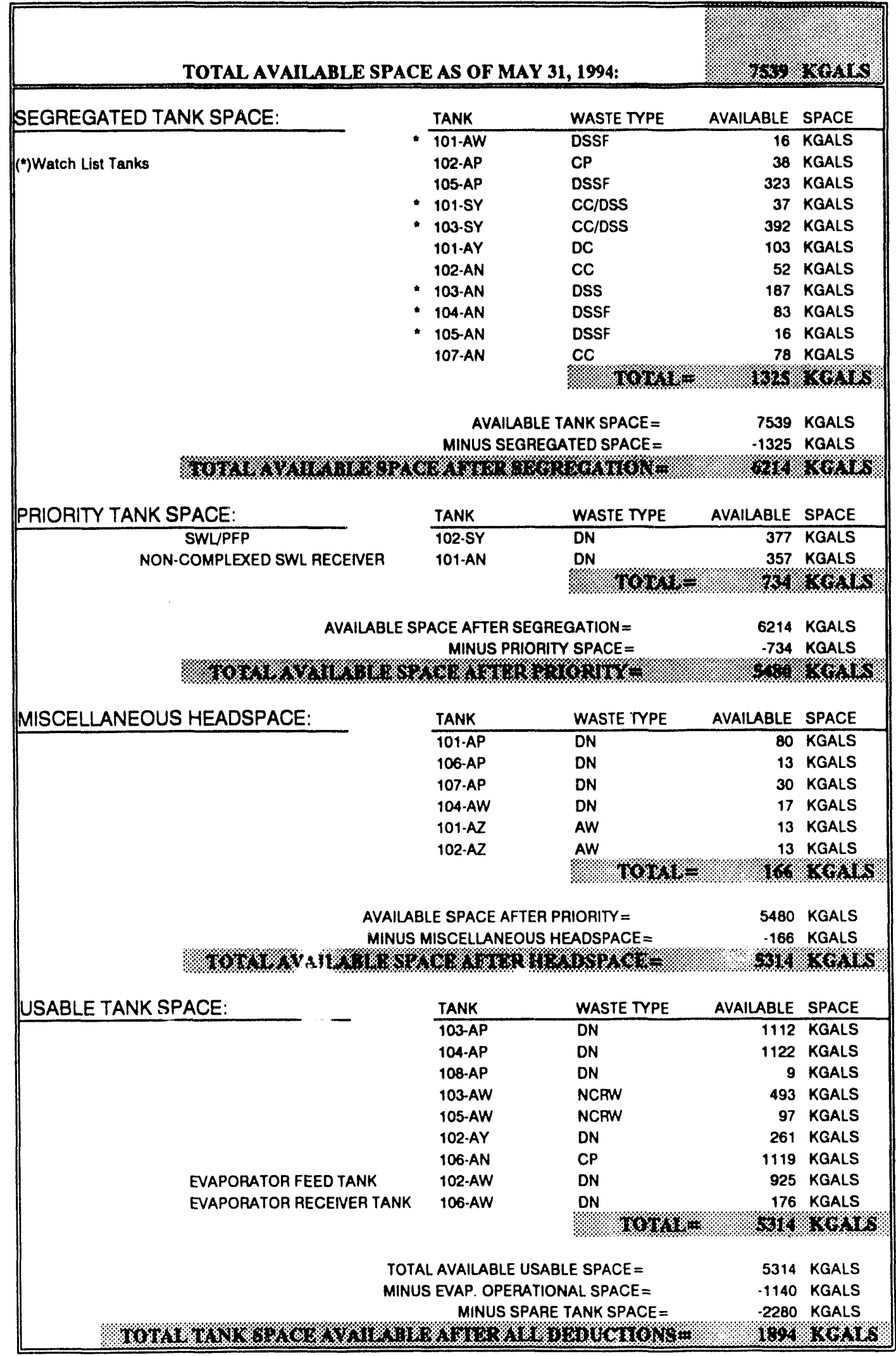




\section{WHC-EP-0182-74}

This page intentionally left blank 
WHC-EP-0182-74

\section{APPENDIX C}

TANK AND EQUIPMENT CODE AND STATUS DEFINITIONS 
WHC-EP-0182-74

This page intentionally left blank 
WHC-EP-0182-74

\section{TANK AND EQUIPMENT CODE/STATUS DEFINITIONS \\ May 31, 1994}

\section{TANK STATUS CODES}

\section{WASTE TYPE}

$\begin{array}{ll}\text { AGING } & \text { Aging Waste (Neutralized Current Acid Waste [NCAW]) } \\ \text { CC } & \text { Complexant Concentrate Waste } \\ \text { CP } & \text { Concentrated Phosphate Waste } \\ \text { DC } & \text { Dilute Complexed Waste } \\ \text { DN } & \text { Dilute Non-Complexed Waste } \\ \text { DSS } & \text { Double-Shell Slurry } \\ \text { DSSF } & \text { Double-Shell Slurry Feed } \\ \text { NCPLX } & \text { Non-Complexed Waste } \\ \text { PD/PN } & \text { Plutonium-Uranium Extraction (PUREX) Neutralized Cladding } \\ & \text { Removal Waste (NCRW) transuranic waste (TRU) } \\ \text { PT } & \text { Plutonium Finishing Plant (PFP) TRU Solids }\end{array}$

TANK USE (DOUBLE-SHELL TANKS ONLY)

$\begin{array}{ll}\text { CWHT } & \text { Concentrated Waste Holding Tank } \\ \text { DRCVR } & \text { Dilute Receiver Tank } \\ \text { EVFD } & \text { Evaporate Feed Tank } \\ \text { SRCVR } & \text { Slurry Receiver Tank }\end{array}$

2. SOLID AND LIQUID VOLUME DETERMINATION METHODS
F Food Instrument Company (FIC) Automatic Surface Level Gauge
$M \quad$ Manual Tape Surface Level Gauge
P Photo Evaluation
S Sludge Level Measurement Device

\section{DEFINITIONS}

\section{WASTE TANKS - GENERAL}

Waste Tank Safety Issue

A potentially unsafe condition in the handling of waste material in underground storage tanks that requires corrective action to reduce or eliminate the unsafe condition.

\section{Watch List Tank}

An underground storage tank containing waste that requires special safety precautions because it may have a serious potential for release of high level radioactive waste because of uncontrolled increases in temperature or pressure. Special restrictions have been placed on these tanks by "Safety Measures for Waste Tanks at Hanford Nuclear Reservation," Section 3137 of the National Defense Authorization Act for Fiscal Year 1991, November 5, 1990, Public Law 101-510, (also known as the Wyden Amendment). 


\section{WASTE TYPES}

Aging Waste (AGING)

High level, first cycle solvent extraction waste from the PUREX plant (NCAW)

Concentrated Complexant (CC)

Concentrated product from the evaporation of dilute complexed waste.

Concentrated Phosphate Waste (CP)

Waste originating from the decontamination of the $N$ Reactor in the $100 \mathrm{~N}$ Area. Concentration of this waste produces concentrated phosphate waste.

\section{Dilute Complexed Waste (DC)}

Characterized by a high content of organic carbon including organic complexants:

ethylenediaminetetra-acetic acid (EDTA), citric acid, and hydroxyethyl-ethylenediaminetriacetic acid (HEDTA), being the major complexants used. Main sources of DC waste in the DST system are saltwell liquid inventory (from SSTs).

Dilute Non-Complexed Waste (DN)

Low activity liquid waste originating from $T$ and $S P l$ ants, the 300 and 400 Areas, PUREX facility (decladding supernatant and miscellaneous wastes), $100 \mathrm{~N}$ Area (sulfate waste), B Plant, saltwells, and PFP (supernate).

Double-Shell slurry (DSS)

Waste that exceeds the sodium aluminate saturation boundary in the evaporator without exceeding receiver tank composition limits. For reporting purposes, DSS is considered a solid.

Double-Shell slurry (DSSF)

Waste concentrated just before reaching the sodium aluminate saturation boundary (of 6.5 molar hydroxide) in the evaporator without exceeding receiver tank composition limits. This form is not as concentrated as DSS.

Non-complexed (NCPLX) General waste term applied to all Hanford Site (NCPLX) liquors not identified as complexed.

PUREX Decladding (PD/PN)

PUREX Neutralized Cladding Removal Waste (NCRW) is the solids portion of the PUREX $p$ lant neutralized cladding removal waste stream; received in Tank Farms as a slurry. NCRW solids are classified as transuranic (TRU) waste.

PFP TRU Solids (PT)

TRU solids fraction from PFP Plant operations. 
Drainable Interstitial Liquid (DIL)

Interstitial 1iquid that is not held in place by capillary forces, and will therefore migrate or move by gravity. (See also

Section 4)

\section{Supernate}

The liquid above the solids in waste storage tanks. (See also Section 4)

Ferrocyanide

A compound of iron and cyanide commoniy expressed as $\mathrm{FeCN}$. The actual formula for the ferrocyanide anion is $\left[\mathrm{Fe}(\mathrm{CN})_{6}\right]^{-4}$.

\section{HASTE STATUS}

In-Service Tank

The waste classification of a tank being used, or planned for use, for the storage of liquid (in excess of a minimum supernatant liquid heel) in conjunction with production and/or waste processing.

Out-of-Service Tank

A tank which does not meet the definition of an in-service tank. Before September 1988, these tanks were defined as inactive in this report. (Note: All single-shell tanks are out of service.)

\section{INTERIM STABILIZATION (Single-Shell Tanks only)}

Interim Stabilized (IS)

A tank which contains less than 50,000 gal of drainable

interstitial liquid and less than 5,000 gal of supernatant 1 iquid. If the tank was jet pumped to achieve interim stabilization, then the jet pump flow must also have been at or below $0.05 \mathrm{gpm}$ before interim stabilization criteria is met.

Jet Pump

The jet pump system includes 1) a jet assembly with foot valve mounted to the base of two pipes that extend from the top of the well to near the bottom of the well casing inside the saltwell screen, 2) a centrifugal pump to supply power fluid to the downhole jet assembly, 3) flexible or rigid transfer jumpers, 4) a flush line, and 5) a flowmeter. The jumpers contain piping, valves, and pressure and limit switches.

The centrifugal pump and jet assembly are needed to pump the interstitial liquid from the saltwell screen into the pump pit, nominally a 40-foot elevation rise. The power fluid passes through a nozzle in the jet assembly and acts to convert fluid pressure head to velocity head, thereby reducing the pressure in the jet assembly chamber. The reduction in pressure allows the interstitial liquid to enter the jet assembly chamber and mix with the power fluid. Velocity head is converted to pressure head above the nozzle, lifting power fluid, and interstitial liquid to the pump pit. Pumping rates vary from $0.05 \mathrm{gal}$ to about $4 \mathrm{gal} / \mathrm{min}$. 


\section{Saltwell Screen}

The saltwell system is a 10-inch diameter saltwell casing consisting of a stainless steel saltwell screen welded to a Schedule 40 carbon steel pipe. The casing and screen are to be inserted into the 12-inch tank riser located in the pump pit. The stainless steel screen portion of the system will extend through the tank waste to near the bottom of the tank. The saltwell screen portion of the casing is an approximately 10-foot length of 300 Series, 10-inch diameter, stainless steel pipe with screen openings (slots) of 0.05 inches.

\section{Emergency Pumping Trailer}

A 45-foot Tractor-Type trailer is equipped to provide storage space and service facilities for emergency pumping equipment: this consists of two dedicated jet pump jumpers and two jet pumps, piping and dip tubes for each, two submersible pumps and attached piping, and a skid-mounted Weight Factor Instrument Enclosure (WFIE) with an air compressor and electronic recording instruments. The skid also contains a power control station for the pumps, pump pit leak detection, and instrumentation. A rack for over 100 feet of overground double-contained piping is also in the trailer.

\section{INTRUSION PREVENTION (ISOLATION) Single-Shell Tanks only}

\section{Partially Interim Isolated (PI)}

The administrative designation reflecting the completion of the physical effort required for Interim Isolation except for isolation of risers and piping that is required for jet pumping or for other methods of stabilization.

\section{Interim Isolated (II)}

The administrative designation reflecting the completion of the physical effort required to minimize the addition of liquids into an inactive storage tank, process vault, sump, catch tank, or diversion box. In June 1993, Interim Isolation was replaced by Intrusion Prevention.

Intrusion Prevention (IP)

Intrusion Prevention is the administrative designation reflecting the completion of the physical effort required to minimize the addition of liquids into an inactive storage tank, process vault, sump, catch tank, or diversion box. Under no circumstances are electrical or instrumentation devices disconnected or disabled during the intrusion prevention process (with the exception of the electrical pump), in accordance with WHC-SD-WM-SAR-006 REV 2 , Single-Shell Tank Isolation Safety Analysis Report, March 1986.

\section{TANK INTEGRITY}

Sound

The integrity classification of a waste storage tank for which surveillance data indicate no loss of liquid attributed to a breach of integrity. 


\section{Assumed Leaker}

The integrity classification of a waste storage tank for which surveillance data indicate a loss of 1 iquid attributed to a breach of integrity.

\section{Assumed Re-Leaker}

A condition that exists after a tank has been declared a:s an "assumed leaker" and then the surveillance data indicates a new loss of liquid attributed to a breach of integrity.

\section{IANK INVESTIGATION}

\section{Intrusion}

A term used to describe the inflitration of liquid into a waste tank.

\section{SURVEILLANCE INSTRUMENTATION}

Drywells

Drywells are vertical boreholes with 6-in. (internal diameter) carbon steel casings positioned radially around SSTs. Periodic monitoring is done by gamma radiation or neutron sensors to obtain scan profiles of radiation or moisture in the soil as a function of well depth, which could be indicative of tank leakage. These wells range between 50 and $250 \mathrm{ft}$ in depth, and are monitored between the range of 50 to $150 \mathrm{ft}$. The wells are sealed when not in use. They are called drywells because they do not penetrate to the water table and are therefore usually "dry." There are 759 drywells which are monitored on various frequencies.

\section{Laterals}

Laterals are horizontal drywells positioned under single-shell waste storage tanks to detect radionuclides in the soil which could be indicative of tank leakage. These drywells are monitored by radiation detection probes. Laterals are 4-in. inside diameter steel pipes located 8 to $10 \mathrm{ft}$ below the tank's concrete base. There are three laterals per tank. Laterals are located only in $\mathrm{A}$ and SX farms.

\section{Surface Levels}

The surface level measurements in all waste storage tanks are monitored by manual or automatic conductivity probes, and recorded and transmitted or entered into the Computer Automated Surveillance System (CASS).

\section{Automatic FIC}

An automatic waste surface level measurement device is manufactured by the Food Instrument Company (FIC). The instrument consists of a conductivity electrode ( $p l$ ummet) connected to a calibrated steel tape, a steel tape reel housing and a controller that automatically raises and lowers the plummet to obtain a waste surface level reading. The controller can provide a digital display of the data 
and also transmit the reading to the CASS. Some tanks have gauges connected to CASS and others are read manually.

Annulus

The annulus is the space between the inner and outer shells on DSTs only. Drain channels in the insulating and/or supporting concrete carry any leakage to the annulus space where conductivity probes are installed. Alarms from the annunciators are received by CASS. Continuous Air Monitoring (CAM) alarms are also located in the annulus. The annulus conductivity probes and radiation detectors are the primary means of leak detection for all DSTs.

\section{Liquid Observation Well (LOW)}

In-tank liquid observation wells are used for monitoring the interstitial liquid level (ILL) in single-shell waste storage tanks. The wells are usually constructed of fiberglass or TEFZEL*reinforced epoxy-polyester resin. There are a few LOWs constructed of steel. LOWs are sized to extend to within 1 in. of the bottom of the waste tank, are sealed at their bottom ends and have a nominal outside diameter of $3.5 \mathrm{in}$. Two probes are used to monitor changes in the ILL; gamma and neutron, which can indicate intrusions or leakage by increases or decreases in the ILL. There are 58 LOWs (56 are in operation) installed in SSTs that contain or are capable of containing greater than $50,000 \mathrm{gal}$ of drainable interstitial liquid, and in two DSTs only. The LOWs installed in two DSTs, (102-SY and 103-AW Tanks), are constructed of steel and are used for special, rather than routine, surveillance purposes only.

\section{Thermocouple (TC)}

A thermocouple is a thermoelectric device used to measure temperature. More than one thermocouple on a device (probe) is called a thermocouple tree. In DSTs there may be one or more thermocouple trees in risers in the primars' tank. In addition, in DSTs only, there are thermocouple elements installed in the insulating concrete, the lower primary tank knuckle, the secondary tank concrete foundation, and in the outer structural concrete. These monitor temperature gradients within the concrete walls, bottom of the tank, and the domes. In SSTs, one or more thermocouples may be installed directly in a tank, al though some SSTs do not have any trees installed. A single thermocouple (probe) may be installed in a riser, or lowered down an existing riser or LOW. There are also four thermocouple laterals beneath Tank 105-A in which temperature readings are taken in 34 thermocouples.

\section{In-tank Photography}

In-tank photographs may be taken to aid in resolving in-tank measurement anomalies and determine tank integrity. Photographs help determine sludge and liquid levels by visual examination.

*TEFZEL, a trademark of E. I. du Pont de Nemours \& Company 
WHC-EP-0182-74

\section{TERMS/ACRONYMS}

CASS

OSD

OSR

SAR

TMACS

TPA

USQ

Wyden Amendment
Computer Analysis Surveillance System

Operating Specifications Document

Operational Safety Requirements (OSRs are sections in SARs - see below)

Safety Analysis Reports

Tank Monitor and Control System

Hanford Federal Facility Consent and Compliance Order, "Washington State Department of Ecology, U. S. Environmental Protection Agency, and U. S. Department of Energy," 1992 (Tri-Party Agreement)

Unreviewed Safety Question

"Safety Measures for Waste Tanks at Hanford Nuclear Reservation," Section 3137 of the National Defense Authorization Act for Fiscal Year 1991, November 5, 1990, Public Law 101-510.

4. INVENTORY AND STATUS BY TANK - VOLUME CALCULATIONS/DEFINITIONS FOR TABLE E-5 (SINGLE-SHELL TANKS)

\begin{tabular}{||l|l||}
\hline COLUMN HEADING & \multicolumn{1}{|c|}{ VOLUME CALCULATIONS/DEFINITIONS } \\
\hline \hline Total Waste & $\begin{array}{l}\text { Solids volume plus Supernatant liquid. Solids } \\
\text { include sludge and saltcake (see definitions below) }\end{array}$ \\
\hline Supernatant Liquid & $\begin{array}{l}\text { Drainable Liquid Remaining minus Drainable } \\
\text { Interstitial Supernate is the clear liquid } \\
\text { floating on the surface of the waste. Supernate is } \\
\text { usually derived by subtracting the solids level } \\
\text { measurement from the liquid level measurement. In } \\
\text { some cases, the supernatant volume includes } \\
\text { floating solid crusts because their volume cannot } \\
\text { be measured. Photographs are useful in estimating } \\
\text { the liquid volumes; the area of solids covered and } \\
\text { the average depth can be estimated. }\end{array}$ \\
\hline
\end{tabular}




\begin{tabular}{|c|c|}
\hline COLUMN HEADING & VOLUME CALCULATIONS/DEFINITIONS \\
\hline $\begin{array}{l}\text { Drainable } \\
\text { Interstitial } \\
\text { Liquid }\end{array}$ & $\begin{array}{l}\text { Drainable Liquid Remaining minus Supernate. } \\
\text { Drainable interstitial liquid is calculated based } \\
\text { on the saltcake and sludge volumes, using average } \\
\text { porosity values or actual data for each tank, when } \\
\text { available. Interstitial liquid is liquid that } \\
\text { fills the interstitial spaces of the solids waste. } \\
\text { Drainable interstitial liquid is calculated based } \\
\text { on the saltcake and sludge volumes in the tank. } \\
\text { The sum of the interstitial liquid contained in } \\
\text { saltcake and sludge is the initial volume of } \\
\text { drainable interstitial liquid. The volume reported } \\
\text { as Drainable Interstitial Liquid is the initial } \\
\text { volume of drainable interstitial liquid minus } \\
\text { interstitial liquid removed by pumping. }\end{array}$ \\
\hline Pumped This Month & $\begin{array}{l}\text { Net total gallons of liquid pumped from the tank } \\
\text { during the month. If supernate is present, pump } \\
\text { production is first subtracted from the supernatant } \\
\text { volume. The remainder is then subtracted from the } \\
\text { drainable interstitial liquid volume. The total } \\
\text { pumped volume is subtracted from drainable liquid } \\
\text { remaining and pumpable liquid remaining. Pump } \\
\text { production takes into account the amount of water } \\
\text { added to the tank during the month (if any). }\end{array}$ \\
\hline Total Pumped & $\begin{array}{l}\text { Cumulative net total gallons of liquid pump from } \\
1979 \text { to date. }\end{array}$ \\
\hline $\begin{array}{l}\text { Drainable Liquid } \\
\text { Remaining }\end{array}$ & $\begin{array}{l}\text { Supernate plus Drainable interstitial. (See } \\
\text { Supernatant Liquid and Drainable Interstitial } \\
\text { Liquid above for definitions). The total Drainable } \\
\text { Liquid Remaining is the sum of drainable } \\
\text { interstitial liquid and supernate minus total } \\
\text { gallons pumped. }\end{array}$ \\
\hline $\begin{array}{l}\text { Pumpable Liquid } \\
\text { Remaining }\end{array}$ & $\begin{array}{l}\text { Drainable Liquid Remaining minus undrainable heel } \\
\text { volume. (Dish bottom tanks have a "heel" where } \\
\text { liquids can collect: flat bottom tanks do not). } \\
\text { (See Drainable Liquid Remaining and Pumped this } \\
\text { Month for definitions). Not all drainabie } \\
\text { interstitial liquid is pumpable. It is assumed } \\
\text { that drainable interstitial liquid on top of the } \\
\text { undrainable heel in sludge or saltcake, is not jet } \\
\text { pumpable. Therefore, pumpable interstitial liquid } \\
\text { is the initial volume of drainable interstitial } \\
\text { liquid minus the amount of interstitial liquid on } \\
\text { top of the heel. The volume shown as Pumpable } \\
\text { Liquid Remaining is the sum of pumpable } \\
\text { interstitial liquid and supernate minus total } \\
\text { gallons pumped. }\end{array}$ \\
\hline
\end{tabular}




\begin{tabular}{|c|c|}
\hline COLUMN HEADING & VOLUME CALCULATIONS/DEFINITIONS \\
\hline Sludge & $\begin{array}{l}\text { Solids formed during sodium hydroxide additions to } \\
\text { waste. Sludge usually was in the form of suspended } \\
\text { solids when the waste was originally received in } \\
\text { the tank from the waste generator. In-tank } \\
\text { photographs may be used to estimate the volume. }\end{array}$ \\
\hline Saltcake & $\begin{array}{l}\text { Results from crystallization and precipitation } \\
\text { after concentration of liquid waste, usually in an } \\
\text { evaporator. If saltcake is layered over sludge, it } \\
\text { is only possible to measure total solids volume. } \\
\text { in-tank photographs may be used to estimate the } \\
\text { saltcake volume. }\end{array}$ \\
\hline Solids Volume Update & $\begin{array}{l}\text { Indicates the latest update of any change in the } \\
\text { solids volume. }\end{array}$ \\
\hline $\begin{array}{l}\text { Solids Update Source } \\
\text { - See Footnote }\end{array}$ & $\begin{array}{l}\text { Indicates the source or basis of the latest solids } \\
\text { volume update. }\end{array}$ \\
\hline Last Photo Date & Date of latest in-tank photographs taken. \\
\hline $\begin{array}{l}\text { See Footnotes for } \\
\text { These Changes }\end{array}$ & $\begin{array}{l}\text { Indicates any change made the previous month. A } \\
\text { footnote explanation for the change follows the } \\
\text { Inventory and Status by Tank section (Table } E-5 \text { ). }\end{array}$ \\
\hline
\end{tabular}


WHC-EP-0182-74

This page intentionally left blank. 
WHC-EP-0182-74

\section{APPENDIX D}

TANK FARM CONFIGURATION, STATUS, AND

FACILITY CHARTS 
WHC-EP-0182-74

This page intentionally left blank.

D-2 


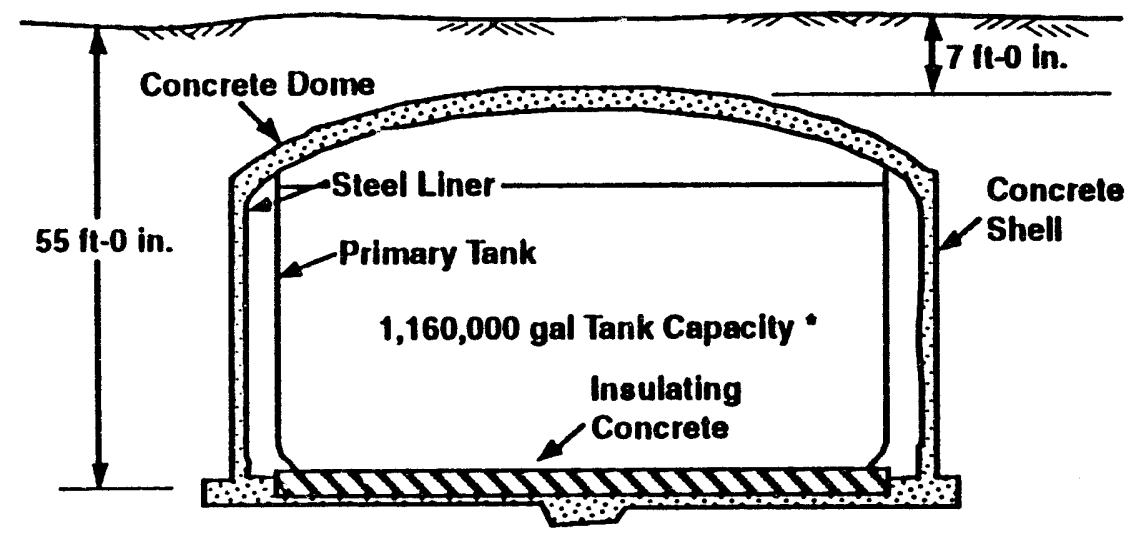

75 it Diameler Double-Shell Tank Tank Farms: AN, AP, AW, AY, AZ, SY

0
$\mathbf{\omega}$

- AY and AZ Have a Tank Capacity of $1,000,000 \mathrm{gal}$

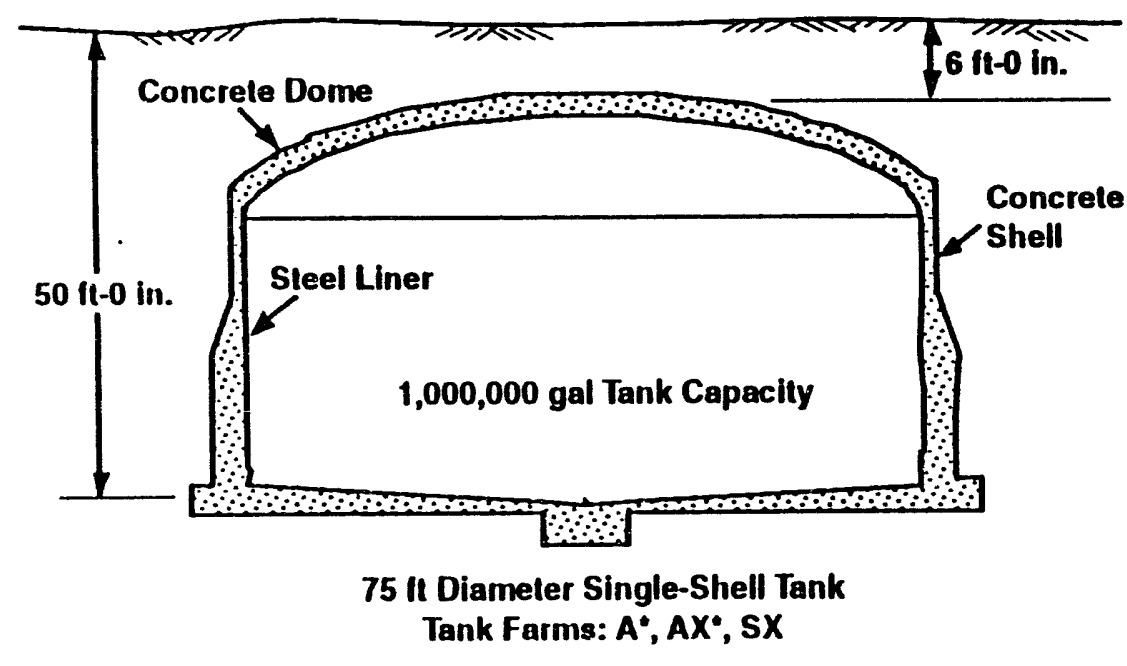

- A and AX have flat bottoms
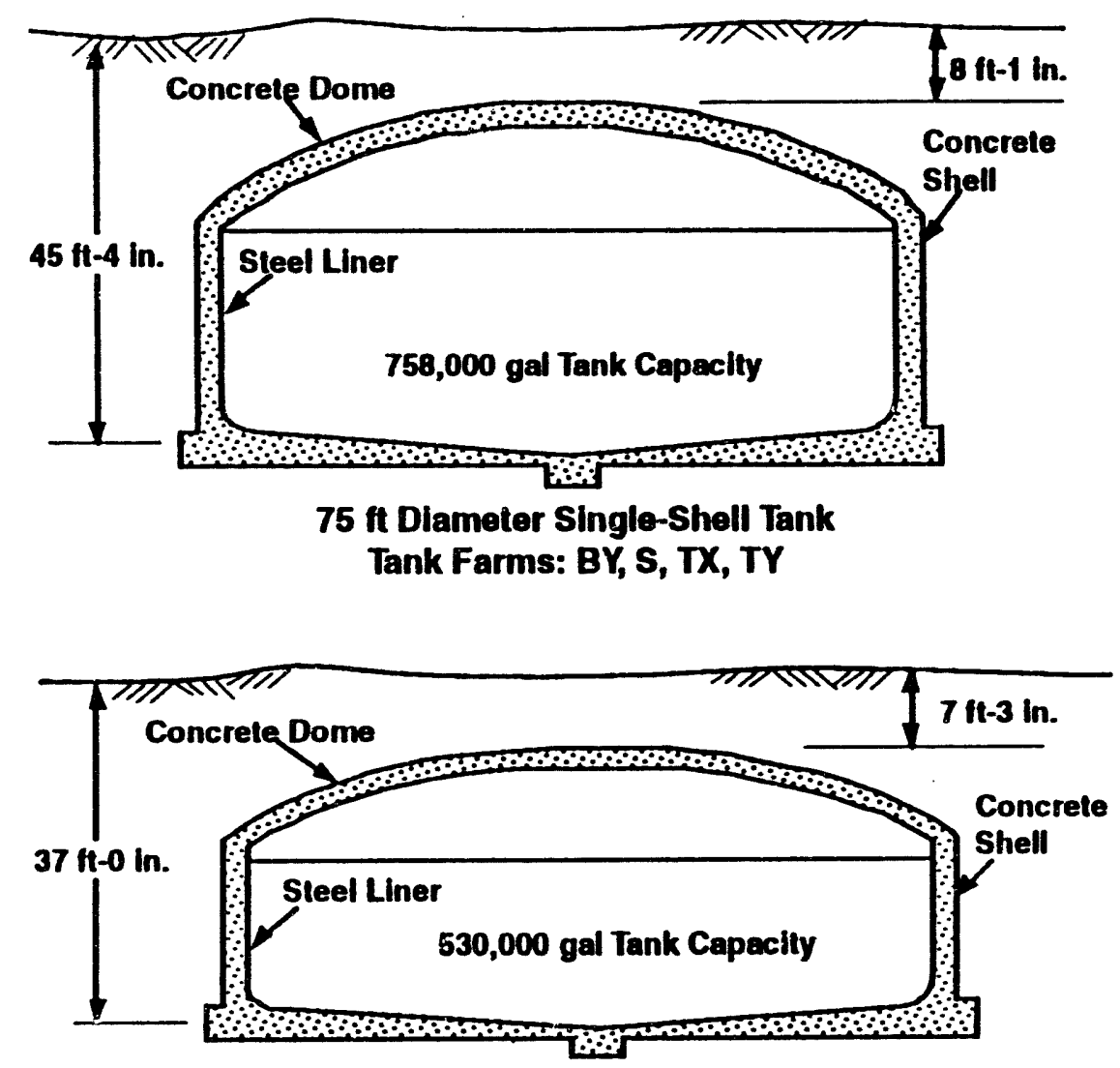

75 ft Diameter Single-Shell Tank Tank Farms: B, BX, C, T, U

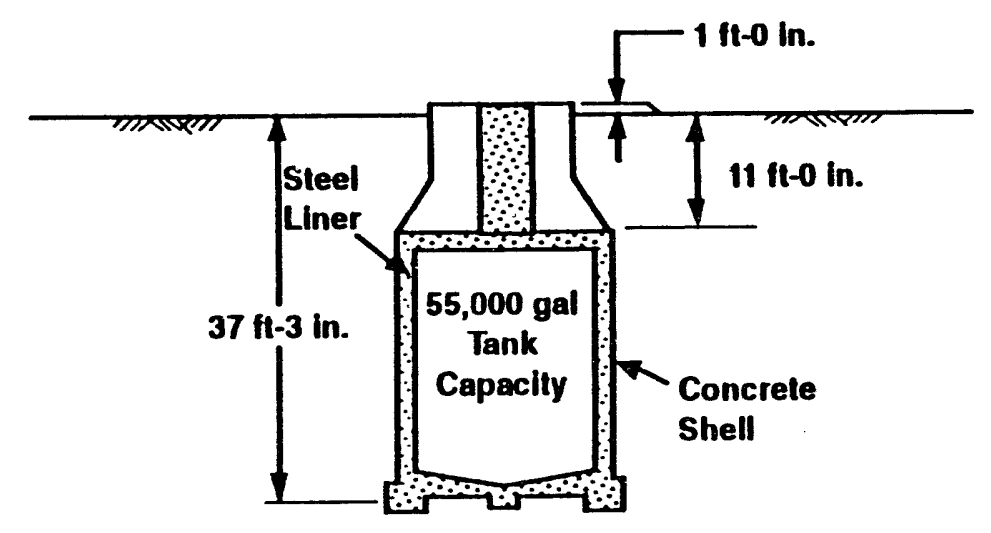

20 ft Diameter Single-Shell Tank Tank Farms: B, C, T, U 


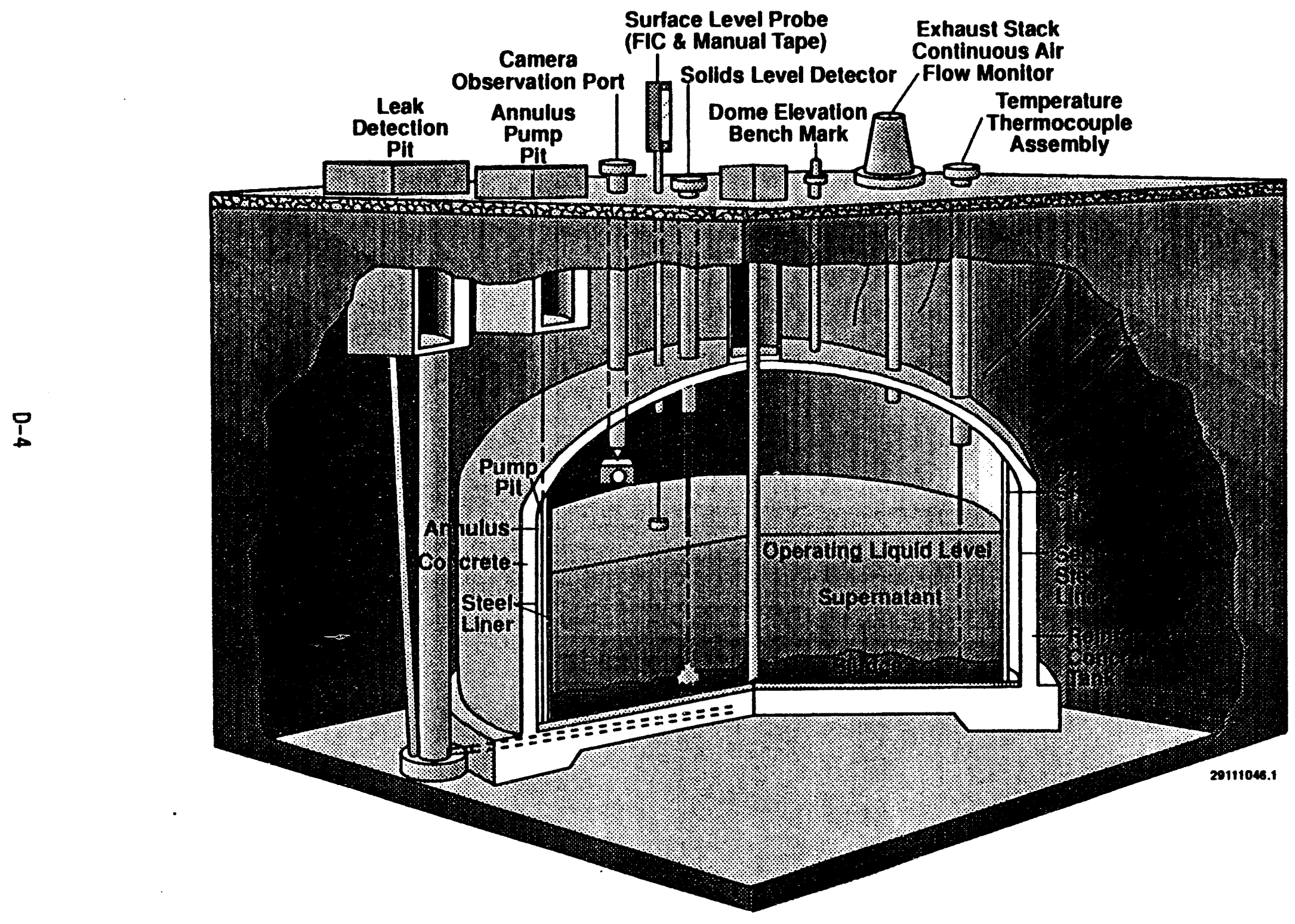

Figure D-2. Double-Shell Tank Instrumentation Configuration 


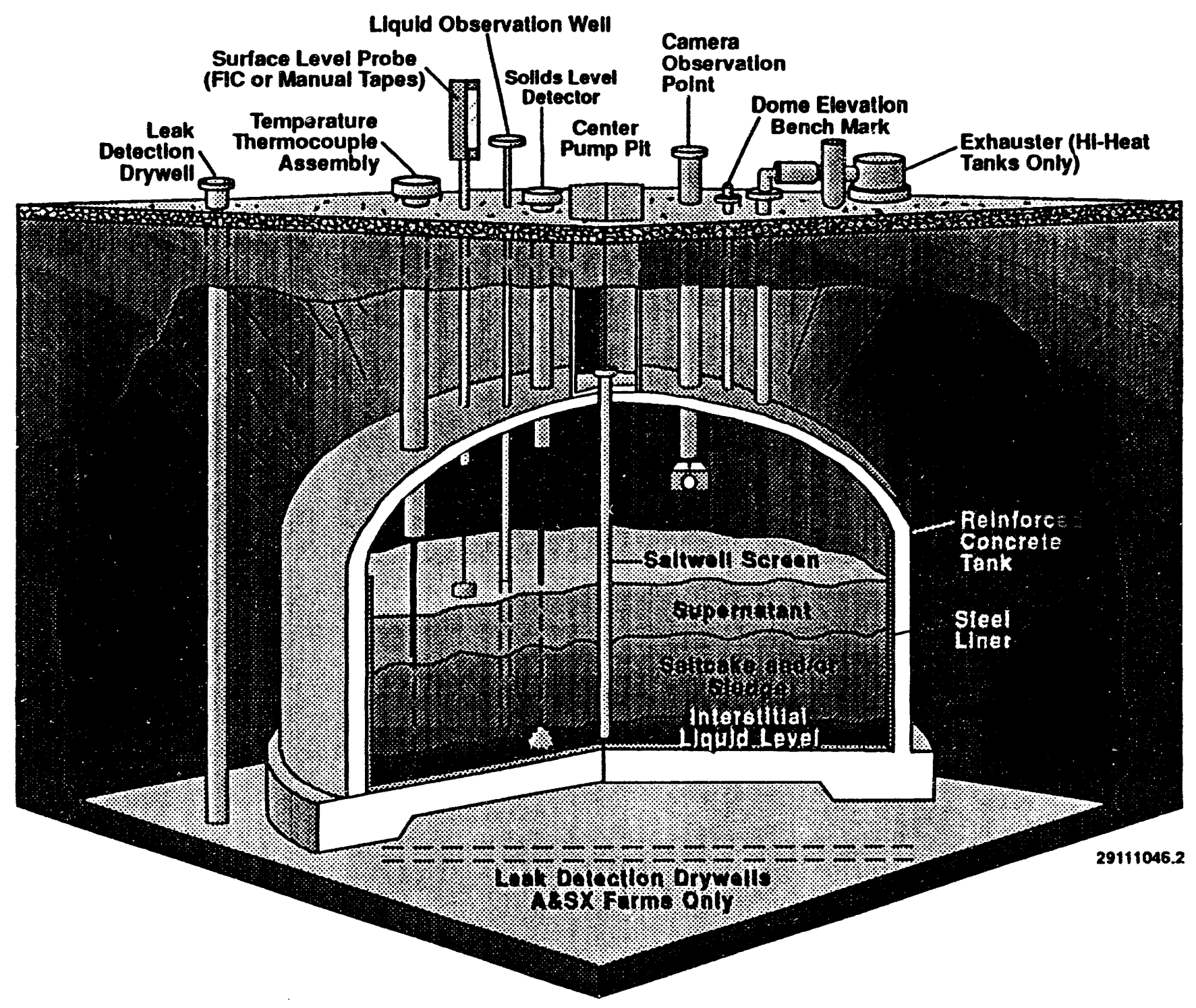

Figure D-3. Single-Shell Tank Instrumentation Configuration 
WHC-EP-0182-74

This page intentionally left blank 


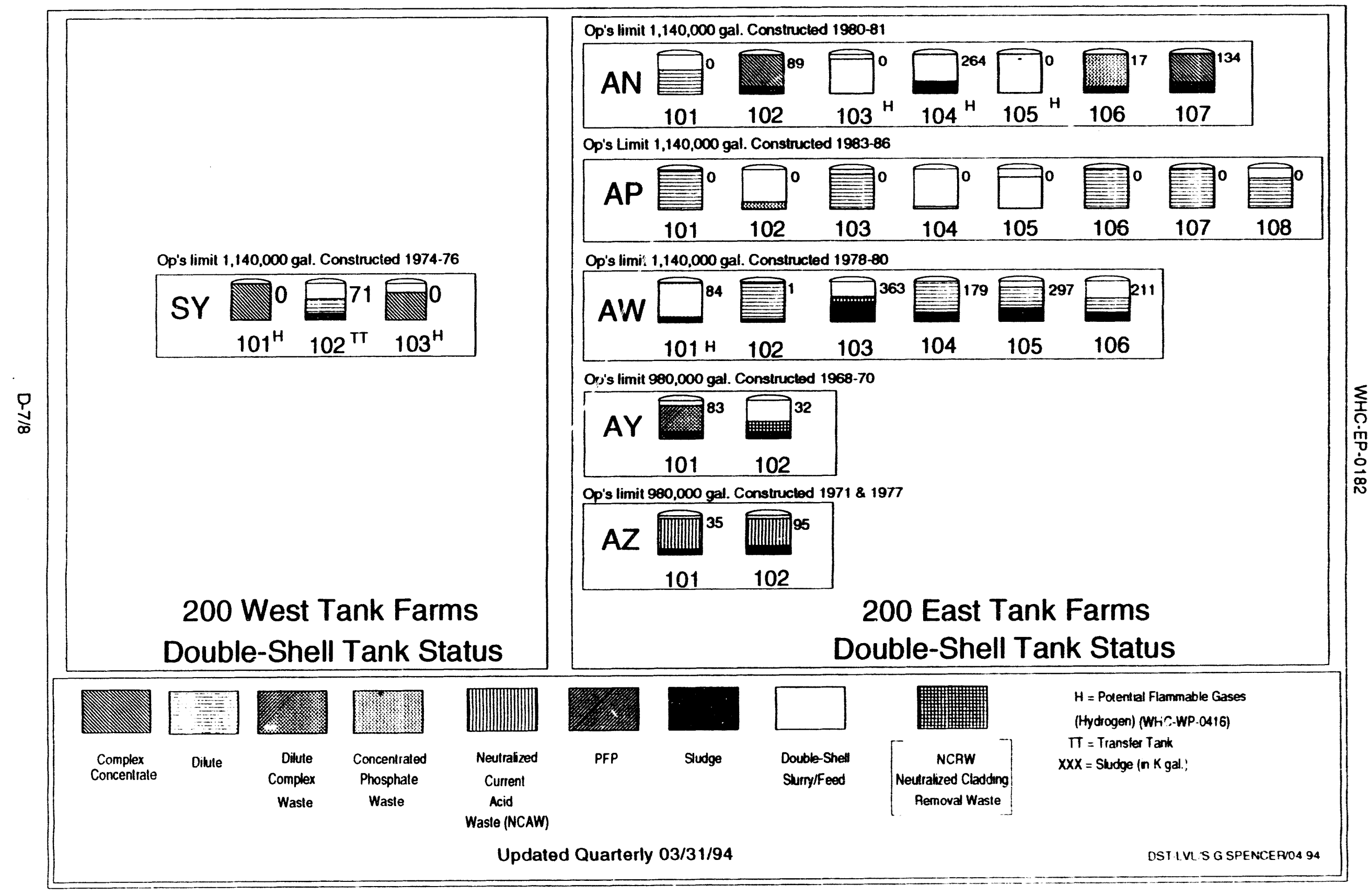

Figure D-4. Double-Shell Tank Status 
$1,000.000$ gal. tanke Conotructed 1964-66

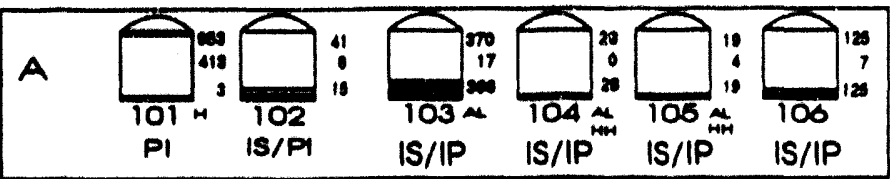

1,000,000 0al. tanks Conatructed 1963-64

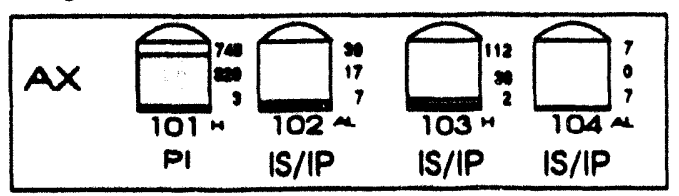

600,000 gal. tanks Continucted 1943-44

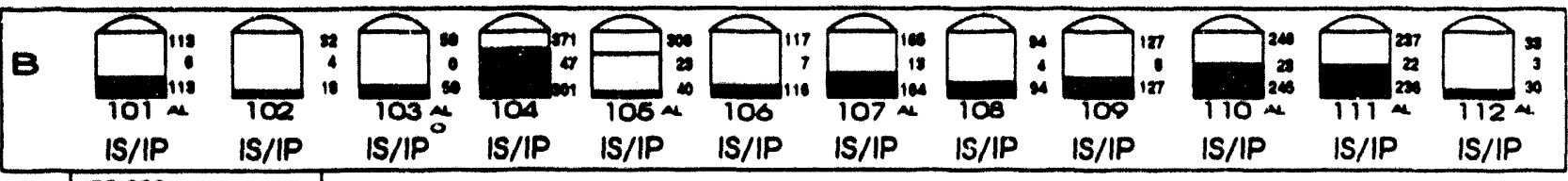

\begin{tabular}{|c|c|c|c|}
\hline \multicolumn{2}{|c|}{86.000 gal. tanks } & & \\
\hline $\begin{array}{l}201 \\
\text { IS/IP }\end{array}$ & $\begin{array}{l} \\
202 \\
\text { IS/IP }\end{array}$ & $\begin{array}{l}203 \\
\text { IS/IP }\end{array}$ & $\begin{array}{l}{ }_{204} \\
\text { IS/IP }\end{array}$ \\
\hline
\end{tabular}

800.000 gal. tanks Contructed 1946-47

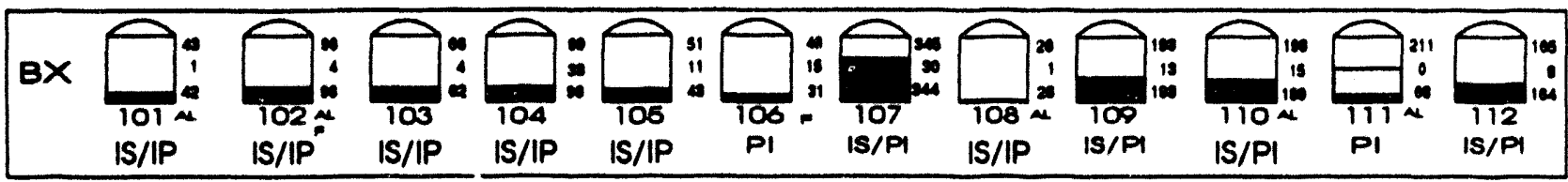

750.000 gal. tanks Conatructed 1948-49

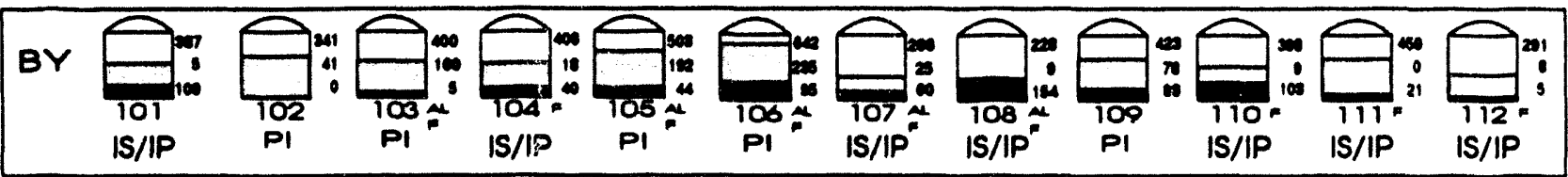

500.000 gal. tanka Conotructed 1943-44

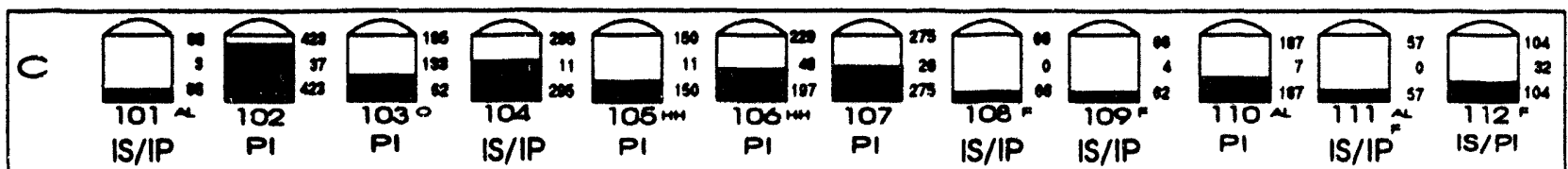

\begin{tabular}{|c|c|c|c|}
\hline \multicolumn{2}{|c|}{56,000 col. tonlat } & & \\
\hline & & 0 & \\
\hline $\begin{array}{l}201 \\
\text { IS/IP }\end{array}$ & $\begin{array}{l}202 \\
\text { IS/IP }\end{array}$ & $\begin{array}{l}200 \\
\text { IS/IP }\end{array}$ & $\begin{array}{l}204 \\
\text { IS/IP }\end{array}$ \\
\hline
\end{tabular}

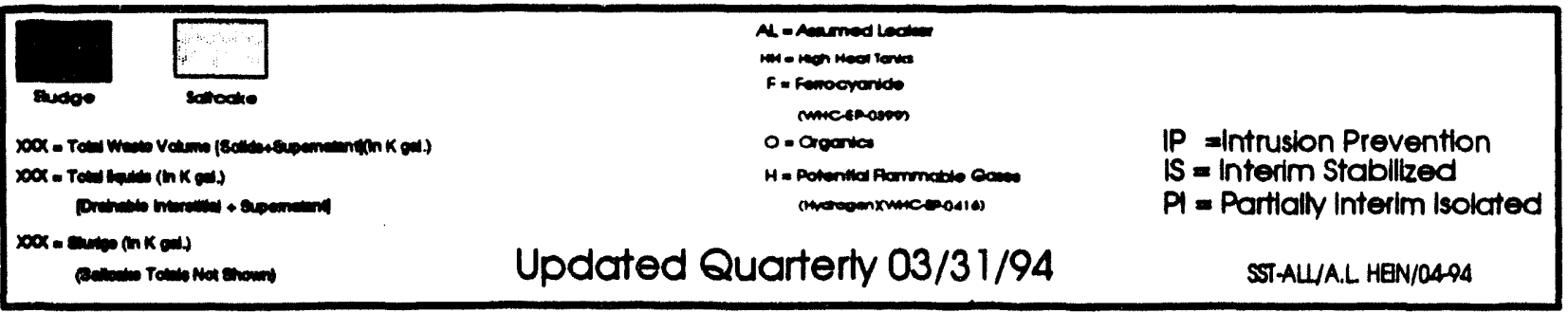

Figure D-5. 200E Single-Shell Tank Status 
760,000 gal, tanks Conatructed $1950-61$

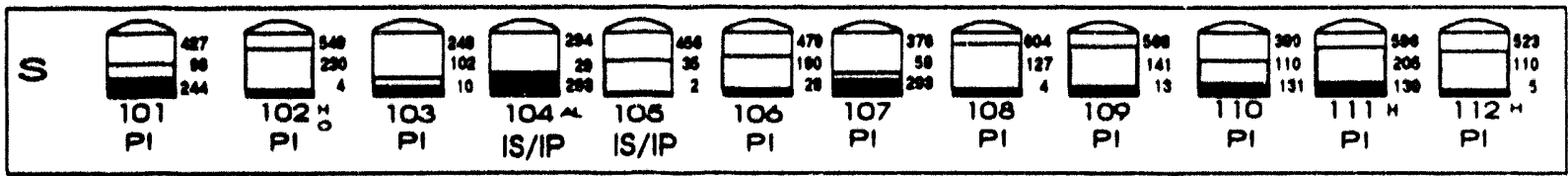

1,000,000 oal. tanks Contructed 1983-64

SX

500,000 gal. tanke Conatucted 1943-44

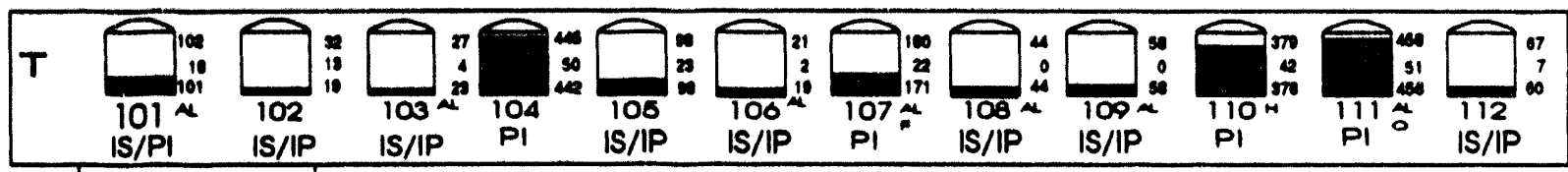

\begin{tabular}{|c|c|c|c|}
\hline \multicolumn{2}{|c|}{ 56,000 gal. tanks } & & \\
\hline $\begin{array}{l}201 \\
1 S / I P\end{array}$ & $\begin{array}{l}202 \\
1 S / 1 P\end{array}$ & $\begin{array}{l}203 \\
1 S / 1 P\end{array}$ & $\begin{array}{l}204 \\
1 S / 1 P\end{array}$ \\
\hline
\end{tabular}

750,000 gal. tonk Contructed 1947-48

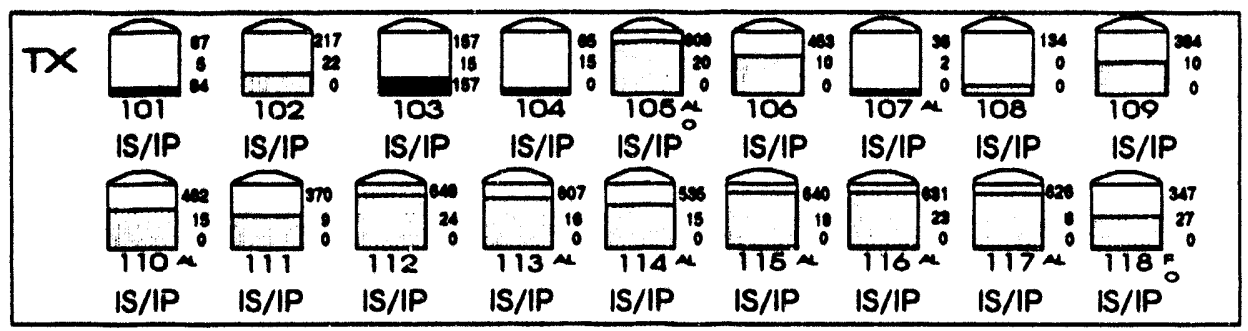

780.000 gal. tanks Constructed 1951-52

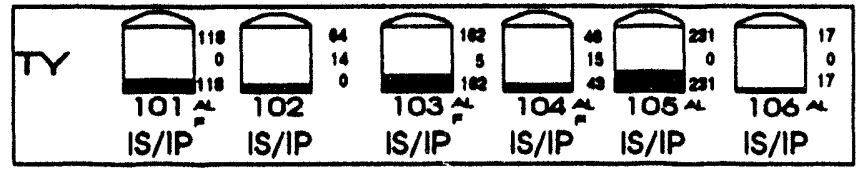

500,000 gal. tanks Constructed $1943-44$

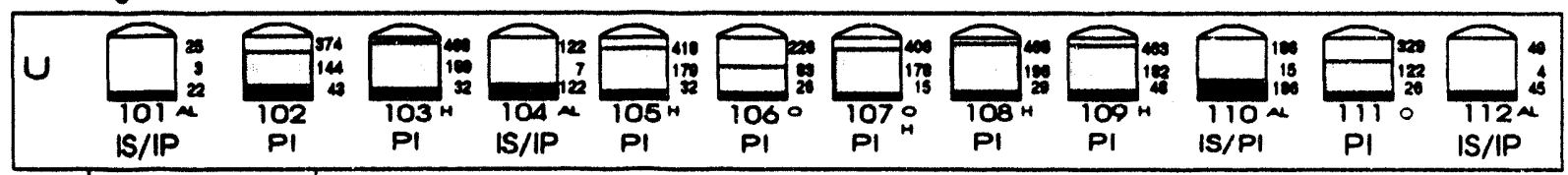

\begin{tabular}{|c|c|c|c|}
\hline \multicolumn{2}{|c|}{56,000 gal. tanks } & & \\
\hline & & & 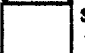 \\
\hline IS/IP & $|S /| P$ & IS/IP & IS/IP \\
\hline
\end{tabular}

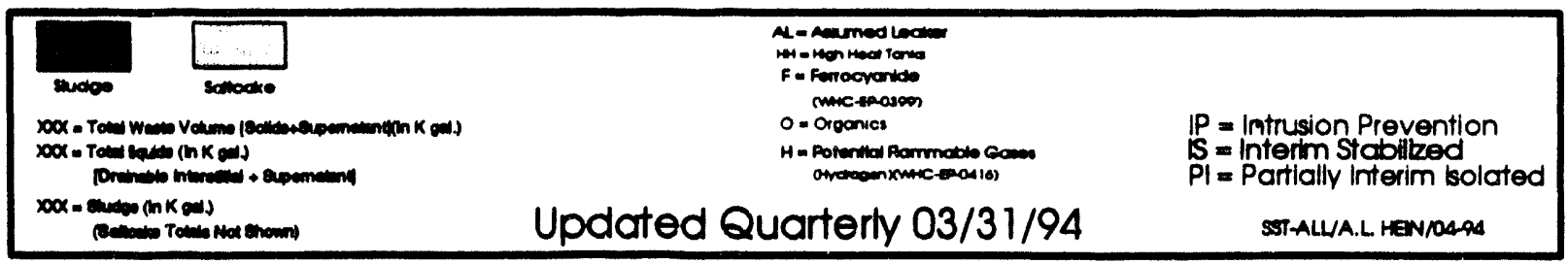

Fig. D-6. 200W Single-Shell Tank Status 


\section{Hanford Tank Farm Facilt 200 East}

Note: All single-shell tanks were removed from service (not allowed to receive waste) on or before November 21, 1980

Single Walled Pipe Direct Buried

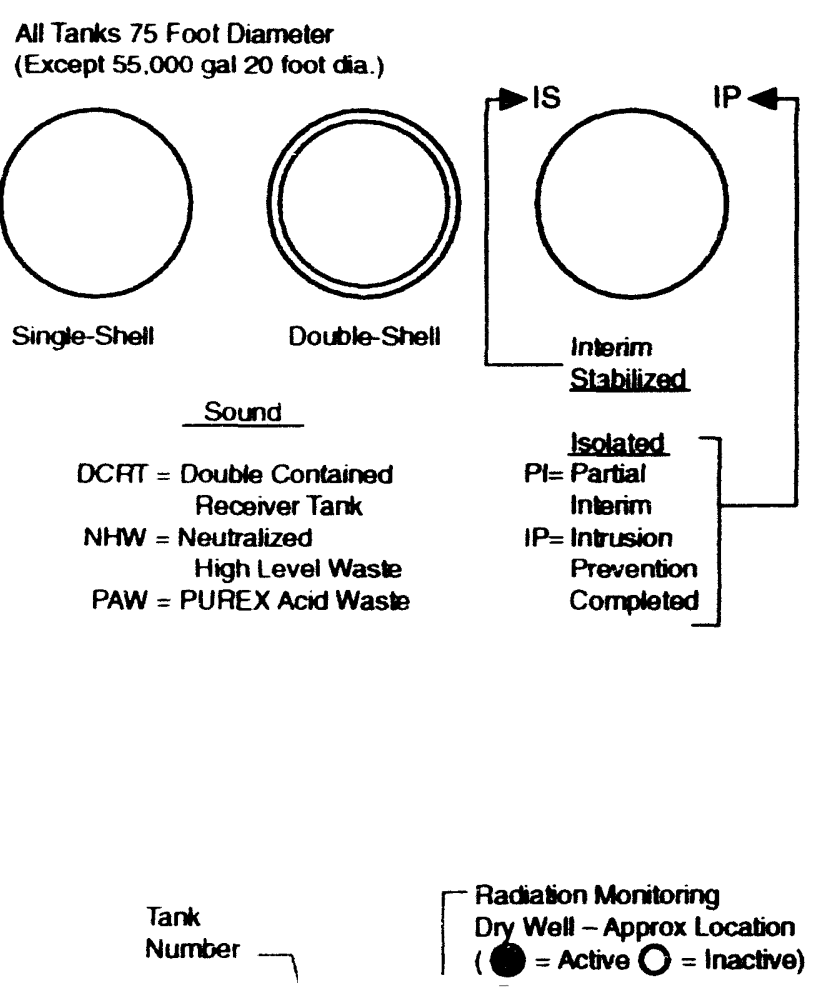

\section{BY-Tank Farm}

1948-49

$12 \odot 758,000 \mathrm{gal}$
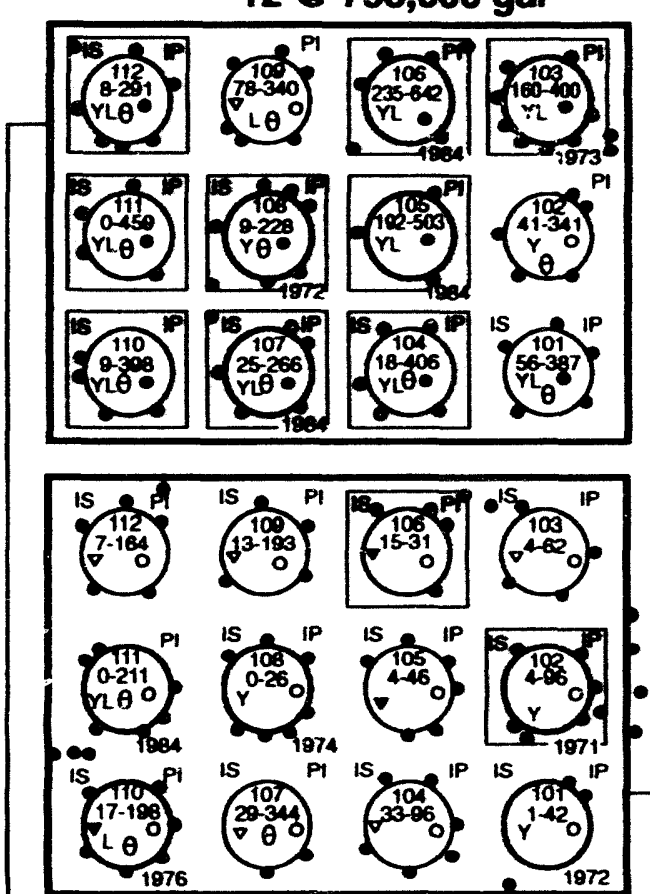

BX-Tank Farm 1946-47

$12 \bigcirc 530,000$ gal
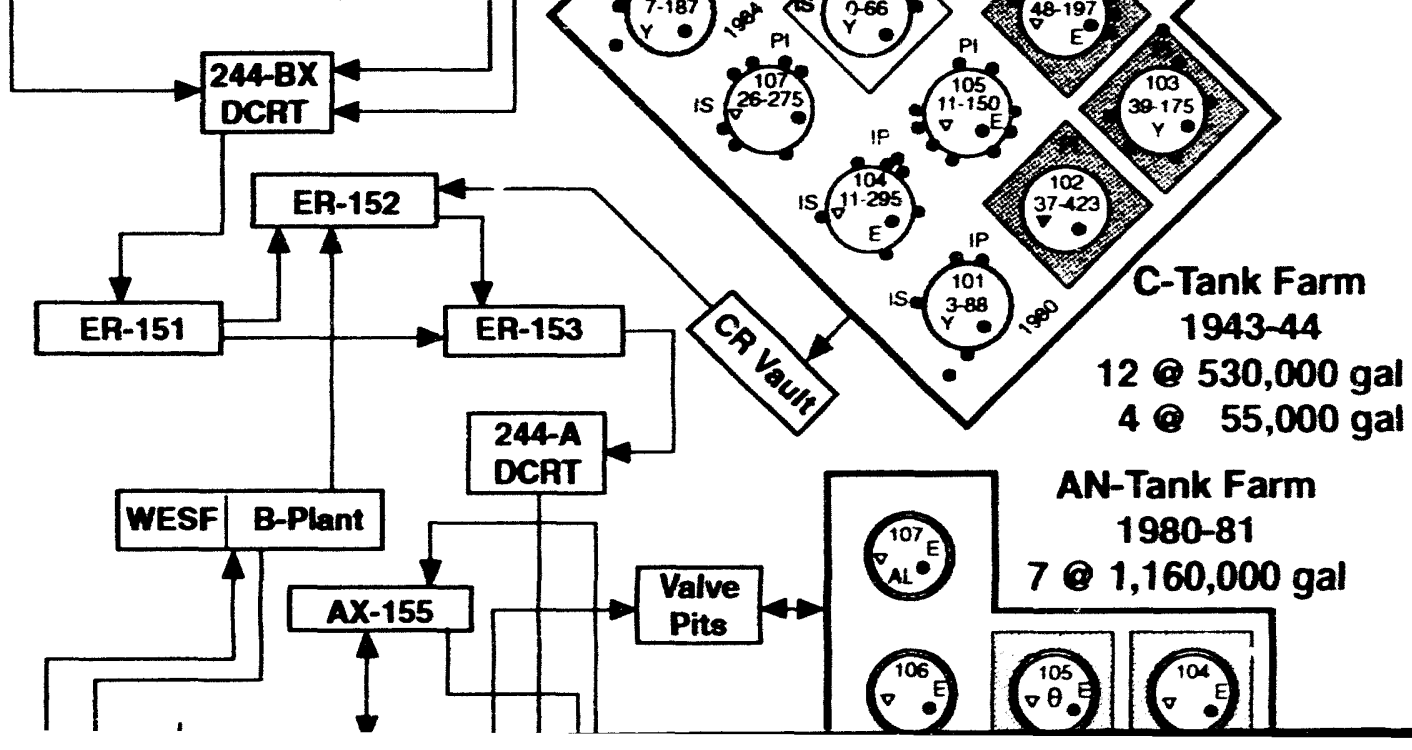

B-Tank Farm

$1943-44$

$12 \odot 530,000 \mathrm{gal}$

$455,000 \mathrm{gal}$

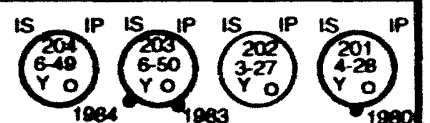

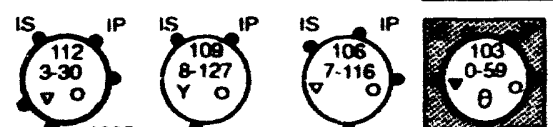

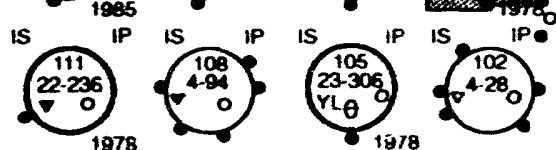

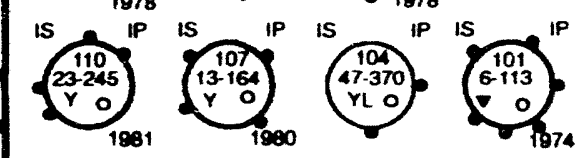
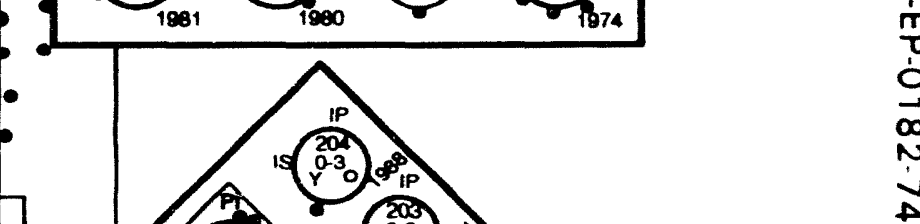

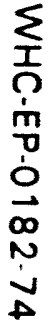

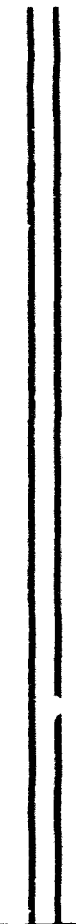




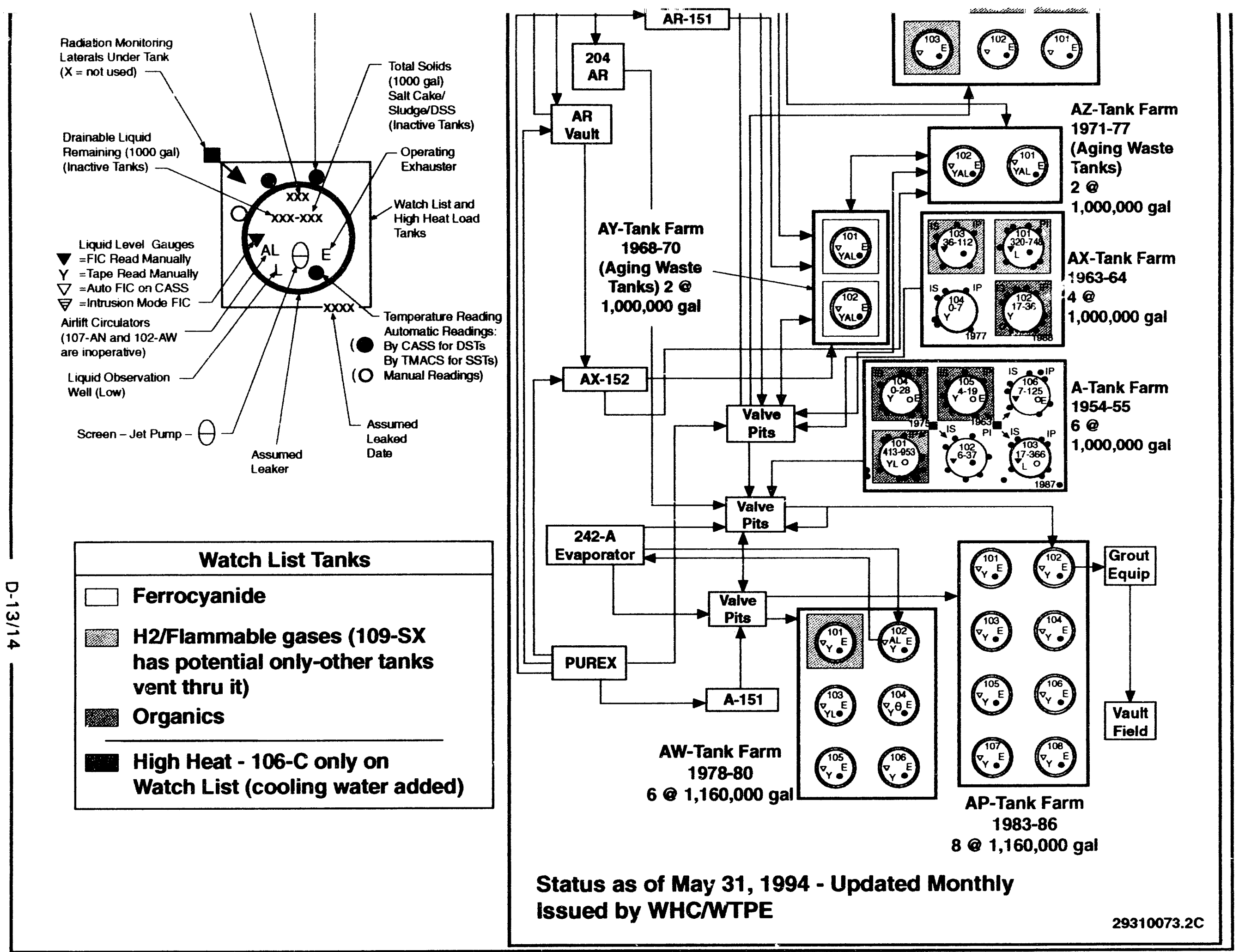




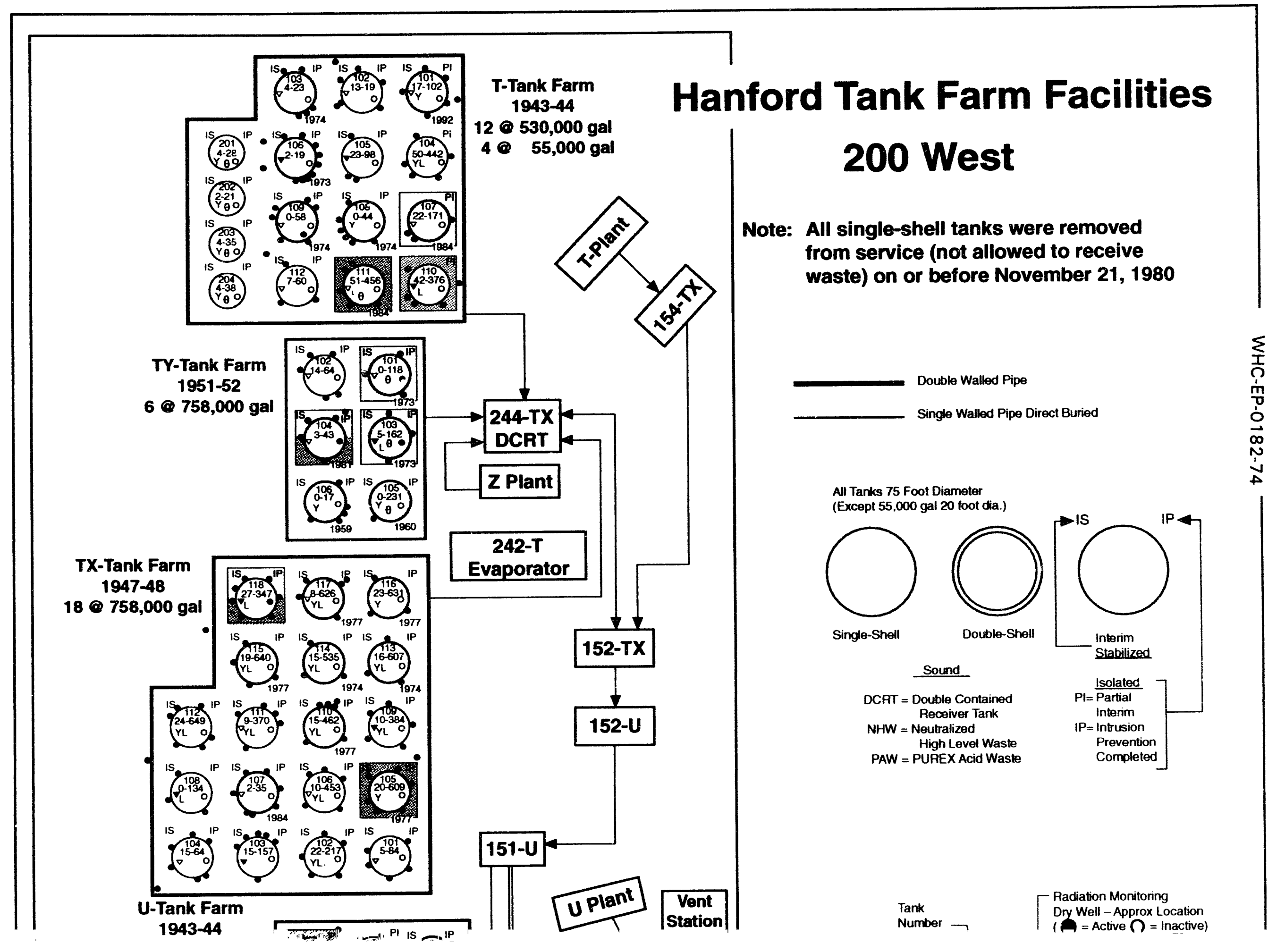


WHC-EP-0182-74

\title{
APPENDIX E
}

\author{
MONTHLY SUMMARY \\ TANK USE SUMMARY \\ INVENTORY SUMMARY BY TANK FARM \\ INVENTORY AND STATUS BY TANK
}


WHC-EP-0182-74

This page intentionally left blank. 


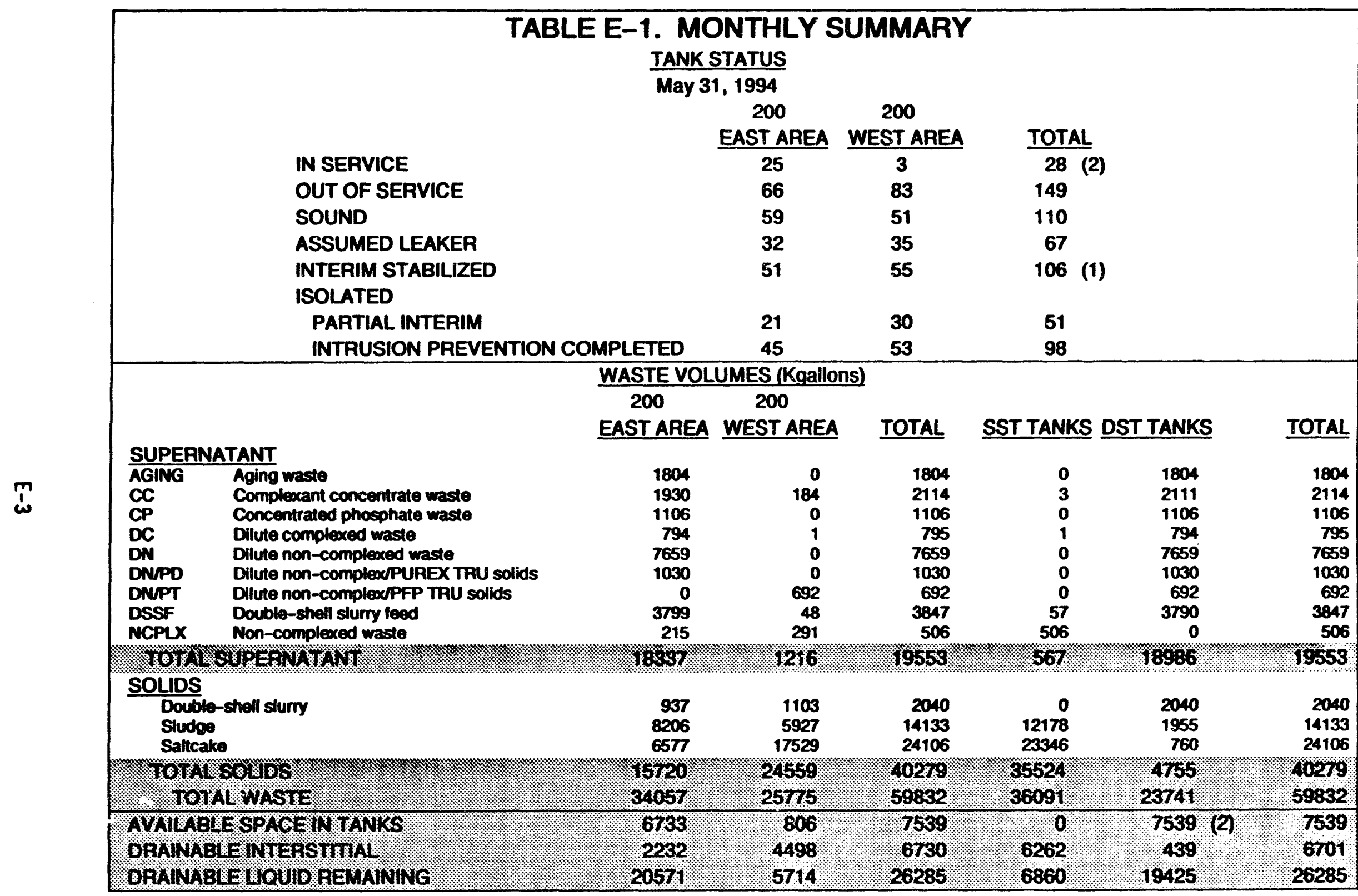

(1) Includes six tanks that do not meet current established supernatant and interstitial liquid stabilization criteria, B-104, 110, 111, T-102, T-112, and U-110. (2) Includes six double-shell tanks on Hydrogen Watch List not currently allowed to receive waste, 103-AN, 104-AN, 105-AN, 101-AW, 101-SY, and 103-SY. Note: $+1-1 \mathrm{Kgal}$ differences are the result of computer rounding 
TABLE E-2. TANK USE SUMMARY

May 31, 1994

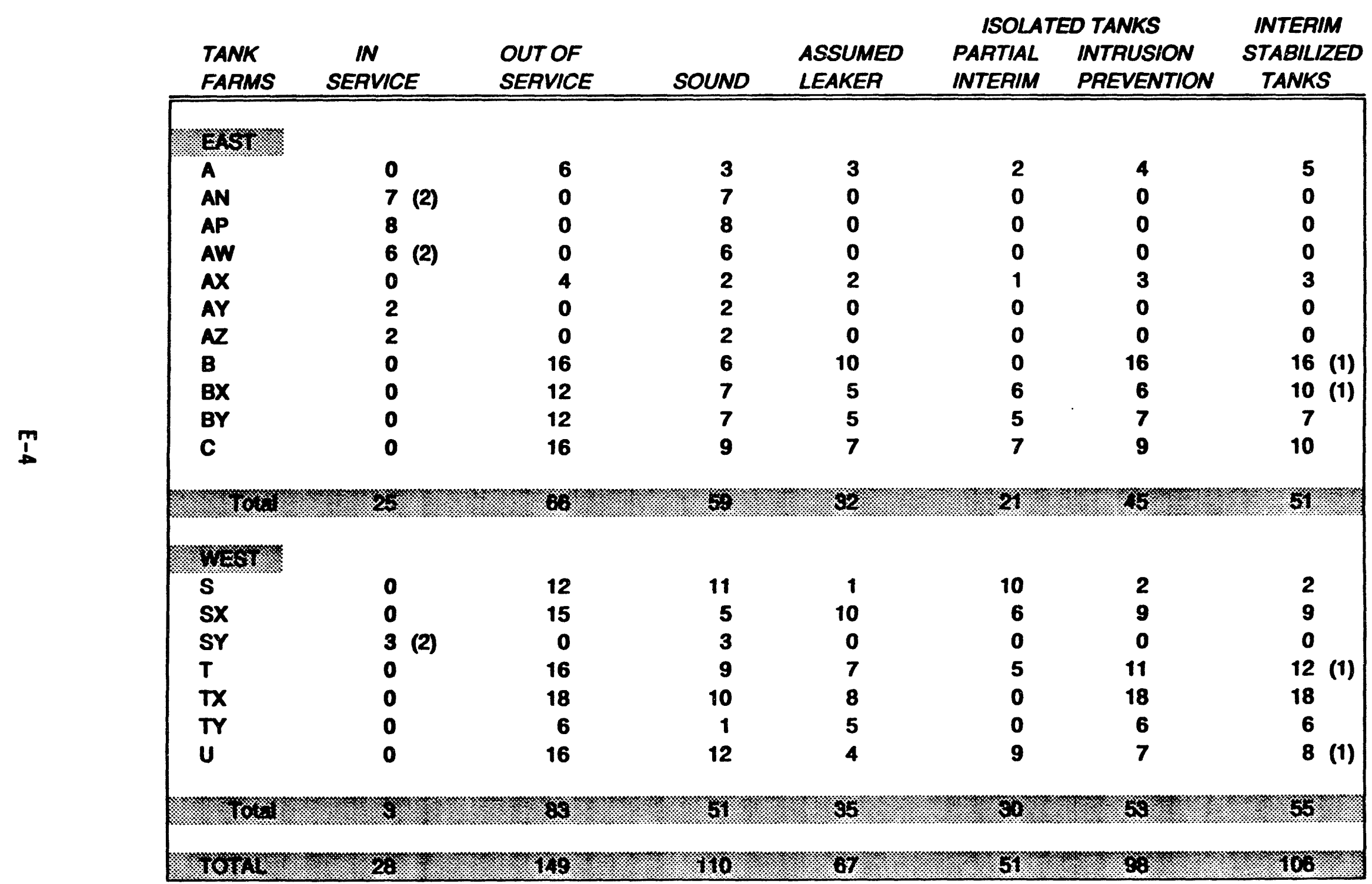

(1) Includes six tanks that do not meet current established supernatant and interstitial liquid stabilization criteria (B-104, 110, 111, T-102, 112. and $\mathrm{U}-110$ ).

(2) Sbx Double-Shell Tanks on the Hydrogen Tank Watch List are not currently in service (AN-103, 104, 105, AW-101, SY-101 and 103). 
TABLE E-3. INVENTORY SUMMARY BY TANK FARM

May 31, 1994

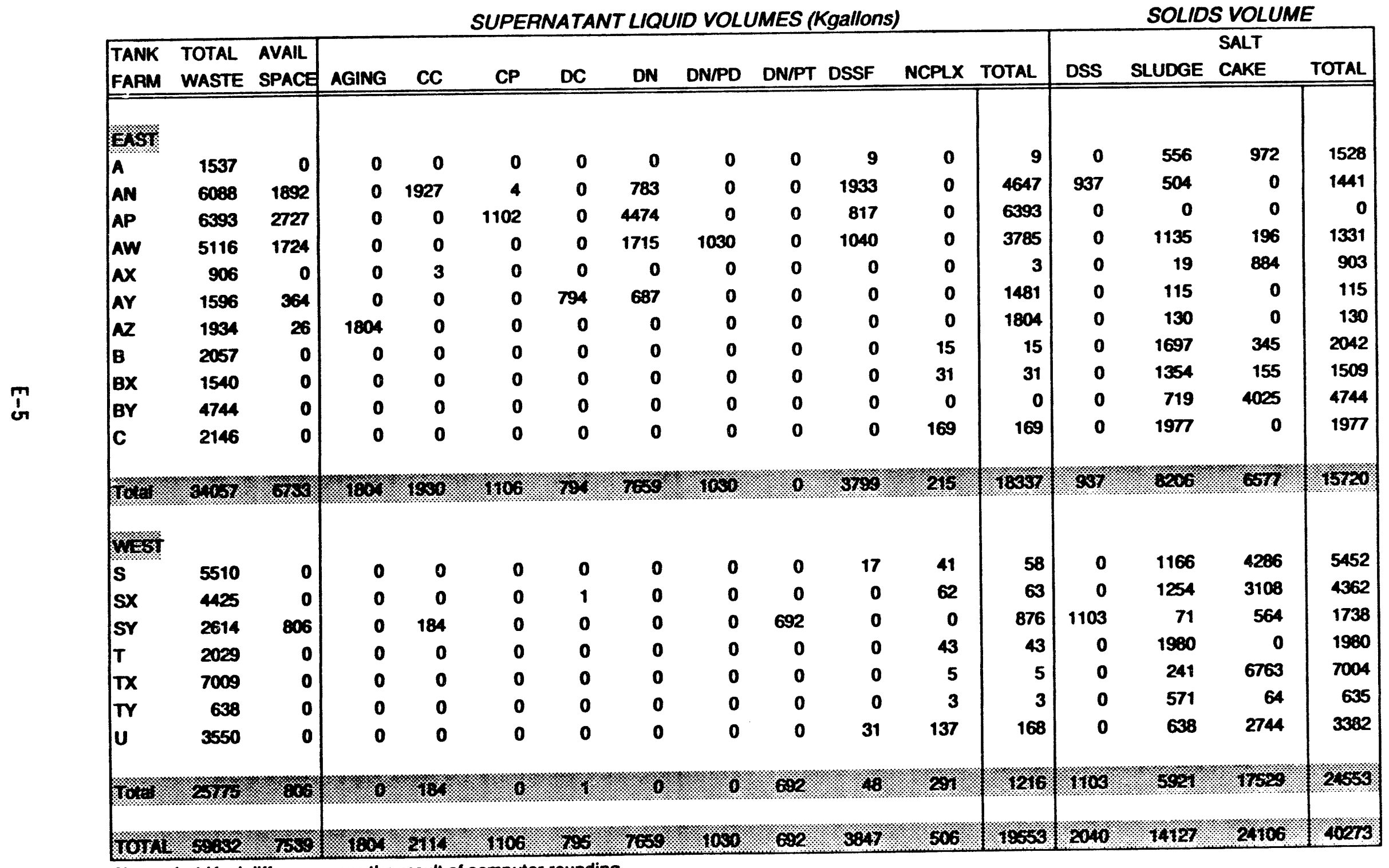


TABLE E-4. INVENTORY and status or tanK DOUBLE-SHELL TAMKS

May 31, 1994

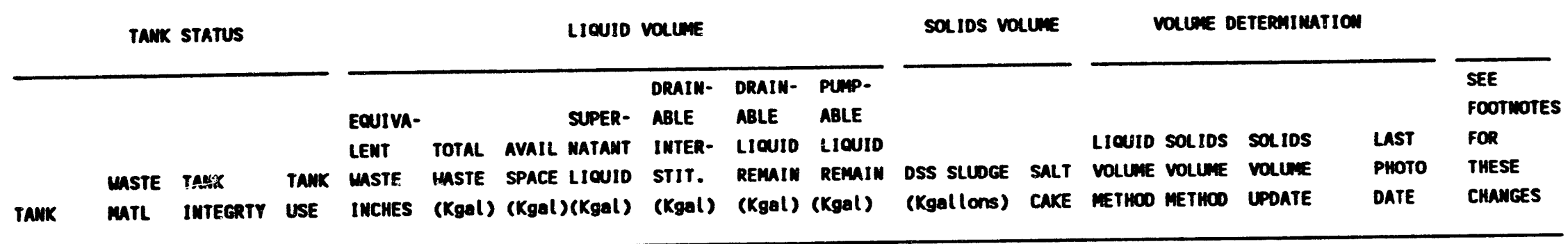

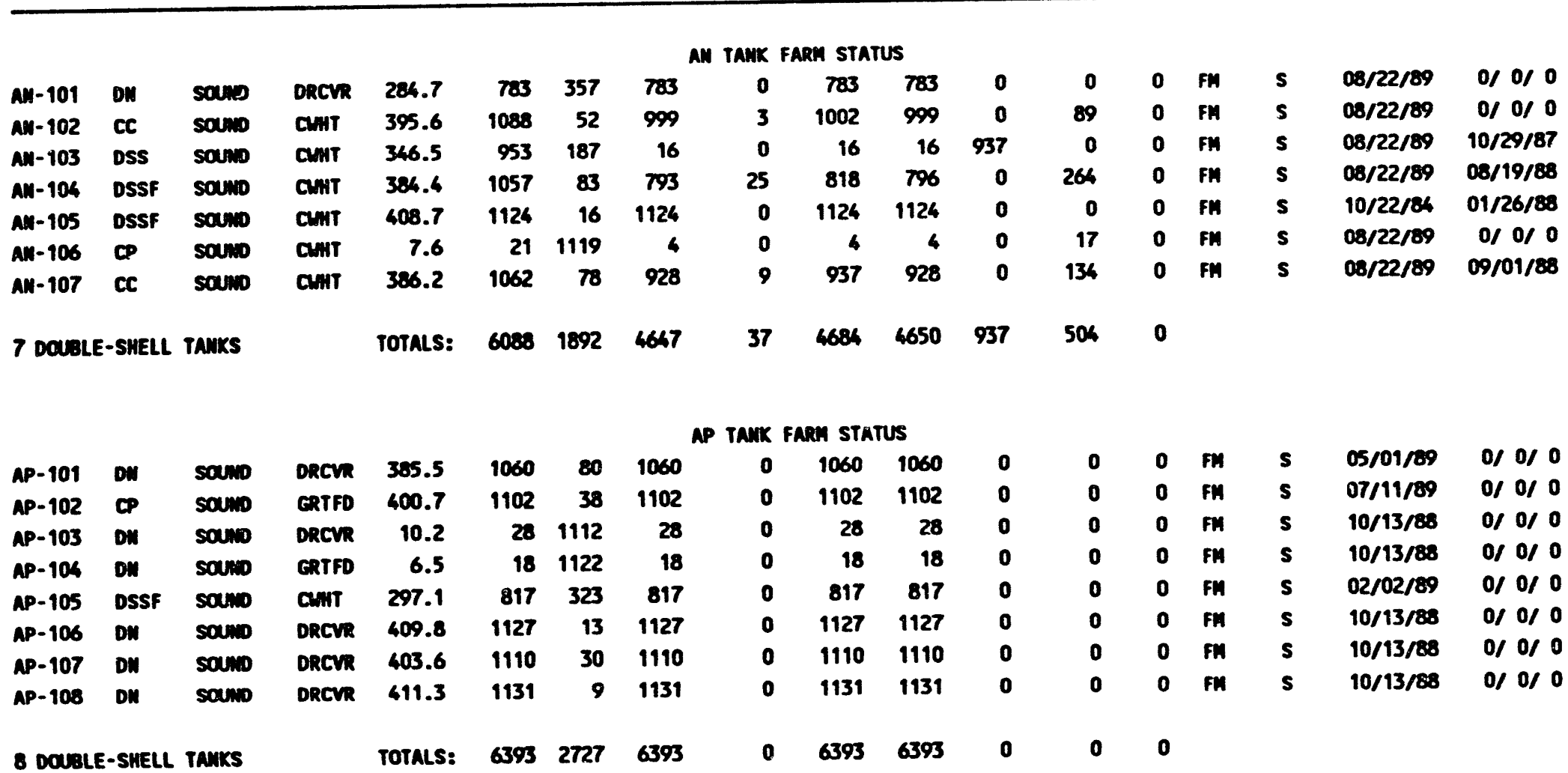


TABLE E-4. INVENTORY aNO STATUS BY TAMK

DOUBLE-SHELL TAMKS

May 31, 1994

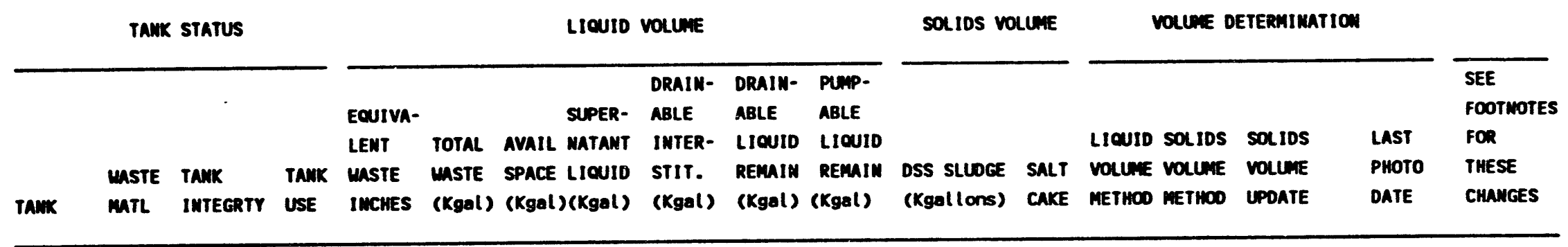

\begin{tabular}{|c|c|c|c|}
\hline \multirow{5}{*}{$\stackrel{m}{1}$} & $A W-101$ & DSSF & sound \\
\hline & $A w-102$ & DN & sound \\
\hline & $A N-103$ & DN/PO & sourd \\
\hline & $A N-104$ & & Sound \\
\hline & AW-105 & DN/PO & sourd \\
\hline & AN-106 & DN & sound \\
\hline
\end{tabular}

6 doUBLE-SHELL TANKS

$\begin{array}{lrrrr}\text { CUnT } & 408.7 & 1124 & 16 & 1040 \\ \text { EVFD } & 78.2 & 215 & 925 & 214 \\ \text { DRCVR } & 235.3 & 647 & 493 & 284 \\ \text { DRCVR } & 408.4 & 1123 & 17 & 833 \\ \text { DRCVR } & 379.3 & 1043 & 97 & 746 \\ \text { SRCVK } & 350.5 & 964 & 176 & 668 \\ & & & & \\ & \text { TOTALS: } & 5116 & 1724 & 3785\end{array}$

AW TANK FARM STATUS

$\begin{array}{rrrrrrrrrr}2 & 1042 & 1040 & \text { O } & 84 & \text { O } & \text { FH } & \text { S } & 10 / 22 / 84 & 03 / 17 / 88 \\ 0 & 214 & 214 & \text { O } & 1 & \text { O } & \text { FH } & \text { S } & 02 / 29 / 84 & 02 / 02 / 83 \\ 37 & 321 & 299 & \text { 0 } & 363 & \text { O } & \text { FH } & \text { S } & 02 / 01 / 89 & 0 / 0 / 0 \\ 49 & 882 & 860 & 0 & 179 & 111 & \text { FH } & \text { S } & 03 / 05 / 87 & 02 / 02 / 83 \\ 29 & 775 & 753 & 0 & 297 & \text { O } & \text { FH } & \text { S } & 03 / 05 / 87 & 0 / 0 / 0 \\ 42 & 710 & 688 & 0 & 211 & 85 & \text { FH } & \text { S } & 01 / 31 / 92 & 02 / 02 / 83 \\ 159 & 3944 & 3854 & 0 & 1135 & 196 & & & & \end{array}$

2 DOUBLE-SHELL TAMKS

TOTALS: $1596 \quad 364 \quad 1481$

TAMK FARM Status

\begin{tabular}{|c|c|c|c|c|c|c|c|c|c|c|c|c|c|c|c|c|c|}
\hline \multicolumn{18}{|c|}{ me ar touna ravin sinivo } \\
\hline AY-101 & DC & sound & DRCVR & 318.9 & 877 & 103 & 794 & 2 & 796 & 794 & 0 & 83 & 0 & $\mathbf{F n}$ & $\mathbf{s}$ & $02 / 02 / 87$ & $12 / 28 / 82$ \\
\hline$A Y-102$ & DN & socund & DRCVR & 261.5 & 719 & 261 & 687 & 0 & 687 & 687 & 0 & 32 & 0 & $\mathbf{F M}$ & $\mathbf{s}$ & $02 / 10 / 88$ & $04 / 28 / 81$ \\
\hline 2 DOUBL & She & anks & & TOTALS: & 1596 & 364 & 1681 & 2 & 1483 & 1481 & $\mathbf{0}$ & 115 & $\mathbf{0}$ & & & & \\
\hline
\end{tabular}

\begin{tabular}{|c|c|c|c|c|c|c|c|c|c|c|c|c|c|c|c|c|c|}
\hline & & & & & & & & AZ TAMK & RA STA & & & & & & & & \\
\hline A2-101 & AGING & soum & ant & 351.6 & 967 & 13 & 932 & 0 & 932 & 932 & 0 & 35 & 0 & Fm & $\mathbf{s}$ & $09 / 30 / 90$ & $08 / 18 / 83$ \\
\hline az-102 & AGING & sound & DRCVR & 351.6 & 967 & 13 & 872 & 4 & 876 & 872 & 0 & 95 & 0 & $\mathbf{F M}$ & $\mathbf{s}$ & $06 / 04 / 92$ & $12 / 24 / 84$ \\
\hline ma & SHEL & Tamks & & TOTALS: & 1934 & 26 & 1804 & 4 & 1808 & 1804 & 0 & 130 & o & & & & \\
\hline
\end{tabular}


TABLE E-4. INEETTOR AND STATUS or TAMK

DOUBLE-SHELL TANKS

Moy 31. 1994

\begin{tabular}{|c|c|c|c|c|c|c|c|c|c|c|c|c|c|c|c|c|c|}
\hline \multicolumn{4}{|c|}{ Tank status } & \multicolumn{7}{|c|}{ LIOUID Volune } & \multicolumn{2}{|c|}{ SOLIDS VOLUME } & \multicolumn{4}{|c|}{ Vocune DETERAnIMATION } & \multirow[b]{2}{*}{$\begin{array}{l}\text { SEE } \\
\text { FOOTMOTES } \\
\text { FOR } \\
\text { THESE } \\
\text { CHAMEES }\end{array}$} \\
\hline TAMK & $\begin{array}{l}\text { MASTE } \\
\text { MATL }\end{array}$ & $\begin{array}{l}\text { TANK } \\
\text { IMTEERTY }\end{array}$ & $\begin{array}{l}\text { TAMKK } \\
\text { USE }\end{array}$ & $\begin{array}{l}\text { EQUIVA- } \\
\text { LEMT } \\
\text { MASTE } \\
\text { INCHES }\end{array}$ & $\begin{array}{l}\text { TOTAL } \\
\text { MASTE } \\
\text { (Kgal) }\end{array}$ & $\begin{array}{l}\text { AVAIL } \\
\text { SPACE } \\
\text { (Kgal) }\end{array}$ & $\begin{array}{l}\text { SUPER- } \\
\text { MATANT } \\
\text { LIOUID } \\
\text { (Kgal) }\end{array}$ & $\begin{array}{l}\text { DRAIN- } \\
\text { ABLE } \\
\text { INTER- } \\
\text { STIT. } \\
\text { (Kgal) }\end{array}$ & $\begin{array}{l}\text { DRAIN- } \\
\text { ABLE } \\
\text { LIOUID } \\
\text { REAMIN } \\
\text { (Kgal) }\end{array}$ & $\begin{array}{l}\text { PUIP- } \\
\text { ABLE } \\
\text { LIQUID } \\
\text { REMIN } \\
\text { (Kgal) }\end{array}$ & $\begin{array}{l}\text { DSS SLUDGE } \\
\text { (Kgallons) }\end{array}$ & $\begin{array}{l}\text { SALT } \\
\text { CAKE }\end{array}$ & $\begin{array}{l}\text { Licuid } \\
\text { VOLUARE } \\
\text { METMOO }\end{array}$ & $\begin{array}{l}\text { SOLIOS } \\
\text { VOLUNE } \\
\text { METHOD }\end{array}$ & $\begin{array}{l}\text { SOLIDS } \\
\text { VOLUME } \\
\text { UPDATE }\end{array}$ & $\begin{array}{l}\text { LAST } \\
\text { PHOTO } \\
\text { DATE }\end{array}$ & \\
\hline
\end{tabular}

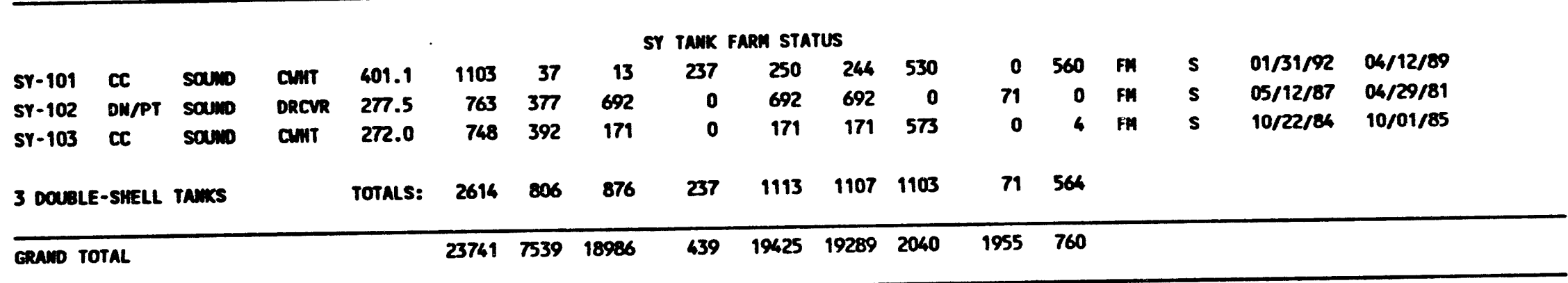

Mote: $+1-1 \mathrm{kgal}$ differences are the result of computer rounding

\begin{tabular}{|c|c|c|c|c|}
\hline \multirow[b]{2}{*}{ Tentk Farms } & \multirow{2}{*}{$\begin{array}{l}\text { Available Space } \\
\text { Calculations Used } \\
\text { In This Docunent } \\
\text { (Host Conservative) }\end{array}$} & \multicolumn{2}{|c|}{ Document SD-LA-TI-357" } & \multirow{2}{*}{$\begin{array}{l}050-T-151-00007 \\
\text { Specification Limit }\end{array}$} \\
\hline & & Operating Limit & Tank Capecity & \\
\hline $\begin{array}{l}A M, A P, A W, S Y \\
A Y, A Z \text { (Aging Weste) }\end{array}$ & $\begin{array}{r}1,140,000 \mathrm{gal}(414.5 \text { in. }) \\
900,000 \mathrm{gal}(356.4 \mathrm{in.})\end{array}$ & $\begin{array}{l}1,144,000 \text { gat (416 in.) } \\
990,000 \text { gat (360 in.) }\end{array}$ & $\begin{array}{l}1,160,000 \mathrm{gal}(421.8 \mathrm{in.}) \\
1,000,000 \mathrm{gal}(363.6 \mathrm{in.})\end{array}$ & $\begin{array}{l}1,160,500 \mathrm{gal}(422 \mathrm{in.}) \\
1,001,000 \mathrm{gal}(364 \mathrm{in.})\end{array}$ \\
\hline
\end{tabular}

\footnotetext{
- MHC-SD-MH-TI-357, Maste Storage Tank Status and Leak Detection Criteria."

muC-0SD-T-151-00007, moperating Specifications for 261-AN, AP, AM, AY, AZ, \& SY Tank Farms."
} 
TABLE E-5. IMVEMTORY AND STATUS BY TAMK

SIMGLE-SHELL TAMKS

May 31, 1996

\begin{tabular}{|c|c|c|c|c|c|c|c|c|c|c|c|c|c|c|c|c|c|c|}
\hline \multirow[b]{2}{*}{ TaMK } & \multicolumn{3}{|c|}{ TAMK STATUS } & \multicolumn{7}{|c|}{ LIOUID VolunE } & \multicolumn{2}{|c|}{ SOLIDS VOLLANE } & \multicolumn{6}{|c|}{ VOLUNE DETERNIMATION } \\
\hline & $\begin{array}{l}\text { MASTE } \\
\text { MAERIAL }\end{array}$ & $\begin{array}{l}\text { TAMK } \\
\text { INTEGRITY }\end{array}$ & $\begin{array}{l}\text { STABIL/ } \\
\text { Isolation } \\
\text { status }\end{array}$ & $\begin{array}{l}\text { TOTAL } \\
\text { UASTE } \\
\text { (Kgal) }\end{array}$ & $\begin{array}{l}\text { SUPER- } \\
\text { MATANT } \\
\text { LICUID } \\
\text { (Kgal) }\end{array}$ & $\begin{array}{l}\text { DRAIM- } \\
\text { ABLE } \\
\text { INTER- } \\
\text { STIT. } \\
\text { (Kgal) }\end{array}$ & $\begin{array}{l}\text { PUNPED } \\
\text { THIS } \\
\text { MoNTH } \\
\text { (Kgal) }\end{array}$ & $\begin{array}{l}\text { TOTAL } \\
\text { PUmped } \\
\text { (Kgal) }\end{array}$ & $\begin{array}{l}\text { DRAIN- } \\
\text { ABLE } \\
\text { LIOUID } \\
\text { REMIN } \\
\text { (Kgal) }\end{array}$ & $\begin{array}{l}\text { PUAP- } \\
\text { ABLE } \\
\text { LIOUID } \\
\text { REMAIN } \\
\text { (Kgal) }\end{array}$ & $\begin{array}{l}\text { SLWoe } \\
\text { (Kgal) }\end{array}$ & $\begin{array}{l}\text { SALtcAKE } \\
\text { (Kgal) }\end{array}$ & $\begin{array}{l}\text { Liouios } \\
\text { volune } \\
\text { netnoo }\end{array}$ & $\begin{array}{l}\text { solios } \\
\text { volune } \\
\text { netion }\end{array}$ & $\begin{array}{l}\text { Solios } \\
\text { Woure } \\
\text { vophte }\end{array}$ & $\begin{array}{l}\text { SOLIDS } \\
\text { UPOATE } \\
\text { SOURCE } \\
\text { SEE } \\
\text { FOOTWOTE }\end{array}$ & $\begin{array}{c}\text { LAST } \\
\text { PHOTO } \\
\text { DATE }\end{array}$ & $\begin{array}{l}\text { SEE } \\
\text { FOOTWOTES } \\
\text { FOR } \\
\text { TwESE } \\
\text { CunueEs }\end{array}$ \\
\hline & & & & & & +++1 & A farea $s$ & Status & ++4 & & & & & & & & & \\
\hline$A-101$ & DSsf & sound & PPI & 953 & 0 & 413 & 0.0 & 0.0 & 413 & 390 & 3 & 950 & $P$ & $\mathbf{F}$ & $11 / 21 / 80$ & & $08 / 21 / 85$ & \\
\hline$A-102$ & DSSF & soun & IS/PI & 41 & 4 & 2 & 0.0 & 39.5 & 6 & 0 & 15 & 22 & $\mathbf{P}$ & FP & $07 / 27 / 89$ & (1) & $07 / 20 / 89$ & \\
\hline$A-103$ & DSSF & Asto Lix & IS/IP & 371 & 5 & 15 & 0.0 & 111.0 & 20 & 0 & 366 & 0 & - & FP & $06 / 03 / 88$ & (1) & $12 / 28 / 88$ & \\
\hline$A-104$ & $\operatorname{mcplx}$ & ASN LER & IS/IP & 28 & 0 & 0 & 0.0 & 0.0 & 0 & 0 & 28 & 0 & $\mathbf{n}$ & PS & $01 / 27 / 78$ & & $06 / 25 / 86$ & \\
\hline$A-105$ & MCPLX & Asm LKR & $15 / 1 P$ & 19 & 0 & 4 & 0.0 & 0.0 & 4 & 0 & 19 & 0 & $\mathbf{P}$ & $\mathbf{m}$ & $08 / 23 / 79$ & (1) & $08 / 20 / 86$ & \\
\hline$A-106$ & $\mathbf{C P}$ & souno & IS/IP & 125 & 0 & 7 & 0.0 & 0.0 & 7 & 0 & 125 & 0 & $\mathbf{P}$ & $n$ & $09 / 07 / 82$ & & $08 / 17 / 86$ & \\
\hline 6 singt & LE-SHELL & Tauks & TOTALs & 1537 & 9 & 461 & 0.0 & 150.5 & 450 & 390 & 556 & 972 & & & & & & \\
\hline & & & & & & 14+4 & aX FABN & status & t+t+t & & & & & & & & & \\
\hline$N x-101$ & Dssf & sound & API & 748 & 0 & 320 & 0.0 & 0.0 & 320 & 298 & 3 & 745 & $P$ & $\mathbf{F}$ & $05 / 06 / 82$ & & $08 / 18 / 87$ & \\
\hline $2 x-102$ & cC & ASID LKR & IS/IP & 39 & 3 & 14 & 0.0 & 13.0 & 17 & 3 & 7 & 29 & $F$ & $\mathbf{s}$ & $09 / 06 / 88$ & & $06 / 05 / 89$ & \\
\hline$a x-103$ & $\boldsymbol{c c}$ & soum & $15 / 1 P$ & 112 & 0 & 36 & 0.0 & 0.0 & 36 & 3 & 2 & 110 & $\mathbf{F}$ & $\mathbf{s}$ & $08 / 19 / 87$ & & $08 / 13 / 87$ & \\
\hline$N x-104$ & $\operatorname{mcplx}$ & Asm LK & IS/IP & 7 & 0 & 0 & 0.0 & 0.0 & 0 & 0 & 7 & 0 & $\mathbf{P}$ & $n$ & $04 / 28 / 82$ & & $08 / 18 / 87$ & \\
\hline 4 siwg & LE-SHELL & Tanks & Totals: & 906 & 3 & 370 & 0.0 & 13.0 & 373 & 304 & 19 & 824 & & & & & & \\
\hline
\end{tabular}


TABLE E-5. IMENTORY AND STATUS BY tAMK SIMGLE-SHELL TAMKS

May 31, 1994

TANK STATUS

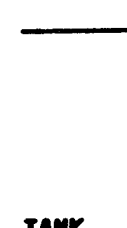
TAMK STABIL/ TOTAL MATANT INTER- THIS TOTAL LIOUID LIOUID
ISOLATION UASTE LICUID STIT. MONTH PUMPED REMIN REMUIN SUPER- ABLE PUMPED ABLE ABLE Materia IMTECoITY status (Kagl) (Kgal) (Kgal) (Kgal) (Kgal) (Kgal) (Kgal)

\begin{tabular}{|c|c|c|c|c|c|c|c|c|c|c|c|c|c|c|c|c|c|}
\hline & & & & & & ++6 & ARH ST & ITUS & & & & & & & & & \\
\hline $8-101$ & MCPLX & AST LKR & IS/IP & 113 & 0 & 6 & 0.0 & 0.0 & 6 & 0 & 113 & 0 & $\mathbf{P}$ & $\mathbf{F}$ & $0</ 28 / 82$ & & $05 / 19 / 83$ \\
\hline $8-102$ & MCPLX & sound & IS/IP & 32 & 4 & 0 & 0.0 & 0.0 & 4 & 0 & 18 & 10 & $\mathbf{P}$ & $\mathbf{F}$ & $08 / 22 / 85$ & (1) & $08 / 22 / 85$ \\
\hline B-103 & MCPLX & ASED LKR & IS/IP & 59 & 0 & 0 & 0.0 & 0.0 & 0 & 0 & 59 & 0 & F & $\mathbf{F}$ & $02 / 28 / 85$ & (1) & $10 / 13 / 88$ \\
\hline $8-104$ & MCPLX & sound & IS/IP & 371 & 1 & 46 & 0.0 & 0.0 & 47 & 40 & 301 & 69 & $n$ & $n$ & $06 / 30 / 85$ & (1) & $10 / 13 / 80$ \\
\hline $8-105$ & MCPLX & ASED LIRR & IS/IP & 306 & 0 & 23 & 0.0 & 0.0 & 23 & 0 & 40 & 266 & $P$ & $m$ & $12 / 27 / 84$ & (1) & $05 / 19 / 88$ \\
\hline e.106 & MCPLX & sound & IS/IP & 117 & 1 & 6 & 0.0 & 0.0 & 7 & 0 & 116 & 0 & $F$ & $F$ & $03 / 31 / 85$ & (1) & $02 / 28 / 85$ \\
\hline 8-107 & MCPLX & AST LIRR & IS/IP & 165 & 1 & 12 & 0.0 & 0.0 & 13 & 7 & 164 & 0 & 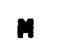 & $M$ & $03 / 31 / 85$ & (1) & $02 / 28 / 85$ \\
\hline $8-108$ & MCPLX & sound & IS/IP & 94 & 0 & 4 & 0.0 & 0.0 & 4 & 0 & 94 & 0 & $F$ & $\mathbf{F}$ & $05 / 31 / 85$ & (1) & $05 / 10 / 85$ \\
\hline $8-109$ & MCPLX & sand & IS/IP & 127 & 0 & 8 & 0.0 & 0.0 & 8 & 0 & 127 & 0 & $\mathbf{m}$ & $n$ & $06 / 08 / 85$ & (1) & $04 / 02 / 85$ \\
\hline B-110 & MCPLX & ASTO IR & IS/IP & 246 & 1 & 22 & 0.0 & 0.0 & 23 & 17 & 245 & 0 & $\mathbf{m}$ & $m$ & $02 / 28 / 85$ & (1) & $03 / 17 / 88$ \\
\hline $8-111$ & MCPLX & ASED LKR & IS/IP & 237 & 1 & 21 & 0.0 & 0.0 & 22 & 16 & 236 & 0 & F & F & $06 / 28 / 85$ & (1) & $06 / 26 / 85$ \\
\hline $8-112$ & MCPLX & ASED LKR & IS/IP & 33 & 3 & 0 & 0.0 & 0.0 & 3 & 0 & 30 & 0 & $F$ & F & $05 / 31 / 85$ & (1) & $05 / 29 / 85$ \\
\hline$B-201$ & MCPLX & ASID LKR & IS/IP & 29 & 1 & 3 & 0.0 & 0.0 & 4 & 0 & 28 & 0 & $\boldsymbol{n}$ & $\boldsymbol{n}$ & $04 / 28 / 82$ & & $11 / 12 / 86$ \\
\hline $8-202$ & MCPLX & sown & IS/IP & 27 & 0 & 3 & 0.0 & 0.0 & 3 & 0 & 27 & 0 & $p$ & $\boldsymbol{n}$ & $05 / 31 / 85$ & (1) & $05 / 29 / 85$ \\
\hline $8-203$ & MCPLX & ASPD LKR & IS/IP & 51 & 1 & 5 & 0.0 & 0.0 & 6 & 0 & 50 & 0 & PN & $\mathbf{P M}$ & $05 / 31 / 84$ & (1) & $11 / 13 / 86$ \\
\hline $8-204$ & MCPLX & ASWD LXR & IS/IP & 50 & 1 & 5 & 0.0 & 0.0 & 6 & 0 & 49 & 0 & $P$ & $n$ & $05 / 31 / 84$ & (1) & $10 / 21 / 87$ \\
\hline $16 \mathrm{sim}$ & GLE-SHEL & Tanks & Totals & 2057 & 15 & 164 & 0.0 & 0.0 & 179 & 80 & 1697 & 345 & & & & & \\
\hline
\end{tabular}


TABLE E-5. IMVENTORY AND STATUS BY TAMK SINGLE-SHELL TANKS

May 31, 1994

TANK Status

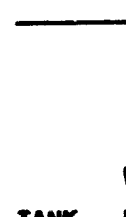

TAMT MATERIAL INTECRITY STATUS
SOLIDS VOLUEE

DRAIN- PUMP-

ABLE ABLE.

SUPER- ABLE PUMPED

STABIL/ TOTAL MATANT IMTER- THIS TOTAL LIOUID LIOUID

ISOLATION IMSTE LIOUID STIT. MONTH PUMPED REMIN REMII

$\longrightarrow$

SLLOGE SALTCACE (Kgal) (Kgal)
VOLUE DETERMIUATIOO

\begin{tabular}{|c|c|c|c|c|c|}
\hline & & & SOLIDS & & SEE \\
\hline & & & UPDATE & & FCOTWOTES \\
\hline $\begin{array}{l}\text { LIOUIOS } \\
\text { volume } \\
\text { METHOD }\end{array}$ & $\begin{array}{l}\text { SOLIOS } \\
\text { VoLUEE } \\
\text { NETHOD }\end{array}$ & $\begin{array}{l}\text { SOLIOS } \\
\text { VOLUNE } \\
\text { UPOATE }\end{array}$ & $\begin{array}{c}\text { SOURCE } \\
\text { SEE } \\
\text { Footmote }\end{array}$ & $\begin{array}{r}\text { LAST } \\
\text { PHOTO } \\
\text { DATE }\end{array}$ & $\begin{array}{l}\text { FOR } \\
\text { ? ESE } \\
\text { CMUUEES }\end{array}$ \\
\hline
\end{tabular}

\begin{tabular}{|c|c|c|c|c|c|c|c|c|c|c|c|c|c|c|c|c|c|}
\hline & & & & & +4 & FARM : & Status & $+4+4+4$ & & & & & & & & & \\
\hline 8X-101 MCPLX & ASID LKR & IS/IP & 43 & 1 & 0 & 0.0 & 0.0 & 1 & 0 & 42 & $\mathbf{0}$ & $\mathbf{P}$ & $M$ & $04 / 28 / 82$ & & $11 / 26 / 88$ & \\
\hline Bx-102 MCPLX & ASIMD LKR & IS/IP & 96 & 0 & 4 & 0.0 & 0.0 & 4 & 0 & 96 & 0 & $\mathbf{P}$ & $\mathbf{m}$ & $04 / 28 / 82$ & & $09 / 18 / 85$ & \\
\hline $8 x-103$ MCPLX & sound & IS/IP & 66 & 4 & 0 & 0.0 & 0.0 & 4 & 0 & 62 & 0 & $\mathbf{P}$ & $\mathbf{F}$ & $11 / 29 / 83$ & & $10 / 31 / 86$ & \\
\hline $8 x-106$ NCPLX & souno & IS/IP & $\varphi$ & 3 & 30 & 0.0 & 17.4 & 33 & 27 & 96 & $\mathbf{0}$ & $\mathbf{F}$ & $\mathbf{F}$ & $09 / 22 / 89$ & (1) & $09 / 21 / 89$ & \\
\hline $8 x-105$ NCPLX & soumd & IS/IP & 51 & 5 & 6 & 0.0 & 15.0 & 11 & 4 & 43 & 3 & $F$ & $\mathbf{s}$ & $09 / 03 / 86$ & (1) & $10 / 23 / 86$ & \\
\hline BX-106 MCPLX & sound & PPI & 46 & 15 & 0 & 0.0 & 0.0 & 15 & 15 & 31 & 0 & $\mathbf{m}$ & PS & $04 / 28 / 82$ & & $05 / 19 / 88$ & \\
\hline$B X-107$ MCPLX & sound & IS/PI & 345 & 1 & 29 & 0.0 & 23.1 & 30 & 23 & 344 & 0 & $\mathbf{m}$ & $\mathbf{P}$ & $09 / 18 / 90$ & (2) & $09 / 11 / 90$ & \\
\hline $8 x-108$ NCPLX & ASWD LKR & IS/IP & 26 & 0 & 1 & 0.0 & 0.0 & 1 & 0 & 26 & 0 & $n$ & PS & $07 / 31 / 79$ & (1) & $05 / 05 / 94$ & \\
\hline$B x-109$ NCPLX & soumo & IS/PI & 193 & 0 & 13 & 0.0 & 8.2 & 13 & 8 & 193 & 0 & FP & $p$ & $09 / 17 / 90$ & (2) & $09 / 11 / 90$ & \\
\hline$B X-110$ NCPLX & ASYO LKR & IS/PI & 198 & 0 & 15 & 0.0 & 4.0 & 17 & 6 & 189 & 9 & $\mathbb{P}$ & $M$ & $08 / 22 / 85$ & (1) & $07 / 31 / 65$ & \\
\hline$B X-111$ NCPLX & ASIDO LKR & $\mid P I$ & 212 & 1 & 29 & 3.3 & 110.4 & 30 & 8 & 68 & 143 & $\boldsymbol{M}$ & $M$ & $07 / 26 / 77$ & & $05 / 19 / 94$ & (a) \\
\hline$B x-112$ NCPLX & sound & IS/PI & 165 & 1 & 7 & 0.0 & 4.1 & 8 & 2 & 164 & 0 & FP & $\mathbf{P}$ & $09 / 17 / 90$ & (2) & $09 / 11 / 90$ & \\
\hline 12 SIMGLE-SHELL & Tanks & rotals: & 1540 & 31 & 134 & 3.3 & 182.2 & 167 & 93 & 1354 & 155 & & & & & & \\
\hline & & & & & ++ & FARM & Status & $t+t+t+$ & & & & & & & & & \\
\hline Br-101 nCPLX & sound & IS/IP & 387 & 0 & 5 & 0.0 & 35.8 & 5 & 0 & 109 & 278 & $\mathbf{P}$ & $\boldsymbol{n}$ & $05 / 30 / 84$ & & $09 / 19 / 89$ & \\
\hline BY-102 MCPLX & soum & /PI & 341 & 0 & 39 & 2.3 & 125.6 & 39 & 20 & 0 & 341 & $m$ & $M$ & $08 / 30 / 91$ & (2) & $09 / 11 / 87$ & (b) \\
\hline$B Y-103$ MCPLX & ASTD LKR & PPI & 400 & 0 & 160 & 0.0 & 78.5 & 160 & 137 & 5 & 395 & $m$ & $M$ & $04 / 03 / 90$ & (2) & $09 / 07 / 89$ & \\
\hline$B Y-104$ MCPLX & sound & IS/IP & 406 & 0 & 18 & 0.0 & 329.5 & 18 & 0 & 40 & 366 & $P$ & $n$ & $04 / 28 / 82$ & & $04 / 27 / 83$ & \\
\hline BY-105 NCPLX & ASID LKR & PPI & 503 & 0 & 192 & 0.0 & 0.0 & 192 & 169 & 44 & 459 & $\mathbf{P}$ & $\mathbf{m}$ & $04 / 28 / 82$ & & $07 / 11 / 86$ & \\
\hline$B Y-106$ MCPLX & ASHD LKR & /PI & 642 & 0 & 235 & 0.0 & 0.0 & 235 & 213 & 95 & 547 & $\mathbf{p}$ & $M P$ & $04 / 28 / 82$ & & $11 / 04 / 82$ & \\
\hline BY-107 NCPLX & ASID LKR & IS/IP & 266 & 0 & 25 & 0.0 & 56.4 & 25 & 0 & 60 & 206 & 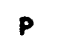 & $\mathbf{M P}$ & $04 / 28 / 82$ & & $10 / 15 / 86$ & \\
\hline
\end{tabular}



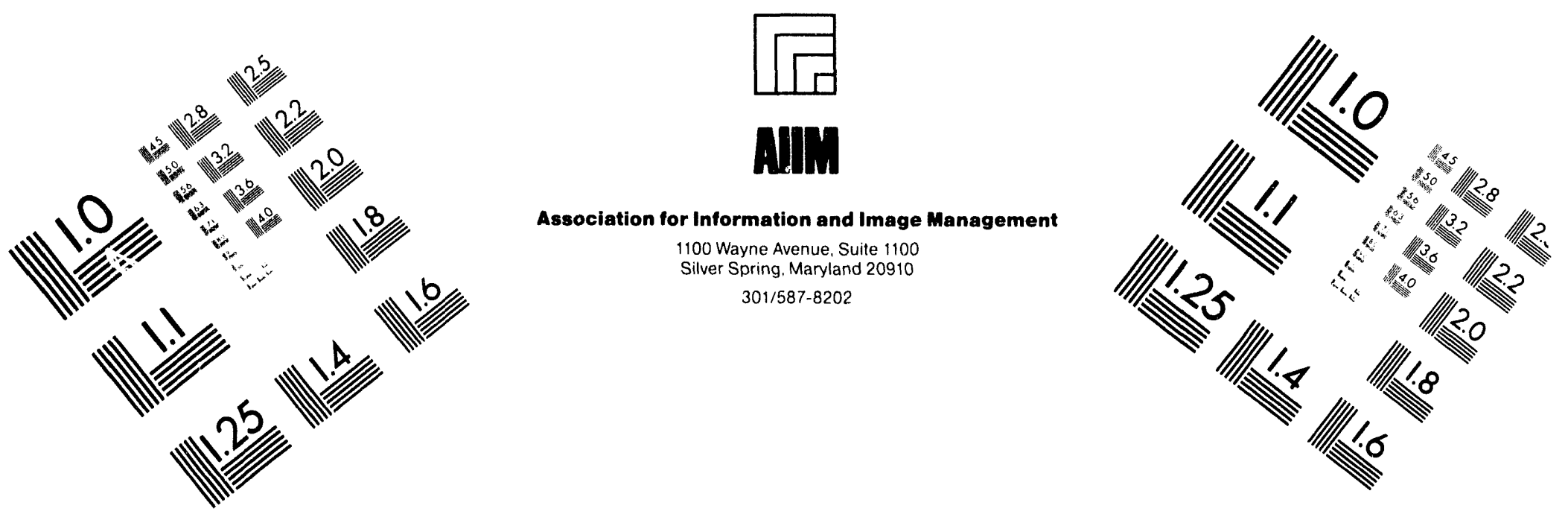

\section{Centimeter}

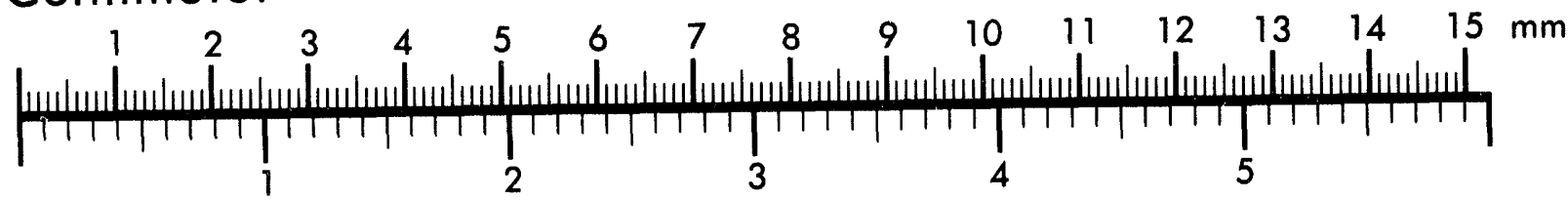
Inches
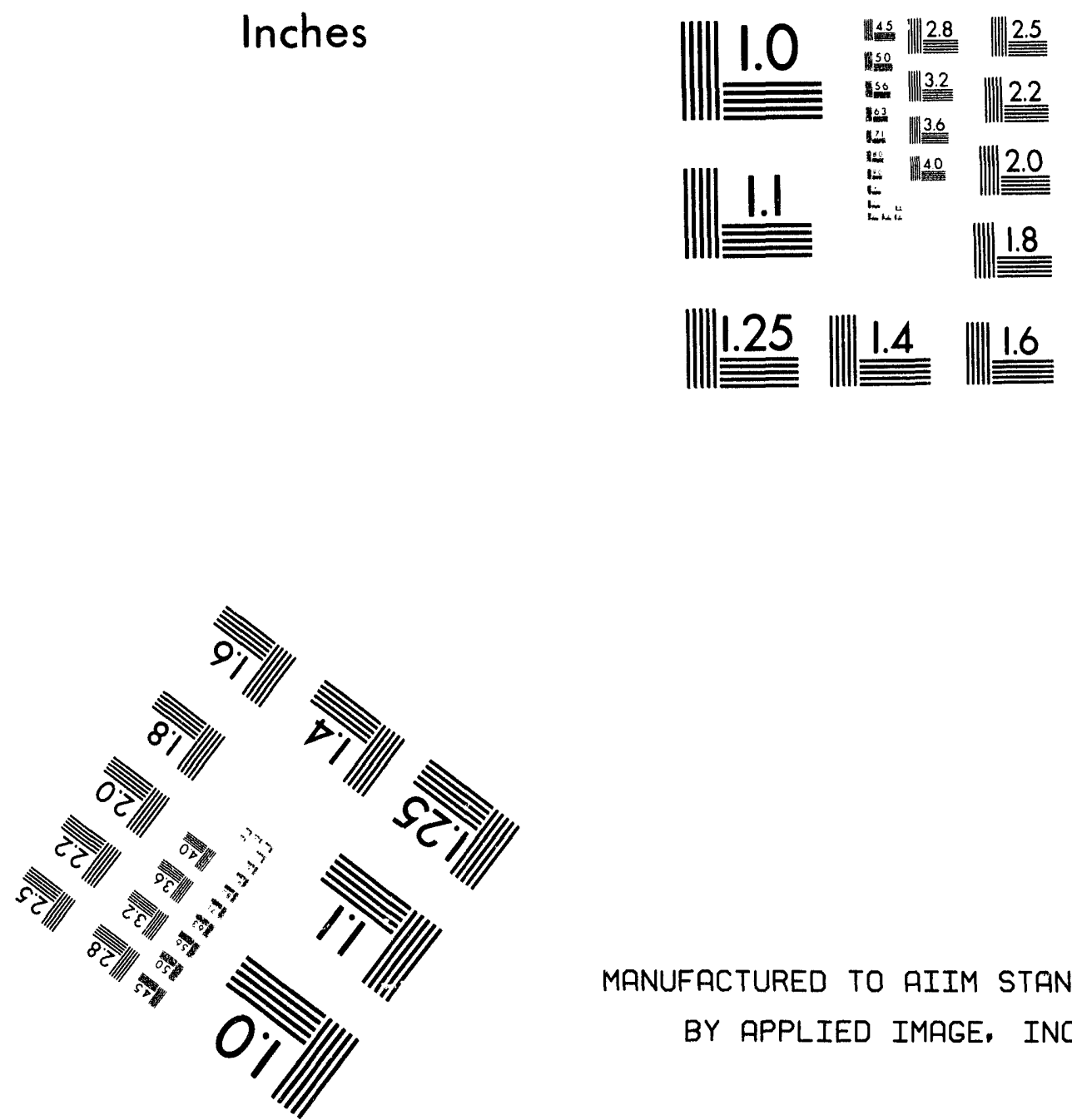

MANUFACTURED TO AIIM STANDARDS

BY APPLIED IMAGE, INC.

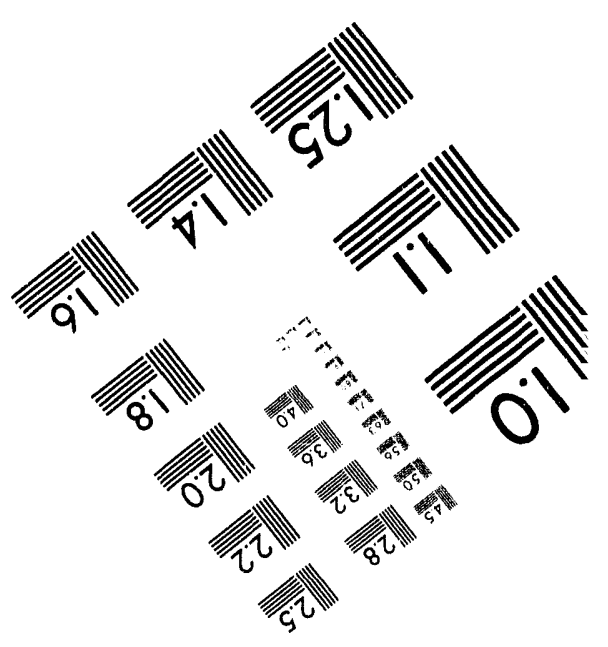



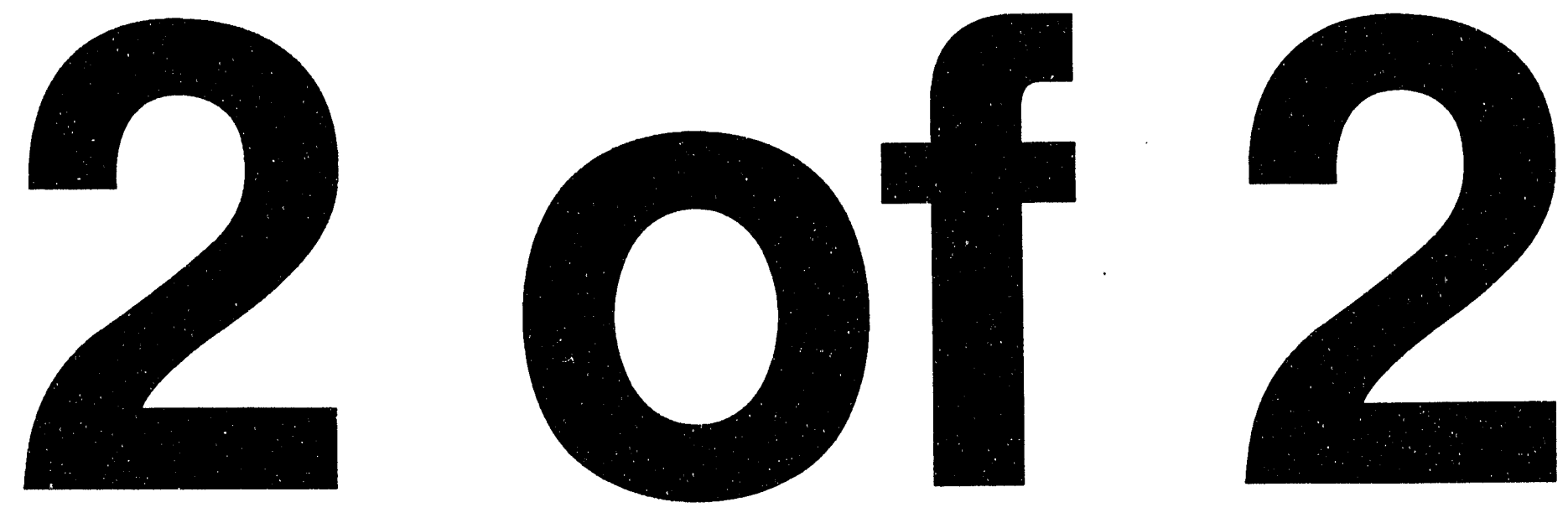
TABLE E-5. IMVEMTORY ANO STATUS BY TAMK

SINGLE-SHELL TANKS

May 31, 1994

TAMK STATUS

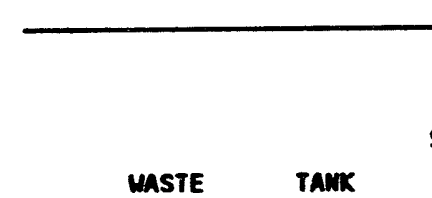

TANK MATERIAL INTEGRITY STATUS

\begin{tabular}{|c|c|c|c|}
\hline Br-108 NCPLX & ASID LKR & IS/IP & 228 \\
\hline $8 Y-109$ MCPLX & soum & IPI & 423 \\
\hline$B Y-110$ MCPLX & sowno & IS/IP & 398 \\
\hline BY-111 MCPLX & sound & IS/IP & 459 \\
\hline BY-112 NCPLX & soum & IS/IP & 291 \\
\hline 12 SIMGLE-SH & WKS & TOTALS: & 4744 \\
\hline
\end{tabular}

LIQUID VOLUNE

DRAIN- PUAP-

ABLE ABLE

STABIL/ TOTAL MATANT INTER- THIS TOTAL LIOUID LIQUIO

SOMATIO MASTE LIOUID STIT. MONTH PUMPED REMIM REMAIM

(Kgal) (Kgal) (Kgal) (Kgal) (Kgal) (Kgal) (Kgal
SOLIDS VOLUWE

$\overline{7}$

SLUDGE SALTCAKE (Kgal) (Kgal)
VOLUNE DETERAIMATION

\begin{tabular}{|c|c|c|c|c|c|}
\hline & & & SOLIDS & & SEE \\
\hline & & & UPDATE & & FCOTnOTES \\
\hline CulDS & SOLIDS & SOLIOS & SOURCE & LAST & $\begin{array}{l}\text { FOR } \\
\text { THESE }\end{array}$ \\
\hline & METHOD & UPOATE & FOOTMOTE & DATE & CHAMGES \\
\hline
\end{tabular}

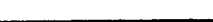

\begin{tabular}{|c|c|c|c|c|c|c|c|c|c|c|}
\hline & & & & & & & ARM 5 & atus & +t+ & \\
\hline$c-101$ & MCPLX & ASI LXR & IS/IP & 88 & 0 & 3 & 0.0 & 0.0 & 3 & 0 \\
\hline$c-102$ & DC & sourn & $\mid P \mathbf{P I}$ & 423 & 0 & 37 & 0.0 & 11.6 & 37 & 19 \\
\hline$c-103$ & MCPLX & soundio & PPI & 195 & 133 & 0 & 0.0 & 0.0 & 133 & 133 \\
\hline$C-104$ & cc & soum & IS/IP & 295 & 0 & 11 & 0.0 & 0.0 & 11 & 5 \\
\hline$c-105$ & MCPLX & soum & PPI & 150 & 0 & 11 & 0.0 & 0.0 & 11 & 4 \\
\hline$c-106$ & MCPLX & sourio & /PI & 229 & 32 & 16 & 0.0 & 0.0 & 48 & 42 \\
\hline$c-107$ & DC & sowiso & /PI & 275 & 0 & 26 & 0.0 & 16.3 & 26 & 20 \\
\hline$c-108$ & MCPLX & souno & IS/IP & 66 & 0 & 0 & 0.0 & 0.0 & $\mathbf{0}$ & 0 \\
\hline$c-109$ & MCPLX & sown & IS/IP & 66 & 4 & 0 & 0.0 & 0.0 & 4 & 0 \\
\hline$c-110$ & DC & ASIN LKR & $\mid P I$ & 187 & 0 & 7 & 0.0 & 8.9 & 7 & 5 \\
\hline$c-111$ & NCPLX & ASHD LKR & IS/IP & 57 & 0 & 0 & 0.0 & 0.0 & 0 & 0 \\
\hline$c-112$ & NCPLX & soum & IS/PI & 104 & 0 & 32 & 0.0 & 0.0 & 32 & 26 \\
\hline
\end{tabular}

$\begin{array}{rll}88 & 0 & \text { F } \\ 423 & 0 & \text { F } \\ 62 & 0 & \text { F } \\ 295 & 0 & \text { FP } \\ 150 & 0 & \text { F } \\ 197 & 0 & \text { F } \\ 275 & 0 & \text { F } \\ 66 & 0 & \text { M } \\ 62 & 0 & \text { H } \\ 187 & 0 & \text { F } \\ 57 & 0 & M \\ 104 & 0 & \text { M }\end{array}$

$\begin{array}{cc}\text { m } & 11 / 29 / 83 \\ \text { FP } & 04 / 28 / 82 \\ \text { S } & 10 / 22 / 90 \\ \text { P } & 09 / 22 / 89 \\ \text { S } & 05 / 31 / 85 \\ \text { PS } & 04 / 28 / 82 \\ \text { S } & 01 / 30 / 92 \\ \text { S } & 02 / 24 / 84 \\ \text { PS } & 11 / 29 / 83 \\ \text { FmP } & 03 / 01 / 92 \\ \text { S } & 04 / 28 / 82 \\ \text { PS } & 09 / 18 / 90\end{array}$

$11 / 17 / 87$ $05 / 18 / 76$

(2) $07 / 28 / 87$

(1) $07 / 25 / 90$

$03 / 13 / 94$

$04 / 05 / 79$

(2) $00 / 00 / 00$

(1) $12 / 05 / 74$

$01 / 30 / 76$

(2) $08 / 12 / 86$

$02 / 25 / 70$ 
TABLE E-5. INVENTORY AND STATUS BY tAMK

SINGLE-SHELL TAMKS

May 31, 1994

\begin{tabular}{|c|c|c|c|c|c|c|c|c|c|c|c|c|c|c|c|c|c|c|}
\hline \multirow[b]{2}{*}{ TAMR } & \multicolumn{3}{|c|}{ tanK status } & \multicolumn{7}{|c|}{ LIOUID VOLUAFE } & \multicolumn{2}{|c|}{ SOLIDS VOLUHE } & \multicolumn{6}{|c|}{ VOLUME DETERMIMATION } \\
\hline & $\begin{array}{l}\text { MASTE } \\
\text { MATERIAL }\end{array}$ & $\begin{array}{l}\text { TAMK } \\
\text { INTECRITY }\end{array}$ & $\begin{array}{l}\text { STABIL/ } \\
\text { ISOLATIOH } \\
\text { STATUS }\end{array}$ & $\begin{array}{c}\text { TOTAL } \\
\text { UASTE } \\
\text { (Kgal) }\end{array}$ & $\begin{array}{l}\text { SUPER- } \\
\text { MATANT } \\
\text { LICUID } \\
\text { (Kgal) }\end{array}$ & $\begin{array}{l}\text { DRAIN- } \\
\text { ABLE } \\
\text { INTER- } \\
\text { STIT. } \\
\text { (Kgal) }\end{array}$ & $\begin{array}{l}\text { PUIPED } \\
\text { THIS } \\
\text { MONTH } \\
\text { (Kgal) }\end{array}$ & $\begin{array}{l}\text { TOTAL } \\
\text { PUMPED } \\
\text { (Kgal) }\end{array}$ & $\begin{array}{l}\text { DRAIN- } \\
\text { ABLE } \\
\text { LIOUID } \\
\text { REMAIN } \\
\text { (Kgal) }\end{array}$ & $\begin{array}{l}\text { PUAP- } \\
\text { ABLE } \\
\text { LICUID } \\
\text { REMAIN } \\
\text { (Kgal) }\end{array}$ & $\begin{array}{l}\text { SLUDGE } \\
\text { (Kgal) }\end{array}$ & $\begin{array}{l}\text { SALtCAKE } \\
\text { (Kgal) }\end{array}$ & $\begin{array}{l}\text { LIOUIDS } \\
\text { Votune } \\
\text { METHod }\end{array}$ & $\begin{array}{l}\text { SOLIDS } \\
\text { YOLUWE } \\
\text { METHOD }\end{array}$ & $\begin{array}{l}\text { SOLIDS } \\
\text { VOLUIE } \\
\text { UPDATE }\end{array}$ & $\begin{array}{l}\text { SOLIDS } \\
\text { UPDATE } \\
\text { SOURCE } \\
\text { SEE } \\
\text { FOOTMOTE }\end{array}$ & $\begin{array}{l}\text { LAST } \\
\text { PHOTO } \\
\text { DATE }\end{array}$ & $\begin{array}{l}\text { SEE } \\
\text { FOOTMOTES } \\
\text { FOR } \\
\text { THESE } \\
\text { CHANGES }\end{array}$ \\
\hline$c-201$ & NCPLX & ASTD LKR & IS/IP & 2 & $\mathbf{0}$ & $\mathbf{0}$ & 0.0 & 0.0 & $\mathbf{0}$ & 0 & 2 & $\mathbf{0}$ & $\mathbf{P}$ & $\mathbf{M P}$ & $03 / 31 / 82$ & & $12 / 02 / 86$ & \\
\hline$c-202$ & EIAPTY & ASID LKR & IS/1P & 1 & $\mathbf{0}$ & $\mathbf{0}$ & 0.0 & 0.0 & $\mathbf{0}$ & 0 & 1 & $\mathbf{0}$ & $\mathbf{P}$ & $M$ & $01 / 19 / 79$ & & $12 / 09 / 86$ & \\
\hline$c-203$ & MCPLX & ASID LKR & IS/IP & 5 & 0 & $\mathbf{0}$ & 0.0 & 0.0 & $\mathbf{0}$ & $\mathbf{0}$ & 5 & $\mathbf{0}$ & $\mathbf{P}$ & MP & $04 / 28 / 82$ & & $12 / 09 / 86$ & \\
\hline$c-204$ & MCPLX & ASNO LKR & IS/IP & 3 & $\mathbf{0}$ & 0 & 0.0 & 0.0 & 0 & 0 & 3 & $\mathbf{0}$ & $\mathbf{P}$ & MP & $04 / 28 / 82$ & & $12 / 09 / 86$ & \\
\hline $16 \sin$ & GLE-SHELL & Tamiks & TOTALS: & 2146 & 169 & 143 & 0.0 & 36.8 & 312 & 254 & 1977 & 0 & & & & & & \\
\hline$s-102$ & DSSF & soumo & API & 549 & 0 & 230 & 0.0 & 0.0 & 230 & 208 & 4 & 545 & p & $\begin{array}{l}\text { PS } \\
\text { FP }\end{array}$ & $04 / 28 / 82$ & & $03 / 18 / 88$ & \\
\hline$S-103$ & DSSF & soun & PPI & 248 & 17 & 85 & 0.0 & 0.0 & 102 & 79 & 10 & 221 & n & $\mathbf{s}$ & $11 / 20 / 80$ & & $06 / 01 / 89$ & \\
\hline $5-104$ & MCPLX & ASID LKR & $1 S / 1 P$ & 294 & 1 & 28 & 0.0 & 0.0 & 29 & 23 & 293 & 0 & $M$ & $\mathbf{M}$ & $12 / 20 / 84$ & (1) & $12 / 12 / 84$ & \\
\hline s-10s & MCPLX & soum & IS/IP & 456 & 0 & 35 & 0.0 & 114.3 & 35 & 13 & 2 & 454 & $\mathbf{m P}$ & $\mathbf{s}$ & $09 / 26 / 88$ & & $04 / 12 / 89$ & \\
\hline $5 \cdot 106$ & NCPLX & sound & $\boldsymbol{M P I}$ & 479 & 4 & 186 & 0.0 & 97.0 & 190 & 168 & 28 & 447 & $P$ & EP & $12 / 31 / 93$ & & $03 / 17 / 89$ & \\
\hline s-107 & MCPLX & sound & /PI & 376 & 14 & 45 & 0.0 & 0.0 & 59 & 52 & 293 & 69 & $\mathbf{F}$ & PS & $09 / 25 / 80$ & & $03 / 12 / 87$ & \\
\hline $5-108$ & MCPLX & soum & /PI & 604 & 0 & 127 & 0.0 & 151.6 & 127 & 105 & 4 & 600 & $P$ & MP & $04 / 28 / 82$ & & $03 / 12 / 87$ & \\
\hline $5-109$ & MCPLX & sound & /PI & 568 & 0 & 141 & 0.0 & 111.0 & 141 & 119 & 13 & 555 & $\mathbf{F}$ & PS & $09 / 30 / 75$ & & $08 / 24 / 84$ & \\
\hline s-110 & MCPLXX & sound & /PI & 390 & 0 & 110 & 0.0 & 185.9 & 110 & 103 & 131 & 259 & $F$ & PS & $05 / 14 / 92$ & & $03 / 12 / 87$ & \\
\hline s-111 & NCPLX & sowno & /PI & 596 & 10 & 195 & 0.0 & 3.3 & 205 & 134 & 139 & 447 & $\mathbf{P}$ & $\mathbf{F P}$ & $04 / 28 / 82$ & & $08 / 10 / 89$ & \\
\hline s-112 & NCPLX & sown & /PI & 523 & 0 & 110 & 0.0 & 125.1 & 110 & 107 & 5 & 518 & $\mathbf{P}$ & $\mathbf{F P}$ & $12 / 31 / 93$ & & $03 / 24 / 87$ & \\
\hline $12 \mathrm{sin}$ & GLE-SHELL & Tanks & Totals: & 5510 & 58 & 1376 & 0.0 & 788.2 & 1434 & 1201 & 1166 & 4286 & & & & & & \\
\hline
\end{tabular}


table E-5. INVEATORY and status bY taMK SIMGLE-SHELL TANKS

Mar 31. 1994

\begin{tabular}{|c|c|c|c|c|c|c|c|c|c|c|c|c|c|c|c|c|c|c|}
\hline \multirow[b]{2}{*}{ TANK } & \multicolumn{3}{|c|}{ TANK Status } & \multicolumn{7}{|c|}{ LIQUID VOLUME } & \multicolumn{2}{|c|}{ SOLIDS VOLLAEE } & \multicolumn{6}{|c|}{ VOLUNE DETERMIMATION } \\
\hline & $\begin{array}{l}\text { MASTE } \\
\text { MATERIAL }\end{array}$ & $\begin{array}{l}\text { TANIK } \\
\text { INTEERITY }\end{array}$ & $\begin{array}{l}\text { STABIL/ } \\
\text { ISOLATION } \\
\text { STATUS }\end{array}$ & $\begin{array}{l}\text { TOTAL } \\
\text { UASTE } \\
\text { (Kgal) }\end{array}$ & $\begin{array}{l}\text { SUPER- } \\
\text { MATANT } \\
\text { LICUID } \\
\text { (Kgal) }\end{array}$ & $\begin{array}{l}\text { DRAIN- } \\
\text { ABLE } \\
\text { INTER- } \\
\text { STIT. } \\
\text { (Kgal) }\end{array}$ & $\begin{array}{l}\text { PUAPED } \\
\text { THIS } \\
\text { Month } \\
\text { (Kgal) }\end{array}$ & $\begin{array}{l}\text { TOTAL } \\
\text { PUAPED } \\
\text { (Kgal) }\end{array}$ & $\begin{array}{l}\text { DRAIN- } \\
\text { ABLE } \\
\text { LIOUIO } \\
\text { REMAIN } \\
\text { (Kgal) }\end{array}$ & $\begin{array}{l}\text { PUAP- } \\
\text { ABLE } \\
\text { LIOUID } \\
\text { REMAIN } \\
\text { (Kgal) }\end{array}$ & $\begin{array}{l}\text { SLUDGE } \\
\text { (Kgal) }\end{array}$ & $\begin{array}{l}\text { SALtcake } \\
\text { (Kgal) }\end{array}$ & $\begin{array}{l}\text { LICUIOS } \\
\text { VOLLEE } \\
\text { METHOD }\end{array}$ & $\begin{array}{l}\text { SOLIOS } \\
\text { VOLUME } \\
\text { METHOD }\end{array}$ & $\begin{array}{l}\text { SOLIOS } \\
\text { VOLUNE } \\
\text { UPOATE }\end{array}$ & $\begin{array}{l}\text { SOLIDS } \\
\text { UPDATE } \\
\text { SOURCE } \\
\text { SEE } \\
\text { FOOTMOTE }\end{array}$ & $\begin{array}{r}\text { LAST } \\
\text { PHOTO } \\
\text { DATE }\end{array}$ & $\begin{array}{l}\text { SEE } \\
\text { FOOTMOTES } \\
\text { FOR } \\
\text { THESE } \\
\text { CHAMGES }\end{array}$ \\
\hline $5 x-101$ & DC & sum & /P! & 456 & 1 & $\begin{array}{r}++t+t+5 \\
165\end{array}$ & $\begin{array}{c}\text { SX FARM } \\
0.0\end{array}$ & $\begin{array}{r}\text { STatus } \\
0.0\end{array}$ & $\begin{array}{r}++4+4+ \\
166\end{array}$ & 124 & 112 & 343 & D & & & & & \\
\hline $5 x-102$ & DSSF & sano & /PI & 543 & $\mathbf{0}$ & 183 & 0.0 & 0.0 & 183 & 17 & 117 & $\begin{array}{l}343 \\
426\end{array}$ & $\begin{array}{l}p \\
p\end{array}$ & $\begin{array}{l}\text { FP } \\
\boldsymbol{M}\end{array}$ & $\begin{array}{l}04 / 28 / 82 \\
04 / 28 / 82\end{array}$ & & $\begin{array}{l}03 / 10 / 89 \\
01 / 07 / 88\end{array}$ & \\
\hline $5 x-103$ & MCPLX & soum & /PI & 652 & 1 & 232 & 0.0 & 0.0 & 233 & 211 & 115 & 536 & F & $s$ & $\begin{array}{l}04 / 28 / 82 \\
07 / 15 / 91\end{array}$ & & $\begin{array}{l}01 / 07 / 88 \\
12 / 17 / 87\end{array}$ & \\
\hline $5 x-104$ & DSsf & Asm LXR & PPI & 614 & 0 & 201 & 0.0 & 113.2 & 201 & 195 & 136 & 478 & $\mathbf{F}$ & $\mathbf{s}$ & $07 / 07 / 89$ & & $09 / 08 / 88$ & \\
\hline $5 x-105$ & DSSF & soum & PPI & 683 & $\mathbf{0}$ & 261 & 0.0 & 0.0 & 261 & 238 & 73 & 610 & $\mathbf{p}$ & $\mathbf{F}$ & $04 / 28 / 82$ & & $06 / 15 / 88$ & \\
\hline $5 x-106$ & MCPLX & soumo & API & 538 & 61 & 194 & 0.0 & 0.0 & 255 & 233 & 12 & 465 & $F$ & PS & $10 / 28 / 80$ & & $06 / 01 / 89$ & \\
\hline $5 x-107$ & MCPLX & ASID LKR & IS/IP & 104 & 0 & 5 & 0.0 & 0.0 & 5 & o & 104 & 0 & $\mathbf{p}$ & $m$ & $04 / 28 / 82$ & & $03 / 06 / 87$ & \\
\hline $5 x-108$ & MCPLX & Asid IKR & IS/IP & 87 & 0 & 5 & 0.0 & 0.0 & 5 & 0 & 87 & 0 & $\mathbf{p}$ & $M$ & $12 / 31 / 93$ & & $03 / 06 / 87$ & \\
\hline $5 x-109$ & MCPLX & ASND LKR & IS/IP & 250 & 0 & 10 & 0.0 & 0.0 & 10 & 0 & 0 & 250 & P & $n$ & $10 / 05 / 93$ & & $05 / 21 / 86$ & \\
\hline $5 x-110$ & MCPLXX & ASND LKR & IS/IP & 62 & 0 & 0 & 0.0 & 0.0 & 0 & 0 & 62 & 0 & $\mathbf{n}$ & PS & $10 / 06 / 76$ & & $02 / 20 / 87$ & \\
\hline $5 x-111$ & MCPLX & ASTO LKR & IS/IP & 125 & 0 & 7 & 0.0 & 0.0 & 7 & 0 & 125 & 0 & $n$ & PS & $05 / 31 / 76$ & & $03 / 10 / 87$ & \\
\hline $5 x-112$ & MCPLX & ASHD LKR & IS/IP & 92 & 0 & 3 & 0.0 & 0.0 & 3 & 0 & 92 & 0 & $P$ & $\boldsymbol{n}$ & $04 / 28 / 82$ & & $03 / 10 / 87$ & \\
\hline $5 x-113$ & MCPLX & ASND LKR & IS/IP & 26 & 0 & 0 & 0.0 & 0.0 & 0 & 0 & 26 & 0 & $\mathbf{P}$ & $n$ & $04 / 28 / 82$ & & $03 / 18 / 88$ & \\
\hline $5 x-114$ & NCPLX & ASSD LKR & IS/IP & 181 & 0 & 14 & 0.0 & 0.0 & 16 & 0 & 181 & 0 & $p$ & H & $04 / 28 / 82$ & & $02 / 26 / 87$ & \\
\hline $5 x-115$ & NCPLX & ASID LKR & IS/IP & 12 & 0 & 0 & 0.0 & 0.0 & 0 & 0 & 12 & 0 & $P$ & $M$ & $04 / 28 / 82$ & & $03 / 31 / 88$ & \\
\hline $15 \mathrm{sim}$ & IGLE-SHELL & TAMKS & TotAls: & 4425 & 63 & 1260 & 0.0 & 113.2 & 1323 & 1178 & 1254 & 3108 & & & & & & \\
\hline
\end{tabular}


TABLE E-5. IMVETTORY AMO STATUS BY TAMK SIMGLE-SHELL TAMKS

May 31, 1994

TAMK STATUS

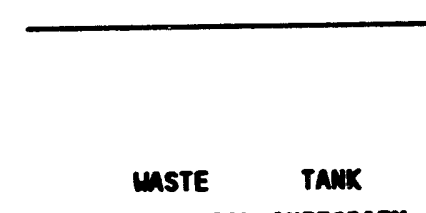

TAMR MTERIAL INTEGRITY STATUS

\begin{tabular}{|c|c|c|c|}
\hline T-101 & WCPLX & ASSID LKR & IS/PI \\
\hline$T-102$ & MCPLX & sound & IS/IP \\
\hline$T-103$ & NCPLX & ASND LKR & $I S / 1 P$ \\
\hline$T-104$ & MCPLX & soum & API \\
\hline$T-105$ & MCPLx & soum & IS/IP \\
\hline$T-106$ & MCPLX & ASTD LKR & IS/IP \\
\hline$T-107$ & MCPLX & ASID LKR & $M I$ \\
\hline$T-108$ & MCPLX & ASID LKR & IS/IP \\
\hline$T-109$ & MCPLX & ASHD LXR & IS/IP \\
\hline$T-110$ & MCPLX & soum & /P1 \\
\hline$T-111$ & EcPLx & ASTD LKR & /PI \\
\hline$T \cdot 112$ & mCPLX & soum & IS/IP \\
\hline$T-201$ & MCPLX & sould & IS/IP \\
\hline$T-202$ & MCPLX & soum & IS/IP \\
\hline$T-203$ & NCPLX & soum & IS/IP \\
\hline$T-204$ & NCPLX & sound & IS/IP \\
\hline
\end{tabular}

16 SIMGLE-SHELL TAMKS TOTALS: 2029
LIOUID VOLUME

DRAIN- PUMP-

SUPER- ABLE PUMPED

ABLE ABLE

STABIL/ TOTAL MATANT INTER- THIS TOTAL LIQUID LIQUID

UASTE LICUID STIT. MONTH PUIPED REMIN REMAIN

Kgal) (Kgal) (Kgal) (Kgal) (Kgal) (Kgal) (Kgal)
SOLIDS VOLUME

$\overline{7}$

SLWOGE SALTCAKE (Kgal) (Kgal)
VOLUNE DETERHIMATION

\begin{tabular}{lcccl}
\hline & & SOLIDS & & SEE \\
UPOATE & & FOOTMOTES \\
LIOUIDS SOLIDS & SOLIDS & SOURCE & LAST & FOR \\
VOLUAE VOLUNE & VOLUHE & SEE & PHOTO & THESE \\
METHOD METHOD & UPDATE FOOTNOTE & DATE & CHAMGES
\end{tabular}


tABLE E-5. INVENTORY AMd STATUS BY tAMK SINGLE-SHELL TAMKS

May 31, 1994

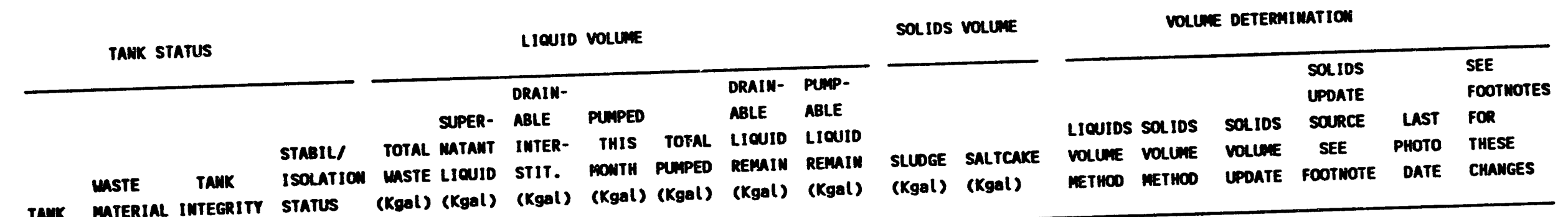

TANK MTERIAL INTEGRITY STATUS (Kgal) (Kgal) (Kgol) (Kgal) (Kgal) (Kgal)

\begin{tabular}{|c|c|c|c|c|c|c|c|c|c|c|c|c|c|c|c|c|}
\hline & & & & & $+\pi$ & $\begin{array}{r}\text { FARA } \\
0.0\end{array}$ & $\begin{array}{r}\text { status } \\
0.0\end{array}$ & 5 & 0 & 84 & 0 & $\mathbf{F}$ & $\mathbf{P}$ & $02 / 02 / 84$ & (1) & $10 / 24 / 85$ \\
\hline $\begin{array}{l}T X-101 \text { NCPLX } \\
T x-102 \text { NCPLX }\end{array}$ & $\begin{array}{l}\text { sovind } \\
\text { sovind }\end{array}$ & $\begin{array}{l}\text { IS/IP } \\
\text { IS/IP }\end{array}$ & $\begin{array}{r}87 \\
217\end{array}$ & $\begin{array}{l}3 \\
0\end{array}$ & $\begin{array}{r}2 \\
22\end{array}$ & $\begin{array}{l}0.0 \\
0.0\end{array}$ & $\begin{array}{r}0.0 \\
94.4\end{array}$ & 22 & 0 & 0 & 217 & $M$ & $\mathbf{s}$ & $08 / 31 / 84$ & & $10 / 31 / 85$ \\
\hline $\begin{array}{l}T x-102 \text { мCPLX } \\
T x-103 \text { nCPLX }\end{array}$ & $\begin{array}{l}\text { sovid } \\
\text { sowno }\end{array}$ & $\begin{array}{l}\text { IS/IP } \\
\text { IS/IP }\end{array}$ & $\begin{array}{l}218 \\
157\end{array}$ & 0 & 15 & 0.0 & 68.3 & 15 & 0 & 157 & 0 & $\mathbf{F}$ & $\mathbf{s}$ & $08 / 14 / 80$ & & $10 / 31 / 85$ \\
\hline $\begin{array}{l}T X-103 \text { NCPLX } \\
T X-104 \text { NCPLX }\end{array}$ & soum & IS/IP & 65 & 1 & 14 & 0.0 & 3.6 & 15 & 0 & 0 & 64 & $F$ & FP & $04 / 06 / 84$ & & $10 / 16 / 84$ \\
\hline TX-105 NCPLX & ASID LKR & IS/IP & 609 & 0 & 20 & 0.0 & 121.5 & 20 & 0 & 0 & 609 & $\boldsymbol{M}$ & PS & $08 / 22 / \pi$ & & $10 / 24 / 89$ \\
\hline & soumb & IS/IP & 453 & 0 & 10 & 0.0 & 134.6 & 10 & 0 & 0 & 453 & $\boldsymbol{n}$ & $\mathbf{s}$ & $08 / 29 / 77$ & & $10 / 31 / 85$ \\
\hline$T x-107$ NCPLX & ASID LKR & IS/IP & 36 & 1 & 1 & 0.0 & 0.0 & 2 & 0 & 0 & 35 & FP & FP & $01 / 20 / 84$ & (1) & $10 / 31 / 85$ \\
\hline$T X-108$ NCPLX & sound & IS/IP & 134 & 0 & 0 & 0.0 & 13.7 & 0 & 0 & 0 & 134 & $\mathbf{P}$ & FP & $05 / 30 / 83$ & & $09 / 12 / 89$ \\
\hline$T X-109$ NCPLX & sousp & $15 / 1 P$ & 384 & 0 & 10 & 0.0 & 72.3 & 10 & 0 & 0 & 384 & $\mathbf{F}$ & PS & $05 / 30 / 83$ & & $10 / 24 / 89$ \\
\hline TX-110 NCPLX & ASTD LIRR & IS/IP & 462 & 0 & 15 & 0.0 & 115.1 & 15 & 0 & 0 & 462 & $\mathbf{n}$ & PS & $05 / 30 / 83$ & & $10 / 24 / 89$ \\
\hline$T X-111$ MCPLX & sound & $15 / 19$ & 370 & 0 & 9 & 0.0 & 98.4 & 9 & 0 & $\mathbf{0}$ & 370 & $\mathbf{M}$ & PS & $07 / 26 / 77$ & & $09 / 12 / 89$ \\
\hline TX-112 мсPLX & sound & IS/IP & 649 & 0 & 24 & 0.0 & 94.0 & 24 & 0 & $\mathbf{0}$ & 649 & $\mathbf{P}$ & PS & $05 / 30 / 83$ & & $11 / 19 / 87$ \\
\hline $7 x-113$ NCPLX & ASSD LKR & IS/IP & 607 & 0 & 16 & 0.0 & 19.2 & 16 & 0 & 0 & 607 & $\mathbf{n}$ & PS & $05 / 30 / 83$ & & $04 / 11 / 83$ \\
\hline$T X-114$ NCPLX & ASTO LKR & $15 / 1 P$ & 535 & 0 & 15 & 0.0 & 104.3 & 15 & 0 & 0 & 535 & $\mathbf{M}$ & PS & $05 / 30 / 83$ & & $04 / 11 / 83$ \\
\hline$T x-115$ NCPLX & ASND LKR & IS/IP & 640 & 0 & 19 & 0.0 & 99.1 & 19 & 0 & 0 & 640 & $\mathbf{n}$ & $\mathbf{s}$ & $03 / 25 / 83$ & & $06 / 15 / 88$ \\
\hline$T X-116$ MCPLX & Asid LKR & IS/IP & 631 & 0 & 23 & 0.0 & 23.8 & 23 & $\mathbf{0}$ & 0 & 631 & $\mathbf{M}$ & PS & $03 / 31 / 72$ & & $10 / 17 / 89$ \\
\hline TX-117 nCPLX & ASED LKR & IS/IP & 626 & 0 & 8 & 0.0 & 54.3 & 8 & 0 & 0 & 626 & $n$ & PS & $12 / 31 / 71$ & & $04 / 11 / 83$ \\
\hline$T X-118$ NCPLX & sound & IS/IP & 347 & o & 27 & 0.0 & 89.1 & 27 & 0 & 0 & 347 & F & $\mathbf{s}$ & $11 / 17 / 80$ & & $12 / 19 / 79$ \\
\hline 18 SIWGLE-SHEL & TANKS & TOTALS: & 7009 & 5 & 250 & 0.0 & 1205.7 & 255 & 0 & 241 & 6763 & & & & & \\
\hline
\end{tabular}


TABLE E-5. IMVENTORY AND STATUS BY TAMK

SIMGLE-SHELL TANKS

May 31, 1994

\begin{tabular}{|c|c|c|c|c|c|c|c|c|c|c|c|c|c|c|c|c|c|c|}
\hline \multicolumn{4}{|c|}{ tank status } & \multicolumn{7}{|c|}{ LICUID volune } & \multicolumn{2}{|c|}{ SOLIDS VOLUNE } & \multicolumn{5}{|c|}{ VOLUNE DETERMIMATION } & \multirow[b]{2}{*}{$\begin{array}{l}\text { SEE } \\
\text { FOOTMOTES } \\
\text { FOR } \\
\text { THESE } \\
\text { CHANGES }\end{array}$} \\
\hline TAMx & $\begin{array}{l}\text { MASTE } \\
\text { MATERIAL }\end{array}$ & $\begin{array}{l}\text { TAMK } \\
\text { INTEGRITY }\end{array}$ & $\begin{array}{l}\text { STABIL/ } \\
\text { I SOLATIOH } \\
\text { STATUS }\end{array}$ & $\begin{array}{l}\text { TOTAL } \\
\text { MAste } \\
\text { (Kgal) }\end{array}$ & $\begin{array}{l}\text { SUPER- } \\
\text { MATANT } \\
\text { LICUID } \\
\text { (Kgal) }\end{array}$ & $\begin{array}{l}\text { DRAIN- } \\
\text { ABLE } \\
\text { INTER- } \\
\text { STIT. } \\
\text { (Kgal) }\end{array}$ & $\begin{array}{l}\text { PUIMPED } \\
\text { THIS } \\
\text { HOWTH } \\
\text { (Kgal) }\end{array}$ & $\begin{array}{l}\text { TOTAL } \\
\text { PUAPED } \\
\text { (Kgol) }\end{array}$ & $\begin{array}{l}\text { DRAIN- } \\
\text { ABLE } \\
\text { LIOUID } \\
\text { REMAIN } \\
\text { (Kgal) }\end{array}$ & $\begin{array}{l}\text { PUMP- } \\
\text { ABLE } \\
\text { LIQUID } \\
\text { REMAIN } \\
\text { (Kgal) }\end{array}$ & $\begin{array}{l}\text { SLUDEE } \\
\text { (Kgal) }\end{array}$ & $\begin{array}{l}\text { SALTCAKE } \\
\text { (Kgal) }\end{array}$ & $\begin{array}{l}\text { LIouios } \\
\text { volune } \\
\text { nethos }\end{array}$ & $\begin{array}{l}\text { SOLIOS } \\
\text { WOLUNE } \\
\text { METHOD }\end{array}$ & $\begin{array}{l}\text { SOLIDS } \\
\text { VOLLIE } \\
\text { UPOATE }\end{array}$ & $\begin{array}{l}\text { SOLIDS } \\
\text { UPDATE } \\
\text { SOURCE } \\
\text { SEE } \\
\text { FOOTWOTE }\end{array}$ & $\begin{array}{r}\text { LAST } \\
\text { PHOTO } \\
\text { DATE }\end{array}$ & \\
\hline
\end{tabular}

\begin{tabular}{|c|c|c|c|c|c|c|c|c|c|c|c|c|c|c|c|c|c|}
\hline & & & & & & ++1 & FARM & TATUS & ++++ & & & & & & & & \\
\hline$T Y-101$ & MCPLX & Asid LIR & IS/IP & 118 & 0 & 0 & 0.0 & 8.2 & 0 & 0 & 118 & 0 & $\mathbf{P}$ & $\mathbf{F}$ & $04 / 28 / 82$ & & $08 / 22 / 89$ \\
\hline$T \gamma-102$ & MCPLX & sound & IS/IP & 64 & 0 & 14 & 0.0 & 6.6 & 14 & 0 & 0 & 64 & $\mathbf{P}$ & FP & $06 / 28 / 82$ & & $07 / 07 / 87$ \\
\hline$T \gamma-103$ & MCPLX & ASTO LKR & IS/IP & 162 & 0 & 5 & 0.0 & 19.5 & 5 & 0 & 162 & 0 & $\mathbf{P}$ & FP & $07 / 09 / 82$ & & $08 / 22 / 89$ \\
\hline$T r-104$ & MCPLX & Asto LKR & IS/IP & 46 & 3 & 12 & 0.0 & 0.0 & 15 & 0 & 43 & 0 & $\mathbf{P}$ & FP & $06 / 27 / 90$ & (1) & $11 / 03 / 87$ \\
\hline$T r-105$ & MCPLX & ASm LKR & IS/IP & 231 & 0 & 0 & 0.0 & 3.6 & 0 & 0 & 231 & 0 & $\mathbf{P}$ & $n$ & $04 / 28 / 82$ & & $09 / 07 / 89$ \\
\hline$T r-106$ & NCPLX & ASSO LKR & IS/IP & 17 & 0 & 0 & 0.0 & 0.0 & 0 & 0 & 17 & 0 & $\mathbf{P}$ & $M$ & $04 / 28 / 82$ & & $08 / 22 / 89$ \\
\hline 6 SING & LE-SHELL & tamks & TOTALS: & 638 & 3 & 31 & 0.0 & 29.9 & 34 & 0 & 571 & 64 & & & & & \\
\hline & & & & & & +4 & FARM $S$ & ATUS & & & & & & & & & \\
\hline$U-101$ & NCPLX & ASm LKR & IS/1P & 25 & 3 & 0 & 0.0 & 0.0 & 3 & 0 & 22 & 0 & $\mathbf{P}$ & $\mathbf{P}$ & $04 / 28 / 82$ & & $06 / 19 / 79$ \\
\hline U-102 & MCPLX & soun & /PI & 374 & 18 & 126 & 0.0 & 0.0 & 164 & 122 & 43 & 313 & $P$ & $\operatorname{mip}$ & $04 / 28 / 82$ & & $06 / 08 / 89$ \\
\hline U-103 & MCPLX & souno & PI & 468 & 13 & 176 & 0.0 & 0.0 & 189 & 166 & 32 & 423 & $p$ & FP & $04 / 28 / 82$ & & $09 / 13 / 88$ \\
\hline$U-104$ & MCPLX & ASWD IKR & IS/IP & 122 & 0 & 7 & 0.0 & 0.0 & 7 & 0 & 122 & 0 & $P$ & MP & $04 / 28 / 82$ & & $08 / 10 / 89$ \\
\hline$U-105$ & MCPLX & soum & API & 418 & 37 & 142 & 0.0 & 0.0 & 179 & 157 & 32 & 349 & $\mathbf{F M}$ & PS & $09 / 30 / 78$ & & $07 / 07 / 88$ \\
\hline$U-106$ & McPlx & sovino & PI & 226 & 15 & 68 & 0.0 & 0.0 & 83 & 61 & 26 & 185 & $\mathbf{F}$ & PS & $12 / 30 / 83$ & & $07 / 07 / 88$ \\
\hline U-107 & DSSF & sound & PI & 406 & 31 & 147 & 0.0 & 0.0 & 178 & 156 & 15 & 360 & F & $\mathbf{s}$ & $12 / 30 / 83$ & & $10 / 27 / 88$ \\
\hline$U-108$ & MCPLX & sound & PII & 468 & 26 & 172 & 0.0 & 0.0 & 196 & 174 & 29 & 415 & F & $\mathbf{s}$ & $12 / 30 / 83$ & & $09 / 12 / 84$ \\
\hline$U-109$ & MCPLX & soum & /PI & 463 & 19 & 163 & 0.0 & 0.0 & 182 & 160 & 48 & 396 & $\mathbf{F}$ & $F$ & $11 / 13 / 77$ & & $07 / 07 / 88$ \\
\hline$U-110$ & MCPLX & ASin LR & IS/PI & 186 & 0 & 15 & 0.0 & 0.0 & 15 & 9 & 186 & 0 & $n$ & $\mathbf{m}$ & $12 / 30 / 84$ & (1) & $12 / 11 / 84$ \\
\hline$U-111$ & DSSF & soump & MPI & 329 & 0 & 122 & 0.0 & 0.0 & 122 & 99 & 26 & 303 & PS & FPS & $04 / 28 / 82$ & & $06 / 23 / 88$ \\
\hline$U-112$ & MCPLX & ASID LKR & $15 / 1 P$ & 49 & 4 & 0 & 0.0 & 0.0 & 4 & 0 & 45 & 0 & $\mathbf{P}$ & $\operatorname{mp}$ & $02 / 10 / 84$ & (1) & $08 / 03 / 89$ \\
\hline
\end{tabular}


TABLE E-5. INVENTORY AND STATUS BY TANK

SINGI E-SHELL TANKS

May 31, 1996

TANK status

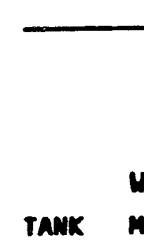

\begin{tabular}{llllllllll}
\hline U-201 NCPLX SOMN & IS/IP & 5 & 1 & 0 & 0.0 & 0.0 & 1 & 0
\end{tabular}

U-202 NCPLX SOUnd

U-203 NCPLX

IS/IP

LIOUID VOLLAE

SOLIDS VOLUME

U-204 NCPLX SOUnD

IS/IP

$\begin{array}{llll}0 & 0.0 & 0.0 & 1 \\ 0 & 0.0 & 0.0 & 1\end{array}$

T16 SIMGLE-SHELL TAMKS

Totals:

1

GRAm TOTaL

5
3
3

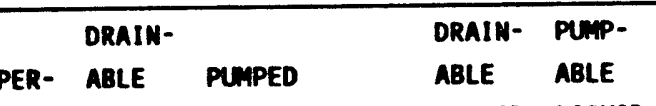

$\longrightarrow$

\begin{tabular}{|c|c|c|c|c|c|}
\hline & & & SOLIDS & & SEE \\
\hline & & & UPDATE & & FOOTMOTES \\
\hline $\begin{array}{l}\text { LICUIDS } \\
\text { VOLUME } \\
\text { METHOD }\end{array}$ & $\begin{array}{l}\text { SOL IDS } \\
\text { VOLUAE } \\
\text { METHOD }\end{array}$ & $\begin{array}{l}\text { SOLIDS } \\
\text { VOLUAE } \\
\text { UPOATE }\end{array}$ & $\begin{array}{c}\text { SOURCE } \\
\text { SEE } \\
\text { FOOTNOTE }\end{array}$ & $\begin{array}{r}\text { LAST } \\
\text { PHOTO } \\
\text { DATE }\end{array}$ & $\begin{array}{l}\text { FOR } \\
\text { THESE } \\
\text { CHAHCES }\end{array}$ \\
\hline
\end{tabular}

VOLUAE DETERMINATION

NOTES: +1- IK gal differences are the result of rounding

Total Waste is calculated as the an of sludge and Saltcake plus Supernate.

The category "Interim Isolated" (II) was changed to "Intrusion Prevention" (IP) in June 1993. See section C. "Tenk and Equipment Code and

Status Definitions"

(1) MHC-SD-RE-TI-17B SST STABILIZATION RECORO, Latest revision

(2) TANK FARHS COGWIZANT ENGIMEER MOWTHLY INPUT (Retained $10 \mathrm{yr}$ in Monthiy Sumary Report author's office)

See next page for footnotes 
TABLE E-5. INVEMTORY AMD STATUS BY TANK

SIMGLE-SHELL TANKS

May 31, 1994

TMNK STATUS

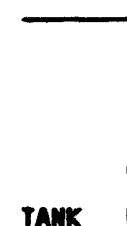

TANK MTERIAL IMTEGEITY STATUS

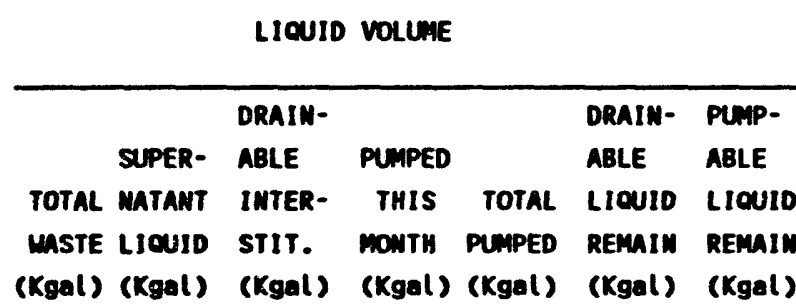

SOLIDS VOLUME

\begin{tabular}{|c|c|c|c|c|c|c|c|}
\hline & & & & & SOLIDS & & SEE \\
\hline & & & & & UPDATE & & FOOTWOTES \\
\hline $\begin{array}{l}\text { SLLDGE } \\
\text { (Kgal) }\end{array}$ & $\begin{array}{l}\text { SALTTCAKE } \\
\text { (Kgal) }\end{array}$ & $\begin{array}{l}\text { LIOUIDS } \\
\text { VOLUIE } \\
\text { METHOD }\end{array}$ & $\begin{array}{l}\text { SOLIDS } \\
\text { VoLUTE } \\
\text { METHOD }\end{array}$ & $\begin{array}{l}\text { SOLIOS } \\
\text { VOLuAE } \\
\text { UPDATE }\end{array}$ & $\begin{array}{l}\text { SOURCE } \\
\text { SEE } \\
\text { FOOTMOTE }\end{array}$ & $\begin{array}{r}\text { LAST } \\
\text { PHOTO } \\
\text { DATE }\end{array}$ & $\begin{array}{l}\text { FOR } \\
\text { THESE } \\
\text { CHAMGES }\end{array}$ \\
\hline
\end{tabular}

Footnotes:

(a) BX-111 - Following Information from cosnizant Engineer, Stabilization: Puming began October 22, 1993, and was completed April 29, 1993.

Total waste: $211.7 \mathrm{Kgal}$

Supernate: $0.7 \mathrm{kgal}$

i Drainable Interstitial: $29 \mathrm{Kgal}$

10 Pumped this Month: $3.3 \mathrm{kgal}$ (3806 gallons less 521 gallon flushes) (Pumping restarted May 25, 1994, for flushing)

Total Pumped: $110.4 \mathrm{Kgal}$

Drainable Liquid Remaining: $30 \mathrm{Kgal}$

Pumeble Liquid Remaining: $8 \mathrm{kgal}$

Studge: $68 \mathrm{kgal}$ (no change)

Sal tcake: $143 \mathrm{Kgal}$ (No change)

Photos were taken May 19. 1994

(b) BY-102 - Following information from cosnizent Engineer, Stabilization:

Puniping restarted hay 30, 1994

Total waste: $341 \mathrm{Kgal}$ (no change)

Supernate: $0 \mathrm{kgal}$ (no chenge)

Draininble Interstitial Liquid: $38.7 \mathrm{kgal}$

Pumped this Month: $2.3 \mathrm{Kgal}$ (2371 gallons less 73 gallon flushes)

Total Pumped: $125.6 \mathrm{Kgal}$

Oraineble Liquid Remining: $38.7 \mathrm{Kgal}$

Pumpable Liquid Remaining: $19.7 \mathrm{kgal}$

sludge: 0 (No change)

Sal tcake: $341 \mathrm{Kgal}$ (no change) 
TABLE E-5. IAVENTORY AND STATUS BY TAMK

SINGLE-SHELL TAMKS

May 31, 1994

TANK STATUS

\begin{tabular}{|c|c|c|c|}
\hline & $\cdot$ & & STABIL/ \\
\hline TANK & $\begin{array}{l}\text { MASTE } \\
\text { MATERIAL. }\end{array}$ & $\begin{array}{c}\text { TANK } \\
\text { INTEERITY }\end{array}$ & $\begin{array}{l}\text { ISOLATIOH } \\
\text { STATUS }\end{array}$ \\
\hline
\end{tabular}

(c) Br-109 - Following information from Cognizant Engineer, Stabilization:

Puping restarted May 31, 1994

Total weste: $423 \mathrm{Kgal}$ (no change)

supernate: $0 \mathrm{Kgal}$ (no chenge)

Draininble Interstitial Liquid: $70 \mathrm{kgal}$

Puped this Month: $0.1 \mathrm{Kgal}$

No Total Puped: $93.6 \mathrm{Kgal}$

Draineble Liquid Rewaining: $77.9 \mathrm{Kgal}$

Pumpoble Liquid Remaining: $56.9 \mathrm{Kgal}$

Sludge: 83 (no change)

Sal tcake: $340 \mathrm{Kgal}$ (no change)

Sol ids volume update: April 18, 1994

(d) T-111 - Following information from Cognizant Engineer, stabilization:

Puiping begen May 17, 1994

Total waste: 453

Supernate: $0 \mathrm{Kgal}$ (pamping shoued that the $9.0 \mathrm{Kgal}$ supernate estimate was not accurate: $3.0 \mathrm{Kgal}$ was more accurate.)

Draininble Interstitial Liquid: $55 \mathrm{kgal}$

Puped this Month: $3.1 \mathrm{Kgal}$ (4200 gallons less 1100 gallon flushes)

Total pumped: $3.1 \mathrm{kgal}$

Drainable Liquid Remaining: $55 \mathrm{Kgal}$

Pumpable Liquid Remaining: $49 \mathrm{Kgal}$

Sludge: $453 \mathrm{kgal}$

Sal tcake: $0 \mathrm{Kgal}$ (no change)

Sol ids volume update: May 31, 1994
SOLIDS VOLUnE

DRAIN- PUMP-

SUPER- ABLE PUNPED ABLE ABLE

REMAIN REMAIN

(Kgal) (Kgal)
SLLDGE SALTCAKE

(Kgal) (Kgal)

\begin{tabular}{lcccl}
\hline & & & & \\
\hline & & SOLIDS & & SEE \\
UPOATE & & FOOTMOTES \\
LIOUIDS SOLIDS & SOLIOS & SOURCE & LAST & FOR \\
VOLUNE VOLUNE VOLUME & SEE & PHOTO & THESE \\
METHOD METHOD & UPDATE FOOTMOTE & DATE & CHAMGES
\end{tabular}

VOLUEE DETERMIMATION 
WHC-EP-0182-74

APPENDIX F

PERFORMANCE SUMMARY

F-1 


\section{WHC-EP-0182-74}

This page intentionally left blank. 
TABLE F-1. PERFORMANCE SUMMARY (Sheet 1 of 2)

WASTE VOLUMES (Kgallons)

May 31, 1994

\begin{tabular}{|c|c|c|}
\hline \multicolumn{3}{|c|}{$\begin{array}{l}\text { INCREASESIDECREASES IN WASTE VOLUMES } \\
\text { STORED IN DOUBLE-SHELL TANKS }\end{array}$} \\
\hline SOURCE & $\begin{array}{l}\text { THIS } \\
\text { MONTH }\end{array}$ & $\begin{array}{l}\text { FY1994 } \\
\text { TO DATE }\end{array}$ \\
\hline 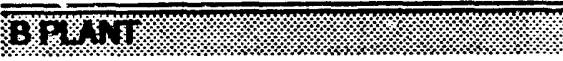 & (2) & 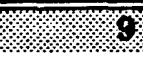 \\
\hline PUREX TOTAL (1) & 0 & 41 \\
\hline 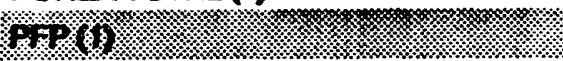 & 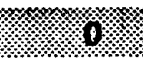 & ?. \\
\hline T PLANT (1) & 0 & 0 \\
\hline $\sin \cdot \sin (1)$ & 然. & 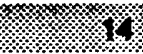 \\
\hline $300 / 400$ AREAS (1) & 0 & 12 \\
\hline 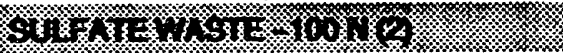 & (x) & 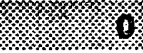 \\
\hline MINI-RUN (14) & 0 & 67 \\
\hline 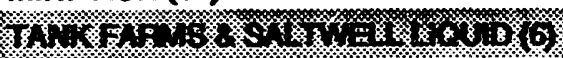 & 2. & 193 \\
\hline
\end{tabular}

$\grave{\omega}$
INCREASESJDECREASES IN WASTE VOLUMES STORED IN SINGLE-SHELL TANKS

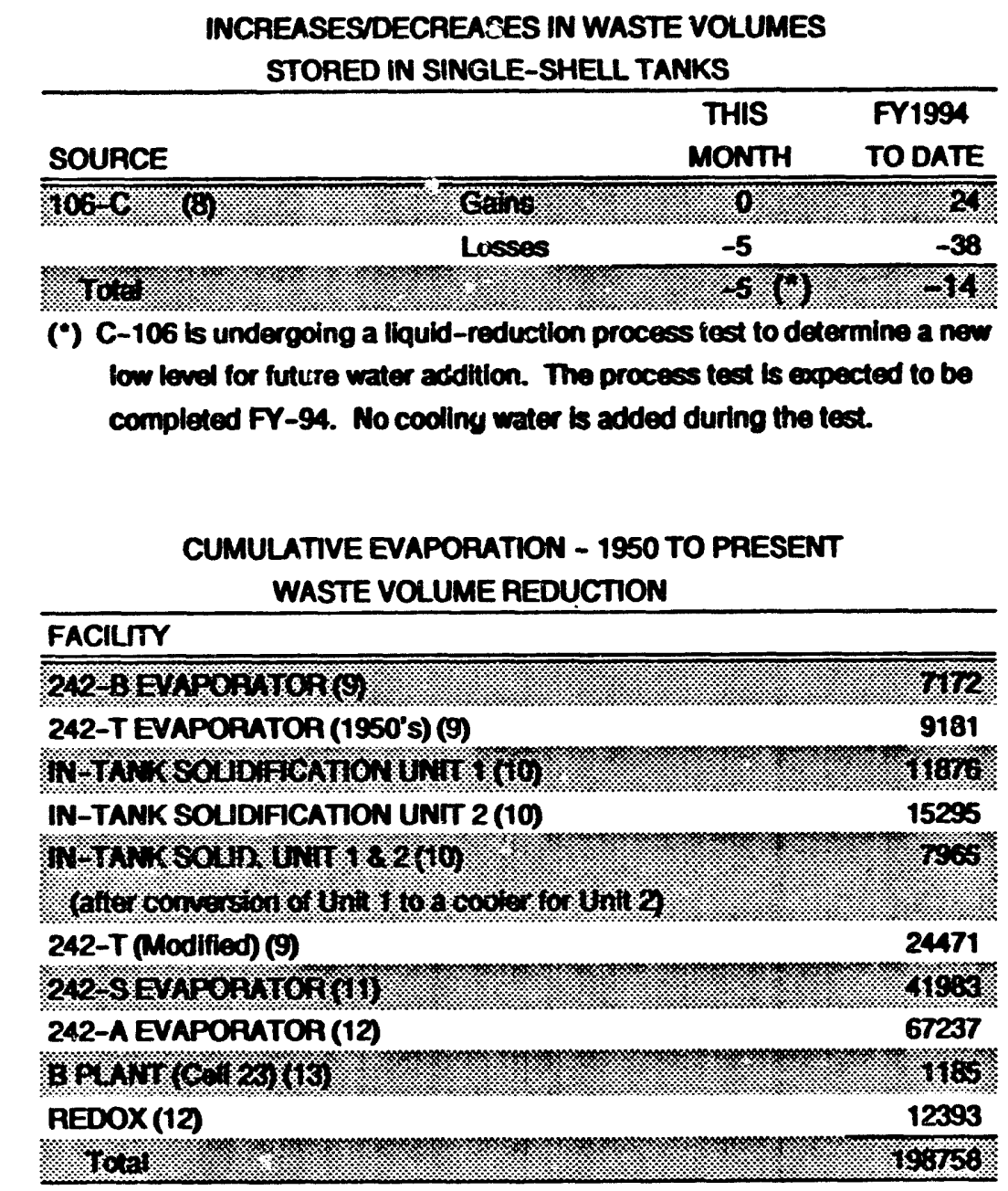

(') C-106 is undergoing a liquid-reduction process fest to determine a new low level for future water addition. The process test is expected to be completed FY-94. No cocling water is added during the test.

CUMULATIVE EVAPORATION - 1950 TO PRESENT WASTE VOLUME REDUCTION

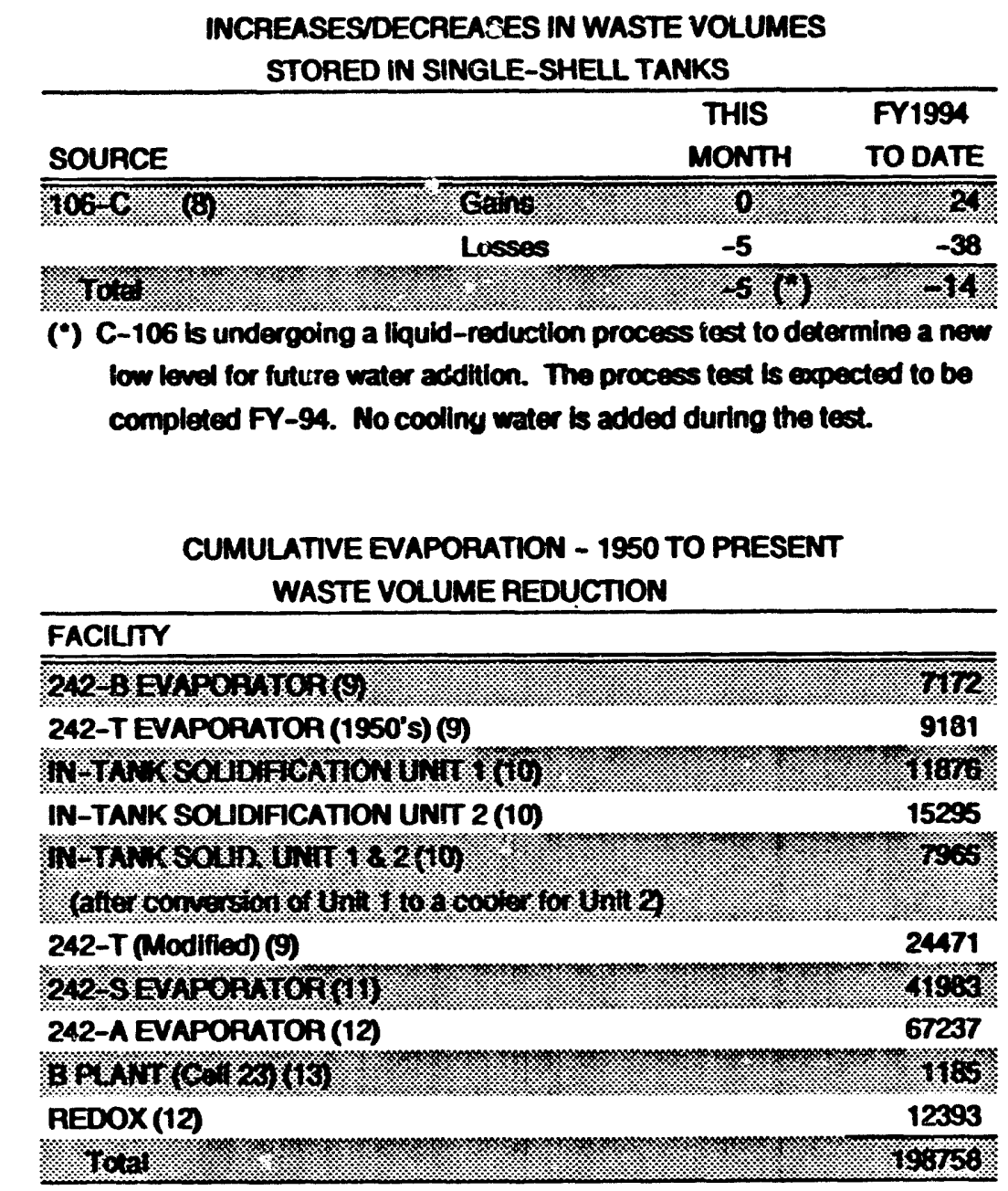

Note: 24L-A Evaporator was restarted April 15, 1994.
31

Instrument change (7)

Unknown (5)

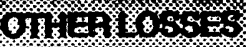

Slurry decrease (3)

Evaporation (4)

Instrument change (n)

Unknown (5)

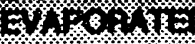

GROUTED

5

0

$+/-1$ Kgal differences are the rasult of rounding 
MHC-EP-0182-74

TABLE F-1. PERFORMANCE SUMMARY

(Sheet 2 of 2)

Footnotes:

\section{INCREASES/PECREASES IR MASTE VOLUMES}

(1) Including Flush

(2) Sulfate waste is generated from lon exchenge beckflushing and sand fllter clean out, resulting in sulfate waste $\left(\mathrm{Na}_{2} \mathrm{SO}_{4}\right)$.

(3) Slurry increase/growth is caused by gas generotion within the waste. The gas which is trapped in the waste expands in the tank causing the surface level and volume to increase. slurry dacrease results from the perlodic release of gas in the waste.

(4) Aging waste tanks

(5) Unknown waste gains or losses wa be the result of rounding calculations, clean water slowly leaking through a valve, changes in levels (expansion/contraction) because of enbient temperature chonges. different measuring devices being used by Tank farm operatore, transfers taking place during the end of the wonth, Tank Farm activities such as miscellaneous water additions not essociated with facillity waste generation, or the addition of water which is added to aging waste tanks and then evaporated off.

Includes Tank forms miscelleneous flushes (flushes ore used to "clean out" pipelines and roduce personnel exposure, reduce potential for waste incompatibility, prevent line plugging, and reduce waste content of potential spills or leaks), and saltwell liquid, which results from pumping of single-shell tanks to double-shell tanks.

(7) Liquid level measurement instrument changes from the automatic fic to manual tape (and vice verea) result in unusual gains or losses because the manual tape may rest on on uneven crust surface giving - different reading from that of the automatic FIC. These instrument changes ore made then the automatic FIC is out of service and the reading from the manual tape is used for reporting purposes. The reported reading reverts back to the automatic fic then it is repaired.

(8) Woter is periodically added 106-C to provide evaporative cooling. Losses due to evaporation are calculated assuming all losses ore evaporat ive losses. Some drywells are monitored weekly and some are monitored every two weeks on tank 106-C. If there are any indications of leak from this tank, the assumption that all losses are due to eveporation will be reevaluated.

\section{MASTE VQLUME REDUCTION}

(9) Currently inoperative. These evaporator systens (242-B and 242-T) were installed in 1952 in each of the two operating areas to renove water from the waste, and ran for approximately 4 yr after which both units were shut down. The 242-T Evaporator was reactivated in Deceaber 1965, and shut down again in April 1976.

(10) Currently inoperative. These two in-tank solidification (ITS) units provided in-tank heating to promote in-tank boilling or evaporation. The ITS Unit 1 atarted up March 1965, and ITS Unit 2 started up February 1968. In August 1971, ITS Unit 1 was converted fram an evaporator to a cooler for ITS Unit 2. Both unite were shut down June 1974.

(11) Currently inoperative. The 242-5 Evaporator-Crystallizer was started up Wovenber 1973, and shut down March 1980, when its processing campsign was completed. It is in standby mode with no future aission. This evaporator operates under vacum, caploying evaporative concentration with subsequent crystallization and precipitation of salt crystals.

(12) Currently operative. The 242-A Eveporator-Crystallizer was started up March 1977, and shut doun April 1989 because of regulotory issuse, and rawined shut down for subequent uporeding. This ovaporator operates under vacum, cuploying evaporative concentration with subsequent crystallizetion and precipitation of ealt crystals (forming saltcake). The evaporator was restarted on April 15, 1994.

(13) Currently inoperative. Additional concentration of wastes was completed by using the concentrators at REDOX and B Plant. The REDOX concentrator was used from July 1967 to June 1972, while the B Plant concentrator was used from July 1967 to February 1968.

(16) Waste generated for training and testing purposes prior to Evaporator restart. 
WHC-EP-0182-74

\section{APPENDIX G}

\section{PUMPING RECORD}

LIQUID STATUS AND PUMPABLE LIQUID

REMAINING IN TANKS 
WHC-EP-0182-74

This page intentionally left blank.

G-2 
TABLE G-1. PUMPING RECORD

May 31, 1994

(Kgallons)

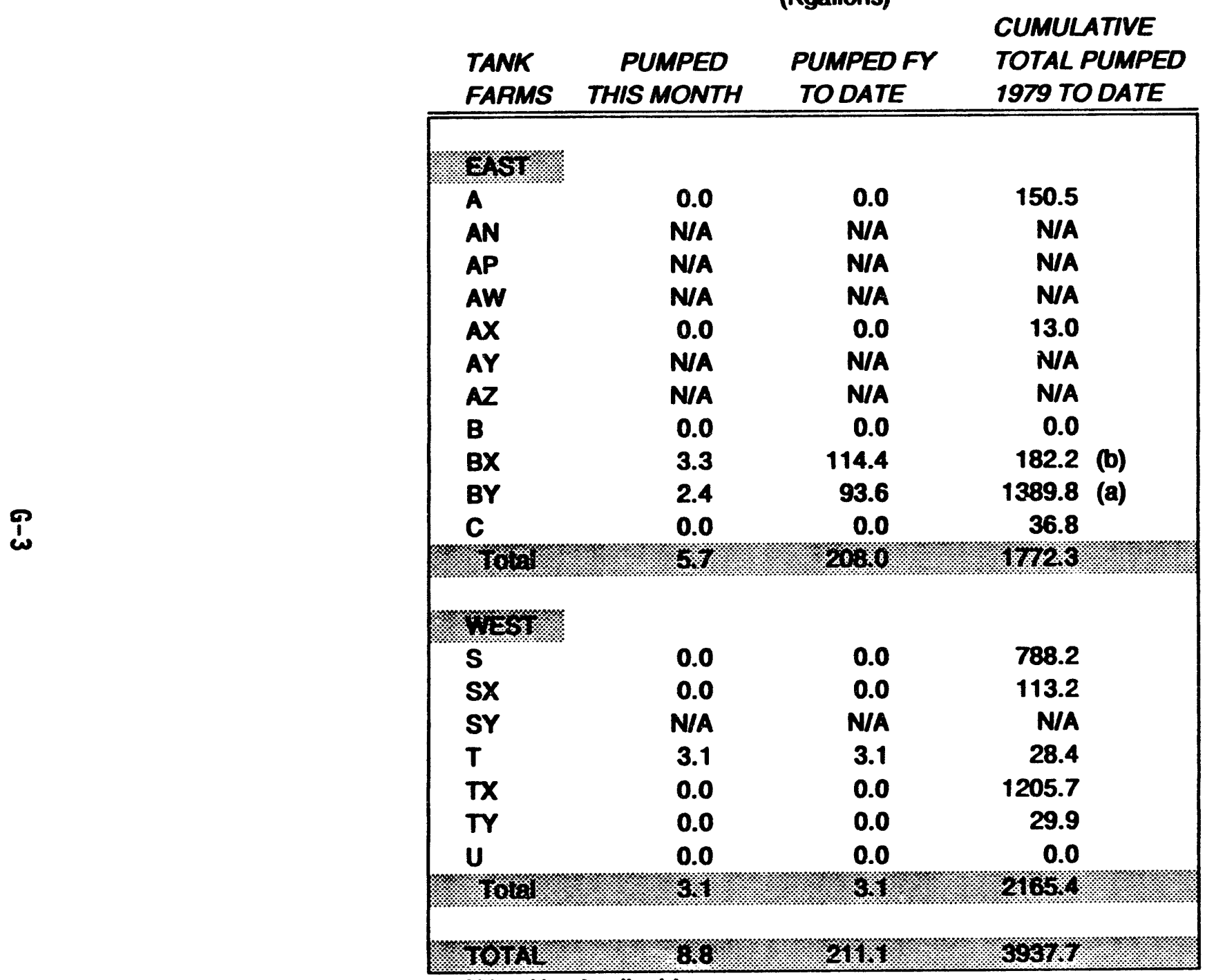

NA $=$ Not Applicable

(a) The total volume pumped was adjusted by the Single-Shell Tanks Cognizant Engineer to account for the $14 \%$ miscallbration of the constant velocity transmitter and the amount of flush water used. DIL. DLR and PLP volumes were recalculated, based on the observed porosity in 102 and $109-B Y$.

(b) Recheck of data sheets for January 1994 revealed incorrect totalizer reading was used in the calculatic.m. Recalculation corrections by Single-Shell Tanks Cognizant Engineer for January and February are included in above volumes. 


\section{TABLE G-2. LIQUID STATUS AND PUMPABLE LIQUID REMAINING IN TANKS}

May 31, 1994

Waste Volumes (Kgallons)

\begin{tabular}{|c|c|c|c|c|}
\hline $\begin{array}{l}\text { TANK } \\
\text { FARMS } \\
\end{array}$ & $\begin{array}{l}\text { SUPERNATANT } \\
\text { LIQUID } \\
\end{array}$ & $\begin{array}{l}\text { DRAINABLE } \\
\text { INTERSTITIAL } \\
\text { LIQUID } \\
\end{array}$ & $\begin{array}{c}\text { DRAINABLE } \\
\text { LIQUID } \\
\text { REMAINING }\end{array}$ & $\begin{array}{l}\text { PUMPABLE } \\
\text { LIQUID } \\
\text { REMAINING } \\
\end{array}$ \\
\hline \multicolumn{5}{|l|}{ EAST } \\
\hline A & 9 & 441 & 450 & 390 \\
\hline AN & 4647 & 37 & 4684 & N/A \\
\hline AP & 6393 & 0 & 6393 & N/A \\
\hline AW & 3785 & 159 & 3944 & N/A \\
\hline$A x$ & 3 & 370 & 373 & 304 \\
\hline AY & 1481 & 2 & 1483 & N/A \\
\hline$A Z$ & 1804 & 4 & 1808 & N/A \\
\hline B & 15 & 164 & 179 & 80 \\
\hline$B X$ & 31 & 134 & 167 & 93 \\
\hline BY & 0 & 778 & 778 & 596 \\
\hline C & 169 & 143 & 312 & 254 \\
\hline Woit & 1645\% & 22232 & $2057 \%$ & 9712 \\
\hline \multicolumn{5}{|l|}{ WEST } \\
\hline $\mathbf{s}$ & 58 & 1376 & 1434 & 1201 \\
\hline$s x$ & 63 & 1260 & 1323 & 1178 \\
\hline SY & 876 & 237 & 1113 & N/A \\
\hline $\mathbf{T}$ & 43 & 206 & 249 & 182 \\
\hline$T X$ & 5 & 250 & 255 & 0 \\
\hline$T Y$ & 3 & 31 & 34 & 0 \\
\hline $\mathbf{u}$ & 168 & 1138 & 1306 & 1104 \\
\hline Oxis & 20 & 1798 & 5714 & 3660 \\
\hline OEW & 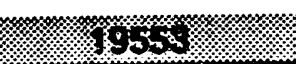 & 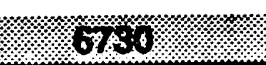 & 26285 & 5esto2 $/(11$ \\
\hline
\end{tabular}

(1) Volume based on $12.5 \%$ (sludge waste) and $45 \%$ (saltcake waste) liquid in solid (porosity) value. This is a conservative (high) estimate.

Note: $+1-1 \mathrm{Kgal}$ differences are the result of computer rounding N/A = Not applicable 
WHC-EP-0182-74

\section{APPENDIX H}

CATCH TANKS AND SPECIAL

SURVEILLANCE FACILITIES

$H-1$ 


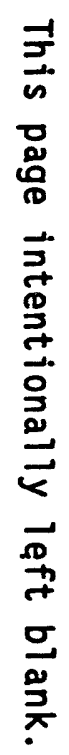




\section{TABLE H-1. EAST AND WEST AREA CATCH TANKS AND SPECIAL SURVEILLANCE FACILITIES \\ ACTIVE - still running transfers through the associated diversion boxes or pipeline encasements}

May 31, 1994

\section{VOLUME}

OF

CONTENTS MONITORED

FACILITY

LOCATION PURPOSE (receives waste from:)

(Gallons)

BY

REMARKS

\section{EAST AREA}

\section{1-A-302-A}

241-ER-311

$241-A X-152$

$241-A Z-151$

$241-A Z-154$

244-BX-TK/SMP

244-A-TKVSMP

AR-204

A-417

Vent Station Catch Tank

A FARM
B PLANT
AX FARM
AZ FARM
AZ FARM
BX COMPLEX
A COMPLEX
AY FARM
A FARM

A-151 DB

ER-151, ER-152 DB

AX-152 DB

AZ-152 DB, AZ LOOP SEAL

AZ-102 HTG COIL STEAM CONDENSATE

DCRT - RECEIVES FROM SEVERAL FARMS

DCRT - RECEIVES FROM SEVERAL FARMS

RR CARS DURING TRANSFER TO REC. TKS

A-702 PROCESS CONDENSATE

CROSS COUNTRY TRANSFER LINE

$\stackrel{T}{1}$

\begin{tabular}{|c|c|}
\hline 0 & \\
\hline $241-T X-302-C$ & TX FARM \\
\hline $241-U-301-B$ & U FARM \\
\hline $1-U X-302-A$ & U PLANT \\
\hline $241-S-304$ & S FARM \\
\hline 244-S-TK/SMP & S FARM \\
\hline 44-TX-TK/SMP & TXFAF \\
\hline
\end{tabular}

TX-154 DB

$U-151, U-152, U-153, U-252 D B$

UX-154 DB

S-151 DB

DCRT - RECEIVES FROM SEVERAL FARMS

DCRT - RECEIVES FROM SEVERAL FARMS

$\begin{array}{rll}1724 & \text { CASS/FIC } & \text { PUMPED 08/11/92 } \\ 8504 & \text { CASS/FIC } & \text { PUMPED 05/29/91 } \\ 1751 & \text { MANUALIY } & \text { DIAL O/S, USING ZIP CORD, PUMPED 08/29/9 } \\ 4050 & \text { CASS/FIC } & \text { VOLUME CHANGES DAILY } \\ 0 & \text { CASSMT } & \text { AUTOMATIC PUMP } \\ 20551 & \text { MANUALIY } & \text { USING MANUAL TAPE FOR TANK } \\ 7135 & \text { MCS } & \\ 360 & \text { DIP TUBE } & \text { ALARMS ON CASS } \\ 34220 & \text { DIP TUBE } & \\ 649 & \text { MT } & \\ & & \\ 3952 & \text { CASS/FIC } & \text { FIC REPAIRED } \\ 7007 & \text { CASS/FIC } & \text { RETURNED TO SERVICE 12/30/93 } \\ 12131 & \text { CASS/MFIC } & \\ 3844 & \text { RS } & \text { OPERATIONAL 10/91, REPLACED S-302-A } \\ 11087 & \text { MANUALLY } & \text { CWF } \\ 5887 & \text { MANUALIY } & \text { MT }\end{array}$

Total active facilities 16

\begin{tabular}{|c|c|}
\hline LEGEND. & 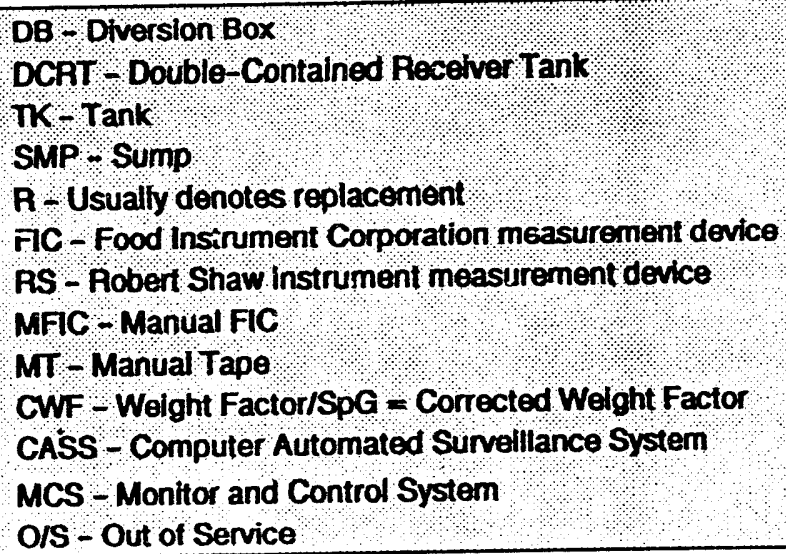 \\
\hline
\end{tabular}




\section{TABLE H-2. EAST AREA CATCH TANKS AND SPECIAL SURVEILLANCE FACILITIES}

INACTIVE - no longer receiving waste transfers

May 31, 1994

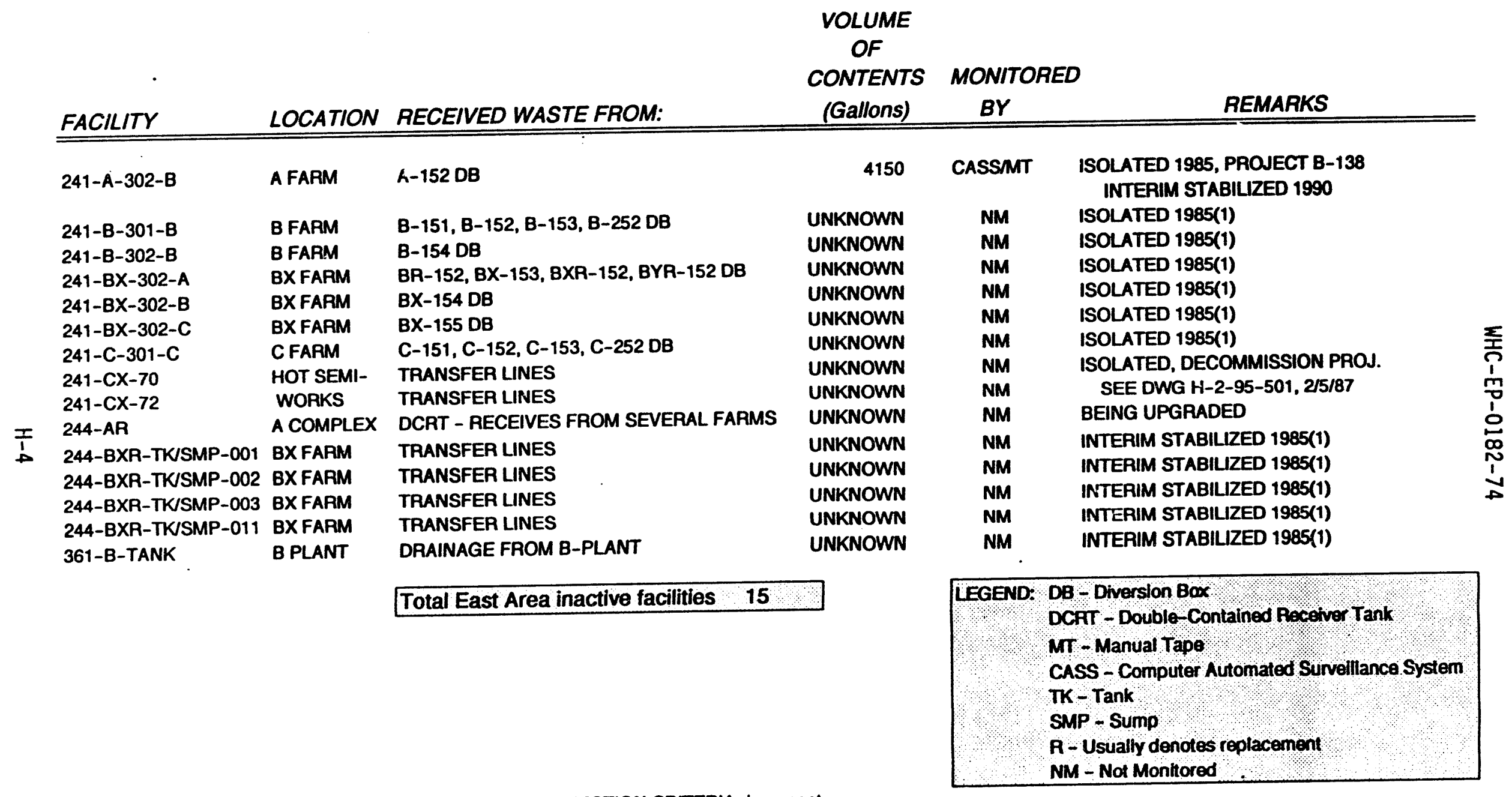

(1) SOURCE: WASTE STORAGE TANK STATUS \& LEAK DETECTION CAITERIA document 
TABLE H-3. WEST AREA CATCH TANKS AND SPECIAL SURVEILLANCE FACILITIES

INACTIVE - no longer receiving waste transfers

May 31, 1994

VOLUME

OF

CONTENTS

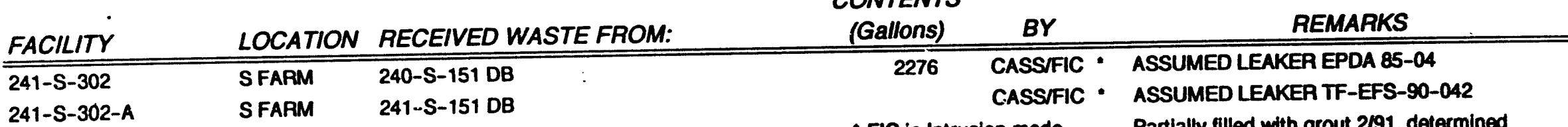

- FIC in Intrusion mode Partially filled with grout 2191, determined

still ass. med leaker after leak test

\begin{tabular}{|c|c|c|c|}
\hline $241-S-302-B$ & SFARM & S ENCASEMENTS & UNKNOWN \\
\hline $241-S X-304(302)$ & SXFARM & SX-152 TRANSFER BOX, SX-151 DB & UNKNOWN \\
\hline $241-T X-302$ & TXFAFM & TX-153 DB & UNKNOWN \\
\hline $241-T X-302-X-B$ & TXFARM & TXENCASEMENTS & UNKNOWN \\
\hline 241-TX-302-B & TXFARM & TX-155 DB & 1460 \\
\hline $241-T Y-302-A$ & TY FARM & $T X-153 D B$ & UNKNOWN \\
\hline $241-T Y-302-B$ & TY FARM & TY ENCASEMENTS & UNKNOWN \\
\hline 244-U-TKUSMP & U FARM & DCRT - RECEIVES FROM SEVERAL FARMS & UNKNOWN \\
\hline 244-UR VAULT & U FARM & TRANSFER LINES & UNKNOWN \\
\hline 244-UR-TKJSMP-001 & U FARM & TRANSFER LINES & UNKNOWN \\
\hline 244-UR-TKISMP-002 & U FARM & TRANSFER LINES & UNKNOWN \\
\hline 244-UR-TK/SMP-003 & U FARM & TRANSFER LINES & UNKNOWN \\
\hline 244-TXR VAULT & TXFARM & TRANSFER LINES & UNKNOWN \\
\hline 244-TXR-TKUSMP-001 & TXFARM & TRANSFER LINES & UNKNOWN \\
\hline 244-TXR-TKUSMP-002 & TXFARM & TRANSFER LINES & UNKNOWN \\
\hline 244-TXR-TK/SMP-003 & TXFARM & TRANSFER LINES & UNKNOWN \\
\hline 361-T-TANK & TPLANT & $\begin{array}{l}\text { DRAINAGE FROM T-PLANT } \\
\text { TAINAGF FROM U-PLANT }\end{array}$ & $\begin{array}{l}\text { UNKNOWN } \\
\text { UNKNOWN }\end{array}$ \\
\hline
\end{tabular}
ISOLATED 1985(1)

NM ISOLATED 1985(1)

$\begin{array}{lll}\text { UNKNOWN } & \text { NM } & \text { ISOLATED 1985(1) } \\ \text { UNKNOWN } & \text { NM } & \text { ISOLATED 1985(1) }\end{array}$

UNKNOWN NM ISOLATED 1985(1)

CASSMT NEW MT INSTALLED 7/16/93

NEW MT INSTALL

NM ISOLATED 1985(1)

NM NOT YET IN USE

INTERIM STABILIZED, MT REMOVED 1985(1) INTERIM STABILIZED, MT REMOVED 1985(1) INTERIM STABILIZED, MT REMOVED 1985(1) INTERIM STABILIZED, MT REMOVED 1985(1) INTERIM STABILIZED, MT REMOVED 1984(1) INTERIM STABILIZED, MT REMOVED 1984(1) INTERIM STABILIZED, MT REMOVED 1984(1) INTERIM STABILIZED, MT REMOVED 1984(1) ISOLATED 1985(1)

361-U-TANK U PLANT

DRAINAGE FROM U-PLANT

UNKNOWN

\begin{tabular}{|l|}
\hline LEGEND: DB - Diversion Box \\
DCRT - Double-Contained Recelver Tank \\
TK - Tank \\
SMP - Sump \\
R - Usually denotes replacement \\
FIC - Food Instrument Corporation \\
MT - Manual Tape \\
O/S - Out of Service \\
CASS - Computer Automated Surveillance System \\
NM - Not Monitored \\
\hline
\end{tabular}


WHC-EP-0182-74

\section{APPENDIX I \\ LEAK VOLUME ESTIMATES}




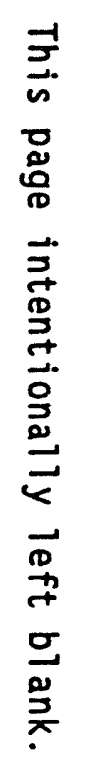

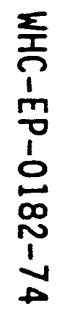


WHC-EP-0182-74

TABLE I-1. SINGLE-SHELL TANK LEAK VOLUME ESTIMATES (4) (Sheet 1 of 6) Date Declared Associated Interim

Confirmed or Volume (2)(4) KiloCuries

Stabilized

Tank No. Assumed Leaker (3) (Gallons) $137 \operatorname{cs}(10)$ Date (12) Updated Reference

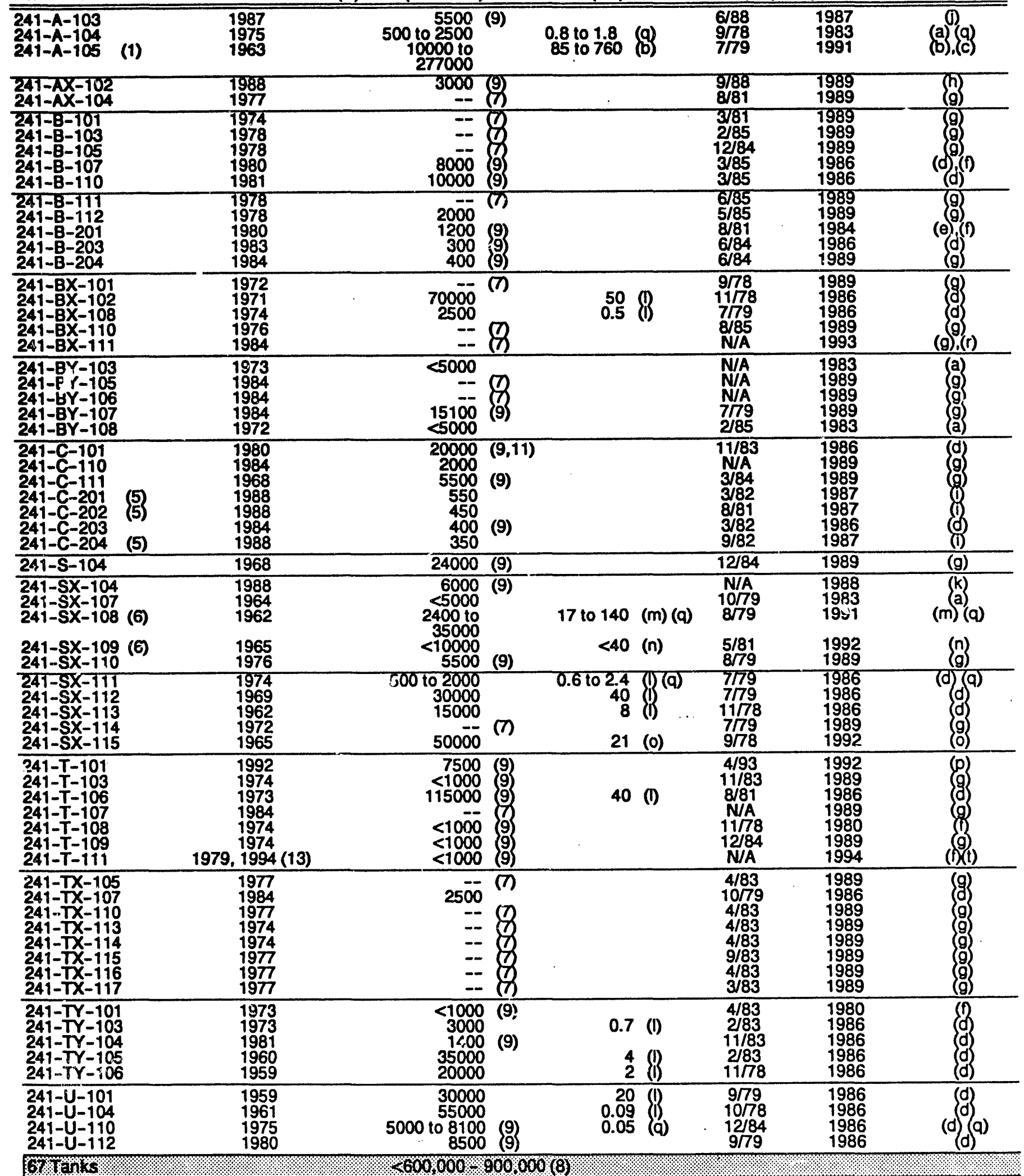
N/A = .10t applicable (not yet interim stabilized) FOOTNOTES: SEE NEXT PAGE 


\section{Footnotes:}

Current estimates (see reference b) are that $610 \mathrm{kgal}$ of cooling water was added to Tank 241-A-105 from Noveaber 1970 to December 1978 to aid in evaporative cooling. In accordance with Dangerous Waste Regulations (Washington Adninistrative Code 173-303-070 (2)(a)(ii), as amended, Washington State Department of Ecology, 1990, Olymia, Washington), any of this cooling water that has been added and subsequently leaked from the tank must be classified as a waste and should be included in the total leak volume. In August 1991, the leak volume estimate for this tank was updated in accordance with the MAC regulations. Previous estimates excluded the cooling water leaks from the total leak volume estimates because the waste content (concentration) in the cooling water which leaked should be much less than the original liquid waste in the tank (the sludge is relatively insoluble). The total leak volume estimate in this report (10 Kgal to $277 \mathrm{Kgal}$ ) is based on the following (see References).

1. Reference (b) contains an estimate of $5 \mathrm{kgal}$ to $15 \mathrm{Kgal}$ for the initial leak prior to August 1968.

2. Reference (b) contains an estimate of $5 \mathrm{Kgal}$ to $30 \mathrm{Kgal}$ for the leak while the tank was being sluiced from August 1968 to November 1970.

3. Reference (b) contains an estimate of $610 \mathrm{kgal}$ of cooling water added to the tank from Noverber 1970 to December 1978 but it was estimated that the leakage was small during this period. This reference contains the statement "Sufficient heat was generated in the tank to evaporate most, and perhaps nearly all, of this water." This results in a low estimate of zero gallons leakage from November 1970 to December 1978.

4. Reference (c) contains an estimate that 373 to $410 \mathrm{Kgal}$ evaporated out of the tank from November 1970 to December 1978. Subtracting the minimum evaporation estimate from the cool ing water added estimate provides a range from 0 to $232 \mathrm{Kgal}$ of cooling water leakage from November 1970 to December 1978.

Low Estimate

Prior to August 1968

August 1968 to November 1970

Novenber 1970 to December 1978

Totals

$$
\begin{array}{r}
5,000 \\
5,000 \\
10,000
\end{array}
$$

High Estimate

$$
\begin{array}{r}
15,000 \\
30,000 \\
232,000 \\
\hline 277,000
\end{array}
$$

These leak volume estimates do not include (with some exceptions), such things as: (a) cooling/raw water leaks, (b) intrusions (rain infiltration) and subsequent leaks, (c) leaks inside the tank farm but not through the tank liner (surface leaks, pipeline leaks, leaks at the joint for the overflow or fill lines, etc.), and (d) leaks from catch tanks, diversion boxes, encasements, etc.

In many cases, a leak was suspected long before it was identified or confirmed. For example, reference (d) shows that Tank 241-U-104 was suspected of leaking in 1956. The leak was "confirmed" in 1961. This report lists the "assumed leaker" date as 1961. Using present standards, Tank 241-U104 would have been declared as assumed leaker in 1956. In 1984, the criteria designations of "suspected leaker," "questionable integrity, "confirmed leaker," "declared leaker," "borderl ine," and "dormant," were merged into one category now reported as "assumed leaker." See reference (f) for explanation of when, how long, and how fast some of the tanks leaked. It is highly likely that there have been undetected leaks from single-shell tanks because of the nature of their design and instrumentation.

There has been an effort in the past two years to reevaluate these leak volume estimates. During the Fy 1993 funding reviews, this reevaluation of leak volumes was given a priority which resulted in this activity no longer being funded. The priority versus funding will be reevaluated as part of the prior to FY 1994 budget planning.

The leak volume estimate date for these tanks is before the "declared leaker" date because the tank was in a "suspected leaker" or "questionable integrity" status; however, a leak volume had been estimated prior to the tank being reclassified.

(6) The increasing radiation levels in drywells and laterals associated with these three tanks could be indicative of a continuing leak or movement of existing radionuclides in the soil. There is no conclusive way to confirm these observations.

(7) Methods were used to estimate the leak volumes from these 19 tanks based on the assumption that their cumulative leakage is approximately the same as for 18 of the 24 tanks identified in footnote (10). For more details see reference $(g)$. The total leak volume estimate for these tanks is 150 $\mathrm{Kgal}$ (rounded to the nearest $10 \mathrm{Kgal}$ ), for an average of approximately $8 \mathrm{Kgal}$ for each of the 19 tanks. 


\section{WHC-EP-0182-74}

\section{TABLE 1-1. SINGLE-SHELL TANK LEAK VOLUME ESTIMATES (Sheet 3 of 4 )}

(8) The total has been rounded to the nearest $50 \mathrm{kgal}$. Upperbound values were used in many cases in developing these estimates. It is likely that some of these tanks have not actually leaked.

(9) Leak volume estimate is based solely on observed liquid level decreases in these tanks. This is considered to be the most accurate method for estimating leak volumes.

(10) The curie content listed is as listed in the reference document and is not decayed to a consistent date; therefore, a cumulative total is inappropriate.

(11) Tank 241-C-101 experienced a liquid level decrease in the late 1960s and was taken out of service and pumped to a "minimum heel" in Decenber 1969. In 1970, the tank was classified as a

"questionable integrity" tank. Liquid level data show decreases in level throughout the 1970s and the tank was saltwell pumped during the 1970s, ending in April 1979. The tank was reclassified as a "conf irmed leaker" in January 1980. See reference (q) and (s): refer to reference (s) for information on the potential for there to have been leaks from other c-farm tanks (specifically, c102, C-103, and (-109).

(12) These dates indicate when the tanks were declared to be interim stabilized. In some cases, the official interim stabilization documents were issued at a later date. Also, in some casis, the field work associated with interim stabilization was completed at an earlier date.

(13) An unexplained 0.30 inch level decrease was observed in 1974 and the tank was then declared "Questionable Integrity" and removed from service. Tank T-111 was declared an assumed re-leaker on February 28, 1994, due to a decreasing trend in surface level measurement. 
WHC-EP-0182-74

TABLE 1-1. SINGLE-SHELL TANK LEAK VOLUME ESTIMATES

References:

(Sheet 4 of 4 )

(a) Murthy, K.S., et al, June 1983, Assessment of Single-Shell Tank Residual Liquid Issues at Hanford Site, Mashington, PNL-4688, Pacific Northwest Laboratory, Richland, Washington.

(b) WHC, 1991a, Iank 241-A-105 Leak Assessment, WHC-MR-0264, Westinghouse Hanford Company, Richland, Washington.

(c) WHC, 1991b, Iank 241-A-105 Evaporation Estimate 1970 Through 1978, WHC-EP-0410, Westinghouse Hanford Company, Richtand, Washington.

(d) Smith, D. A., January 1986, Single-Shell Tank I solation Safety Analysis Report, SD-WM-SAR-006, Rev. 1. Westinghouse Kanford Company, Richland, Washington.

(e) McCann, D. C., and T. S. Vail, September 1984, Haste Status Summary, RHO-RE-SR-14, Rockwell Hanford Operations, Richland, Washington.

(f) Catlin, R. J., March 1980, Assessment of the Surveill ance Program of the High-Level Waste Storage Tanks at Hanford, Hanford Engineering Development Laboratory, Richland, Washington.

(g) Baumhardt, R. J., May 15, 1989, Letter to R. E. Gerton, U.S. Department of Energy-Richland Operations Office, Single-Shell Tank Leak Volumes, Westinghouse Hanford Company, Richland, Washington.

(h) WHC, 1990a, Occurrence Report, Surface Level Measurement Decrease in Single-Shell Tank 241-AX-102, WHC-U0-89-023-TF-05, West inghouse Hanford Company, Richland, Washington.

(i) Groth, D. R. July 1, 1987, Internal Memorandum to R. J. Baumhardt, Liquid Level Losses in Tanks 241-c-201,-202 and -204, 65950-87-517, Hestinghouse Hanford Company, Richland, Washington.

(j) Groth, D. R. and G. C. Owens, May 15, 1987, Internal Memorandum to J. H. Roecker, Iank 103-A Integrity Evaluation, Westinghouse Hanford Company, Richland, Washington.

(k) Cempbell, G. D., July 8, 1988, Internal Memorandum to R. K. Helty, Engineering Investigation: Interstitial Liquid Level Decrease in Tank 241-5x-104, $13331-88-416$, Westinghouse Hanford Company, Ríchland, Washington.

(1) ERDA, 1975, Final Environmental Statement Waste Management Operations, Hanford Reservation, Richland, Washington, ERDA-1538, 2 vols., U.S. Energy Research and Development Administration, Washington, D.C.

(m) WHC, 1992a, Iank 241-SX-108 Leak Assessment, WHC-MR-0300, Westinghouse Hanford Company, Richland, Hashington.

(n) WHC, 1992b, Tank 241-5X-109 Leak Assessment, WHC-MR-0301, Westinghouse Hanford Company, Richland, Washington.

(0) WHC, 1992c, Iank 241-5X-115 Leak Assessment, WHC-MR-0302, Westinghouse Hanford Company, Richland, Washington.

(p) HHC, 1992d, Occurrence Report, "Apparent Decrease in Liquid Level in single Shell Underground Storage Tank 241-T-101, Leak Suspected; Investigation Continuing," RL-WHC-TANKFARM-1992-0073. Westinghouse Hanford Company, Richland, Washington.

(q) WHC-1990b, A History of the 200 Area Tank Farms, WHC-MR-0132, Westinghouse Hanford Company, Richland, Washington.

(r) WHC, 1993, Occurrence Report, Single-Shell Underground Haste Storage Tank 241-BX-111 Surface Level Decrease and Change From Steady State Condition, RL-WHC-TANKFARM-1993-0035, Westinghouse Hanford Company, Richland, Washington.

(s) WHC, 1993a, Assessment of Unsaturated Zone Radionuclide Contamination Around Single-Shell Tanks 241 C-105 and 241-C-106, WHC-SD-EN-TI-185, REV OA, West inghouse Hanford Company, Richland, Washington.

(t) WHC, 1994, Occurrence Report, "Apparent Liquid Level Decrease in Single Shell Underground Storage Tank 249-T-111; Declared an Assumed Re-Leaker," RL-WHC-TANKFARM-1994-0009, Westinghouse Hanford Company, Richland, Washington. 
WHC-EP-0182-74

\section{APPENDIX J}

\section{INTERIM STABILIZATION STATUS}




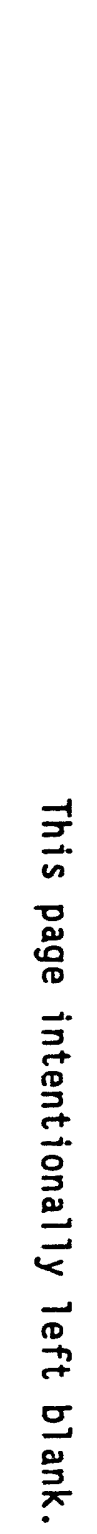

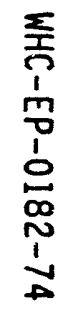


WHC-EP-0182-74

TABLE J-1. SINGLE-SHELL TANKS INTERIM STABILIZATION STATUS (Sheet 1 of 2) May 31, 1994

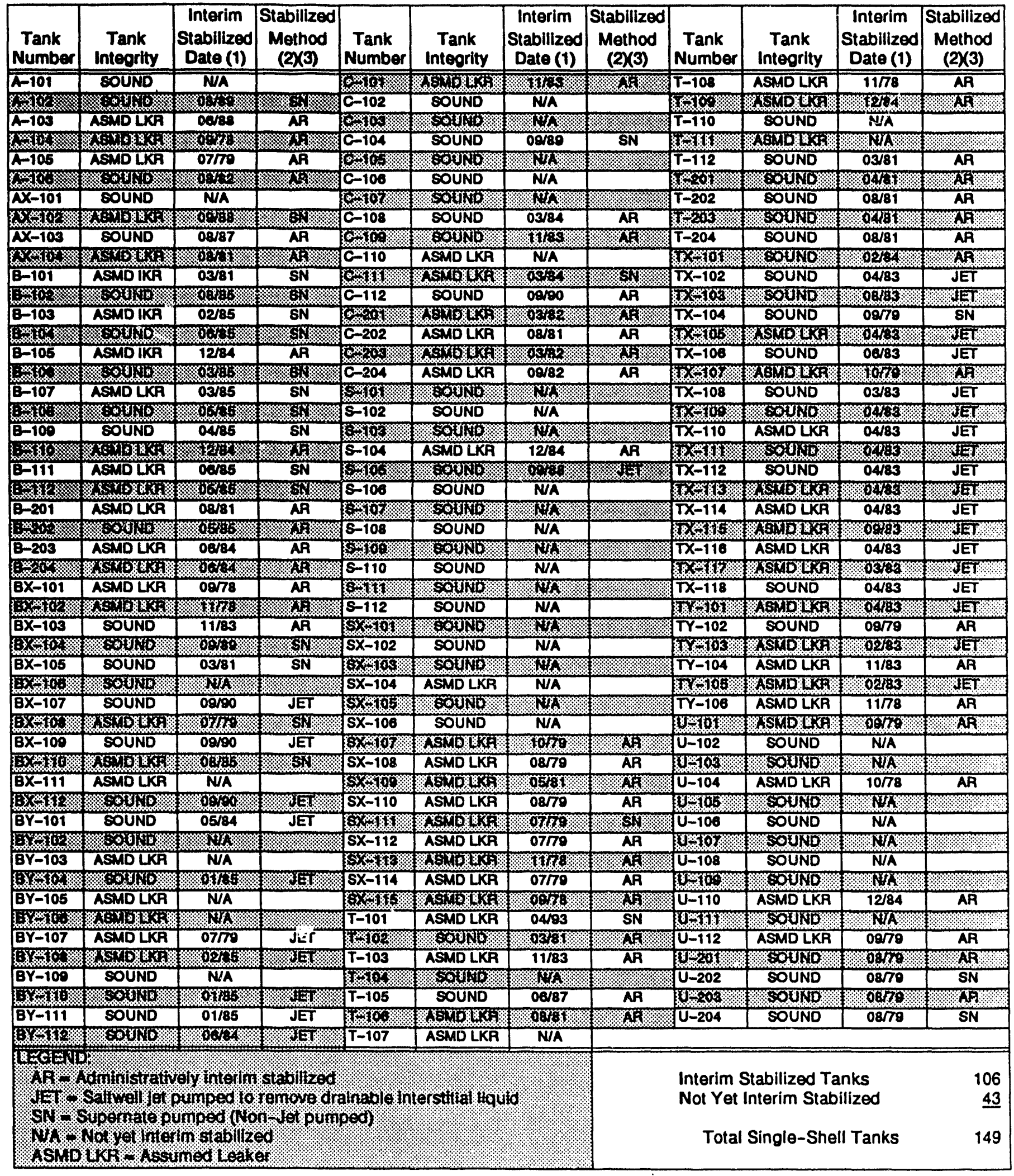

Footnotes: See next page 
WHC-EP-0182-74

TABLE J-1. SINGLE-SHELL TANKS INTERIM STABILIZATION STATUS (sheet 2 of 2)

\section{Footnotes:}

(1)

These dates indicate when the tanks were actually interim stabilized. In some cases, the official interim stabilization documents were issued at a later date.

(2) The following six tanks do not meet current established supernatant and interstitial liquid interim atabilization criteria, but did meet the criteria in existence when they were declared interim stabilized:

$$
\begin{aligned}
& \text { B-104, 110, } 111 \\
& T-102,112 \\
& U-110
\end{aligned}
$$

Interim Stabilization data are missing on four tanks. These tanks were Administratively Interim stabilized.

B-201, T-102, 112, 201 


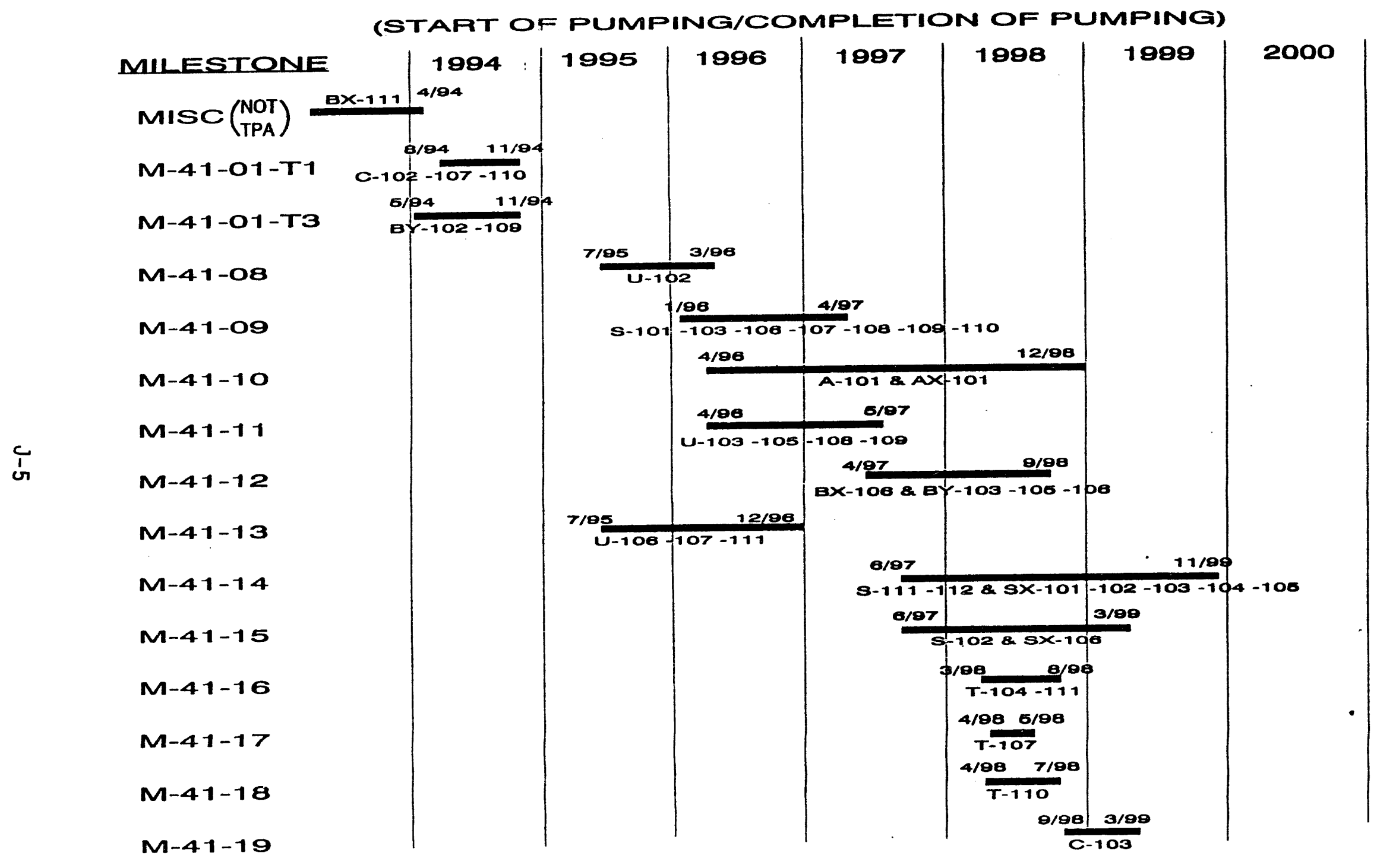

NOTE: C-106 \& C-10B NOT INCLUDED IN THIS SCHEDULE

Figure J-1. Tri-Party Agreement Single-Shell Tank Stabilization Schedule 


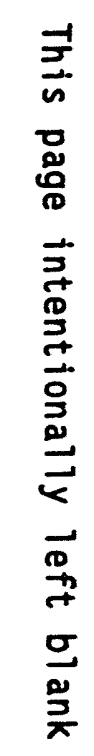


WHC-EP-0182-74

APPENDIX K

TANK FARM OPERATIONS SAMPLING SCHEDULE STATUS 
WHC-EP-0182-74

This page intentionally left blank 
TABLE K-1. TANK FARM OPERATIONS SAMPLING SCHEDULE STATUS (Sheet 1 of 3 )

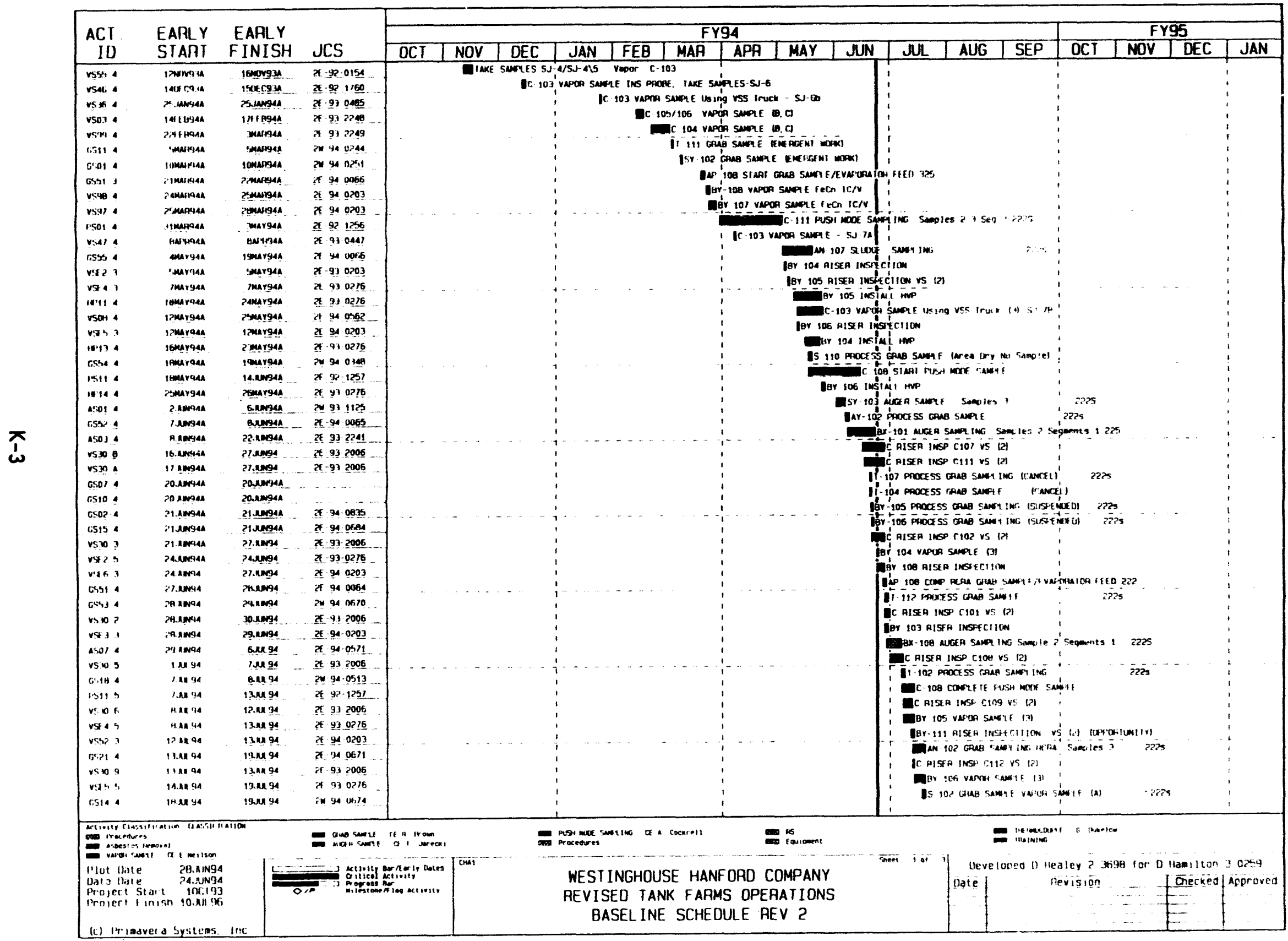


TABLE K-1. TANK FARM OPERATIONS SAMPLING SCHEDULE STATUS (Sheet 2.of 3)

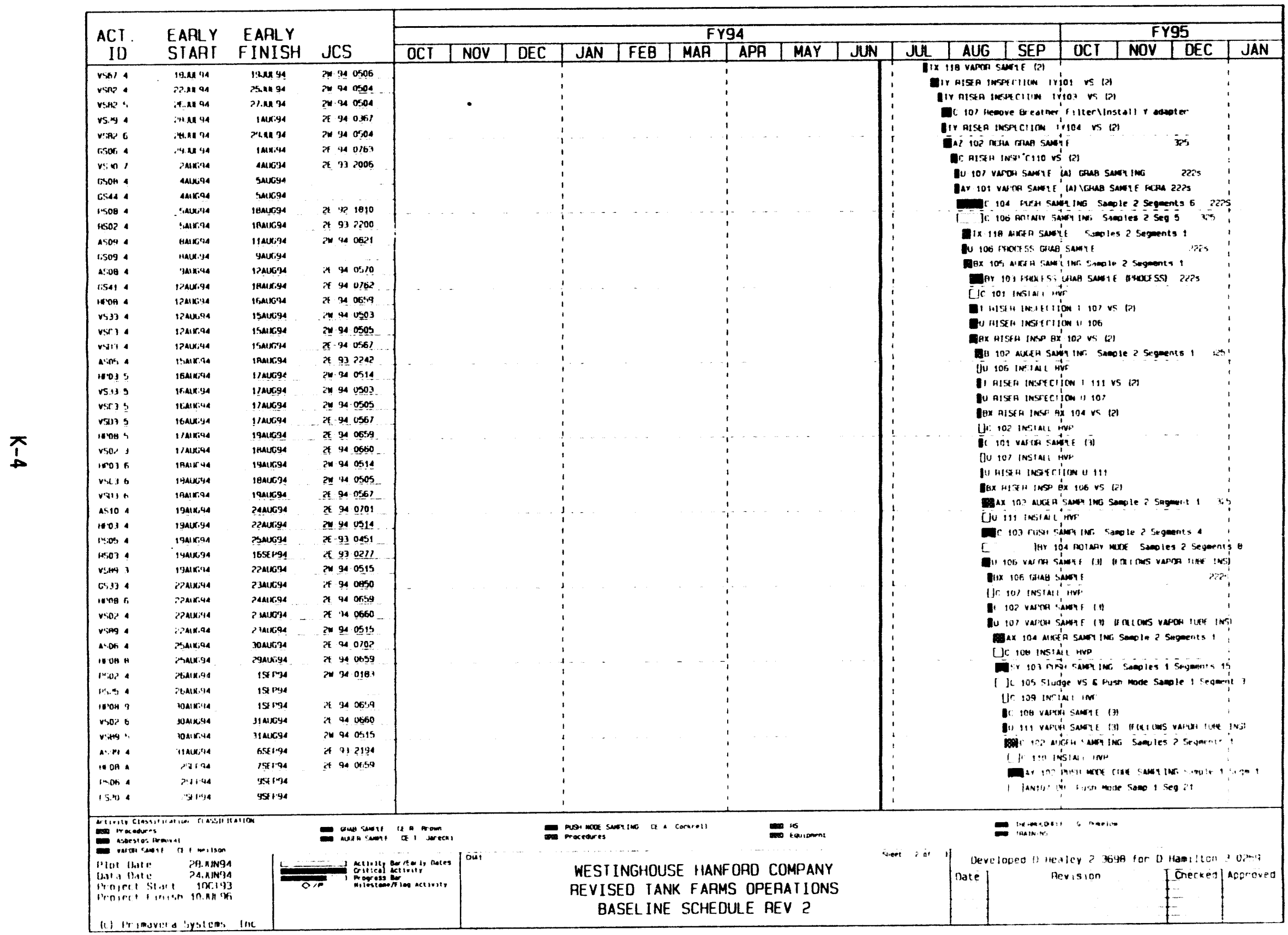


TABLE K-1. TANK FARM OPERATIONS SAMPLING SCHEDULE STATUS (Sheet 3 of 3)

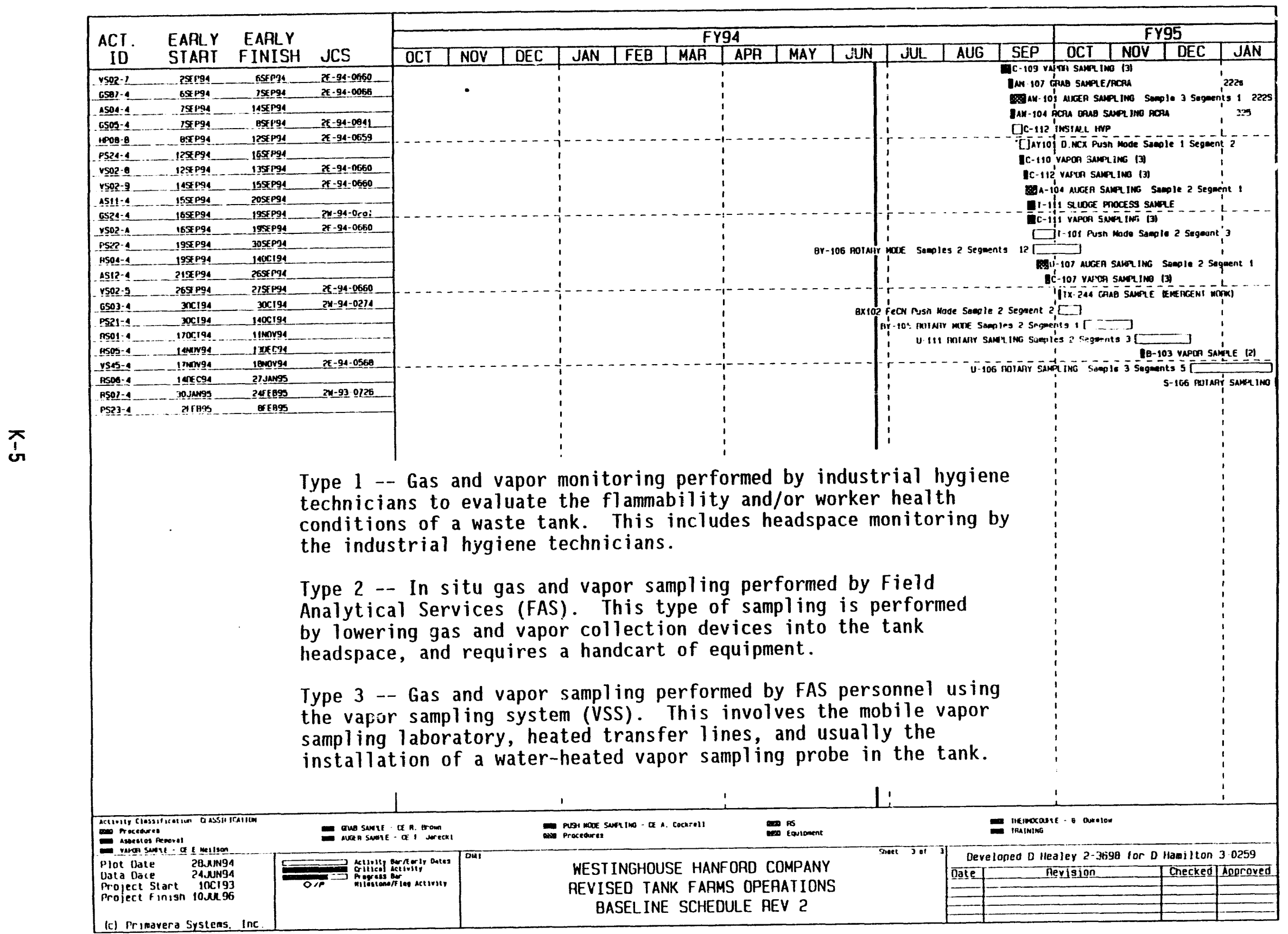


WHC-EP-0182-74

This page intentionally left blank 


\section{DISTRIBUTION}

Number of copies

OFFSITE - USA

Congress of the United States

House of Representatives

1111 Longworth Building

Washington, DC 20515-3703

Ron Wyden, Member of Congress, 3rd District

Josh Kardon, Legislative Director

House of Representatives

1431 Longworth House Office Building

Washington D. C. 20515

Jay Inslee, Member of Congress, 4th District

Atten: Louis J. Alex, Legislative Assistant

\section{U. S. Department of Energy-Headquarters}

1000 Independence Avenue, SW

Washington, D. C. 20585

Jim Antizzo

Emile Bernard

H. Calley

Teresa Fryberger

Sherry Gibson
A. Griffith
L. Gunn
D. Gupta

Kenneth Lang

J. C. Lehr

G. Mellinger

C. O'Dell

D. Pepson

J. C. Tseng

S. Woodbury

19901 Germantown Rd, Germantown, MD 20585
R. Lasky
J. Psaras
P. Worthington
$\mathrm{EH}-32.1$
NS-20 GTN
NS-20 GTN

TREV II /364

TREV II

TREV II

TREV II

TREV II

TREV II/34I

TREV II

TREV II

TREV II

TREV II $/ 160$

TREV II

TREV II

TREV II

TREV II $/ 364$

FORS / 3G-092

U. S. Department of Energy - Oak Ridge Operations Office P. 0. Box 2001

Oak Ridge, TN 37831

W. D. Adams

EW-40 
WHC-EP-0182-74

Distribution - continued

U. S. Department of Energy - Sayannah River Site P. 0. Box A

Aiken, SC 29808

$\begin{array}{ll}\text { C. Anderson } & 707-\mathrm{H} \\ \text { Michael Chandler } & 703-\mathrm{H} \\ \text { Mazen Shurrab } & 704-\mathrm{H}\end{array}$

T. C. Temple

L. Sjostrom

W. R. West

V. Wheeler.

704-8H

704-S

1

U. S. Environmental Protection Agency

Region 10

712 Swift Boulevard, Suite 5

Richl and, WA 99352

D. R. Sherwood

8

Washington State Department of Ecology

Nuclear \& Mixed Waste Management Program

P.0. Box 47600

01ympia, WA 98504-7600

M. T. Gordon

M. Lerchan

Scott McKinney

R. Stanley

Library

P. 0. Box 1386

Richland, WA 99352

S. V. Moore

G. T. Tebb

Office Library

1

Washington State Department of Health

Radiation Protection Section

Industrial Park Building 5, LE-13

01 ympia, WA 98504

A. Conklin

1

General Accounting Office

P. 0. Box 321

Richland, WA 99352

C. R. Abraham 
WHC-EP-0182-74

Distribution - continued

Oregon State Department of Energy

625 Marion St. N.E.

Salem, OR 97310

Janet Franco

1

Oregon State Water Resources Department

Ground Water Hanford Studies

3850 Portl and Road

Salem, OR 97310

R. 0. Patt

1

Lawrence Livermore National Laboratory

Box 808, East Avenue

Livermore, CA 94550

B. C. Hudson

$L-221$

5

Oak Ridge National Laboratory

P. 0. Box 2009

Oak Ridge, TN 37831-6385

C. Forsberg

MS -6495

T. S. Kress

MS-8088

Bldg 9108

Chemical Technology Division

P. 0. Box 2008

Emory D. Collins

C. Phil McGinnis

Dr. Jack Watson

6

Los Alamos National Laboratory

P. 0. Box 1663

Los Alamos, NM 87545

Stephen Agnew

Group INC-14

Phyll is Baca

$C-346$

$\mathrm{J}-514$

T. Larson

C-915

Sylvia Lee

K-557

H. Sullivan

$N-6$

Dr. Reed Jensen

$\mathrm{J}-565$

Brookhaven National Laboratory Upton, NY 11973

K. K. Bandyopadhyay, B1dg 475-C

M. K. Kaisser, BIdg 475-C

P. D. Kalb, Bidg. 703

M. Reich, B1dg 475-C

J. R. Weeks, Bidg 197-C

Distr-3 
WHC-EP-0182-74

Distribution - continued

Brookhaven National Laboratory

1409 Jan Drive

Wilmington, DE 19803

Michael Streicher

Argonne National Laboratory

9700 South Cass Avenue

Argonne, IL 60439

Dr. Martin Steindler

Dr. George Vandergrift

Sandia National Laboratories

1515 Eubank, NE

P. O. Box 5800

Albuquerque, NM 87185

Scott Slezak, Division 6471

Leon D. Chapman, Program Manager

Industrial Waste Reduction Program

Dr. Margaret Chu, Organ \#6622

Elmer Klavetter, Mail Stop 0709

Norman E. Brown, Mail Stop 0728

Massachusetts Institute of Technology

77 Massachusetts Avenue

Cambridge, MA 02139

Mujid S. Kazimi

Professor and Head

Department of Nuclear Engineering

2

BDM International. Inc.

20030 Century Bivd, Suite 101

Germantown, MD 20874

P. Kiang

K. J. Mahoney

9

SAIC

20300 Century Blvd, Suite 200B

Germantown, MD 20874
J. Bunting
C. Herrington
J. R. Pearring
R. A. Wullaert

102 Windham Road

Oak Ridge, TN 37830

D. 0. Campbell 
WHC-EP-0182-74

\section{Distribution - continued}

1845 Terminal Drive, Suite 130

Richland, WA 99352

J. Mishima

555 Quince Orchard Road

Gaithersburg, MD 20878

Donald Oakley

Paul Szerszen

507 Knight Street, Suite B

Richl and, WA 99352

Pete Lowry

1

Harvard University

295 Upland Avenue

Newton Highlands, MA 02161

Melvin First

1

Syracuse University

334 Hinds $\mathrm{Hall}$

Chemical Engineering \& Materials Science Department

Syracuse, NY 13244

Dr. Larry Tavlarides

1

University of Washington

Chemical Engineering Department

Benson Hall, BF-10

Seattle, WA 98195

Professor Gene Woodruff

1

Washington State University

Department of Mechanical \& Materials Engineering

Pulliman, WA 99164-2920

Dr. Steve Antolovich, Chairman

1 Confederated Tribes, Umatilla Indian Reservation

P. 0. Box 638

Pendleton, OR 97801

Rick George 
WHC-EP-0182-74

\section{Distribution - continued}

4

Nest Valley Nuclear Services Co.

P. 0. Box 191

West Valley, NY 14171

K. K. Gupta

MS-49

S. Ketola

MS-191

Ram Shukla

Don Stroud

K \& Engineering \& Consulting Corporation

Suite 500

2001 L St. NW

Washington, D. C. 20036

Ryan Gill

5

Defense Nuclear Facil ities Safety Board

625 Indiana Ave, N. W., Suite 700

Washington, D. C. 20004

Ralph Arcaro

Lester Clemons

Suite 700

Steven Stokes

Richard Tontodonato

Dermot Winters

Westinghouse Idaho Nuclear Corporation

P. 0. Box 4000

Idaho Falls, ID 83404-4000

B. Griebenow

MS-5104

A. P. Hoskins

MS -5217

Dr. Terry Todd

1

C. Abrams

1987 Virginia Drive

Idaho Falls, ID 83404

1

Fred N. Carlson

6965 North 5 th West

Idaho Falls, ID 83401

1

Dr. A. Veletsos

Department of Civil Engineering

Rice University

P. O. Box 1892

Houston, TX 77252

2

Westinghouse Electric Corporation

1801 K Street NW, 8th floor

Washington DC, 20006

Kevin Billings 
WHC-EP-0182-74

Distribution - continued

6 Gateway Center

Pittsburgh, PA 15222

Steve Green, Manager

Westinghouse Environmental Affairs

Westinghouse Materials Company of Ohio

P. 0. Box 398704

Cincinnati $\mathrm{OH}$ 45239-8704

David L. Jacoboski

Senior Engineer, Technology Demonstration

3

Westinghouse Savannah River Company

P. 0. Box 616

Aiken, SC 29802

J. Marra, 703-H

F. G. McNatt, 704-8A

Dr. Major Thompson

1991 S. Centennial Ave.

Aiken, SC 29803

L. A. Wooten

Institute for Energy and Environmental Reseach

6935 Laurel Avenue

Takoma Park, MD 20912

Dr. Arjun Makhijani, President

1

SPAR Aerospace Ltd.

20 Avon Meadow Lane, Suite 220

Avon, CT 06001

Peter W. Kruse

Advanced Technology Systems Division

1

Redzone Robotics. Inc.

2425 Liberty Ave

Pittsburgh, PA 15222-4639

David W. White

National Research Council. National Academy of Sciences 2101 Constitution Ave., N. W.

Washington D. C. 20418

Robert S. Andrews, Senior Staff Officer

Board on Radioactive Waste Management 
HHC-EP-0182-74

\section{Distribution - continued}

\section{Converse Consultants}

$18 \mathrm{~W}$. Mercer Street, Suite 300

Seattle, WA 98119

David Stanley

1

Brown \& Caldwell

100 W. Harrison

Seattle, WA 98119

Hal Cooper

Benton County Department of Emergency Management

P.0. Box 6144

Kennewick, WA 99336

Gary Pira

1

BOVAY Northwest Inc.

660 Swift, Suite D

Richland, WA 99352

T. J. McLaughl in

1

Schilling Development. Inc.

1632 DaVinci Court

Davis, CA 95616

Wes Gerriets

1

T. S. Elleman

North Carolina State University

Department of Nuclear Energy

P. 0. Box 7909

Raleigh, NC 27606

1

Mike Lingle

Stone \& Webster

7677 E. Berry Ave

Englewood, CA 80111

1

Bryant Mather

Corps of Engineers

WESSV-Z

$3909 \mathrm{Hall}$ s Ferry Rd

Vicksburg, MS 39180-6199

1

Paul Shewmon

Prof. Metallurgical Engineer

Ohio State University

2477 Lytham Road

Columbus, $\mathrm{OH} 43220$ 
WHC-EP-0182-74

Distribution - continued

Waste Management External Advisory Committee Members

Dr. Frank L. Parker

Professor of Environmental and Water Resources Engineering Vanderbilt University

P. 0. Box 1596, Station B

Nashville, TN 37235

Dr. Bruce R. Kowalski

Professor of Chemistry, Co-director of Center for Process

Analytical Chemistry

University of Washington

Chemistry Department, Bldg 10

Seattle, WA 98195

Dr. Greg R. Choppin

Professor of Chemistry

Florida State University

Department of Chemistry, B-164

Tallahassee, FL 32306

Or. Chester Grelecki

President, Chief Scientist

Hazards Research Corporation

200 Valley Road, Suite 301

Mt. Arlington, NJ 07856

Or. Alfred Schneider

Professor Meritus of Nuclear Engineering

Georgia Institute of Technology

5005 Hidden Branches Dr.

Dunwoody, GA 30338

Dr. Gary Powers

President

Design Science, Inc.

163 Witherow Road

Sewickley, PA 15143

Joseph S. Byrd

University of South Carolina

Department of Electrical and Computer Engineering

Swearingen Engineering Center

Columbia, SC 29208

William R. Prindle

1556 Crestline Drive

Santa Barbara, CA 93105 
WHC-EP-0182-74

Distribution - continued

3

Ames Laboratory

7 Spedding $\mathrm{Hall}$

Iowa State University

Ames, IA 50011

Bill Haas

R. B. Thompson

Dr. Paul Wang

1

RKK Ltd.

16404 Smokey Pt. Blvd, Suite 303

Arlington, WA 98223

Chris Reno

1

MACTEC

8320 Centerbrook Place

Alexandria, VA 22308

Stan Blacker

1

Engineering-Science. Inc.

1955 Jadwin Ave, Suite 470

Richland, WA 99352

Matt Sakach

1

EBASCO Services, Inc.

1201 Jadwin Avenue, Suite 202

Richland, WA 99352-3429

F. J. Young

1

ENSEARCH Environmental

1981 Snyder Rd, Suite 3

Richland, WA 99352

Glen Cox

1

MTL Systems. Inc.

3481 Dayton-Xenia Road

Dayton, OH 45431-0299

E. McDaniel

1

Nuclear Consulting Services. Inc. 7000 Huntley Road

P. O. Box 29151

Columbus, $\mathrm{OH} 43229$

Dr. J. Louis Kovach 
WHC-EP-0182-74

\section{Distribution - continued}

1

Battelle Laboratories

505 King Avenue

Columbus, $\mathrm{OH}$ 43201-2693

Rob Taylor Jr., P.E.

Rm 13-6-016

Portland General Electric Co.

121 S. W. Salmon St.

Portland, OR 97204-2991

Wayne Le1, 3WTCBRO5

3

RUST Geotech Inc.

P. 0. Box 14000

Grand Junction, CO 81502-5504

Brian Mathis

Michael C. Butherus

John R. Duray

1

ICF Kaiser Engineers

1800 Harrison St.

Oakl and, CA 94612-3430

Chris G. Whipple, Ph.D

1

Neptune \& Company

1505 15th St., Suite B

Los Alamos, NM 87544

Randy Ryti

1

John Hopkins University

1714 Eutaw Place

Baltimore, MD 21217

Morton Corn

1

Roberts Engineering Services, INC.

1980 East Ocean Boulevard

Stuart, FL 34916

Dr. Paul Roberts

Columbia River United Technical Consultant

P. 0. Box 912

Bingen, WA 98605

Gregory de Bruler 
WHC-EP-0182-74

Distribution - continued

Government Accountability Project

West Coast Office

1402 Third Avenue, Suite 1215

Seattle, WA 98101

Thomas E. Carpenter, Director

1

Heart of America Northwest

1305 Fourth Avenue

Cobb Building Suite 208

Seattle, WA 98101

Gerald M. Pollet, Executive Director

1

Dr. Viorica Lopez-Avila

Midwest Research Institute

625-B Clyde Ave

Mountain View, CA 94043

3

Los Alamos Technical Associates

8633 W. Gage Blvd.

Kennewick, WA 99336

D. S. Delorenzo

Karen Todd

750 Swift, Suite 12

Richland, WA 99352

Janet Jones

2

RAPIC

Bidg K-1210

P. O. Box 2003

Oak Ridge, TN 37831-7256

Lola Estes

MS $72-56$

\section{OFFSITE - FOREIGN}

1

British Nuclear Fuels Ltd

Risley Warrington

Cheshire WA3 6AS

United Kingdom

Howard A. Edwards

1 Ricardo Hitec Ltd

Club street Works, Bamber Bridge Preston, PR5 6FN

United Kingdom

P. K. J. Smith 
WHC-EP-0182-74

Distribution - continued

1

Telerobot

Consorzio Telerobot

Via Hermada 6

16154 Genova, Italy

Bruno Sessarego

4

SGN

1 , rue des Herons, Montigny-le-Bretonneux

78182 Saint-Quentin-en-Yvelines Cedex,

France

Serge Merlin

Gilles Clement

Bernard Tousant

Eric Tchemitcheff

i

CEA - Saclay

DCC/DIR

$B a ' t 121$

91190 GIF/Yvette Cedex

France

G. Baudin

1

SDMS-Chaudronnerie Blanche

B.P. 4-F-38160 Saint-Romans

France

Phillipe Monneau

1

GEA-Cadarache

SGDC/STDE

Boite Pastale 313

13115 Saint Paul Les Durance

France

Magali Ranchoux

1

Hans Wal ischmiller GmbH

D-7778 Markdorf/Bodensee

Germany

Wolfgang Walischmiller

\section{ONSITE}

BNFL INC.

Dr. Mike McKeon G3-02 
WHC-EP-0182-74

Distribution - continued

Stone \& Webster Engineering Co.

E. L. Richards

R2-83

4

MACTEC
S. D. Barry
$57-73$
R. Coppin
R3-77
J. Janus
G6-18
L. Soler
A4-35

\section{EBASCO}
D. J. Leigh-Manuell
G3-02

Yakima Indian Nation

John Brodeur

G1-02

26

U. S. Department of Energy-Richland Operations Office

G. E. Bishop

$57-54$

K. W. Bracken

R3-73

S. T. Burnum

R3-74

R. C. Cullison

A5-55

J. J. Davis

R3-74

L. Erickson

S7-53

R. E. Gerton

R3-72

M. Glasper

R3-72

W. F. Hendrickson

S7-54

R. L. Higgins

R3-72

R. D. Hildebrand

A5-55

A. Hon

R3-72

P. E. LaMont

S7-53

C. Z. Morgan

R3-72

T. Noble

R3-72

M. S. Peck

T4-07

L. E. Petersen

R3-72

G. W. Rosenwald

R3-72

C. 0 . Ruud

S7-54

G. H. Sanders

R3-74

T. R. Sheridan

R3-73

A. B. Sidpara

R3-73

A. D. Toth

R3-72

D. L. Vieth

R3-73

D. J. Williams

S7-54

J. K. Yerxa

A5-15

Reading Room

A $1-65$

Pacific Northwest Laboratories

D. N. Anderson

K7-34

W. J. Apley

$\mathrm{K} 1-73$

D. B. Baird

K $7-34$ 


\section{Distribution - continued}

R. M. Bean

P8-08

D. W. Bennett

$\mathrm{K} 5-17$

P. R. Bredt

P7 -25

J. C. Brown

$K 7-22$

S. A. Bryan

P7 -25

L. L. Burger

$P 7-25$

J. B. Colson

K5-10

J. M. Creer

G6-01

P. G. Eller

B1-40

J. F. Fletcher

K7 -97

E. A. Flores

K3-08

L. K. Holton Jr.

P7 -43

V. L. Hunter

K7 -94

P. R. Hrma

P8-37

J. Janata

$\mathrm{K} 2-12$

B. M. Johnson

$\mathrm{K} 1-78$

E. 0 . Jones

P8-38

D. E. Larson

G3-02

S. G. Mckinley

P7-22

B. D. McVeety

K6-63

I. C. McVeety

K7-22

P. J. Mellinger

P7 -22

B. E. Opitz

K6-79

M. D. Patridge

K7-94

M. S. Peffers

K7-94

R. K. Quinn

$\mathrm{K} 1-73$

W. G. Richmond

P7-41

J. M. Robbins

P7-22

R. D. Scheele

P7 -25

E. A. Schmieman

B1 -40

R. C. Schrotke

P7 -18

P. A. Scott

P7-19

D. A. Seaver

$\mathrm{K} 1-73$

J. C. Spanner

J. L. Straalsund

K2-05

K. L. Steinmaus

$\mathrm{K} 1-79$

K6-84

J. L. Swanson

K. M. Tominey

J. D. Vienna

P7-25

K7-97

P8-44

R. S. Wegeng

P. D. Whitney

K7-97

K7-34

T. W. Wood

K6-47 Sciences Richland/ICF Kaiser Hanford Company

L. K. Ahrens

H6-07

A. T. Alstad

Rl-49

W. T. Alumkal

R2-52

R. P. Anantatmula

R2-11

J. D. Anderson

N3-11

J. N. Appel

I. J. Aust in

S4-58

H. Babad

T4-01

S7 -30 


\begin{tabular}{|c|c|}
\hline $\begin{array}{l}\text { J. J. Badden } \\
\text { J. K. Bajwa } \\
\text { R. A. Ballou } \\
\text { T. J. Bander } \\
\text { A. W. Barthoff } \\
\text { A. D. Bates } \\
\text { L. L. Barry } \\
\text { G. D. Bazinet } \\
\text { D. B. Bechtold } \\
\text { D. L. Becker } \\
\text { L. Bedford } \\
\text { K. E. Bell } \\
\text { R. V. Berg } \\
\text { M. V. Berriochoa } \\
\text { P. K. Bhatia } \\
\text { K. R. Birney } \\
\text { D. L. Bjorklund } \\
\text { J. E. Bjorklund } \\
\text { R. J. Blanchard } \\
\text { J. W. Bloom } \\
\text { D. C. Board } \\
\text { K. D. Boomer } \\
\text { G. L. Borsheim } \\
\text { V. C. Boyles } \\
\text { D. A. Bragg } \\
\text { D. R. Bratzel } \\
\text { W. R. Brooksher } \\
\text { T. M. Brown } \\
\text { R. G. Brown } \\
\text { J. H. Bussel1 } \\
\text { J. A. Caggiano Jr } \\
\text { T. M. Cameron } \\
\text { J. W. Carey } \\
\text { K. G. Carothers } \\
\text { B. C. Carpenter } \\
\text { R. J. Cash } \\
\text { T. Chiao } \\
\text { G. Christensen } \\
\text { K. L. Chubb } \\
\text { R. H. Clements } \\
\text { G. J. Coleman } \\
\text { J. C. Conner } \\
\text { R. B. Conrad } \\
\text { W. L. Cowley } \\
\text { D. W. Craig } \\
\text { N. R. Croskrey } \\
\text { G. M. Crummel } \\
\text { J. M. Cruse } \\
\text { D. S. Cunningham } \\
\text { J. E. Daniels } \\
\text { J. S. Davis } \\
\text { S. J. Dechter } \\
\text { C. DeFigh-Price } \\
\text { J. L. Deichman }\end{array}$ & 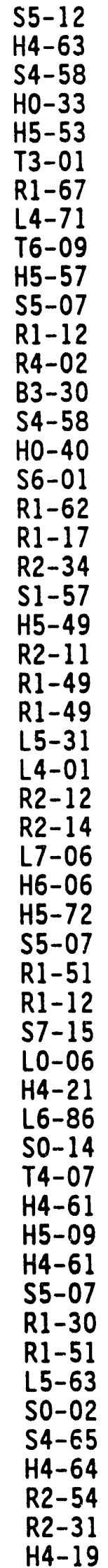 \\
\hline
\end{tabular}




\section{Distribution - continued}

T. A. Demitruk

H5-36

C. J. Denson

L4-95

S. E. Dieterle

S2-20

L. F. Dougherty

H4 -62

J. G. Douglas

L5-55

G. L. Dunford

RI-51

R. F. Eggers

S4-58

D. R. Ellingson

H5-37

F. W. Ellis

D. B. Engelman

B4-52

R. W. Ewert

S. D. Estey

W. G. Farley

J. E. Fasso Jr.

R2-18

RI-19

R2-11

H4-62

G. W. Faulk

S3-10

K. 0 . Fein

T3-28

L. A. Fort

$\mathrm{H} 4-63$

K. D. Fowler

S4-57

S. B. Fowler

R2-11

C. R. Fox

LO-24

G. L. Fox, Jr.

S4-60

H. P. Fox

L5-01

G. T. Frater

R2-88

E. A. Fredenburg

S7 -30

J. R. Freeman-Pollard

S4-55

R. T. French

L. A. Gadd is

H6-03

E6-61

K. A. Gasper

G7-57

G. J. Gauck

G3-20

C. J. Geier

RI-51

P. W. Gibbons

K. D. Gibson

R. L. Gilchrist

R2-50

S4-58

H4-61

L5-63

D. A. Gilles

S2-14

S. D. Godfrey

Rl-51

D. E. Good

S3-97

A. Greenberg

P. R. Golberg

P. Greenbaum

S2-66

B4-08

S5-20

H4-62

J. M. Grigsby

R1-51

R. D. Gustavson

H4-61

R. L. Guthrie

R1-30

J. H. Haberman

T4-09

K. D. Haggerty

E6-25

L. E. Hall

V. W. Hall

C. S. Haller

H4-19

G6-04

S7-03

D. W. Hamilton

S4-53

K. L. Hampsten

B. M. Hanion (25)

J. M. Hanson

R1-80

R2-85

M. S. Harrington

B4 -53

J. P. Harris III

S4-55

G. A. Harvey

B4-52 


\section{Distribution - continued}

F. J. Heard

$\mathrm{HO}-34$

D. A. Healey

S7-04

J. M. Henderson

S4-55

D. W. Hendrickson

L5-31

M. C. Higginson

A4-25

K. S. Hoeft

GI-61

M. J. Holm

$\mathrm{R} 1-80$

C. S. Homi

R2-12

G. P. Hopkins

$\mathrm{N} 2-40$

J. D. Hopkins

R2-11

B. K. Horsager

B5-24

J. H. Huber

J. L. Huckaby

RI-49

J. V. Hurley

S7-15

J. J. Huston

R4-02

J. E. Irvin

S1-54

M. N. Islam

S7-83

R3-08

M. T. Jansky

H6-26

T. D. Jarecki

S7-12

P. Jennings

SO- 14

D. W. Jeppson

L5-31

R. D. Jensen

B1-58

G. D. Johnson

S7 -15

L. J. Julyk

H5-56

R. A. Karnesky

HO-39

D. L. Kelly

S5-20

R. A. Kirkbride

S4-58

P. F. Kison

S2-45

C. A. Kuhlman

B3-30

N. W. Kirch

R2-11

D. B. Klos

SO-14

A. G. Krasopoulos

A5-55

M. Kummerer

M. J. Kupfer

H4 -62

E. C. Ladd

H5- 49

Rl-19

D. R. Lance

J. L. Lee

SO-09

S7-82

J. M. Light

B4-08

D. C. Lini

H3-56

B. H. Lueck Jr.

R3-12

P. J. Mackey

B3-15

G. T. Maclean

S4-58

R. M. Marusich

H4 -60

V. D. Maupin

T. B. McCall

$\mathrm{N} 1-73$

HO-33

K. S. McCullough

H4 -70

M. H. McGrath

J. P. Menard

S4-58

R2-40

S4-55

W. C. Miller

H4-63

N. J. Milliken

H5-68

W. J. Millsap

G. J. Miskho

R2-50

J. R. Mobley

H5 -49

T. Moleff

RI-30 


\section{Distribution - continued}

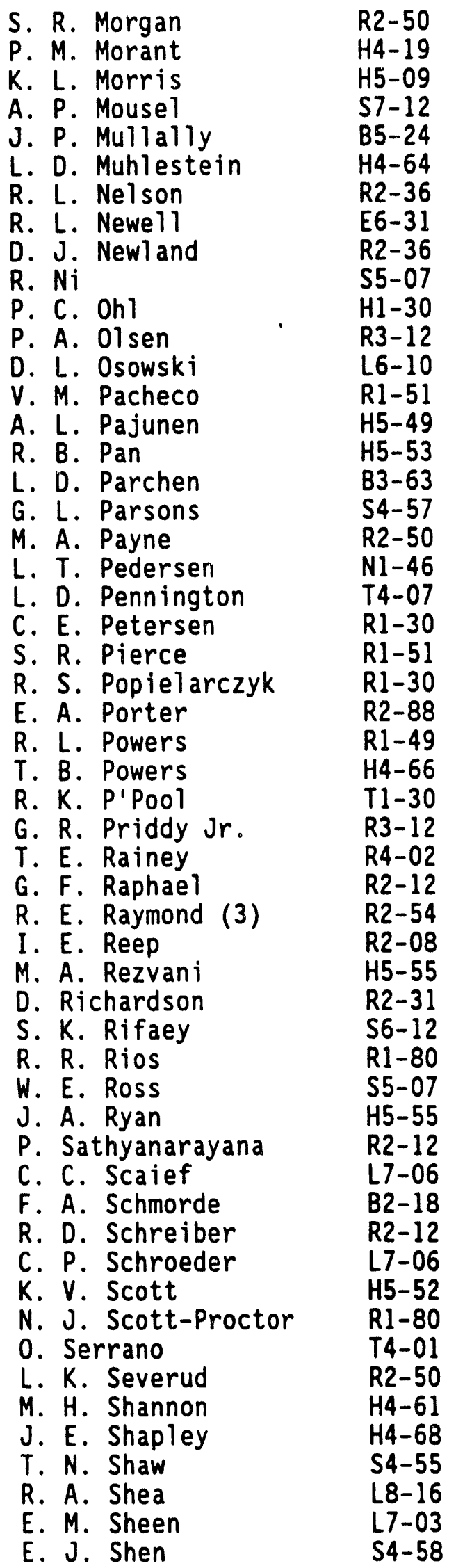




\section{Distribution - continued}

P. K. Shen

D. J. Sherwood

A. T. Shook

E. R. Siciliano

R. B. Simmons

K. P. Slape

C. M. Smith

S. M. Stah1

E. G. Stephan

B. E. Stapley

R. R. Stickney

J. N. Strode

D. G. Sutherl and

J. P. Summerhays

M. J. Sutey

S. L. Swaney

L. M. Swanson

C. L. Thomas

J. F. Thompson

J. D. Thomson

S. R. Tifft

J. A. Tilden (2)

H. Toffer

T. T. Tran

J. W. Tritz

D. P. Trott

D. A. Turner

L. A. Tusler

C. J. Udell

B. D. Valenzuela

R. E. Van der Cook

R. J. Van Vleet

A. 0 . Vance

N. J. Vermeulen

D. T. Vladimiroff

F. R. Vollert

J. A. Voogd

G. R. Walker

O. S. Wang

D. L. Wegener

R. K. Welty (3)

G. T. Wells

K. A. White

$R$. Whitman

R. S. Whittman

J. H. Wicks

D. D. Wiggins

L. S. Williams

C. R. Wilson

G. R. Wilson

M. E. Witherspoon

D. D. Wodrich

K. L. Woodard

R. D. Wojtasek
HO-39

R2 -78

S2-01

HO-39

$\mathrm{R} 2-12$

SO-11

H6-30

H4-61

A3-74

R4-02

R4-02

R2-11

L4-72

R2-88

T4-08

T4-08

H5-49

H4- 19

H5-68

R1-30

H6-26

L6-12

HO-38

L7. -04

B1-32

R2-86

R2-78

R2-11

L6-12

R2-12

H5-27

H4-63

H5-33

R1-80

G7-01

H5-09

R4-03

B2-16

R2-78

R1-62

R1-80

H6-26

R1-51

H5-49

B1-59

T4-07

R1-49

S4-53

H5-52

R2-31

E6-61

57-84

R4-02

H6-27 
HHC-EP-0182-74

\section{Distribution - continued}

G. D. Wright

G6-04

W. R. Wrzesinski

R3-74

P. A. Young

R2-14

F. A. Zak

R3-01

B. D. Zimmerman

LO-06

SDPC Docket File (2) H5-36

272-AW Shift office S5-04

Central Files L8-04

Tank Farms Info Center Rl-20

Information Release

Administration

R1-05

Env Data Mgmt Center H4-22

RL/TWRS Library R3-72 

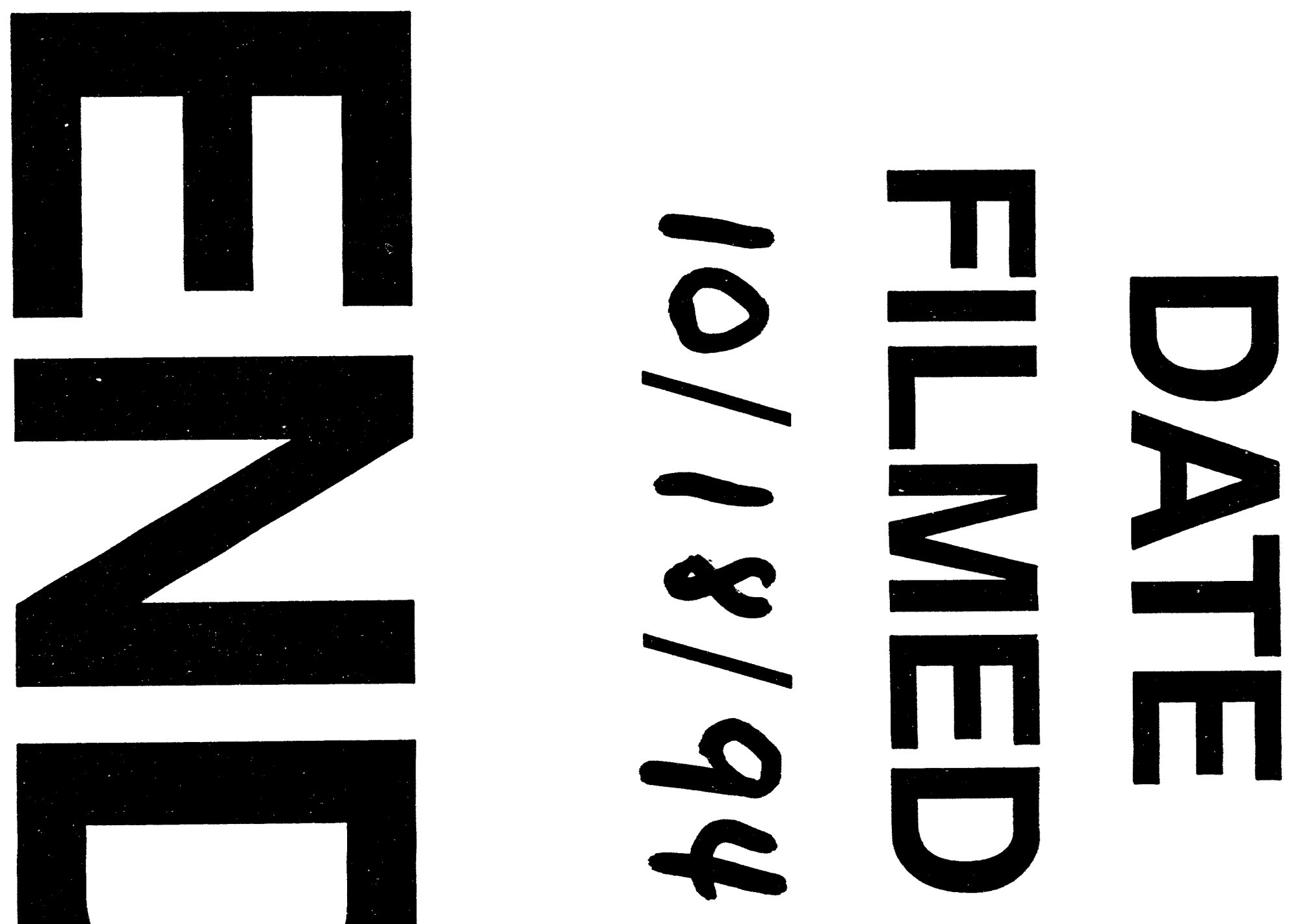


$$
\text { nemen }
$$

